\title{
Boron accumulation in brain tumor cells through Boc-protected tryptophan as a carrier for boron neutron capture therapy
}

Chun-Ming Chio, ${ }^{\mathrm{a},+}$ Ying-Cheng Huang, ${ }^{\mathrm{b},+}$ You-Cheng Chou, ${ }^{\mathrm{a}}$ Fu-Chun Hsu ${ }^{\mathrm{a}}$, Yen-Buo Lai ${ }^{\mathrm{a}}$ and ChungShan $\mathrm{Yu}^{\mathrm{a}, \mathrm{c}^{*}}$

${ }^{\text {a} D e p a r t m e n t ~ o f ~ B i o m e d i c a l ~ E n g i n e e r i n g ~ a n d ~ E n v i r o n m e n t a l ~ S c i e n c e s, ~ N a t i o n a l ~ T s i n g h u a ~ U n i v e r s i t y, ~ H s i n c h u ~ 300, ~ T a i w a n ~}$

${ }^{\text {b} D e p a r t m e n t ~ o f ~ N e u r o s u r g e r y, ~ C h a n g-G u n g ~ M e m o r i a l ~ H o s p i t a l ~ a t ~ L i n k o u, ~ C h a n g ~ G u n g ~ U n i v e r s i t y, ~ T a i w a n . ~}$

'Institute of Nuclear Engineering and Science, National Tsing-Hua University, Hsinchu, 300, Taiwan.

$\dagger$ equal contribution

Contents

$\begin{array}{ll}\text { Experimental section } & \text { S1-S3 } \\ \text { Synthesis of compound 1-18 } & \text { S3-S25 } \\ \text { Cell survival assay } & \text { S25 } \\ \text { Cell accumulation assay } & \text { S26 } \\ \text { Cell eradication assay after neutron irradiation } & \text { S26 } \\ \text { Western blot assay } & \text { S27 } \\ \text { Copies of NMR Spectra, HRMS Spectra and HPLC Purity Analysis } & \text { S28 } \\ \text { Cytotoxicities of boronopincaol tryptophan derivatives } & \text { S97 } \\ \text { References } & \text { S98 }\end{array}$

1. Experimental section

\subsection{General Information}

All reagents and solvents were purchased from Sigma-Aldrich (St. Louis, MO, USA), Fluka (St. Louis, MO, USA), Acros (Geal, Belgium), Alfa (Binfield, Berkshire, UK), Malingkrodt (Phillipsburg, NJ, USA), or Tedia (Farfield, OH, USA). Preparation of all compounds was routinely conducted in dried glassware under a positive pressure of nitrogen at room temperature unless otherwise noted. $\mathrm{CH}_{2} \mathrm{Cl}_{2}$, toluene, $\mathrm{CH}_{3} \mathrm{CN}$, and 
pyridine were dried over $\mathrm{CaH}_{2}$ and $\mathrm{CH}_{3} \mathrm{OH}$ was dried over $\mathrm{Mg}$ and distilled prior to their use in reactions. Solvents (e.g., DMF and $\left.\mathrm{N}(i-\mathrm{Pr})_{2} \mathrm{Et}\right)$ were distilled under reduced pressure. All the reagents and solvents were of reagent grade. Solvents for flash chromatography e.g. EtOAc, $n$-hexane and acetone were industrial grade and have been distilled before use. Dimethylaminopyridine (DMAP) was purified by recrystallizing the reagent from a combination of EtOAc and $n$-hexane before use. The eluents for flash chromatography (e.g., EtOAc, acetone, and $n$-hexane) were of industrial grade and were distilled prior to use; $\mathrm{CH}_{3} \mathrm{OH}$ and $\mathrm{CHCl}_{3}$ were of reagent grade and used without further purification. NMR spectroscopy including ${ }^{1} \mathrm{H}-\mathrm{NMR}(500 \mathrm{MHz}),{ }^{13} \mathrm{C}-$ NMR (125 MHz, DEPT-135) and ${ }^{19} \mathrm{~F}-\mathrm{NMR}$ (470 or $564 \mathrm{MHz}$ ) was performed on a Unity Inova $500 \mathrm{MHz}$ instrument (Varian, USA). Deuterated-solvents employed for NMR spectroscopy including $\mathrm{CD}_{3} \mathrm{OD}, \mathrm{CDCl}_{3}$, $\mathrm{C}_{6} \mathrm{D}_{6}$, and DMSO- $d_{6}$ were purchased from Aldrich (St. Louis, MO, USA). Low-Resolution Mass Spectrometry (LRMS) was performed on an ESI-MS spectrometer using a Varian 901-MS Liquid Chromatography Tandem Mass Q-TOF Spectrometer at the Department of Chemistry of National Tsing-Hua University (NTHU) or the Department of Applied Chemistry of National Chio-Tung University (NCTU). High-Resolution Mass Spectrometry (HRMS) was performed using a Varian HPLC (prostar series ESI/APCI) system coupled with a Varian 901-MS (FT-ICR Mass) mass detector and a triple quadrapole instrument. Thin layer chromatography (TLC) was performed with TLC silica gel $60 \mathrm{~F}_{254}$ pre-coated plates (Machery-Nagel, Dueren, Germany) to monitor the starting materials and products with visualization under UV light (254 nm). Further confirmation was carried out by staining the TLC plates with ninhydrin or ceric ammonium molybdate under heating. Celite 545 was purchased from Macherey-Nagel Inc. (Dueren, Germany). Flash chromatography was performed using Silicycle 60 silica gel (70-230 mesh, Quebec city, Quebec, Canada). Melting points were measured with a MEL-TEMP instrument (Barnstead international, Dubuque, IOWA, USA) and were uncorrected.

Reversed phase HPLC system (Milford, MA, USA) consists of an Angilent quarternary 1200 pump. Normal phase HPLC comprises an Angilent isocratic 1100 pump. Both HPLC systems connect a UVIS detector $(254 \mathrm{~nm})$ and a column of CHIRALCEL® OD-RH 5 um 4.6×150 mm (reverse phase) or ZORBAX SIL column $(9.4 \mathrm{~mm} \times 250 \mathrm{~mm}, 5 \mu \mathrm{m})$. Normal phase HPLC uses a combination of EtOAc and $n$-hexane as the mobile phase with a flow rate of $3 \mathrm{~mL} / \mathrm{min}$. Reversed phase $\mathrm{HPLC}$ uses a combination of $\mathrm{H}_{2} \mathrm{O}$ and $\mathrm{EtOH}$ as the 
mobile phase with a variant flow rate ranging from 0.6 to $0.8 \mathrm{~mL} / \mathrm{min}$. A Rheodyne injector with a loop of 0.5 $\mathrm{mL}$ was employed for each system.

\subsection{Synthesis}

The preparation of most of the target compounds have been reported. The reaction conditions and the methods modified are described in the supporting information. The typical procedure for the preparation of boronopinacol tryptophan derivatives is described below.

\section{1,3-(2R,2S)(2-N,N)triBoc-5-bromotryptophan methyl ester 7}

1) Introduction of Methyl ester and one Boc group

The $d, l$ racemic mixture of 5-bromotryptophan was analyzed using reversed phase HPLC. Through a gradient mode of eluent combinations of $\mathrm{H}_{2} \mathrm{O} / \mathrm{EtOH}$ 100:0 (0-3 min), 90:10 (3-6min) and 80:20 (6 min ) and a flow rate of $0.8 \mathrm{~mL} / \mathrm{min}$ and $\mathrm{UV}$ absorption of $\lambda_{\max }$ at $254 \mathrm{~nm}$, two peaks at $t_{R 1}=8.17$ min and $t_{R 2}=$ $10.27 \mathrm{~min}$ were recorded in a ratio of 4:1. A round-bottomed two-neck flask (250 $\mathrm{mL})$ was charged with 5bromotryptophan $(3.5 \mathrm{~g}, 12.36 \mathrm{mmol})$ and $\mathrm{CH}_{3} \mathrm{OH}(45 \mathrm{~mL})$ under $\mathrm{N}_{2}$. After cooling with an ice bath, $\mathrm{SOCl}_{2}$ ( $5 \mathrm{~mL}, 70 \mathrm{mmol}, 6 \mathrm{eq}$ ) was added. The mixture was refluxed and the temperature was set at $130{ }^{\circ} \mathrm{C}$. TLC $\left(\mathrm{CH}_{3} \mathrm{OH} / \mathrm{CH}_{2} \mathrm{Cl}_{2} 6: 4\right)$ indicated consumption of the starting material $\left(\mathrm{R}_{f}=0.20\right)$ and formation of the ester protected intermediate $\left(\mathrm{R}_{f}=0.89\right)$. After $50 \mathrm{~min}$, the mixture was concentrated under reduced pressure. $\mathrm{CH}_{3} \mathrm{OH}(10 \mathrm{~mL})$ and $\mathrm{NEt}_{3}(30 \mathrm{~mL})$ was added for quenching. $\mathrm{CH}_{3} \mathrm{OH}(50 \mathrm{~mL})$ was added followed by concentration under reduced pressure. The residue was dissolved in $\mathrm{CH}_{3} \mathrm{OH}(30 \mathrm{~mL})$ followed by addition of $\mathrm{Et}_{3} \mathrm{~N}(24.12 \mathrm{~mL}, 0.17 \mathrm{~mol}, 14 \mathrm{eq})$ and $\mathrm{Boc}_{2} \mathrm{O}(4.30 \mathrm{~mL}, 18.7 \mathrm{mmol}, 1.5 \mathrm{eq})$, sequentially. While stirring under protection with $\mathrm{N}_{2}$, the solution turned pale yellow. TLC (acetone/n-hexane 4:6) indicated 
consumption of the ester intermediate $\left(\mathrm{R}_{f}=0.18\right)$ and formation of the monoBoc intermediate $\left(\mathrm{R}_{f}=0.75\right)$.

After $30 \mathrm{~min}$, the mixture was concentrated at $40{ }^{\circ} \mathrm{C}$ under reduced pressure. The residue was dissolved in EtOAc $(80 \mathrm{~mL})$ followed by washing with $1 \mathrm{~N} \mathrm{HCl}_{(\mathrm{aq})}(12 \mathrm{~mL})$ twice and $\mathrm{NaOH}(8 \mathrm{~mL})$ once. The organic layer was collected, dried over $\mathrm{MgSO}_{4}$ and filtered. The filtrate was concentrated under reduced pressure to give a white foam in a quantitative yield $(6.67 \mathrm{~g}, 16.80 \mathrm{mmol})$.

2) Introduction of two Boc groups

The above residue of monoBoc ester was dissolved in $\mathrm{CH}_{3} \mathrm{CN}(20 \mathrm{~mL})$ followed by evaporation with additional $\mathrm{CH}_{3} \mathrm{CN}(50 \mathrm{~mL})$. The mixture was protected under $\mathrm{N}_{2}$ and a mixture of DMAP $(1.50 \mathrm{~g}, 12.3 \mathrm{mmol}$, 1 eq) and $\mathrm{Boc}_{2} \mathrm{O}(8.50 \mathrm{~mL}, 37.0 \mathrm{mmol}, 3 \mathrm{eq})$ dissolved in $\mathrm{CH}_{3} \mathrm{CN}(10 \mathrm{~mL})$ was added. Followed by addition of the washings with $\mathrm{CH}_{3} \mathrm{CN}(10 \mathrm{~mL})$, the solution turned from clearly orange to turbid. TLC (EtOAc/nhexane 1:9) indicated consumption of the monoBoc ester $\left(\mathrm{R}_{f}=0.05\right)$ and formation of the tri-Boc product 7 $\left(\mathrm{R}_{f}=0.50\right)$. After $3 \mathrm{~h}$, an additional portion of DMAP $(0.55 \mathrm{~g}, 4.5 \mathrm{mmol}, 0.35 \mathrm{eq})$ and $\mathrm{Boc}_{2} \mathrm{O}(2.0 \mathrm{~mL}, 8.7$ mmol, 0.7 eq $)$ in $\mathrm{CH}_{3} \mathrm{CN}(10 \mathrm{~mL})$ was added. After $1 \mathrm{~h}$, the second portion of DMAP $(0.30 \mathrm{~g}, 2.5 \mathrm{mmol}, 0.2$ eq) and $\mathrm{Boc}_{2} \mathrm{O}(1.0 \mathrm{~mL}, 4.4 \mathrm{mmol}, 0.35 \mathrm{eq})$ in $\mathrm{CH}_{3} \mathrm{CN}(10 \mathrm{~mL})$ was added. After $0.5 \mathrm{~h}$, the reaction was terminated by distillation of the solvent. The residue was transferred to extraction funnel using EtOAc $(90 \mathrm{~mL})$ followed by washing with $1 \mathrm{~N} \mathrm{HCl}_{(\mathrm{aq})}\left(12 \mathrm{~mL}\right.$ ) twice and satd. $\mathrm{Na}_{2} \mathrm{CO}_{3}$ (aq., $10 \mathrm{~mL}$ ) once. The organic layer was collected, dried over $\mathrm{MgSO}_{4}$ and filtered through gravitational filtration. The filtrate was concentrated at $40{ }^{\circ} \mathrm{C}$ under reduced pressure to give a residue then this was followed by flash chromatography with eluents inder gradient mode of EtOAc/ $n$-hexane 1:19 $\rightarrow 1: 14 \rightarrow 1: 9$, a yellow viscous liquid 7 was obtained in 93\% yield (6.90 g, $11.55 \mathrm{mmol})$. 
${ }^{1} \mathrm{H}-\mathrm{NMR}\left(500 \mathrm{MHz}, \mathrm{CDCl}_{3}\right) \delta 1.34$ (s, 18H, Boc), 1.63 (s, 9H, Boc), 3.34 (dd, J = 15.0 Hz, J = 10.0 Hz, 1H, $\left.\mathrm{H}_{\text {Aliphatic }}, \mathrm{H}-1 \mathrm{a}^{\prime}\right), 3.46$ (dd, J = 15.0 Hz, J = 5.0 Hz, 1H, HAliphatic, H-1b'), 3.77 (s, 3H, COOCH $), 5.14$ (dd, J = $\left.10.0 \mathrm{~Hz}, \mathrm{~J}=5.0 \mathrm{~Hz}, 1 \mathrm{H}, \mathrm{H}_{\text {Aliphatic }}, \mathrm{H}-2^{\prime}\right), 7.26 \sim 7.39$ (m, 2H, $\left.\mathrm{H}_{\text {arom }}\right), 7.63$ (s, 1H, $\mathrm{H}_{\text {arom }}$ ), 7.98 (d, J = 5.0 Hz, 1H, $\left.\mathrm{H}_{\text {arom }}\right) .{ }^{13} \mathrm{C} \mathrm{NMR}\left(125 \mathrm{MHz}, \mathrm{CDCl}_{3}\right) \delta 25.44\left(\mathrm{CH}_{2}, \mathrm{C}-1^{\prime}\right), 27.73\left(\mathrm{CH}_{3}, \mathrm{C}\left(\underline{\mathrm{C}} \mathrm{H}_{3}\right)_{3}\right), 28.11\left(\mathrm{CH}_{3}, \mathrm{C}\left(\underline{\mathrm{CH}_{3}}\right)_{3}\right), 52.36$ $\left(\mathrm{CH}_{3}, \mathrm{CO}_{2} \underline{\mathrm{CH}}_{3}\right), 58.37\left(\mathrm{CH}, \mathrm{C}-2^{\prime}\right), 83.13\left(\mathrm{C}, \underline{\mathrm{C}}\left(\mathrm{CH}_{3}\right)_{3}\right), 83.92\left(\mathrm{C}, \underline{\mathrm{C}}\left(\mathrm{CH}_{3}\right)_{3}\right), 115.91(\mathrm{C}$, arom $), 115.96(\mathrm{C}$, arom), $116.63(\mathrm{CH}$, arom), $121.76(\mathrm{CH}$, arom $), 125.45(\mathrm{CH}$, arom $), 127.13(\mathrm{CH}$, arom $), 132.34(\mathrm{C}$, arom), $134.19\left(\mathrm{C}\right.$, arom), $149.20\left(\mathrm{C}, \underline{\mathrm{CO}_{2}} \mathrm{C}\left(\mathrm{CH}_{3}\right)_{3}\right), 151.83\left(\mathrm{C}, \underline{\mathrm{CO}}_{2} \mathrm{C}\left(\mathrm{CH}_{3}\right)_{3}\right), 170.63\left(\mathrm{C}, \underline{\mathrm{CO}}_{2} \mathrm{CH}_{3}\right)$. High-resolution MS (HRMS): ESI-Q-TOF MS (m/z) calcd. for $\mathrm{C}_{27} \mathrm{H}_{37} \mathrm{BrN}_{2} \mathrm{O}_{8},[\mathrm{M}+\mathrm{Na}]^{+}=619.1631(100.0 \%), 620.1665$ (29.2\%), 621.1611 (97.3\%); found: $[\mathrm{M}+\mathrm{Na}]^{+}=619.1593(96.0 \%), 620.1623(26.5 \%), 621.1577(100.0 \%) . \delta$ $=-6.1 \mathrm{ppm}$.

\section{1,3-(2R,2S)(2-N,N)triBoc-5-boronopiacoltryptophan methyl ester TB-5-BT 1}

The reaction condition followed the procedure that described for preparation of TB-6-BT 5. KOAc (1.3 $\mathrm{g}), \mathrm{B}_{2} \mathrm{Pin}_{2}(1.73 \mathrm{~g}), \mathrm{Pd}(\mathrm{dppf}) \mathrm{Cl}_{2}(450 \mathrm{mg})$ and starting material 7 (1.95 g, $\left.3.26 \mathrm{mmol}\right)$ were used. The solvent DMSO used for transferring was $35 \mathrm{~mL}$. Reaction temperature was kept at $90{ }^{\circ} \mathrm{C}$. The reaction was terminated at 60 min postreaction. TLC (EtOAc/n-hexane 2:8) indicated consumption of the starting material $7\left(\mathrm{R}_{f}=0.64\right)$ and formation of the product TB-5-BT $1\left(\mathrm{R}_{f}=0.60\right)$. The black crude mixture was purified using flash chromatography with a gradient mode of EtOAc/n-hexane $1: 19 \rightarrow 1: 9$ to give the pleasant white crystal product in $95 \%$ yield $(2.10 \mathrm{~g}, 3.12 \mathrm{mmol})$. Analytical data matched literature values. ${ }^{1}$

${ }^{1} \mathrm{H}-\mathrm{NMR}\left(500 \mathrm{MHz}, \mathrm{CDCl}_{3}\right) \delta 1.30(\mathrm{~s}, 18 \mathrm{H}, \mathrm{Boc}), 1.33$ (s, 12H, pinacol), 1.62 (s, 9H, Boc), 3.36 (dd, J = 15.0, J = 10.5 Hz, 1H, H-1a'), 3.52 (dd, J = 15.0, J = 4.5 Hz, 1H, H-1b'), $3.76\left(\mathrm{~s}, 3 \mathrm{H}, \mathrm{COOCH}_{3}\right), 5.21(\mathrm{dd}, \mathrm{J}$ 
$\left.=10.5, \mathrm{~J}=4.5 \mathrm{~Hz}, 1 \mathrm{H}, \mathrm{H}-2^{\prime}\right), 7.34\left(\mathrm{~s}, 1 \mathrm{H}, \mathrm{H}_{\text {arom }}, \mathrm{H}-2\right), 7.72\left(\mathrm{~d}, \mathrm{~J}=8.0 \mathrm{~Hz}, 1 \mathrm{H}, \mathrm{H}_{\text {arom }}, \mathrm{H}-7\right), 7.97$ (s, 1H, $\left.\mathrm{H}_{\text {arom }} \mathrm{H}-4\right), 8.08$ (d, J = 7.5 Hz, 1H, $\left.\mathrm{H}_{\text {arom }} \mathrm{H}-6\right) .{ }^{13} \mathrm{C}-\mathrm{NMR}\left(125 \mathrm{MHz}, \mathrm{CDCl}_{3}\right) \delta 24.86\left(\mathrm{CH}_{3}, \mathrm{Bpin}\right), 24.90$ $\left(\mathrm{CH}_{3}, \mathrm{Bpin}\right), 25.42\left(\mathrm{CH}_{2}, \mathrm{C}-1^{\prime}\right), 27.74\left(\mathrm{CH}_{3}, \mathrm{Boc}\right), 28.16\left(\mathrm{CH}_{3}, \mathrm{Boc}\right), 52.29\left(\mathrm{CH}_{3}, \mathrm{CO}_{2} \mathrm{CH}_{3}\right), 58.16(\mathrm{CH}, \mathrm{C}-$ 2'), $82.92\left(\mathrm{C}, \mathrm{C}\left(\mathrm{CH}_{3}\right)_{3}\right), 83.51\left(\mathrm{C}, \mathrm{C}\left(\mathrm{CH}_{3}\right)_{3}\right), 83.63\left(\mathrm{C}, \mathrm{C}\left(\mathrm{CH}_{3}\right)_{3}\right), 114.50(\mathrm{CH}$, arom), 116.72 (C, arom), 122.54 (bs, C, arom-boronopin), $124.38(\mathrm{CH}$, arom), $126.02(\mathrm{CH}$, arom $), 130.05(\mathrm{C}$, arom), $130.86(\mathrm{CH}$, arom), $137.61\left(\mathrm{C}\right.$, arom), $149.47\left(\mathrm{C}, \underline{\mathrm{CO}_{2}} \mathrm{C}\left(\mathrm{CH}_{3}\right)_{3}\right), 151.64\left(\mathrm{C}, \underline{\mathrm{CO}_{2}} \mathrm{C}\left(\mathrm{CH}_{3}\right)_{3}\right), 170.87\left(\mathrm{C}, \underline{\mathrm{CO}}_{2} \mathrm{CH}_{3}\right)$. Analysis for $\mathrm{C}_{33} \mathrm{H}_{49} \mathrm{BN}_{2} \mathrm{O}_{10}$, calcd. for $[\mathrm{M}+\mathrm{Na}]^{+}(\mathrm{m} / \mathrm{z})=667.3378(100.0 \%), 668.3412(35.7 \%), 666.3414(24.8 \%)$; ESI-Q-TOF HR-ESI-MS found: $[\mathrm{M}+\mathrm{Na}]^{+}=667.3386(100.0 \%), 668.3417(37.0 \%), 666.3415(20.9 \%) . \delta=$ $1.2 \mathrm{ppm}$.

3-(2R,2S)-5-boronopiacoltryptophan methyl ester 5-BT 4

To a two-necked round-bottomed flask was charged TB-5-BT 1 (99.5 mg, $0.15 \mathrm{mmol}$, leq) and $\mathrm{CHCl}_{3}$ $(4 \mathrm{~mL})$. The mixture was cooled with ice bath. To the mixture was added a portion of TMSI solution $(1 \mathrm{~mL})$, prepared from TMSI $(0.45 \mathrm{~mL}, 20.0 \mathrm{mmol})$ in $\mathrm{CHCl}_{3}(4.5 \mathrm{~mL})$. It turned pale white. TLC $\left(\mathrm{CH}_{3} \mathrm{OH} / \mathrm{CHCl}_{3}\right.$ 1:19) indicated a quick consumption of the starting material $\left(\mathrm{R}_{f}=0.93\right)$ and formation of a single Bocremained intermediate $\left(\mathrm{R}_{f}=0.52\right)$ and the product 5-BT $4\left(\mathrm{R}_{f}=0.06\right)$. The reaction should be terminated when the single Boc-remained intermediate was almost consumed, about 30 min post reaction. A solution of $\mathrm{NEt}_{3}(0.2 \mathrm{~mL})$ and $\mathrm{CH}_{3} \mathrm{OH}(0.9 \mathrm{~mL})$ was added for quenching. The mixture was partitioned between $\mathrm{CHCl}_{3}$ $(12 \mathrm{~mL})$ and satd. $\mathrm{NaHCO}_{3(\mathrm{aq})}(4 \mathrm{~mL})$. Organic layer was collected and the aqueous layer was extracted with $\mathrm{CHCl}_{3}$ (4 mL). The organic layers combined were dried over $\mathrm{MgSO}_{4}$ and filtered through gravitational filtration to give a residue in $130 \mathrm{mg}$ followed by purification through flash chromatography with eluents of $\mathrm{CH}_{3} \mathrm{OH} / \mathrm{CH}_{2} \mathrm{Cl}_{2}$ 1:29 to afford a plastic odor pale yellow gel 5-BT 4 in 75\% yield (40.3 mg, $0.12 \mathrm{mmol}$ ). ${ }^{1} \mathrm{H}-\mathrm{NMR}\left(500 \mathrm{MHz}, \mathrm{CDCl}_{3}\right) \delta 1.34(\mathrm{~s}, 12 \mathrm{H}, \mathrm{Bpin}), 3.10\left(\mathrm{dd}, J_{1^{\prime} \mathrm{a}, 1^{\prime} \mathrm{b}}=14.5 \mathrm{~Hz}, J_{1^{\prime} \mathrm{a}, 2^{\prime}}=7.5 \mathrm{~Hz}, 1 \mathrm{H}, \mathrm{H}-1^{\prime} \mathrm{a}\right)$, $3.27\left(\mathrm{dd}, J_{1 \mathrm{~b}^{\prime}, 1^{\prime} \mathrm{a}^{\prime}}=14.5 \mathrm{~Hz}, J_{1 \mathrm{~b}^{\prime}, 2^{\prime}}=5.0 \mathrm{~Hz}, 1 \mathrm{H}, \mathrm{H}-1 \mathrm{~b}^{\prime}\right), 3.71\left(\mathrm{~s}, 3 \mathrm{H}, \mathrm{COOCH}_{3}\right), 3.84\left(\mathrm{dd}, J_{2^{\prime}}, 1 \mathrm{a}^{\prime}=7.5 \mathrm{~Hz}, J_{2^{\prime}}\right.$, $\left.1 \mathrm{~b}^{\prime}=5.0 \mathrm{~Hz}, 1 \mathrm{H}, \mathrm{H}-2^{\prime}\right), 7.05(\mathrm{~s}, 1 \mathrm{H}$, arom-H), $7.33(\mathrm{~d}, J=8.0 \mathrm{~Hz}, 1 \mathrm{H}$, arom-H), $7.62(\mathrm{~d}, J=8.0 \mathrm{~Hz}, 1 \mathrm{H}$, 
arom-H), 8.09 (s, $1 \mathrm{H}$, arom-H), 8.15 (bs, $1 \mathrm{H}$, arom-NH); ${ }^{13} \mathrm{C}-\mathrm{NMR}\left(125 \mathrm{MHz}, \mathrm{CDCl}_{3}\right) \delta 24.86\left(\mathrm{CH}_{3}, \mathrm{Bpin}\right)$, $24.93\left(\mathrm{CH}_{3}, \mathrm{Bpin}\right), 30.36\left(\mathrm{CH}_{2}, \mathrm{C}-1^{\prime}\right), 52.02\left(\mathrm{CH}_{3}, \mathrm{CO}_{2} \underline{\mathrm{CH}} 3\right), 54.65\left(\mathrm{CH}, \mathrm{C}-2^{\prime}\right), 83.48(\mathrm{C}, \mathrm{Bpin}), 110.60$ $(\mathrm{CH}, \operatorname{arom}), 111.66(\mathrm{C}$, arom), $123.02(\mathrm{CH}$, arom $), 125.53(\mathrm{CH}$, arom $), 127.18(\mathrm{C}$, arom), $128.43(\mathrm{CH}$, arom), $138.35\left(\mathrm{C}\right.$, arom), $175.57\left(\mathrm{C}, \mathrm{CO}_{2} \mathrm{CH}_{3}\right)$; Analysis for $\mathrm{C}_{18} \mathrm{H}_{25} \mathrm{BN}_{2} \mathrm{O}_{4}$ : calculated for $[\mathrm{M}+\mathrm{H}]^{+}(\mathrm{m} / \mathrm{z})$ $=344.2022(24.8 \%), 345.1986(100.0 \%), 345.2055$ (4.8\%), $346.2019(19.4 \%), 347.2053(1.8 \%) ;[2 \mathrm{M}+\mathrm{H}]^{+}$ $(\mathrm{m} / \mathrm{z})=689.3893$; ESI-Q-TOF HR-ESI-MS found: $[\mathrm{M}+\mathrm{H}]^{+}=344.2021(24.0 \%), 345.1988(100.0 \%)$, $346.2020(19.5 \%), 347.2042(2.1 \%) ;[2 \mathrm{M}+\mathrm{H}]^{+}=689.3911, \delta=-0.58 \mathrm{ppm}$. 2-(acetylamino)acrylic acid $\mathbf{8}^{2,3}$

Anhydrous $\mathrm{CuSO}_{4}$ was dried in oven at $120{ }^{\circ} \mathrm{C}$ for one week. The three-bottomed flask of Soxhlet extraction assembly was charged with acetamide $(10.0 \mathrm{~g}, 0.17 \mathrm{~mol}, 1 \mathrm{eq})$, pyruvic acid $(30.0 \mathrm{~mL}, 0.4 \mathrm{~mol}$, $2.5 \mathrm{eq})$ and toluene $(300 \mathrm{~mL})$, sequentially. The mixture became clear upon reflux at $130{ }^{\circ} \mathrm{C}$. After $30 \mathrm{~min}$, white solid was precipitated. When the paper tube became deteriorated and colored (c.a. 40 min post reaction), it was replaced by a new paper tube along with fillings of $\mathrm{CuSO}_{4}$. After the second replacement and the reaction was allowed for additional $10 \mathrm{~min}$, the mixture was filtered through Buechner. The yellow solid residue was washed with acetone followed by concentration under reduced pressure to give a pale yellow solid 8 in a $55 \%$ yield $(12.0 \mathrm{~g}, 0.09 \mathrm{~mol})$ with a maltose-like sweet smell with $\mathrm{mp}$ of $188-190^{\circ} \mathrm{C}$ $\left(198-200^{\circ} \mathrm{C}\right)^{4}$.

${ }^{1} \mathrm{H}-\mathrm{NMR}\left(500 \mathrm{MHz}, \mathrm{DMSO}-\mathrm{d}_{6}^{2}\right) \delta 2.01\left(\mathrm{~s}, 3 \mathrm{H}, \mathrm{COC}_{3}\right), 5.65$ (s, 1H, vinyl), 6.22 (s, 1H, vinyl), 9.07 (s, $1 \mathrm{H}$,

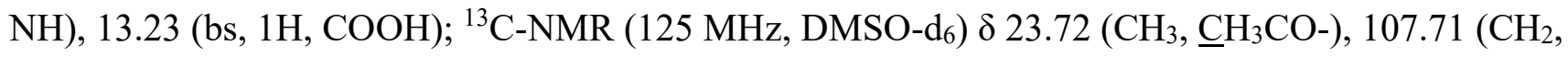
vinyl), 133.17 (C, vinyl), 164.97 (C, carbonyl), 169.24 (C, carbonyl); Analysis for $\mathrm{C}_{5} \mathrm{H}_{7} \mathrm{NO}_{3}$ : calculated for $[\mathrm{M}+\mathrm{Na}]^{+}(\mathrm{m} / \mathrm{z})=152.0324(100.0 \%), 153.0357(5.4 \%)$, ESI-Q-TOF HR-ESI-MS found: $[\mathrm{M}+\mathrm{Na}]^{+}=$ $152.0316(8.0 \%), 153.0354(0.5 \%), \delta=-5.3 \mathrm{ppm}$.

2-acetamidoacrylate methyl ester $\mathbf{9}^{5,6}$

A three-necked round-bottomed flask (1L) that was charged with $\mathrm{K}_{2} \mathrm{CO}_{3}(14.8 \mathrm{~g}, 0.1 \mathrm{~mol}$, $2.6 \mathrm{eq})$ was dried in oven at $120^{\circ} \mathrm{C}$ for $24 \mathrm{~h}$. To this mixture that has been cooled down to rt was added 8 ( $5.4 \mathrm{~g}, 41.9$ mmol, 1 eq) and acetone $(300 \mathrm{~mL})$, sequentially. A portion $(25 \mathrm{~mL})$ of a mixture of $\mathrm{CH}_{3} \mathrm{I}(7.5 \mathrm{~mL}, 0.1 \mathrm{~mol}, 3$ eq) in acetone $(40 \mathrm{~mL})$ was added to the above flask. The mixture was refluxed at $75-85{ }^{\circ} \mathrm{C}$. While the solution turned dark yellow, TLC $\left(\mathrm{CH}_{3} \mathrm{OH} / \mathrm{CH}_{2} \mathrm{Cl}_{2} 2: 8\right)$ indicated consumption of $\mathbf{8}\left(\mathrm{R}_{f}=0.16\right)$ and 
formation of product $9\left(\mathrm{R}_{f}=0.92\right)$. After $4 \mathrm{~h}$, a portion of additional acetone $(50 \mathrm{~mL})$ and the solution of $\mathrm{CH}_{3} \mathrm{I} /$ acetone $(15 \mathrm{~mL})$ was added. At $22 \mathrm{~h}$ post reaction, the mixture was filtered through suction with celite pad to afford a pale orange filtrate $(7.6 \mathrm{~g})$. Further purification using flash chromatography with eluents of EtOAc/ $n$-hexane 2:8 provided a colorless viscous foam 9 in a $93 \%$ yield (5.6 g, $39.2 \mathrm{mmol}$ ).

${ }^{1} \mathrm{H}-\mathrm{NMR}\left(500 \mathrm{MHz}, \mathrm{CDCl}_{3}{ }^{5}\right) \delta 2.10\left(\mathrm{~s}, 3 \mathrm{H}, \mathrm{CH}_{3} \mathrm{CO}-\right), 3.81\left(\mathrm{~s}, 3 \mathrm{H},-\mathrm{COOCH}_{3}\right), 5.85\left(\mathrm{~d}, J_{1 \mathrm{a}, 1 \mathrm{~b}}=1.0 \mathrm{~Hz}, 1 \mathrm{H}\right.$, vinyl), $6.56\left(\mathrm{~d}, J_{1 \mathrm{~b}, 1 \mathrm{a}}=1.0 \mathrm{~Hz}, 1 \mathrm{H}\right.$, vinyl), $7.71(\mathrm{bs}, 1 \mathrm{H}, \mathrm{NH}) ;{ }^{13} \mathrm{C}-\mathrm{NMR}\left(125 \mathrm{MHz}, \mathrm{CDCl}_{3}\right)^{5} \delta 24.56\left(\mathrm{CH}_{3}\right.$, $\left.\underline{\mathrm{CH}}_{3} \mathrm{CO}-\right), 52.89\left(\mathrm{CH}_{3},-\mathrm{COOCH}_{3}\right), 108.64\left(\mathrm{CH}_{2}\right.$, vinyl), 130.83 (C, vinyl), 164.55 (C, carbonyl), 168.77 (C, carbonyl); Analysis for $\mathrm{C}_{6} \mathrm{H}_{9} \mathrm{NO}_{3}$ : calculated for $[\mathrm{M}+\mathrm{H}]^{+}(\mathrm{m} / \mathrm{z})=144.0661$ (100.0\%), 145.0694 (6.5\%), ESIQ-TOF HR-ESI-MS found: $[\mathrm{M}+\mathrm{H}]^{+}=144.0655(100.0 \%), 145.0686(7.1 \%), \delta=-4.2 \mathrm{ppm}$.

5-bromo-3-(2R,2S)(2-N-acetyl)tryptophan methyl ester 10

Compound 9 (4.58 g, $32.2 \mathrm{mmol}, 1 \mathrm{eq})$ was evaporated with toluene (10 mL) twice. A two-necked round-bottomed flask was charged with 5-bromoindole (9.25 g, $47.4 \mathrm{mmol}, 1.5 \mathrm{eq})$ and $\mathrm{CH}_{2} \mathrm{Cl}_{2}(25 \mathrm{~mL})$, sequentially. Compound 9 in $\mathrm{CH}_{2} \mathrm{Cl}_{2}(10 \mathrm{~mL})$ along with its washings of $\mathrm{CH}_{2} \mathrm{Cl}_{2}(10 \mathrm{~mL})$ were transferred to the flask, sequentially. The clear pale yellow flask was cooled to $0{ }^{\circ} \mathrm{C}$ using ice bath. A mixture of EtAlCl 2 in heptane $(0.9 \mathrm{M}, 70 \mathrm{~mL}, 63.0 \mathrm{mmol}, 2 \mathrm{eq})$ was added over a 3-min time course, while the solution turned red. After $10 \mathrm{~min}$, the ice bath was removed and the mixture was stirred at $\mathrm{rt}$. TLC (acetone $/ n$-hexane $=3 / 7)$ indicated consumption of compound $9\left(\mathrm{R}_{f}=0.64\right)$ and formation of the product $\mathbf{1 0}\left(\mathrm{R}_{f}=0.22\right)$. After $1 \mathrm{~h}$, the mixture was transferred to extraction funnel through the transferring with EtOAc $(100 \mathrm{~mL})$. The mixture was washed with satd. $\mathrm{Na}_{2} \mathrm{CO}_{3}$ (aq., $50 \mathrm{~mL}$ ) while the bubbling evolved for 3 min. Additional satd. $\mathrm{Na}_{2} \mathrm{CO}_{3}$ (aq., $25 \mathrm{~mL}$ ) was added. The unseparated layers became separable upon adding satd. $\mathrm{NaCl}$ (aq., $20 \mathrm{~mL}$ ). The above pale yellow layer was collected. The aqueous milky precipitated layer was partitioned using additional EtOAc $(100 \mathrm{~mL})$ and satd. $\mathrm{NaCl}(20 \mathrm{~mL}$, aq.). The inseparable mixture became separable using additional EtOAc $(20 \mathrm{~mL})$. The organic layers combined were dried over $\mathrm{MgSO}_{4}$. After gravitational filtration, the filtrate was concentrated under reduced pressure to give a residue $(12.5 \mathrm{~g})$. Followed by flash chromatography with gradient mode of eluents of EtOAc/ $n$-hexane 5:5 $\rightarrow$ 6:4 $\rightarrow$ 7:3, a viscous yellow gum 10 was obtained in $85 \%$ yield (9.22 g, $27.2 \mathrm{mmol})$. HPLC analysis was carried out by injection of an aliquot from compound $\mathbf{1 0}$ in $\mathrm{EtOH}(0.7 \mathrm{mg} / 1.0 \mathrm{~mL})$. The HPLC conditions were performed using a RP- 
HPLC column of CHIRALCEL® OD-RH in a dimension of 4.6 x $150 \mathrm{~mm}$ filled with particles that have a diameter in $5 \mathrm{um}$. Elution was conditioned by using a gradient mode of $\mathrm{H}_{2} \mathrm{O} / \mathrm{EtOH}$ 80:20 $\rightarrow$ 70:30 from 0 to $10 \mathrm{~min}, \mathrm{H}_{2} \mathrm{O} / \mathrm{EtOH}$ 70:30 from 10 to $60 \mathrm{~min}, \mathrm{H}_{2} \mathrm{O} / \mathrm{EtOH} 70: 30 \rightarrow 60: 40$ from 60 to 70 min and 60:40 from 70 to $80 \mathrm{~min}$. The flow rate was set at $0.7 \mathrm{~mL} / \mathrm{min}$ from 0 to $60 \mathrm{~min}$ and $0.6 \mathrm{~mL} / \mathrm{min}$ from 60 to 80 min. UV detector was set at $\lambda_{\text {abs }}=254 \mathrm{~nm}$. The two peaks at $t_{R 1}=71.37 \mathrm{~min}$ and $t_{R 2}=75.43$ were analyzed using Origin software to provide individual integral for ratio calculation.

${ }^{1} \mathrm{H}-\mathrm{NMR}\left(500 \mathrm{MHz}, \mathrm{CDCl}_{3}\right) \delta 1.97\left(\mathrm{~s}, 3 \mathrm{H}, \mathrm{CH}_{3} \mathrm{CO}\right), 3.23\left(\mathrm{dd}, J_{1 \mathrm{a}^{\prime}, 1 \mathrm{~b}^{\prime}}=15.0, J_{1 \mathrm{a}^{\prime}, 2^{\prime}}=5.0 \mathrm{~Hz}, 1 \mathrm{H}, \mathrm{H}-1 \mathrm{a}^{\prime}\right), 3.29$ $\left(\mathrm{dd}, J_{1 \mathrm{~b}^{\prime}, 1 \mathrm{a}^{\prime}}=15.0, J_{1 \mathrm{~b}^{\prime} \mathrm{2}^{\prime}}=5.5 \mathrm{~Hz}, 1 \mathrm{H}, \mathrm{H}-1 \mathrm{~b}^{\prime}\right), 3.70\left(\mathrm{~s}, 3 \mathrm{H}, \mathrm{COOCH}_{3}\right), 4.91\left(\mathrm{ddd}, J_{2^{\prime}, \mathrm{NH}}=7.5, J_{2^{\prime}, 1 \mathrm{~b}^{\prime}}=5.5\right.$, $\left.J_{2^{\prime}, 1 \mathrm{a}^{\prime}}=5.0 \mathrm{~Hz}, 1 \mathrm{H}, \mathrm{H}-2^{\prime}\right), 6.01\left(\mathrm{~d}, J_{\mathrm{NH}, 2^{\prime}}=7.5 \mathrm{~Hz}, 1 \mathrm{H}, \mathrm{C}-2^{\prime}-\mathrm{NHCO}\right), 6.95(\mathrm{~d}, J=2.5 \mathrm{~Hz}, 1 \mathrm{H}$, arom $), 7.20(\mathrm{~d}$,

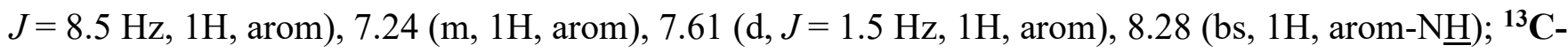

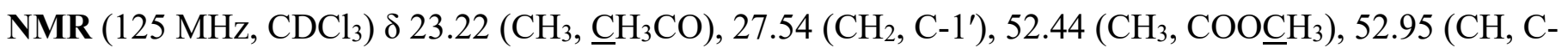
2'), $109.92(\mathrm{C}$, arom $), 112.74(\mathrm{CH}$, arom $), 112.99(\mathrm{C}$, arom $), 121.35(\mathrm{CH}$, arom $), 123.96(\mathrm{CH}$, arom $), 125.09$ (CH, arom), 129.49 (C, arom), 134.70 (C, arom), 169.73 (C, carbonyl), 172.20 (C, carbonyl); Analysis for $\mathrm{C}_{14} \mathrm{H}_{15} \mathrm{BrN}_{2} \mathrm{O}_{3}$ : calculated for $[\mathrm{M}+\mathrm{H}]^{+}(\mathrm{m} / \mathrm{z})=339.0344$ (100.0\%), 340.0378 (15.1\%), 341.0324 (97.3\%), 341.0411 (1.1\%), 342.0357 (14.7\%), 343.0391 (1.0\%), ESI-Q-TOF HR-ESI-MS found: $[\mathrm{M}+\mathrm{H}]^{+}=339.0340$ (100.0\%), $340.0372(16.2 \%), 341.0320(95.9 \%), 342.0354(12.5 \%), \delta=-1.2 \mathrm{ppm}$.

3-(2R,2S)-(2-N-acetyl)-1,N-diBoc-5-bromotryptophan methyl ester 11

A round-bottomed flask $(250 \mathrm{~mL})$ was charged with DMAP $(1.50 \mathrm{~g}, 12.3 \mathrm{mmol}, 1.5 \mathrm{eq})$, a solution of $\mathrm{Boc}_{2} \mathrm{O}(6.1 \mathrm{~mL}, 26.5 \mathrm{mmol}, 3 \mathrm{eq})$ in $\mathrm{CH}_{3} \mathrm{CN}(20 \mathrm{~mL})$ and a mixture of compound $\mathbf{1 0}$ (2.80 g, $8.3 \mathrm{mmol}, 1$ eq) in $\mathrm{CH}_{3} \mathrm{CN}(30 \mathrm{~mL})$, sequentially. While the mixture turned pale yellow and evolved gas, TLC (acetone/n-hexane 3:7) indicated consumption of starting material $10\left(\mathrm{R}_{f}=0.24\right)$ and formation of the product $11\left(\mathrm{R}_{f}=0.76\right)$. After $30 \mathrm{~min}$, a second portions of DMAP (500 mg, $\left.4.1 \mathrm{mmol}, 0.5 \mathrm{eq}\right)$ and a solution of $\mathrm{Boc}_{2} \mathrm{O}(1.7 \mathrm{~mL}, 7.4 \mathrm{mmol}, 0.8 \mathrm{eq})$ in $\mathrm{CH}_{3} \mathrm{CN}(10 \mathrm{~mL})$ were added, sequentially. At 2.5 h post reaction, the dark orange mixture were concentrated under reduced pressure. The residue was dissolved in EtOAc (30 $\mathrm{mL})$ and transferred to extraction funnel. After washing with $\mathrm{HCl}_{(\mathrm{aq})}(1 \mathrm{~N}, 10 \mathrm{~mL})$ twice and $\mathrm{Na}_{2} \mathrm{CO}_{3(\mathrm{aq})}(10$ $\mathrm{mL}$ ), the organic layer was dried with $\mathrm{MgSO}_{4}$ followed by gravitational filtration. The filtrate was 
concentrated under reduced pressure to give a dark orange residue $(5.1 \mathrm{~g})$. After purification with flash chromatography with gradient mode of eluents of EtOAc/ $n$-hexane $1: 19 \rightarrow 1: 9 \rightarrow 1: 7$, a pleasant odor of pale yellow viscous liquid 11 was obtained in $89 \%$ yield (3.97 g, $7.37 \mathrm{mmol})$.

${ }^{1} \mathrm{H}-\mathrm{NMR}\left(500 \mathrm{MHz}, \mathrm{CDCl}_{3}\right) \delta 1.33$ (s, 9H, $N$ - $\underline{\mathrm{Boc}}$ ), 1.62 (s, 9H, $N$-Boc), 2.35 (s, 3H, $\left.\mathrm{CH}_{3} \mathrm{CO}\right), 3.24$ (dd, $\left.J_{1 \mathrm{a}^{\prime}, 1 \mathrm{~b}^{\prime}}=15.0, J_{1 \mathrm{a}^{\prime}, 2^{\prime}}=9.5 \mathrm{~Hz}, 1 \mathrm{H}, \mathrm{H}-1 \mathrm{a}^{\prime}\right), 3.47\left(\mathrm{dd}, J_{1 \mathrm{~b}^{\prime}, 1 \mathrm{a}^{\prime}}=15.0, J_{1 \mathrm{~b}^{\prime}, 2^{\prime}}=5.0 \mathrm{~Hz}, 1 \mathrm{H}, \mathrm{H}-1 \mathrm{~b}^{\prime}\right), 3.73(\mathrm{~s}, 3 \mathrm{H}$, $\left.\mathrm{COOCH}_{3}\right), 5.38\left(\mathrm{dd}, J_{2^{\prime}, 1 \mathrm{a}^{\prime}}=9.5, J_{2^{\prime}}, 1 \mathrm{~b}^{\prime}=5.0 \mathrm{~Hz}, 1 \mathrm{H}, \mathrm{H}-2^{\prime}\right), 7.34(\mathrm{~s}, 1 \mathrm{H}, \mathrm{H}-2), 7.36(\mathrm{dd}, J=8.5, J=1.5 \mathrm{~Hz}$, 1H, H-6), 7.60 (d, $J=1.5 \mathrm{~Hz}, 1 \mathrm{H}, \mathrm{H}-4), 7.96$ (d, $J=8.5 \mathrm{~Hz}, 1 \mathrm{H}, \mathrm{H}-7) ;{ }^{13} \mathrm{C}-\mathrm{NMR}\left(125 \mathrm{MHz}, \mathrm{CDCl}_{3}\right) \delta 24.88$ $\left(\mathrm{CH}_{2}, \mathrm{C}-1^{\prime}\right), 26.55\left(\mathrm{CH}_{3}, \underline{\mathrm{C}} \mathrm{H}_{3} \mathrm{CO}\right), 27.66\left(\mathrm{CH}_{3}, \mathrm{CO}_{2} \mathrm{C}\left(\underline{\mathrm{C}} \mathrm{H}_{3}\right)_{3}\right), 28.14\left(\mathrm{CH}_{3}, \mathrm{CO}_{2} \mathrm{C}\left(\underline{\mathrm{CH}}_{3}\right)_{3}\right), 52.37\left(\mathrm{CH}_{3}\right.$, $\left.\mathrm{COO}^{-} H_{3}\right), 56.45\left(\mathrm{CH}, \mathrm{C}-2^{\prime}\right), 84.03\left(\mathrm{C}, \mathrm{CO}_{2} \underline{\mathrm{C}}\left(\mathrm{CH}_{3}\right)_{3}\right), 84.24\left(\mathrm{C}, \mathrm{CO}_{2} \underline{\mathrm{C}}\left(\mathrm{CH}_{3}\right)_{3}\right), 115.97(\mathrm{C}$, arom $), 116.74$ $(\mathrm{CH}, \operatorname{arom}), 121.55(\mathrm{CH}$, arom $), 125.28(\mathrm{CH}, \operatorname{arom}), 127.21(\mathrm{CH}$, arom $), 132.23(\mathrm{C}$, arom), $134.12(\mathrm{C}$, arom), $149.19\left(\mathrm{C}, \underline{\mathrm{CO}}_{2} \mathrm{C}\left(\mathrm{CH}_{3}\right)_{3}\right), 151.99\left(\mathrm{C}, \underline{\mathrm{CO}}_{2} \mathrm{C}\left(\mathrm{CH}_{3}\right)_{3}\right), 170.39$ (C, carbonyl), 172.73 (C, carbonyl); Analysis for $\mathrm{C}_{24} \mathrm{H}_{31} \mathrm{BrN}_{2} \mathrm{O}_{7}$ : calculated for $[\mathrm{M}+\mathrm{Na}]^{+}(\mathrm{m} / \mathrm{z})=561.1212(100.0 \%), 562.1246(26.0 \%)$, 563.1192 (97.3\%), 564.1225 (25.3\%), ESI-Q-TOF HR-ESI-MS found: $[\mathrm{M}+\mathrm{Na}]^{+}=561.1241(100.0 \%)$, $562.1273(22.4 \%), 563.1223(98.2 \%), 564.1256(21.2 \%), \delta=5.1 \mathrm{ppm}$.

3-(2R,2S)(2-N-acetyl)-1,N-diBoc-5-boronopinacoltryptophan methyl ester (DBA-5-BT) 2

The borylation is the same as that described for TB-6-BT 5. Reagents such as $\mathrm{B}_{2} \mathrm{Pin}_{2}(2.16 \mathrm{~g}, 8.2 \mathrm{mmol}$, 2 eq), 11 (2.20 g, $4.1 \mathrm{mmol}, 1$ eq), Pd(dppf)Cl 2 (310 mg, 0.4 mmol, 0.1 eq), DMSO (45 mL) and KOAc $(1.60 \mathrm{~g}, 16.3 \mathrm{mmol}, 4 \mathrm{eq})$ were used. TLC (EtOAc/n-hexane 2:8) indicated consumption of starting material $\left(\mathrm{R}_{f}=0.66\right)$ and formation of the product DBA-5-BT $2\left(\mathrm{R}_{f}=0.60\right)$. Temperature were set as following: $0 \sim 19$ $\min$ at $120^{\circ} \mathrm{C}, 19 \sim 30 \mathrm{~min}$ at $104^{\circ} \mathrm{C}, 30 \sim 50 \mathrm{~min}$ at $90^{\circ} \mathrm{C}$ and $50 \sim 60 \mathrm{~min}$ at $100^{\circ} \mathrm{C}$. After partition between $\mathrm{CH}_{2} \mathrm{Cl}_{2}(50 \mathrm{~mL})$ and $0.2 \mathrm{~N} \mathrm{HCl}_{(\mathrm{aq})}(20 \mathrm{~mL})$, the organic layer still contained DMSO. The mixture was concentrated under reduced pressure to give the residue following by dissolving in EtOAc $(50 \mathrm{~mL})$ for washing with satd. $\mathrm{NaCl}$ (aq., $15 \mathrm{~mL}$ ) twice. After gravitational filtration of the organic layer and dryness, the filtrate was concentrated under reduced pressure to give a residue. It was purified through flash chromatography with gradient a mode of eluents of EtOAc/n-hexane $1: 19 \rightarrow 1: 9 \rightarrow 1: 7$ to provide a pleasant odor, pale yellow and viscous liquid DBA-5-BT 2 in $64 \%$ yield (1.52 g, $2.6 \mathrm{mmol})$. ${ }^{1} \mathrm{H}-\mathrm{NMR}\left(500 \mathrm{MHz}, \mathrm{CDCl}_{3}\right) \delta 1.25$ (s, 9H, N-Boc), 1.34 (s, 12H, Bpin), 1.62 ((s, 9H, N-Boc), 2.34 (s, 3H, 
$\left.\mathrm{CH}_{3} \mathrm{CO}\right), 3.34\left(\mathrm{dd}, J_{1 \mathrm{a}^{\prime}, 1 \mathrm{~b}^{\prime}}=15.0, J_{1 \mathrm{a}^{\prime} 2^{\prime}}=10.5 \mathrm{~Hz}, 1 \mathrm{H}, \mathrm{H}-1 \mathrm{a}^{\prime}\right), 3.52\left(\mathrm{dd}, J_{1 \mathrm{~b}^{\prime}, 1 \mathrm{a}^{\prime}}=15.0, J_{1 \mathrm{~b}^{\prime}, 2^{\prime}}=5.0 \mathrm{~Hz}, 1 \mathrm{H}, \mathrm{H}-\right.$ $\left.1 \mathrm{~b}^{\prime}\right), 3.75\left(\mathrm{~s}, 3 \mathrm{H}, \mathrm{COOCH}_{3}\right), 5.38\left(\mathrm{dd}, J_{2^{\prime}, 1 \mathrm{a}^{\prime}}=10.5, J_{2^{\prime}, 1 \mathrm{~b}^{\prime}}=5.0 \mathrm{~Hz}, 1 \mathrm{H}, \mathrm{H}-2^{\prime}\right), 7.30(\mathrm{~s}, 1 \mathrm{H}$, arom-H), $7.72(\mathrm{~d}$, $J=8.0 \mathrm{~Hz}, 1 \mathrm{H}$, arom-H), $7.92\left(\mathrm{~s}, 1 \mathrm{H}\right.$, arom-H), $8.08\left(\mathrm{~d}, J=8.0 \mathrm{~Hz}, 1 \mathrm{H}\right.$, arom-H); ${ }^{13} \mathrm{C}-\mathrm{NMR}(125 \mathrm{MHz}$, $\left.\mathrm{CDCl}_{3}\right) \delta 24.64\left(\mathrm{CH}_{2}, \mathrm{C}-1^{\prime}\right), 24.85\left(\mathrm{CH}_{3}, \mathrm{Bpin}\right), 24.91\left(\mathrm{CH}_{3}, \mathrm{Bpin}\right), 26.58\left(\mathrm{CH}_{3}, \mathrm{CH}_{3} \mathrm{CO}\right), 27.56\left(\mathrm{CH}_{3}, N-\right.$ Boc), $28.17\left(\mathrm{CH}_{3}, N\right.$-Boc), $52.32\left(\mathrm{CH}_{3}, \mathrm{COO}^{-} H_{3}\right), 56.84\left(\mathrm{CH}, \mathrm{C}-2^{\prime}\right), 83.61$ (C, $N$-Boc), 83.67 (C, $N$-Boc), 83.91 (C, Bpin), 114.57 (CH, arom), 116.99 (C, arom), 124.07 (CH, arom), 125.91 (CH, arom), 130.05 (C, arom), 130.87 (CH, arom), 137.53 (C, arom), 149.46 (C, $N$-Boc), 152.04 (C, $N$-Boc), 170.59 (C, carbonyl), 172.85 (C, carbonyl); Analysis for $\mathrm{C}_{30} \mathrm{H}_{43} \mathrm{BN}_{2} \mathrm{O}_{9}$ : calculated for $[\mathrm{M}+\mathrm{Na}]^{+}(\mathrm{m} / \mathrm{z})=608.2996(24.8 \%)$, 609.2959 (100.0\%), 610.2993 (32.4\%), ESI-Q-TOF HR-ESI-MS found: $[\mathrm{M}+\mathrm{Na}]^{+}=608.2998(21.4 \%)$, $609.2964(100 \%), 610.2995(33.3 \%), \delta=0.82 \mathrm{ppm}$.

3-(2R,2S)(2-N-acetyl)-5-boronopinacoltryptophan methyl ester NA-5-BT 3

The similar procedure as that described for preparation of TB-6-BT 5 was used, including the reagents such as 5-bromotryptophan derivative $\mathbf{1 0}$ (2.01 g, $5.9 \mathrm{mmol}, 1 \mathrm{eq}), \mathrm{B}_{2} \mathrm{Pin}_{2}(3.28 \mathrm{~g}, 12.9 \mathrm{mmol}, 2 \mathrm{eq})$, $\mathrm{Pd}(\mathrm{dppf}) \mathrm{Cl}_{2}(230 \mathrm{mg}, 0.3 \mathrm{mmol}, 10 \% \mathrm{wt}$ eq. $)$, DMSO (45 mL) and KOAc (2.39 g, $\left.24.3 \mathrm{mmol}, 4 \mathrm{eq}\right)$. TLC $\left(\mathrm{CH}_{3} \mathrm{OH} / \mathrm{CH}_{2} \mathrm{Cl}_{2}\right.$ 1:19) indicated consumption of $\mathbf{1 0}\left(\mathrm{R}_{f}=0.56\right)$ in half and formation of product NA-5-BT 3 $\left(\mathrm{R}_{f}=0.62\right)$. After $1 \mathrm{~h}$, a ratio of $1: 1$ for the starting material 10 to the product NA-5-BT 3 was observed. The mixture was partitioned between $\mathrm{CH}_{2} \mathrm{Cl}_{2}(20 \mathrm{~mL})$ and $0.1 \mathrm{~N} \mathrm{HCl}_{(\mathrm{aq})}(15 \mathrm{~mL})$. The aqueous layer was washed with $\mathrm{CH}_{2} \mathrm{Cl}_{2}(20 \mathrm{~mL})$ twice followed by collection of the organic layers, dryness, filtration and concentration under reduced pressure to give the residue. After flash chromatography using eluents of acetone $/ n$-hexane 3.5:6.5, a plastic-like odor pale yellow foam mixture was obtained in an amount of $1.95 \mathrm{~g}$ (5.05 mmol). Repeating the same borylation procedure but reducing the reagent amounts to half an equivalent, a plasticlike odor pale yellow foam-like solid NA-5-BT 3 was obtained in total 70\% yield (1.60 g, 4.14 mmol). Analytical data matched the literature values. ${ }^{7}$

${ }^{1} \mathrm{H}-\mathrm{NMR}\left(500 \mathrm{MHz}, \mathrm{CDCl}_{3}\right) \delta 1.33$ (s, 12H, Bpin), 1.97 (s, 3H, $\left.\mathrm{CH}_{3} \mathrm{CO}\right), 3.30\left(\mathrm{dd}, J_{1 \mathrm{a}^{\prime}, 1 \mathrm{~b}^{\prime}}=15.0, J_{1 \mathrm{a}^{\prime}, 2^{\prime}}=5.0\right.$ $\left.\mathrm{Hz}, 1 \mathrm{H}, \mathrm{H}-1 \mathrm{a}^{\prime}\right), 3.37\left(\mathrm{dd}, J_{1 \mathrm{~b}^{\prime}, \mathrm{la}^{\prime}}=15.0, J_{1 \mathrm{~b}^{\prime}, 2^{\prime}}=5.0 \mathrm{~Hz}, 1 \mathrm{H}, \mathrm{H}-1 \mathrm{~b}^{\prime}\right), 3.71\left(\mathrm{~s}, 3 \mathrm{H}, \mathrm{COOCH}_{3}\right), 4.96\left(\mathrm{ddd}, J_{2^{\prime}, \mathrm{NH}}=\right.$ $\left.8.0 \mathrm{~Hz}, J_{2^{\prime}, 1 \mathrm{a}^{\prime}}=5.0, J_{2^{\prime}, 1 \mathrm{~b}^{\prime}}=5.0 \mathrm{~Hz}, 1 \mathrm{H}, \mathrm{H}-2^{\prime}\right), 6.01\left(\mathrm{~d}, J_{\mathrm{NH}, 2^{\prime}}=8.0 \mathrm{~Hz}, 1 \mathrm{H}, \mathrm{CH}_{3} \mathrm{CONH}\right), 6.94(\mathrm{~s}, 1 \mathrm{H}$, aromH), $7.32(\mathrm{~d}, J=8.0 \mathrm{~Hz}, 1 \mathrm{H}$, arom-H), $7.61(\mathrm{~d}, J=8.0 \mathrm{~Hz}, 1 \mathrm{H}, \operatorname{arom}-\mathrm{H}), 8.02(\mathrm{~s}, 1 \mathrm{H}$, arom-H), $8.22(\mathrm{~s}, 1 \mathrm{H}$, 


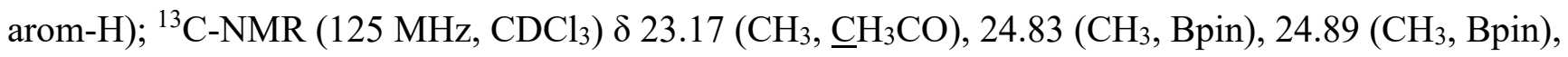
$27.61\left(\mathrm{CH}_{2}, \mathrm{C}-1^{\prime}\right), 52.31\left(\mathrm{CH}_{3}, \mathrm{COO}^{\prime} H_{3}\right), 53.01\left(\mathrm{CH}, \mathrm{C}-2^{\prime}\right), 83.48(\mathrm{C}, \mathrm{Bpin}), 110.68(\mathrm{CH}$, arom $), 110.71$ $(\mathrm{C}, \operatorname{arom}), 122.83(\mathrm{CH}$, arom $), 126.64(\mathrm{CH}, \operatorname{arom}), 127.43(\mathrm{C}, \operatorname{arom}), 128.31(\mathrm{CH}$, arom $), 138.22(\mathrm{C}$, arom $)$, $169.78\left(\mathrm{C}\right.$, carbonyl), $172.23\left(\mathrm{C}\right.$, carbonyl); Analysis for $\mathrm{C}_{20} \mathrm{H}_{27} \mathrm{BN}_{2} \mathrm{O}_{5}$ : calculated for $[\mathrm{M}+\mathrm{Na}]^{+}(\mathrm{m} / \mathrm{z})=$ 408.1947 (24.8\%), 409.1911 (100.0\%), 410.1944 (21.6\%), ESI-Q-TOF HR-ESI-MS found: $[\mathrm{M}+\mathrm{Na}]^{+}=$ 408.1947 (21.6\%), $409.1916(100.0 \%), 410.1944(19.4 \%), \delta=1.2 \mathrm{ppm}$

5-boronopinacol indole

Reagents, e.g. $\mathrm{B}_{2} \mathrm{Pin}_{2}$ (540 mg, $2.12 \mathrm{mmol}, 2$ eq), 5-bromoindole (230 mg, $\left.1.17 \mathrm{mmol}, 1 \mathrm{eq}\right)$, $\mathrm{Pd}(\mathrm{dppf}) \mathrm{Cl}_{2}$ (40 mg, $0.05 \mathrm{mmol}, 15 \% \mathrm{wt}$ eq.), DMSO (45 mL) and KOAc (420 mg, $\left.4.27 \mathrm{mmol}, 4 \mathrm{eq}\right)$ were used. TLC (EtOAc/n-hexane 3:7) indicated consumption of 5-bromoindole $\left(\mathrm{R}_{f}=0.66\right)$ and formation of product $\left(\mathrm{R}_{f}=0.68\right)$. Because of the formation of an unknown byproduct after $2 \mathrm{~h}\left(\mathrm{R}_{f}=0.34\right)$, the mixture was transferred to partitioned between EtOAc $(25 \mathrm{~mL})$ and satd. $\mathrm{NaCl}$ (aq., $8 \mathrm{~mL}) \times 3$. The organic layer was collected and dried with $\mathrm{MgSO}_{4}$. After gravitational filtration, the filtrate was concentrated under reduced pressure to give a residue $(1.3 \mathrm{~g})$. After flash chromatography (EtOAc/n-hexane 1:19 $\rightarrow 1: 9)$, a pale green solid with pleasant odor was obtained (190 mg). The sample was further recrystallized from $n$-hexane to give the product in $42 \%$ yield $(120 \mathrm{mg}, 0.49 \mathrm{mmol}) . \mathrm{mp}: 103-108{ }^{\circ} \mathrm{C}\left(91-92^{\circ} \mathrm{C}^{8}\right)$

${ }^{1} \mathrm{H}-\mathrm{NMR}\left(500 \mathrm{MHz}, \mathrm{CDCl}_{3}\right) \delta 1.36(\mathrm{~s}, 12 \mathrm{H}$, pinacol), $6.55(\mathrm{t}, J=2.0 \mathrm{~Hz}, 1 \mathrm{H}$, arom), $7.16(\mathrm{t}, J=2.0 \mathrm{~Hz}, 1 \mathrm{H}$, arom), 7.36 (d, $J=8.5 \mathrm{~Hz}, 1 \mathrm{H}$, arom), 7.63 (d, $J=8.5 \mathrm{~Hz}, 1 \mathrm{H}$,arom), 8.18 (s, 1H, arom), 8.23 (bs, 1H, arom$\mathrm{NH}) ;{ }^{13} \mathrm{C}-\mathrm{NMR}\left(125 \mathrm{MHz}, \mathrm{CDCl}_{3}\right) \delta 24.87\left(\mathrm{CH}_{3}, \mathrm{Bpin}\right), 83.43$ (C, Bpin), $103.13(\mathrm{CH}$, arom), $110.43(\mathrm{CH}$, arom), $124.13(\mathrm{CH}$, arom), $127.59(\mathrm{C}$, arom), $128.06(\mathrm{CH}, \operatorname{arom}), 128.63(\mathrm{CH}$, arom $), 137.81(\mathrm{C}$, arom); Analysis for $\mathrm{C}_{14} \mathrm{H}_{18} \mathrm{BNO}_{2}$ : calcd. $[\mathrm{M}+\mathrm{H}]^{+}(\mathrm{m} / \mathrm{z})=243.1545$ (24.8\%), 244.1509 (100.0\%), 245.1542 (15.1\%), ESI-Q-TOF HR-ESI-MS found: $[\mathrm{M}+\mathrm{H}]^{+}=243.1541$ (25.1\%), 244.1508 (100\%), 245.1540 $(14.3 \%), \delta=-0.4 \mathrm{ppm}$

6-nitro tryptophan $p$-12 and 4-nitro tryptophan $o-\mathbf{1 2}^{\mathbf{9}}$

A three-necked round-bottomed flask was charged with tryptophan $(31.1 \mathrm{~g}, 152 \mathrm{mmol})$, urea (420 mg, $6.84 \mathrm{mmol}, 0.045 \mathrm{eq})$ and glacial $\mathrm{AcOH}(380 \mathrm{~mL})$, sequentially. The mixture turned pasty and cold 
rendering the stirring difficult. The stirring was enabled by additional glacial $\mathrm{AcOH}(60 \mathrm{~mL})$ under warming the flask with heating gun. A solution containing $\mathrm{HNO}_{3}(7 \mathrm{~mL})$ and glacial $\mathrm{AcOH}(23 \mathrm{~mL})$ in a feeding funnel $(60 \mathrm{~mL})$ was added within $20 \mathrm{~min}$. The white suspension turned brown then yellow cloudy very shortly. Addition of another portion of $\mathrm{HNO}_{3}(3 \mathrm{~mL})$ and glacial $\mathrm{AcOH}(23 \mathrm{~mL})$ did not improve the dissolution. The mixture was then kept at $10{ }^{\circ} \mathrm{C}$ using an ice-cold wet towel. A further portion of $\mathrm{HNO}_{3}(13$ $\mathrm{mL})$ and glacial AcOH $(55 \mathrm{~mL})$ in a feeding funnel $(250 \mathrm{~mL})$ was added within $40 \mathrm{~min}$. After removing the clod wet towel, the solution turned into a smoothie. After $30 \mathrm{~min}$, the smoothie melted to form a brown solution; it became a pale orange precipitate in $6 \mathrm{~h}$. After a stirring for further $6 \mathrm{~h}$, the mixture was collected through gravitational filtration with a Buechner. The brown solid was further washed with cold $\mathrm{CHCl}_{3}(50$ $\mathrm{mL})$. The solid was concentrated under reduced pressure. The crude mixture was dissolved in hot water ( 80 $\mathrm{mL})$ at $80{ }^{\circ} \mathrm{C}$. A solution containing $\mathrm{Na}_{2} \mathrm{CO}_{3}(2.3 \mathrm{~g}, 22 \mathrm{mmol}, 0.5 \mathrm{eq})$ in $\mathrm{H}_{2} \mathrm{O}(20 \mathrm{~mL})$ was slowly added whereas a gas evolved violently. The mixture was concentrated under reduced pressure followed by distillation with $\mathrm{CH}_{3} \mathrm{OH}(20 \mathrm{~mL})$ three times to give a dark yellow solid in $41 \%$ yield $(15.4 \mathrm{~g})$.

${ }^{1} \mathrm{H}-\mathrm{NMR}\left(500 \mathrm{MHz}, \mathrm{CD}_{3} \mathrm{OD}\right) \delta 1.17\left(\mathrm{t}, J=7.0 \mathrm{~Hz}, 2 \mathrm{H}, \mathrm{NH}_{2}, \boldsymbol{p}-\mathbf{- 1 2}+\boldsymbol{o}-\mathbf{1 2}\right), 3.42\left(\mathrm{dd}, J_{1 a^{\prime}, 1 b^{\prime}}=15.5, J_{1 a^{\prime}, 2^{\prime}}=\right.$ $\left.7.0 \mathrm{~Hz}, 1 \mathrm{H}, \mathrm{H}-1 \mathrm{a}^{\prime}, \boldsymbol{p}-12\right), 3.50\left(\mathrm{dd}, J_{1 b^{\prime}, 1 a^{\prime}}=15.5, J_{1 b^{\prime} 2^{\prime}}=5.0 \mathrm{~Hz}, 1 \mathrm{H}, \mathrm{H}-1 \mathrm{~b}^{\prime}, \boldsymbol{p}-12\right), 3.60\left(\mathrm{dd}, J_{1 a^{\prime} l b^{\prime}}=15.5\right.$, $\left.J_{l a^{\prime}, 2^{\prime}}=7.0 \mathrm{~Hz}, 1 \mathrm{H}, \mathrm{H}-1 \mathrm{a}^{\prime}, \boldsymbol{o}-12\right), 3.77\left(\mathrm{dd}, J_{1 b^{\prime}, 1 a^{\prime}}=14.5, J_{l b^{\prime}, 2^{\prime}}=5.5 \mathrm{~Hz}, 1 \mathrm{H}, \mathrm{H}-1 \mathrm{~b}^{\prime}, \boldsymbol{o}-12\right), 4.12\left(\mathrm{ddd}, J_{2^{\prime}, N H}=\right.$ $\left.7.0, J_{2^{\prime}, 1 a^{\prime}}=7.0, J_{2^{\prime}, 1 b^{\prime}}=5.5 \mathrm{~Hz}, 1 \mathrm{H}, \mathrm{H}-2^{\prime}, \boldsymbol{o}-12\right), 4.29\left(\mathrm{ddd}, J_{2^{\prime}, N H}=7.0, J_{2^{\prime}, 1 a^{\prime}}=7.0, J_{2^{\prime}, 1 b^{\prime}}=5.0 \mathrm{~Hz}, 1 \mathrm{H}, \mathrm{H}-2^{\prime}\right.$, p-12), $7.27(\mathrm{t}, J=8.5 \mathrm{~Hz}, 1 \mathrm{H}$, arom, $\boldsymbol{o - 1 2}), 7.50$ (s, 1H, arom, o-12), 7.57 (s, 1H, arom, p-12), $7.73(\mathrm{~d}, J=$ $9.0 \mathrm{~Hz}, 1 \mathrm{H}$, arom, p-12), 7.79 (d, $J=8.0 \mathrm{~Hz}, 1 \mathrm{H}$, arom, $\boldsymbol{o - 1 2}), 7.95$ (d, 1H, arom, p-12), 7.97 (d, $J=7.5 \mathrm{~Hz}$, 1H, arom, $\boldsymbol{o - 1 2}$ ), 8.3 (s, 1H, arom, $\boldsymbol{p}$-12), 11.45 (bs, 1H, NH, $\boldsymbol{p}-12), 11.56$ (bs, 1H, NH, $\boldsymbol{o}$-12). Peak integral 
ratio of $\boldsymbol{p}$-12 vs. $\boldsymbol{o}-12$ was 3:1. ${ }^{13} \mathrm{C}-\mathrm{NMR}\left(125 \mathrm{MHz}, \mathrm{CD}_{3} \mathrm{OD}\right) \delta 27.12\left(\mathrm{CH}_{2}, \mathrm{C}-1^{\prime}\right), 54.48\left(\mathrm{CH}, \mathrm{C}-2^{\prime}\right), 109.40$

$(\mathrm{CH}$, arom $), 109.48(\mathrm{CH}, \operatorname{arom}), 115.45(\mathrm{CH}$, arom $), 119.31(\mathrm{CH}$, arom $), 132.05(\mathrm{CH}$, arom $), 133.10(\mathrm{C}$,

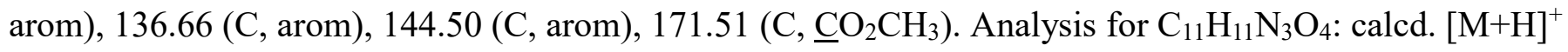
$(\mathrm{m} / \mathrm{z})=250.0828(100.0 \%), 251.0861(11.9 \%), 251.0798$ (1.1\%); ESI-Q-TOF HR-ESI-MS: found [M+H] $=250.0824(41.5 \%), 251.0847(5.2 \%), 252.0877(0.6 \%) . \delta=-1.6 \mathrm{ppm}$.

1,3-(2-N)diBoc-6-nitro tryptophan methyl ester $\boldsymbol{p}-13$ and 1,3-(2-N)diBoc-4-nitro tryptophan methyl ester $\boldsymbol{o - 1 3}$

A two-necked round-bottomed flask $(25 \mathrm{~mL})$ containing the methyl nitro tryptophan analog $12(20 \mathrm{mg}$, $0.08 \mathrm{mmol})$ in $\mathrm{CH}_{3} \mathrm{OH}(3 \mathrm{~mL})$ was stirred with ice bath under $\mathrm{N}_{2} . \mathrm{SOCl}_{2}(0.1 \mathrm{~mL}, 0.48 \mathrm{mmol}, 1.2 \mathrm{eq})$ was added. The ice bath was removed and the mixture was refluxed for $1 \mathrm{~h}$. TLC $\left(\mathrm{CH}_{3} \mathrm{OH} / \mathrm{CH}_{2} \mathrm{Cl}_{2}\right.$ 6:4) indicated consumption of the starting material $\left(\mathrm{R}_{f}=0.10\right)$ and formation of the ester intermediate $\left(\mathrm{R}_{f}=0.66\right)$. After concentration at $40{ }^{\circ} \mathrm{C}$ under reduced pressure, $\mathrm{CH}_{3} \mathrm{OH}(3 \mathrm{~mL})$ was added. Followed by addition of $\mathrm{Et}_{3} \mathrm{~N}$ $(0.08 \mathrm{~mL}, 1.1 \mathrm{mmol}, 14 \mathrm{eq})$ and $(\mathrm{Boc})_{2} \mathrm{O}(0.03 \mathrm{~mL}, 0.12 \mathrm{mmol}, 1.5 \mathrm{eq})$, sequentially, the mixture was stirred for $30 \mathrm{~min}$. The mixture was concentrated under reduced pressure. $\mathrm{CH}_{3} \mathrm{CN}(2 \mathrm{~mL}), \mathrm{DMAP}(10 \mathrm{mg}, 0.08$ mmol, 1 eq) and (Boc) $)_{2} \mathrm{O}\left(0.1 \mathrm{~mL}, 0.48 \mathrm{mmol}, 6\right.$ eq) was added, sequentially. TLC $\left(\mathrm{CH}_{3} \mathrm{OH} / \mathrm{CH}_{2} \mathrm{Cl}_{2}\right.$ 1:29) indicated consumption of the monoBoc ester $\left(\mathrm{R}_{f}=0.49,0.35\right)$ and formation of the two isomer products of diBoc ester $13\left(\mathrm{R}_{f}=0.87\right)$. After concentration under reduced pressure, EtOAc $(5 \mathrm{~mL})$ and $1 \mathrm{~N} \mathrm{HCl}$ (aq. 3 $\mathrm{mL}$ ) were used for partition. The organic layer was collected and dried over $\mathrm{Na}_{2} \mathrm{SO}_{4}$. After gravitational filtration, the filtrate was concentrated under reduced pressure to give a residue. Followed by flash chromatography with eluents EtOAc/n-hexane 1.5:8.5, a plastic-smell like yellow viscous liquid was 
obtained in $70 \%$ yield $(31 \mathrm{mg})$. The mixture could be further separated using HPLC with eluents of

EtOAc/ $n$-hexane 2.5:8.5 to give ortho-isomer, $\boldsymbol{o}-\mathbf{1 3} t_{R}=9.93 \mathrm{~min}$ and para-isomer, $\boldsymbol{p}-\mathbf{1 3} t_{R}=10.97 \mathrm{~min}$.

\section{Compound $o-13$}

${ }^{1} \mathrm{H}-\mathrm{NMR}\left(500 \mathrm{MHz}, \mathrm{CDCl}_{3}\right) \delta 1.53\left(\mathrm{~s}, 9 \mathrm{H}, \mathrm{CO}_{2} \mathrm{C}\left(\mathrm{CH}_{3}\right)_{3}\right), 1.65\left(\mathrm{~s}, 9 \mathrm{H}, \mathrm{CO}_{2} \mathrm{C}\left(\mathrm{CH}_{3}\right)_{3}\right), 3.11\left(\mathrm{dd}, J_{l a^{\prime}, 1 b^{\prime}}=15.0\right.$, $\left.J_{l a^{\prime} 2^{\prime}}=9.0 \mathrm{~Hz}, 1 \mathrm{H}, \mathrm{H}-1 \mathrm{a}^{\prime}\right), 3.38\left(\mathrm{dd}, J_{l b^{\prime} l a^{\prime}}=15.0, J_{l b^{\prime} 2^{\prime}}=4.0 \mathrm{~Hz}, 1 \mathrm{H}, \mathrm{H}-1 \mathrm{~b}^{\prime}\right), 3.71\left(\mathrm{~s}, 3 \mathrm{H}, \mathrm{CO}_{2} \mathrm{C}_{3}\right), 4.56$ $\left(\mathrm{ddd}, J_{2^{\prime}, 1 a^{\prime}}=9.0, J_{2^{\prime}, N H}=8.0, J_{2^{\prime}, 1 b^{\prime}}=4.0 \mathrm{~Hz}, 1 \mathrm{H}, \mathrm{H}-2^{\prime}\right), 5.03\left(\mathrm{~d}, J_{N H, 2^{\prime}}=8.0 \mathrm{~Hz}, 1 \mathrm{H}, \mathrm{NH}\right), 7.35(\mathrm{t}, J=8.0 \mathrm{~Hz}$, 1H, arom, H-6), 7.66 (s, 1H, arom, H-2), 7.88 (d, $J=7.5 \mathrm{~Hz}, 1 \mathrm{H}$, arom, H-5), 8.55 (d, $J=8.0 \mathrm{~Hz}, 1 \mathrm{H}$, arom, H-7). ${ }^{13} \mathrm{C}-\mathrm{NMR}\left(125 \mathrm{MHz}, \mathrm{CDCl}_{3}\right) \delta 28.09\left(\mathrm{CH}_{3}, \mathrm{CO}_{2} \mathrm{C}\left(\underline{\mathrm{CH}_{3}}\right)_{3}\right), 28.15\left(\mathrm{CH}_{3}, \mathrm{CO}_{2} \mathrm{C}\left(\underline{\mathrm{CH}}_{3}\right)_{3}\right), 29.68\left(\mathrm{CH}_{2}, \mathrm{C}-\right.$ 1'), $52.47\left(\mathrm{C}, \mathrm{CO}_{2} \underline{\mathrm{CH}} 3\right), 53.87\left(\mathrm{CH}, \mathrm{C}-2\right.$ '), $79.83\left(\mathrm{C}, \mathrm{CO}_{2} \underline{\mathrm{CCH}_{3}}\right), 85.02\left(\mathrm{C}, \mathrm{CO}_{2} \underline{\mathrm{CCH}_{3}}\right), 114.32(\mathrm{C}$, arom, C8), $119.92(\mathrm{CH}$, arom, C-7), 120.64 (CH, arom, C-2), 122.54 (C, arom, C-3), 123.49 (CH, arom, C-6),

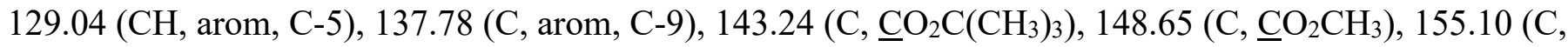
arom, C-4), $172.50\left(\mathrm{C}, \underline{\mathrm{CO}_{2}} \mathrm{CH}_{3}\right)$. Analysis for $\mathrm{C}_{22} \mathrm{H}_{29} \mathrm{~N}_{3} \mathrm{O}_{8}$ : calcd. $[\mathrm{M}+\mathrm{Na}]^{+}(\mathrm{m} / \mathrm{z})=486.1852(100.0 \%)$, $487.1886(23.8 \%), 488.1919(2.7 \%)$; calcd. $[2 \mathrm{M}+\mathrm{Na}]^{+}(\mathrm{m} / \mathrm{z})=949.3807(100.0 \%), 950.3841(47.6 \%)$, 951.3874 (11.1\%); ESI-Q-TOF HR-ESI-MS : found $[\mathrm{M}+\mathrm{Na}]^{+}=486.1852(100 \%), 487.1885(21.8 \%)$, $488.1903(3.6 \%) . \delta=0 \mathrm{ppm}$; found $[2 \mathrm{M}+\mathrm{Na}]^{+}=949.3794(7.3 \%), 950.3833(3.7 \%), 951.3843(1.2 \%) . \delta=-$ $1.3 \mathrm{ppm}$.

\section{Compound $p-13$}

${ }^{1} \mathrm{H}-\mathrm{NMR}\left(500 \mathrm{MHz}, \mathrm{CDCl}_{3}\right) \delta 1.40\left(\mathrm{~s}, 9 \mathrm{H}, \mathrm{CO}_{2} \mathrm{C}\left(\mathrm{C}_{3}\right)_{3}\right), 1.68\left(\mathrm{~s}, 9 \mathrm{H}, \mathrm{CO}_{2} \mathrm{C}\left(\mathrm{C}_{3}\right)_{3}\right), 3.16\left(\mathrm{dd}, J_{1 a^{\prime}, 1 b^{\prime}}=14.5\right.$, $\left.J_{1 a^{\prime} 2^{\prime}}=5.0 \mathrm{~Hz}, 1 \mathrm{H}, \mathrm{H}-1 \mathrm{a}^{\prime}\right), 3.30\left(\mathrm{dd}, J_{1 b^{\prime}, 1 a^{\prime}}=14.5, J_{1 b^{\prime}, 2^{\prime}}=5.0 \mathrm{~Hz}, 1 \mathrm{H}, \mathrm{H}-1 \mathrm{~b}^{\prime}\right), 3.69\left(\mathrm{~s}, 3 \mathrm{H}, \mathrm{CO}_{2} \mathrm{C}_{3}\right), 4.62$ $\left(\mathrm{ddd}, J_{2^{\prime}, N H}=7.0, J_{2^{\prime}, 1 a^{\prime}}=5.0, J_{2^{\prime}, 1 b^{\prime}}=5.0 \mathrm{~Hz}, 1 \mathrm{H}, \mathrm{H}-2{ }^{\prime}\right), 5.13\left(\mathrm{~d}, J_{N H, 2^{\prime}}=7.0 \mathrm{~Hz}, 1 \mathrm{H}, \mathrm{NH}\right), 7.56(\mathrm{~d}, J=8.5 \mathrm{~Hz}$, 
1H, arom, H-4), 7.63 (s, 1H, arom, H-2), 8.12 (dd,1H, J=8.5, 2.0 Hz, arom, H-5), 9.03 (bs, 1H, arom, H-7).

${ }^{13} \mathrm{C}-\mathrm{NMR}\left(125 \mathrm{MHz}, \mathrm{CDCl}_{3}\right) \delta 27.78\left(\mathrm{CH}_{2}, \mathrm{C}-1^{\prime}\right), 28.09\left(\mathrm{CH}_{3}, \mathrm{CO}_{2} \mathrm{C}\left(\underline{\mathrm{C}} \mathrm{H}_{3}\right)_{3}\right), 28.26\left(\mathrm{CH}_{3}, \mathrm{CO}_{2} \mathrm{C}\left(\underline{\mathrm{CH}_{3}}\right)_{3}\right)$,

$52.51\left(\mathrm{C}, \mathrm{CO}_{2} \underline{\mathrm{CH}}_{3}\right), 53.61\left(\mathrm{CH}, \mathrm{C}-2^{\prime}\right), 80.22\left(\mathrm{C}, \mathrm{CO}_{2} \underline{\mathrm{CCH}_{3}}\right), 85.30\left(\mathrm{C}, \mathrm{CO}_{2} \underline{\mathrm{CCH}}_{3}\right), 111.93(\mathrm{CH}$, arom, $\mathrm{C}-7)$,

115.34 (C, arom, C-3), $117.85(\mathrm{CH}$, arom, C-2), $119.01(\mathrm{CH}$, arom, C-5), 129.07 (CH, arom, C-4), 134.10

(C, arom, C-9), 135.32 (C, arom, C-8), $145.14\left(\mathrm{C}, \underline{\mathrm{CO}} 2 \mathrm{C}\left(\mathrm{CH}_{3}\right)_{3}\right), 148.65\left(\mathrm{C}, \underline{\mathrm{CO}}_{2} \mathrm{C}\left(\mathrm{CH}_{3}\right)_{3}\right), 154.98(\mathrm{C}$, arom,

C-6), $171.91\left(\mathrm{C}, \underline{\mathrm{CO}}_{2} \mathrm{CH}_{3}\right)$. Analysis for $\mathrm{C}_{22} \mathrm{H}_{29} \mathrm{~N}_{3} \mathrm{O}_{8}$ : calcd. $[\mathrm{M}+\mathrm{Na}]^{+}(\mathrm{m} / \mathrm{z})=486.1852(100.0 \%), 487.1886$

(23.8\%), $488.1919(2.7 \%) ;$ calcd. $[2 \mathrm{M}+\mathrm{Na}]^{+}(\mathrm{m} / \mathrm{z})=949.3807(100.0 \%), 950.3841(47.6 \%), 951.3874$

(11.1\%); ESI-Q-TOF HR-ESI-MS: found $[\mathrm{M}+\mathrm{Na}]^{+}=486.1850(31.4 \%), 487.1881(6.8 \%), 488.1905(1.2 \%)$

$\delta=-0.4 \mathrm{ppm} ;$ Found $[2 \mathrm{M}+\mathrm{Na}]^{+}=949.3752(100 \%), 950.3786(46.2 \%), 951.3811(12.1 \%) \delta=-5.8 \mathrm{ppm}$

1,3-(2-N,N)triBoc-6-nitro tryptophan methyl ester $\boldsymbol{p}-14$ and 1,3-(2-N,N)triBoc-4-nitro tryptophan methyl ester $\boldsymbol{o - 1 4}$

To a mixture of the above nitro tryptophan $12(11.2 \mathrm{~g}, 44 \mathrm{mmol})$ in $\mathrm{CH}_{3} \mathrm{OH}(200 \mathrm{~mL})$ at ice bath under $\mathrm{N}_{2}$ was added $\mathrm{SOCl}_{2}(19 \mathrm{~mL}, 264 \mathrm{mmol}, 6 \mathrm{eq})$ within $6 \mathrm{~min}$. The ice bath was removed and the mixture was refluxed at $85^{\circ} \mathrm{C}$. TLC $\left(\mathrm{CH}_{3} \mathrm{OH} / \mathrm{CH}_{2} \mathrm{Cl}_{2} 6: 4\right)$ indicated consumption of starting material $12\left(\mathrm{R}_{f}=0.19\right)$ and formation of the methy ester tryptophan $\left(\mathrm{R}_{f}=0.95\right)$. After $40 \mathrm{~min}$, the mixture was concentrated under reduced pressure at $45{ }^{\circ} \mathrm{C}$ followed by distillation with $\mathrm{CH}_{3} \mathrm{OH}(40 \mathrm{~mL})$ and $\mathrm{Et}_{3} \mathrm{~N}(10 \mathrm{~mL}) \times 2$. A fresh distilled $\mathrm{CH}_{3} \mathrm{OH}(40 \mathrm{~mL})$ was added followed by addition of $(\mathrm{Boc})_{2} \mathrm{O}(13.78 \mathrm{~g}, 66 \mathrm{mmol}, 1.5 \mathrm{eq}), \mathrm{Et} 3 \mathrm{~N}$ (64.66 g, $616 \mathrm{mmol}, 14 \mathrm{eq})$ and $\mathrm{CH}_{3} \mathrm{OH}(50 \mathrm{~mL})$, sequentially. The total mixture comprised about a volume of $150 \mathrm{~mL}$. The mixture was stirred at $\mathrm{rt}$ for $20 \mathrm{~min}$ and the TLC $\left(\mathrm{CH}_{3} \mathrm{OH} / \mathrm{CH}_{2} \mathrm{Cl}_{2}\right.$ 1:19) indicated consumption of the methyl ester tryptophan $\left(\mathrm{R}_{f}=0.25\right)$ and formation of the monoBoc-protected ester 
intermediate $\left(\mathrm{R}_{f}=0.81\right.$ and 0.91$)$. The mixture was then concentrated under reduced pressure. After flash chromatography $\left(\mathrm{CH}_{3} \mathrm{OH} / \mathrm{CH}_{2} \mathrm{Cl}_{2}\right.$ 1:49), the fractions were concentrated to give a dark red viscous liquid (9.38 g, $25 \mathrm{mmol})$. Followed by addition of fresh distilled $\mathrm{CH}_{3} \mathrm{CN}(80 \mathrm{~mL})$, a solution of DMAP $(3.0 \mathrm{~g}, 25$ mmol, 1 eq) in $\mathrm{CH}_{3} \mathrm{CN}(20 \mathrm{~mL})$ and a solution of $(\mathrm{Boc})_{2} \mathrm{O}(10.9 \mathrm{~g}, 50 \mathrm{mmol}, 2 \mathrm{eq})$ in $\mathrm{CH}_{3} \mathrm{CN}(10 \mathrm{~mL})$, sequentially, the mixture was stirred under Ar at rt for $4 \mathrm{~h}$. TLC (EtOAc/n-hexane 2:8) indicated consumption of monoBoc tryptophan $\left(\mathrm{R}_{f}=0.10\right)$ and formation of the triBoc-protected product $14\left(\mathrm{R}_{f}=\right.$ $0.52,0.43)$. The mixture was concentrated under reduced pressure and the residue obtained was chromatographed over $\mathrm{SiO}_{2}$ with EtOAc/n-hexane 1.5:8.5 to give a yellow viscous liquid in $30 \%$ yield (7.6 g). A catalytic amount of sample was further purified using HPLC with EtOAc/ $n$-hexane 1:7 to give the ortho-isomer $\boldsymbol{o}-14, t_{R}=24.33 \mathrm{~min}$ and para-isomer $\boldsymbol{p}-14, t_{R}=26.07 \mathrm{~min}$.

\section{Compound $p$-14}

${ }^{1} \mathrm{H}-\mathrm{NMR}\left(500 \mathrm{~Hz}, \mathrm{CDCl}_{3}\right) \delta 1.27\left(\mathrm{~s}, 9 \mathrm{H}, \mathrm{CO}_{2} \mathrm{C}\left(\mathrm{C}_{3}\right)_{3}\right), 1.29\left(\mathrm{~s}, 9 \mathrm{H}, \mathrm{CO}_{2} \mathrm{C}\left(\mathrm{C}_{3}\right)_{3}\right), 1.62(\mathrm{~s}, 9 \mathrm{H}$, $\left.\mathrm{CO}_{2} \mathrm{C}\left(\underline{\mathrm{C}}_{3}\right)_{3}\right), 3.30\left(\mathrm{dd}, J_{1 a^{\prime}, 1 b^{\prime}}=15.0, J_{1 a^{\prime} 2^{\prime}}=10.0 \mathrm{~Hz}, 1 \mathrm{H}, \mathrm{H}-1 \mathrm{a}\right), 3.47\left(\mathrm{dd}, J_{1 b^{\prime}, 1^{\prime} a}=15.0, J_{1 b^{\prime}, 2^{\prime}}=5.0 \mathrm{~Hz}\right.$, $\left.1 \mathrm{H}, \mathrm{H}-1 \mathrm{~b}^{\prime}\right), 3.70\left(\mathrm{~s}, 3 \mathrm{H}, \mathrm{CO}_{2} \underline{\mathrm{C}}_{3}\right), 5.12\left(\mathrm{dd}, J_{2^{\prime}, 1 a^{\prime}}=10.0, J_{2^{\prime}, 1 b^{\prime}}=5.0 \mathrm{~Hz}, 1 \mathrm{H}, \mathrm{H}-2^{\prime}\right), 7.57(\mathrm{~d}, J=8.5 \mathrm{~Hz}, 1 \mathrm{H}$, arom), 7.61 (s, 1H, arom), 8.07 (dd, $J=8.5 \mathrm{~Hz}, 2.0 \mathrm{~Hz}, 1 \mathrm{H}$, arom), 8.99 (s, 1H, arom). ${ }^{13} \mathrm{C}-\mathrm{NMR}(125$ $\left.\mathrm{MHz}, \mathrm{CDCl}_{3}\right) \delta 25.42\left(\mathrm{CH}_{2}, \mathrm{C}-1^{\prime}\right), 27.77\left(\mathrm{CH}_{3}, \mathrm{CO}_{2} \mathrm{C}\left(\underline{\mathrm{C}} \mathrm{H}_{3}\right)_{3}\right), 28.08\left(\mathrm{CH}_{3}, \mathrm{CO}_{2} \mathrm{C}\left(\underline{\mathrm{CH}}_{3}\right)_{3}\right), 52.44\left(\mathrm{CH}_{3}\right.$, $\left.\mathrm{CO}_{2} \underline{\mathrm{CH}_{3}}\right), 57.98\left(\mathrm{CH}, \mathrm{C}-2^{\prime}\right), 83.29\left(\mathrm{C}, \mathrm{CO}_{2} \underline{\mathrm{C}}(\mathrm{CH})_{3}\right), 85.08\left(\mathrm{C}, \mathrm{CO}_{2} \underline{\mathrm{C}}(\mathrm{CH})_{3}\right), 111.77(\mathrm{CH}$, arom $), 116.70(\mathrm{C}$, arom), $117.86(\mathrm{CH}$, arom), $119.12(\mathrm{CH}$, arom $), 129.38(\mathrm{CH}$, arom $), 134.18(\mathrm{C}$, arom $), 135.34(\mathrm{C}$, arom), $145.04\left(\mathrm{C}, \underline{\mathrm{CO}}_{2} \mathrm{C}\left(\mathrm{CH}_{3}\right)_{3}\right), 148.67\left(\mathrm{C}, \underline{\mathrm{CO}}_{2} \mathrm{CH}_{3}\right), 151.94(\mathrm{C}$, arom $), 170.47\left(\mathrm{C}, \underline{\mathrm{CO}}_{2} \mathrm{CH}_{3}\right)$. Analysis for $\mathrm{C}_{27} \mathrm{H}_{37} \mathrm{~N}_{3} \mathrm{O}_{10}$ : calcd. [M+Na] $]^{+}(\mathrm{m} / \mathrm{z})=586.2377$ (100.0\%), 587.2410 (29.2\%), 588.2444 (4.1\%); calcd. 
$[2 \mathrm{M}+\mathrm{Na}]^{+}=1149.4856(100.0 \%), 1150.4889(58.4 \%), 1151.4923$ (16.7\%); ESI-Q-TOF HR-ESI-MS: Found $[\mathrm{M}+\mathrm{Na}]^{+}=586.2365(72 \%), 587.2400(18.5 \%), 588.2423(3 \%) . \delta=-2.0 \mathrm{ppm} ;$ found $[2 \mathrm{M}+\mathrm{Na}]^{+}=$ $1149.4826(100.0 \%), 1150.4858(54.3 \%), 1151.4888(17.3 \%) . \delta=-2.6 \mathrm{ppm}$.

\section{Compound $o-\mathbf{1 4}^{10}$}

${ }^{1} \mathrm{H}-\mathrm{NMR}\left(500 \mathrm{~Hz}, \mathrm{CDCl}_{3}\right) \delta 1.23\left(\mathrm{~s}, 18 \mathrm{H}, \mathrm{CO}_{2} \mathrm{C}\left(\mathrm{C}_{3}\right)_{3}\right), 1.58\left(\mathrm{~s}, 9 \mathrm{H}, \mathrm{CO}_{2} \mathrm{C}\left(\mathrm{C}_{3}\right)_{3}\right), 3.30\left(\mathrm{dd}, J_{1 a^{\prime}, 1 b^{\prime}}=15.0\right.$, $\left.J_{1 a^{\prime} 2^{\prime}}=11.0 \mathrm{~Hz}, 1 \mathrm{H}, \mathrm{H}-1^{\prime} \mathrm{a}\right), 3.71\left(\mathrm{~s}, 3 \mathrm{H}, \mathrm{CO}_{2} \mathrm{C}_{3}\right), 3.72\left(\mathrm{dd}, J_{1 b^{\prime}, 1 a^{\prime}}=15.0, J_{1 b^{\prime}, 2^{\prime}}=4.5 \mathrm{~Hz}, 1 \mathrm{H}, \mathrm{H}-1 \mathrm{~b}^{\prime}\right), 5.22$ $\left(\mathrm{dd}, J_{2^{\prime}, 1 a^{\prime}}=11.0, J_{2^{\prime}, 1 b^{\prime}}=4.5 \mathrm{~Hz}, 1 \mathrm{H}, \mathrm{H}-2^{\prime}\right), 7.30(\mathrm{t}, J=8.0 \mathrm{~Hz}, 1 \mathrm{H}$, arom), 7.47 (s, 1H, arom), $7.90(\mathrm{dd}, J=$ $8.0 \mathrm{~Hz}, 1.0 \mathrm{~Hz}, 1 \mathrm{H}$, arom), $8.51\left(\mathrm{~d}, J=8.0 \mathrm{~Hz}, 1 \mathrm{H}\right.$, arom). ${ }^{13} \mathrm{C} \mathrm{NMR}\left(125 \mathrm{MHz}, \mathrm{CDCl}_{3}\right) \delta 27.72\left(\mathrm{CH}_{3}\right.$, $\left.\mathrm{CO}_{2} \mathrm{C}\left(\underline{\mathrm{CH}}_{3}\right)_{3}\right), 27.93\left(\mathrm{CH}_{2}, \mathrm{C}-1^{\prime}\right), 28.05\left(\mathrm{CH}_{3}, \mathrm{CO}_{2} \mathrm{C}\left(\underline{\mathrm{C}} \mathrm{H}_{3}\right)_{3}\right), 52.30\left(\mathrm{CH}_{3}, \mathrm{CO}_{2} \underline{\mathrm{CH}}_{3}\right), 58.31\left(\mathrm{CH}, \mathrm{C}-2^{\prime}\right), 82.81$

$\left(\mathrm{C}, \mathrm{CO}_{2} \underline{\mathrm{C}}(\mathrm{CH})_{3}\right), 84.91\left(\mathrm{C}, \mathrm{CO}_{2} \underline{\mathrm{C}}(\mathrm{CH})_{3}\right), 114.94(\mathrm{C}$, arom), $120.05(\mathrm{C}$, arom $), 120.74(\mathrm{CH}$, arom $), 122.68$ (C, arom), $\left.123.45(\mathrm{CH}, \operatorname{arom}), 129.98(\mathrm{CH}, \operatorname{arom}), 137.95(\mathrm{C}, \operatorname{arom}), 143.23\left(\mathrm{C}, \underline{\mathrm{CO}}_{2} \mathrm{C}_{\left(\mathrm{CH}_{3}\right)}\right)_{3}\right), 148.61(\mathrm{C}$, $\left.\underline{\mathrm{CO}}_{2} \mathrm{CH}_{3}\right), 151.78\left(\mathrm{C}\right.$, arom), $170.56\left(\mathrm{C}, \underline{\mathrm{CO}}_{2} \mathrm{CH}_{3}\right)$. Analysis for $\mathrm{C}_{27} \mathrm{H}_{37} \mathrm{~N}_{3} \mathrm{O}_{10}$ : calcd. $[\mathrm{M}+\mathrm{Na}]^{+}(\mathrm{m} / \mathrm{z})=$ $586.2377(100.0 \%), 587.2410(29.2 \%), 588.2444(4.1 \%) ;$ calcd. $[2 \mathrm{M}+\mathrm{Na}]^{+}=1149.4856(100.0 \%)$ 1150.4889 (58.4\%), 1151.4923 (16.7\%); ESI-Q-TOF HR-ESI-MS: found $[\mathrm{M}+\mathrm{Na}]^{+}=586.2372(100 \%)$, $587.2408(27.5 \%), 588.2434(4 \%) . \delta=-0.85 \mathrm{ppm}$; found $[2 \mathrm{M}+\mathrm{Na}]^{+}=1149.4851(24 \%), 1150.4883(13 \%)$, $1151.4915(5 \%) . \delta=-0.43 \mathrm{ppm}$.

1,3-(2-N,N)triBoc-6-amino tryptophan methyl ester $\boldsymbol{p}$-15 and 1,3-(2-N,N)triBoc-4-amino tryptophan methyl ester $\boldsymbol{o - 1 5}$

A mixture of the triboc tryptophan $14(7.6 \mathrm{~g}, 14 \mathrm{mmol})$ in $\mathrm{CH}_{2} \mathrm{Cl}_{2}(100 \mathrm{~mL})$ was stirred in a roundbottomed flask $(500 \mathrm{~mL})$ under $\mathrm{N}_{2}$ with a cooling of an ice bath. Zink dust (grain size of 1-2 mm, $45.7 \mathrm{~g}$, 
$700 \mathrm{mmol}, 50 \mathrm{eq})$ was added. A portion $(32 \mathrm{~mL})$ of a mixture of $\mathrm{AcOH}(36 \mathrm{~mL})$ in $\mathrm{CH}_{2} \mathrm{Cl}_{2}(74 \mathrm{~mL})$ was

added within $10 \mathrm{~min}$. Removing the ice bath, the mixture turned from yellow to pare green after $10 \mathrm{~min}$. It changed to gray green after $30 \mathrm{~min}$. TLC (EtOAc/n-hexane 2:8) indicated consumption of the starting material $14\left(\mathrm{R}_{f}=0.57\right)$ and formation of two constitutional isomers $\boldsymbol{o}-15$ and $p$-15 $\left(\mathrm{R}_{f}=0.26,0.13\right)$. After gravitational filtration, the filtrates were partitioned between $\mathrm{CH}_{2} \mathrm{Cl}_{2}(50 \mathrm{~mL})$ and satd. $\mathrm{NaHCO}_{3}$ (aq., 30 $\mathrm{mL}) . \mathrm{NaHCO}_{3}$ was added slowly until no bubbling evolved $(\mathrm{pH} \sim 9)$. The organic layer was collected and dried using $\mathrm{Mg}_{2} \mathrm{SO}_{4}$ followed by gravitational filtration. The filtrate was concentrated under reduced pressure to give an orange-yellow viscous liquid in $92 \%$ yield $(6.7 \mathrm{~g})$.

1,3-(2- $N, N)$ triBoc-6-iodo tryptophan methyl ester $\boldsymbol{p}-\mathbf{1 6}$ and 1,3-(2- $N, N)$ triBoc-4-iodo tryptophan methyl ester $\boldsymbol{o - 1 6}$

To a round-bottomed flask $(500 \mathrm{~mL})$ containing the above intermediate $\mathbf{1 5}(5.1 \mathrm{~g}, 9.5 \mathrm{mmol})$ was added THF (40 mL), $\mathrm{H}_{2} \mathrm{O}(10 \mathrm{~mL})$ and a solution of $5 \% \mathrm{HCl}(30 \mathrm{~g}, 4 \mathrm{eq})$, diluted from $\mathrm{HCl}(37 \%$ wt., $15 \mathrm{~g})$ to 100 g with $\mathrm{H}_{2} \mathrm{O}$, sequentially. The mixture was then cooled to $0{ }^{\circ} \mathrm{C}$ by an ice bath. To the mixture was added a mixed solution of aqueous $\mathrm{NaNO}_{2}\left(5 \mathrm{~mL}, 10.4 \mathrm{mmol}, 1.1\right.$ eq.), prepared from $1.5 \mathrm{~g} \mathrm{NaNO}_{2}$ in $\mathrm{H}_{2} \mathrm{O}(10 \mathrm{~mL})$, within $3 \mathrm{~min}$. The mixture turned from dark orange to dark brown. After a further stirring for 8 min, the whole mixture was poured into a round-bottomed flask $(100 \mathrm{~mL})$ containing KI $(9.77 \mathrm{~g}, 58.9 \mathrm{mmol}, 6.2 \mathrm{eq})$, $\mathrm{I}_{2}(2.4 \mathrm{~g}, 9.5 \mathrm{mmol}, 1 \mathrm{eq})$ and $\mathrm{H}_{2} \mathrm{O}(40 \mathrm{~mL})$. After stirring at rt for $10 \mathrm{~min}$, TLC (EtOAc/n-hexane 3:7) indicated consumption of starting material $15\left(\mathrm{R}_{f}=0.41\right)$ and formation of the product $16\left(\mathrm{R}_{f}=0.68\right)$. The mixture was than treated with $\mathrm{NaHCO}_{3}$ until $\mathrm{pH}$ reached $9(\sim 10 \mathrm{~mL})$. The mixture was then partitioned between EtOAc $(250 \mathrm{~mL})$ and $\mathrm{H}_{2} \mathrm{O}(100 \mathrm{~mL})$. After washing with $\mathrm{H}_{2} \mathrm{O}(100 \mathrm{~mL})$ and back extraction with 
$\mathrm{CH}_{2} \mathrm{Cl}_{2}$ twice $(50 \mathrm{~mL} \times 1$ and $10 \mathrm{~mL} \times 1)$, the organic layers combined were reduced with saturated $\mathrm{Na}_{2} \mathrm{~S}_{2} \mathrm{O}_{3}$ (aq., $60 \mathrm{~mL} \times 2$ ). The organic layer was collected, dried with $\mathrm{Mg}_{2} \mathrm{SO}_{4}$ and filtered through gravitational filtration. The filtrate was concentrated under reduced pressure to give a residue. After flash chromatography, a yellow oil was obtained in $51 \%$ yield $(3.1 \mathrm{~g})$ over the two steps. Because the mixture cannot be purified using HPLC (see sup. Inf. Fig. 15), the spectroscopic analysis of pure compound will be provided after borylation.

${ }^{1} \mathrm{H}-\mathrm{NMR}\left(500 \mathrm{MHz}, \mathrm{CDCl}_{3}\right) \delta 1.31\left(\mathrm{~s}, 18 \mathrm{H}, \mathrm{CO}_{2} \mathrm{C}\left(\underline{\mathrm{CH}}_{3}\right)_{3}, \boldsymbol{p}\right.$-16 + o-16 + deiodo $), 1.61(\mathrm{~s}, 11 \mathrm{H}$, $\mathrm{CO}_{2} \mathrm{C}\left(\underline{\mathrm{CH}}_{3}\right)_{3}, \boldsymbol{p}-\mathbf{- 1 6}+\boldsymbol{o}-16+$ deiodo $), 3.16\left(\mathrm{dd}, J_{1 a^{\prime}, 1 b^{\prime}}=14.5, J_{1 a^{\prime}, 2^{\prime}}=10.0 \mathrm{~Hz}, 1 \mathrm{H}, \mathrm{H}-1 \mathrm{a}^{\prime}\right.$, deiodo $), 3.31(\mathrm{dd}$, $\left.J_{l a^{\prime}, 1 b^{\prime}}=15.0, \mathrm{~J}_{1 \mathrm{a}^{\prime} 2^{\prime}}=10.0 \mathrm{~Hz}, 1 \mathrm{H}, \mathrm{H}-1 \mathrm{a}^{\prime}, \boldsymbol{p}-\mathbf{6}+\boldsymbol{o}-\mathbf{6}\right), 3.45\left(\mathrm{dd}, J_{1 b^{\prime}, 1 a^{\prime}}=15.0, J_{1 b^{\prime}, 2^{\prime}}=5.0 \mathrm{~Hz}, 1 \mathrm{H}, \mathrm{H}_{1 b^{\prime}}, \boldsymbol{p}-16+\right.$ o-16), $3.74\left(\mathrm{~s}, 3 \mathrm{H}, \mathrm{CO}_{2} \underline{\mathrm{H}}_{3}, \boldsymbol{p}-\mathbf{1 6}+\boldsymbol{o}-\mathbf{- 1 6}+\right.$ deiodo), $5.15\left(\mathrm{dd}, J_{2^{\prime}, 1 a^{\prime}}=10.0, J_{2^{\prime}, 1 b^{\prime}}=5.0 \mathrm{~Hz}, 1 \mathrm{H}, \mathrm{H}_{2^{\prime}}, \boldsymbol{p}-\mathbf{1 6}\right)$, $5.18\left(\mathrm{dd}, J_{2^{\prime}, 1 a^{\prime}}=10.0, J_{2^{\prime}, 1 b^{\prime}}=5.0 \mathrm{~Hz}, 1 \mathrm{H}, \mathrm{H}-2^{\prime}, \boldsymbol{o}-16\right), 5.44\left(\mathrm{dd}, J_{2^{\prime}, 1 a^{\prime}}=12.0, J_{2^{\prime}, 1 b^{\prime}}=4.5 \mathrm{~Hz}, 1 \mathrm{H}, \mathrm{H}-2^{\prime}\right.$, deiodo), $6.92(\mathrm{t}, J=5.0 \mathrm{~Hz}, 1 \mathrm{H}$, arom, deiodo), $7.19(\mathrm{t}, 2 \mathrm{H}$, arom, deiodo), $7.25(\mathrm{~d}, J=8.5 \mathrm{~Hz}, 1 \mathrm{H}$, arom, p16), $7.29(\mathrm{~s}, 1 \mathrm{H}$, arom, p-16), $7.35(\mathrm{~d}, 2 \mathrm{H}$, arom, o-16 + deiodo), $7.50(\mathrm{~d}, J=8.5 \mathrm{~Hz}, 1 \mathrm{H}$, arom, H-5, p-16 + o-16), 8.09 (d, $J=7.0 \mathrm{~Hz}, 1 \mathrm{H}$, arom, deiodo), 8.19 (d, $J=8.5 \mathrm{~Hz}, 1 \mathrm{H}$, arom, o-16), 8.52 (bs, 1H, arom, H-7, p-16). Ratio of p-16 vs. o-16 was 5:1. ${ }^{13} \mathrm{C}-\mathrm{NMR}\left(125 \mathrm{MHz}, \mathrm{CDCl}_{3}\right) \delta 25.40\left(\mathrm{CH}_{2}, \mathrm{C}-1^{\prime}\right), 27.72\left(\mathrm{CH}_{3}\right.$, $\left.\mathrm{CO}_{2} \mathrm{C}\left(\underline{\mathrm{CH}}_{3}\right)_{3}\right), 28.07\left(\mathrm{CH}_{3}, \mathrm{CO}_{2} \mathrm{C}\left(\underline{\mathrm{CH}}_{3}\right)_{3}\right), 52.32\left(\mathrm{CH}_{3}, \mathrm{CO}_{2} \underline{\mathrm{CH}}_{3}\right), 58.10\left(\mathrm{CH}, \mathrm{C}-2^{\prime}\right), 83.05\left(\mathrm{C}, \mathrm{CO}_{2} \underline{\mathrm{C}}(\mathrm{CH})_{3}\right)$, $83.95\left(\mathrm{C}, \mathrm{CO}_{2} \underline{\mathrm{C}}(\mathrm{CH})_{3}\right), 88.71\left(\mathrm{C}, \mathrm{CO}_{2} \underline{\mathrm{C}}(\mathrm{CH})_{3}\right), 116.43(\mathrm{CH}$, arom $), 120.49(\mathrm{CH}$, arom $), 124.21(\mathrm{CH}$, arom $)$,

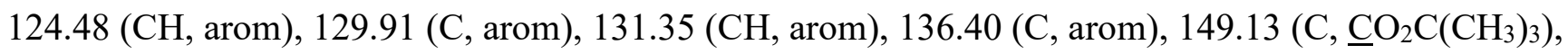
$151.83\left(\mathrm{C}, \underline{\mathrm{CO}_{2}} \mathrm{CH}_{3}\right), 170.64\left(\mathrm{C}, \underline{\mathrm{CO}}_{2} \mathrm{CH}_{3}\right)$. Analysis for $\mathrm{C}_{27} \mathrm{H}_{37} \mathrm{IN}_{2} \mathrm{O}_{8}$ : calcd. $[\mathrm{M}+\mathrm{Na}]^{+}(\mathrm{m} / \mathrm{z})=667.1492$ $(100.0 \%), 668.1526(29.2 \%), 669.1559(4.1 \%) ;$ calcd. $[2 \mathrm{M}+\mathrm{Na}]^{+}(\mathrm{m} / \mathrm{z})=1311.3087(100.0 \%), 1312.3120$ 
(58.4\%), 1313.3154 (16.7\%); ESI-Q-TOF HR-ESI-MS: found $[\mathrm{M}+\mathrm{Na}]^{+}=667.1480(100 \%), 668.1515(25.0$

$\%), 669.1538(4.0 \%) . \delta=-1.8 \mathrm{ppm}$; found $[2 \mathrm{M}+\mathrm{Na}]^{+}=1311.3067(15.6 \%), 1312.3102(9 \%), 1313.3138$

$(2.9 \%) . \delta=-1.5 \mathrm{ppm}$.

1,3-(2-N,N)triBoc-6-boronopinacol tryptophan methyl ester (TB-6-BT) 5

A two-necked round-bottomed flask $(50 \mathrm{~mL})$ containing KOAC (549 mg, $4.4 \mathrm{mmol}, 4.0 \mathrm{eq})$ was dried in an oven at $120{ }^{\circ} \mathrm{C}$ for over $96 \mathrm{~h}$. A second flask (50 mL) containing iodo compounds $\mathbf{1 6}(710 \mathrm{mg}, 1.1 \mathrm{mmol})$ and boronopinacol ( $82 \mathrm{mg}, 2.2 \mathrm{mmol}, 2 \mathrm{eq})$ was dried twice by evaporation with toluene at $50{ }^{\circ} \mathrm{C}$ under reduced pressure. The third spin-like flask $(50 \mathrm{~mL})$ containing a complex of dichloro-[1,1'bis(diphenylphosphino)ferrocene] palladium (II) $\left[\mathrm{PdCl}_{2}(\mathrm{dppf})\right](10 \mathrm{mg}, 0.015 \mathrm{mmol}, 0.1 \mathrm{eq})$ was dried twice with toluene by coevaporation at $55^{\circ} \mathrm{C}$ under reduced pressure. A flask containing dried DMSO (over $4 \AA$ MS for $48 \mathrm{~h}$ ) was bubbled with $\mathrm{N}_{2}$ for $15 \mathrm{~min}$. To the two-necked round bottomed flask containing KOAc, a mixture of iodo tryptophan compound and boronpinacol in DMSO $(12 \mathrm{~mL})$ and a solution of $\mathrm{PdCl}_{2}(\mathrm{dppf})$ in $\mathrm{DMSO}$ $(4 \mathrm{~mL})$ were sequentially added under sufficient stirring. The mixture was moved to an oil bath that has been preheated to $90{ }^{\circ} \mathrm{C}$ and the stirring was continued through bubbling. The mixture turned from light orange to dark brown after $10 \mathrm{~min}$. TLC (EtOAc/n-hexane 2:8) indicated the consumption of the starting material $16\left(\mathrm{R}_{f}\right.$ $=0.46)$ and formation of the product $5\left(\mathrm{R}_{f}=0.52\right)$. The reaction was terminated post $1 \mathrm{~h}$ of reaction by partitioning between $\mathrm{CH}_{2} \mathrm{Cl}_{2}(40 \mathrm{~mL})$ and $\mathrm{HCl}$ (aq. $0.2 \mathrm{~N}, 20 \mathrm{~mL}$ ). The organic layer was dried using $\mathrm{Mg}_{2} \mathrm{SO}_{4}$ followed by filtration using a celite pad and concentration under reduced pressure. The residue contained a significant amount of DMSO that can be further reduced by a second extraction or purified through flash chromatography using the gradient mode of EtOAc/n-hexane 1:19 $\rightarrow$ 1:9 to give a pleasant Hinoki-essential 
oil-odor pale yellow viscous gum in a $63 \%$ yield $(449 \mathrm{mg})$. A corrected yield based on the recovered iodo tryptophan 16 was $79 \%$. Note: about $25 \%$ was the deiodotryptophan analog. ${ }^{1} \mathrm{H}-\mathrm{NMR}\left(500 \mathrm{MHz}, \mathrm{CDCl}_{3}\right)^{1} \delta$ $1.30\left(\mathrm{~s}, 18 \mathrm{H}, \mathrm{CO}_{2} \mathrm{C}\left(\underline{\mathrm{C}}_{3}\right)_{3}\right), 1.32(\mathrm{~s}, 12 \mathrm{H}$, pinacol$), 1.62\left(\mathrm{~s}, 9 \mathrm{H}, \mathrm{CO}_{2} \mathrm{C}\left(\mathrm{C}_{3}\right)_{3}\right), 3.34\left(\mathrm{dd}, J_{1 a^{\prime}, 1 b^{\prime}}=14.5, J_{1 a^{\prime}, 2^{\prime}}=\right.$ $\left.10.0 \mathrm{~Hz}, 1 \mathrm{H}, \mathrm{H}-1 \mathrm{a}^{\prime}\right), 3.50\left(\mathrm{dd}, J_{1 b^{\prime}, 1 a^{\prime}}=15.0, J_{1 b^{\prime}, 2^{\prime}}=4.5 \mathrm{~Hz}, 1 \mathrm{H}, \mathrm{H}-1 \mathrm{~b}^{\prime}\right), 3.74\left(\mathrm{~s}, 3 \mathrm{H}, \mathrm{CO}_{2} \mathrm{C}_{3}\right), 5.18\left(\mathrm{dd}, J_{2^{\prime}, 1 a^{\prime}}\right.$ $\left.=10.0, J_{2^{\prime}, 1 b^{\prime}}=4.5 \mathrm{~Hz}, 1 \mathrm{H}, \mathrm{H}-2\right), 7.40(\mathrm{~s}, 1 \mathrm{H}$, arom, H-2), $7.49(\mathrm{~d}, J=8.0 \mathrm{~Hz}, 1 \mathrm{H}$, arom, H-4), $7.65(\mathrm{~d}, J=$ $8.0 \mathrm{~Hz}, 1 \mathrm{H}$, arom, H-5) 8.59 (s,1H, arom, H-7). ${ }^{13} \mathrm{C}-\mathrm{NMR}\left(125 \mathrm{MHz}, \mathrm{CDCl}_{3}\right) \delta 24.86\left(\mathrm{CH}_{3}\right.$, pinacol), 25.50 $\left(\mathrm{CH}_{2}, \mathrm{C}-1^{\prime}\right), 27.72\left(\mathrm{CH}_{3}, \mathrm{CO}_{2} \mathrm{C}\left(\underline{\mathrm{CH}_{3}}\right)_{3}\right), 28.10\left(\mathrm{CH}_{3}, \mathrm{CO}_{2} \mathrm{C}\left(\underline{\mathrm{CH}}_{3}\right)_{3}\right), 52.30\left(\mathrm{CH}_{3}, \mathrm{CO}_{2} \underline{\mathrm{CH}}_{3}\right), 58.16\left(\mathrm{CH}, \mathrm{C}-2^{\prime}\right)$, $82.96\left(\mathrm{C}, \mathrm{CO}_{2} \underline{\mathrm{C}}(\mathrm{CH})_{3}\right), 83.32\left(\mathrm{C}, \mathrm{CO}_{2} \underline{\mathrm{C}}(\mathrm{CH})_{3}\right), 83.58\left(\mathrm{C}, \mathrm{CO}_{2} \underline{\mathrm{C}}(\mathrm{CH})_{3}\right), 116.48(\mathrm{C}$, arom), $118.16(\mathrm{CH}$, arom), $121.75(\mathrm{CH}$, arom), $125.38(\mathrm{CH}$, arom), $128.52(\mathrm{CH}$, arom), 132.93 (C, arom), 135.25 (C, arom), 149.52 (C, $\left.\underline{\mathrm{CO}}_{2} \mathrm{C}\left(\mathrm{CH}_{3}\right)_{3}\right), 151.75\left(\mathrm{C}, \underline{\mathrm{CO}}_{2} \mathrm{CH}_{3}\right), 170.86\left(\mathrm{C}, \underline{\mathrm{CO}}_{2} \mathrm{CH}_{3}\right)$. Analysis for $\mathrm{C}_{33} \mathrm{H}_{49} \mathrm{BN}_{2} \mathrm{O}_{10}$ : calcd. $[\mathrm{M}+\mathrm{Na}]^{+}(\mathrm{m} / \mathrm{z})$ $=667.3378(100.0 \%), 668.3412(26.0 \%), 666.3414(24.8 \%)$; ESI-Q-TOF HR-ESI-MS: found $[\mathrm{M}+\mathrm{Na}]^{+}=$ $667.3386(100 \%), 668.3413(33.0 \%), 666.3411(20.6 \%) . \delta=1.2 \mathrm{ppm}$.

3-(2-azidodeamino) tryptophan methyl ester 17

1) Methyl ester introduction

A round-bottomed two-neck flask $(500 \mathrm{~mL})$ was charged with tryptophan $(27 \mathrm{~g}, 136 \mathrm{mmol})$ and $\mathrm{CH}_{3} \mathrm{OH}$ $(250 \mathrm{~mL})$ under $\mathrm{N}_{2}$. After cooling with an ice bath, $\mathrm{SOCl}_{2}(29 \mathrm{~mL}, 408 \mathrm{mmol}, 3 \mathrm{eq})$ was added. The mixture was then refluxed. TLC $\left(\mathrm{CH}_{3} \mathrm{OH} / \mathrm{CHCl}_{3} 4: 6\right)$ indicated the consumption of the starting material $\left(\mathrm{R}_{f}=0.38\right)$. After $2 \mathrm{~h}$, the mixture was concentrated by distillation under reduced pressure to give a pink solid.

2) Generation of $\mathrm{TfN}_{3}{ }^{11}$ 
A mixture of $\mathrm{NaN}_{3}(79.7 \mathrm{~g}, 1.2 \mathrm{~mol})$ and a asolution of $\mathrm{H}_{2} \mathrm{O} / \mathrm{CH}_{2} \mathrm{Cl}_{2}$ 1:1 (300 mL) was stirred. After cooling the mixture using ice bath, a mixture of $\mathrm{Tf}_{2} \mathrm{O}(50 \mathrm{~g}, 177 \mathrm{mmol})$ in $\mathrm{CH}_{2} \mathrm{Cl}_{2}(20 \mathrm{~mL})$ was added. The mixture was vigorously stirred for $2 \mathrm{~h}$. It was then partitioned and the organic layer was collected. Aqueous layer was back extracted and the organic layer combined was washed with $\mathrm{NaHCO}_{3}(50 \mathrm{~mL})$ twice. The mixture was cooled at ice bath standby.

3) Azido transfer

The above mixture was then redisolved in $\mathrm{CH}_{3} \mathrm{OH}(50 \mathrm{~mL})$. Followed by addition of $\mathrm{K}_{2} \mathrm{CO}_{3}(37 \mathrm{~g}, 272$ mmol, 2eq), $\mathrm{CuSO}_{4}$ (433 mg, $\left.2.7 \mathrm{mmol}, 0.02 \mathrm{eq}\right), \mathrm{H}_{2} \mathrm{O}(200 \mathrm{~mL})$ and the above solution of $\mathrm{TfN}_{3}$ in $\mathrm{CH}_{2} \mathrm{Cl}_{2}$. TLC $\left(\mathrm{CH}_{3} \mathrm{OH} / \mathrm{CHCl}_{3} 4: 6\right)$ indicated the formation of product $17\left(\mathrm{R}_{f}=0.88\right)$. After $3 \mathrm{~h}$, the mixture was cocentrated under reduced pressure at $40{ }^{\circ} \mathrm{C}$. The residue was partitioned between EtOAc $(150 \mathrm{~mL})$ and $\mathrm{H}_{2} \mathrm{O}$ $(70 \mathrm{~mL} \times 2)$. The organic layer was collected and dired with $\mathrm{MgSO}_{4}$. Followed by filtration through celite, the filtrate was concentrated under reduced pressur. The residue was purified by flash chromatography using grdient mode (EtOAc/n-hexane $1: 9 \rightarrow 1.5: 8.5)$ to give a yellow viscous gum in 69\% yield $(23 \mathrm{~g})$. Spectroscopic data was consistent with the literature data. ${ }^{12}$

${ }^{1} \mathrm{H}-\mathrm{NMR}\left(500 \mathrm{MHz}, \mathrm{CDCl}_{3}\right) \delta 3.21\left(\mathrm{dd}, J_{1 a^{\prime}, 1 b^{\prime}}=15.0, J_{1 a^{\prime} 2^{\prime}}=8.5 \mathrm{~Hz}, 1 \mathrm{H}, \mathrm{H}-1 \mathrm{a}^{\prime}\right), 3.37\left(\mathrm{dd}, J_{1 b^{\prime}, 1 a^{\prime}}=15.0, J_{1 b^{\prime}, 2^{\prime}}\right.$ $\left.=5.5 \mathrm{~Hz}, 1 \mathrm{H}, \mathrm{H}-1 \mathrm{~b}^{\prime}\right), 3.76\left(\mathrm{~s}, 3 \mathrm{H}, \mathrm{CO}_{2} \mathrm{C}_{3}\right), 4.19\left(\mathrm{dd}, J_{2^{\prime}, 1 a^{\prime}}=8.5 \mathrm{~Hz}, J_{2^{\prime}, 1 b^{\prime}}=5.5 \mathrm{~Hz}, 1 \mathrm{H}, \mathrm{H}-2^{\prime}\right), 7.12(\mathrm{~d}, J=$ $2.5 \mathrm{~Hz}, 1 \mathrm{H}$, arom, H-2), 7.15 (t, $J=7.0 \mathrm{~Hz}, 1 \mathrm{H}$, arom, H-5), $7.22(\mathrm{t}, J=7.0 \mathrm{~Hz}, 1 \mathrm{H}$, arom, H-6), $7.37(\mathrm{~d}, J=$ $8.0 \mathrm{~Hz}, 1 \mathrm{H}$, arom, H-7), 7.61 (d, $J=8.0 \mathrm{~Hz}, 1 \mathrm{H}$, arom, H-4), 8.10 (bs, $1 \mathrm{H}, \mathrm{NH}$, arom, H-1). ${ }^{13} \mathrm{C}-\mathrm{NMR}(125$ $\left.\mathrm{MHz}, \mathrm{CDCl}_{3}\right) \delta 27.66\left(\mathrm{CH}_{2}, \mathrm{C}-1^{\prime}\right), 52.60\left(\mathrm{CH}_{3}, \mathrm{CO}_{2} \mathrm{CH}_{3}\right), 62.50(\mathrm{CH}, \mathrm{C}-2$ '), $110.12(\mathrm{C}$, arom, C-3), 111.29 $(\mathrm{CH}$, arom, C-7), $118.40(\mathrm{CH}$, arom, C-5), $119.72(\mathrm{CH}$, arom, C-2), $122.30(\mathrm{CH}$, arom, C-6), $123.17(\mathrm{CH}$, arom, C-4), 127.05 (C, arom, C-9), 136.11 (C, arom, C-8), 170.77 (C, carbonyl). Analysis for $\mathrm{C}_{12} \mathrm{H}_{12} \mathrm{~N}_{4} \mathrm{O}_{2}$ : 
calcd. $(\mathrm{m} / \mathrm{z})[\mathrm{M}+\mathrm{Na}]^{+}=267.0858(100.0 \%), 268.0892$ (13.0\%), 268.0828 (1.5\%); ESI-Q-TOF HR-ESI-MS: found $[\mathrm{M}+\mathrm{Na}]^{+}=267.0858(100 \%), 268.0884(11.7 \%) . \delta=0 \mathrm{ppm}$.

3-(2-azidodeamino)-2,7-diboronopinacol tryptophan methyl ester (AZ-2,7-DBT) 6, 3-(2azidodeamino)-2-boronopinacol tryptophan methyl ester (2-BAT) 18

The borylation reaction was similar to the method as described above except the use of complex of $[\mathrm{Ir}(\mathrm{OMe}) \operatorname{cod}]_{2}(16 \mathrm{mg}, 3 \mathrm{~mol} \%)$. All the reagents have been dried if they can be dissolved in toluene. THF was bubbled by $\mathrm{N}_{2}$ for $15 \mathrm{~min}$. To the flask containing [ $\left.\operatorname{Ir}(\mathrm{OMe}) \operatorname{cod}\right]_{2}(16 \mathrm{mg}, 3 \% \mathrm{~mol})$ was added a mixture of $\mathrm{Me}_{4}$ Phen $\mathrm{Me}_{4}$ Phen $(12 \mathrm{mg}, 6 \% \mathrm{~mol})$ in THF $(1 \mathrm{~mL})$ and a mixture of $\mathrm{B}_{2} \mathrm{pin}_{2}(200 \mathrm{mg}, 0.81 \mathrm{mmol}, 2 \mathrm{eq})$ in THF $(1 \mathrm{~mL})$, sequentially. The mixture was refluxed at $80{ }^{\circ} \mathrm{C}$. The mixture turned dark red and the stirring was allowed for $20 \mathrm{~min}$. A solution of the starting material 17 (100 mg, $0.41 \mathrm{mmol}, 1 \mathrm{eq})$ in THF (1 mL) was added. TLC (acetone/n-hexane 2:8) indicated the consumption of starting material $17\left(\mathrm{R}_{f}=0.30\right)$ and formation of the diborono product AZ-2,7-DBT $6\left(\mathrm{R}_{f}=0.42\right)$ and monoborono product BAT $18\left(\mathrm{R}_{f}=0.62\right)$.

After $1 \mathrm{~h}, \mathrm{CH}_{3} \mathrm{OH}$ was added. The mixture was concentrated under reduced pressure. The residue was purified using flash chromatography with a gradient mode (acetone/ $n$-hexane 1:39 $\rightarrow 1: 29$ ) to give both the aroma odor pale yellow viscous gum in 11\% yield for AZ-2,7-DBT 6 (45 mg) and BAT 18 (34 mg), respectively.

\section{AZ-2,7-DBT 6}

${ }^{1} \mathrm{H}-\mathrm{NMR}\left(500 \mathrm{MHz}, \mathrm{CDCl}_{3}\right) \delta 1.38\left(\mathrm{~s}, 12 \mathrm{H}, \mathrm{CH}_{3}\right), 1.40\left(\mathrm{~s}, 12 \mathrm{H}, \mathrm{CH}_{3}\right), 3.52\left(\mathrm{dd}, J_{1 a^{\prime}, 1 b^{\prime}}=14.0, J_{1 a^{\prime} 2^{\prime}}=8.0 \mathrm{~Hz}\right.$, $\left.1 \mathrm{H}, \mathrm{H}-1 \mathrm{a}^{\prime}\right), 3.56\left(\mathrm{dd}, J_{1 b^{\prime}, 1 a^{\prime}}=14.0, J_{1 b^{\prime}, 2^{\prime}}=7.0 \mathrm{~Hz}, 1 \mathrm{H}, \mathrm{H}-1 \mathrm{~b}^{\prime}\right), 3.64\left(\mathrm{~s}, 3 \mathrm{H}, \mathrm{CO}_{2} \mathrm{CH}_{3}\right), 4.19\left(\mathrm{dd}, J_{2^{\prime}, 1 a^{\prime}}=8.0\right.$, $\left.J_{2^{\prime}, l b^{\prime}}=7.0 \mathrm{~Hz}, 1 \mathrm{H}, \mathrm{H}-2^{\prime}\right), 7.11(\mathrm{t}, J=7.0 \mathrm{~Hz}$, arom, H-5), 7.69 (d, $J=7.0 \mathrm{~Hz}$, arom, H-4), 7.75 (d, $J=8.0 \mathrm{~Hz}$, arom, H-6), 9.28 (bs, arom, NH). ${ }^{13} \mathrm{C}-\mathrm{NMR}\left(125 \mathrm{MHz}, \mathrm{CDCl}_{3}\right) \delta 25.60\left(\mathrm{CH}_{3}, \mathrm{Bpin}\right), 28.76\left(\mathrm{CH}_{2}, \mathrm{C}-1^{\prime}\right), 53.09$ $\left(\mathrm{CH}_{2}, \mathrm{CO}_{2} \underline{\mathrm{CH}} 3\right), 64.38\left(\mathrm{CH}, \mathrm{C}-2^{\prime}\right), 84.53\left(\mathrm{CH}_{3} \underline{\mathrm{CCH}}_{3}\right), 84.72\left(\mathrm{CH}_{3} \underline{\mathrm{CCH}_{3}}\right), 119.88(\mathrm{CH}$, arom, $\mathrm{C}-5), 122.89(\mathrm{C}$, 
arom, C-3), 123.65 (CH, arom, C-4), 127.66 (C, arom, C-9), 128.92 (C, arom, C-2) 129.72 (C, arom, C-7), $132.19\left(\mathrm{CH}\right.$, arom, C-6), $143.51\left(\mathrm{C}\right.$, arom, C-8), $171.83\left(\underline{\mathrm{CO}}_{2} \mathrm{CH}_{3}\right)$. Analysis for $\mathrm{C}_{24} \mathrm{H}_{34} \mathrm{~B}_{2} \mathrm{~N}_{4} \mathrm{O}_{6}$ : calcd. $(\mathrm{m} / \mathrm{z})$ $[\mathrm{M}+\mathrm{Na}]^{+}=519.2562(100.0 \%), 518.2598(49.7 \%), 520.2596$ (26.0\%), ESI-Q-TOF HR-ESI-MS: found $[\mathrm{M}+\mathrm{Na}]^{+}=519.2560(100 \%), 518.2590(38.3 \%), 520.2586(21.7 \%) . \delta=-0.4 \mathrm{ppm}$.

\section{2-BAT 18}

${ }^{1} \mathrm{H}-\mathrm{NMR}\left(500 \mathrm{MHz}, \mathrm{C}_{6} \mathrm{D}_{6}\right) \delta 1.10\left(\mathrm{~s}, 6 \mathrm{H}, \mathrm{CH}_{3}\right), 1.11\left(\mathrm{~s}, 6 \mathrm{H}, \mathrm{CH}_{3}\right), 3.13\left(\mathrm{~s}, 3 \mathrm{H}, \mathrm{CO}_{2} \underline{\mathrm{CH}_{3}}\right), 3.73\left(\mathrm{~d}, J_{1^{\prime}, 2^{\prime}}=7.0\right.$ Hz, 2H, H-1'), 4.25 (t, $\left.J_{2^{\prime}, l^{\prime}}=7.0 \mathrm{~Hz}, 1 \mathrm{H}, \mathrm{H}-2^{\prime}\right), 6.94$ (d, J=8.0 Hz, 1H, arom, H-7), 7.08 (t, J=7.0 Hz, 1H, arom, H-5), 7.18 (t, $J=6.0 \mathrm{~Hz}, 1 \mathrm{H}$, arom, H-6), 7.69 (d, $J=8.0 \mathrm{~Hz}, 1 \mathrm{H}$, arom, H-4), 7.92 (bs, 1H, NH, arom, H-1), ${ }^{13} \mathrm{C}-\mathrm{NMR}\left(125 \mathrm{MHz}, \mathrm{C}_{6} \mathrm{D}_{6}\right) \delta 24.73\left(\mathrm{CH}_{3}, \mathrm{Bpin}\right), 28.41\left(\mathrm{CH}_{2}, \mathrm{C}-1\right.$ ') $, 51.74\left(\mathrm{C}, \mathrm{CO}_{2} \underline{\mathrm{CH}}_{3}\right), 63.57$ $\left(\mathrm{CH}, \mathrm{C}-2^{\prime}\right), 84.02\left(\mathrm{C}, \mathrm{CH}_{3} \underline{\mathrm{CCH}}_{3}\right), 111.83(\mathrm{CH}, \operatorname{arom}), 120.00(\mathrm{CH}$, arom $), 123.11(\mathrm{C}$, arom), $124.17(\mathrm{CH}$, arom), $138.55\left(\mathrm{C}\right.$, arom), $170.87\left(\mathrm{C}, \underline{\mathrm{CO}}_{2} \mathrm{CH}_{3}\right.$, carbonyl). Analysis for $\mathrm{C}_{18} \mathrm{H}_{23} \mathrm{BN}_{4} \mathrm{O}_{4}$ : calcd. $(\mathrm{m} / \mathrm{z})[\mathrm{M}+\mathrm{Na}]^{+}=$ $393.1710(100.0 \%), 392.1746$ (24.8\%), 394.1744 (19.5\%), ESI-Q-TOF HR-ESI-MS: found [M+Na] $]^{+}=$ $393.1707(100.0 \%), 392.1742(21.6 \%), 394.1737(17.6 \%) . \delta=-0.8 \mathrm{ppm}$.

\subsection{Bioassay}

\subsubsection{Cell survival assay}

U87-MG, LN229 and 3T3- Fibroblast cell lines were purchased from ATCC. The three cell lines were each planted in a 96-well microtiter plate in a concentration of 5000/100 uL. A stock solution of the tryptophan derivatives and the control compound BPA were prepared by disolving in DMSO to $10 \mathrm{mM}$ (1.68 $-2.39 \mathrm{~mL})$. A volume of $0.1 \mathrm{~mL}$ each stock solution was mixed with $0.9 \mathrm{~mL}$ of saline. An aliquote $(0.1 \mathrm{~mL})$ was further diluted using $0.9 \mathrm{~mL}$ of medium to generate a mixture in concentration of $100 \mathrm{uM}$. Repeating this procedure for a series of dilution, samples with various concentrations ranging from $1 \mathrm{nM}$ to $100 \mathrm{uM}$ were obtained. The above mentioned cell-planted medium (100 uM) was removed and replenished by the 
same volume of corresponding dilution of the tryptophan derivatives and BPA. After incubation for $24 \mathrm{~h}$, the WST-1 agent (10 uL) was added. After 2-h standing, the plates were submitted to Eliser reader for detection at $\lambda_{\max }=450 \mathrm{~nm}$. The survival ratio was calculated as optical density of the group treated with compounds devided by the group treated with $0.1 \%$ DMSO and multiplied by $\%$. Data were obtained in triplicate.

\subsubsection{Cell accumulation assay}

The three cell lines were seeded in a petri dish (ID: $10 \mathrm{~cm}$ ) containing a medium of $10 \mathrm{~mL}$ in a concentration of $\sim 5 \times 10^{5}$ cells/well. After adhesion to the inner wall for $24 \mathrm{~h}$, the medium was replaced by a new medium containing tryptophan derivatives and BPA $(50 \mu \mathrm{M}, 10 \mathrm{~mL})$. The supernatant at each time point of incubation for $0.05,1,2$, and $4 \mathrm{~h}$ was removed and the cells were washed with a PBS solution (5 $\mathrm{mL})$ two times. The residual cell mixture was cleaved using a mixture of trypsin and EDTA ( $2 \mathrm{~mL})$ for 5 min. The mixture of cell pellets was washed with medium $(5 \mathrm{~mL})$ for three times followed by transferring to a centrifuge tube for centrifugation at $1200 \mathrm{rpm}$ for $5 \mathrm{~min}$. The supernatants were removed followed by replenishment of $1 \mathrm{~mL}$ of medium for dissolution. An aliquot of $20 \mathrm{uL}$ was mixed with trypan blue $(20 \mathrm{uL})$ and $10 \mathrm{uL}$ of which was drawn for cellular counting. The centrifuge tube was centrifuged again. The supernatant was removed and the precipitate was treated with $70 \% \mathrm{Wt}$. $\mathrm{HNO}_{3(\mathrm{aq})}(75 \mathrm{uL})$ for digestion for 48h. Additional distilled $\mathrm{H}_{2} \mathrm{O}(1.42 \mathrm{~mL})$ was added to give a concentration of $3.5 \% \mathrm{HNO}_{3}(\mathrm{aq})$ in $1.5 \mathrm{~mL}$. The samples were analyzed for the concentration of born using inductively coupled plasma mass spectrometry (ICP-MS). All the data were obtained in triplicate. The detection limit of the instrument is $0.399 \mathrm{ppb}$.

The concentration of ${ }^{10} \mathrm{~B}$ atoms was calculated based on the following equation:

ug ${ }^{10} \mathrm{~B} / \mathrm{g}=\frac{\mathrm{ppb}^{11} \mathrm{~B} \times 10^{-9} \times 1.5(\mathrm{~mL}) \times 1(\mathrm{~g} / \mathrm{mL}) \times 0.2\left(\mathrm{~g} \text { of }{ }^{10} \mathrm{~B} / \mathrm{g} \text { of }{ }^{11} \mathrm{~B}\right) \times 10^{6}(\mathrm{ug} / \mathrm{g})}{\mathrm{N} \text { cell } \times 10^{-9}(\mathrm{~g} / \text { cell })}$

\subsubsection{Cell eradication assay after neutron irradiation}

The three cell lines were each planted in a 96-well microtiter plate in a concentration of 5000/100 uL. A stock solution was prepared by dissolving the tryptophan derivatives in DMSO to $15 \mathrm{mM}(0.35-0.65 \mathrm{~mL})$. An aliquot of $0.2 \mathrm{~mL}$ was mixed with $0.4 \mathrm{~mL}$ of saline to generate a solution of $5 \mathrm{mM}$ (33\% DMSO) 
followed by mixing an aliquot of $0.3 \mathrm{~mL}$ with $2.7 \mathrm{~mL}$ of saline. The mixture (500 uM, 3.3\% DMSO) was further mixed with medium in 10-fold dilution (50 uM, 0.3\% DMSO). The dilutions (100 uL) were each used to replace the above medium in the well of microtiter plate. After incubation for $1 \mathrm{~h}$, the medium was discarded and the plates were fixed for neutron irradiation. Two plates were parallel placed and were irradiated simultaneously using a neutron flux of $10^{13} \mathrm{n} / \mathrm{s} \mathrm{cm}^{2}$ with a peak power of $1.2 \mathrm{MW}$ for $3 \mathrm{~min}$. The irradiation experiment was carried out in Tsing-Hua Open-Pool Reactor at National Tsinghua University. The total running time was $25 \mathrm{~min}$. The plates were then replenished with medium (100 uL) and WST-1 agent $(10 \mathrm{uL})$ for incubation of $2 \mathrm{~h}$ without waiting for $24 \mathrm{~h}$. The plates were simultaneously submitted to Eliser reader for detection of the absorbance at $\lambda_{\max }=450 \mathrm{~nm}$. The survival ratio was calculated as optical density of the group treated with compounds devided by that of the group treated with $0.1 \%$ DMSO and multiplied by $\%$. Data were obtained in quadreplicate.

\subsubsection{Western blot assay}

U87-MG, LN229 (glioma cell lines) and 3T3 cell (fibroblast cell line) were cultured with DMEM (Corning) and $10 \%$ fetal bovine serum (FBS) and maintained at $37^{\circ} \mathrm{C}$ in a humidified incubator with $5 \%$ $\mathrm{CO}_{2}$. After $80 \%$ of cell confluency, the tryptophan derivatives and the control compound mentioned above $(50 \mathrm{uM})$ were added and incubated for 24 hours. Cells were lysed (RIPA buffer, Thermo Scientific) and supernatants were recovered by centrifugation $(13000 \mathrm{rpm})$, protein concentration was measured, and equal amounts of total protein were separated by sodium dodecyl sulfate-polyacrylamide gel electrophoresis. The proteins were transferred to PVDF (poly-vinylidene difluoride) membrane (Millipore, Bedford, MA) and then blockade for $1 \mathrm{~h}$ in $5 \%$ bovine serum albumin in TBS-T. Membranes were incubated overnight at $4{ }^{\circ} \mathrm{C}$ with primary antibody LAT-1 (Bioss Antibodies, 55Kda, 1:2000). Membranes were incubated with the corresponding secondary antibody (Anti-rabbit IgG, goat) for $1 \mathrm{~h}$. Specific bands were detected using the enhanced chemiluminescence reagent (ECL, PerkinElmer Life Sciences, Boston, MA) on an 
autoradiographic film.

2. Spectra and chromatogram of compounds

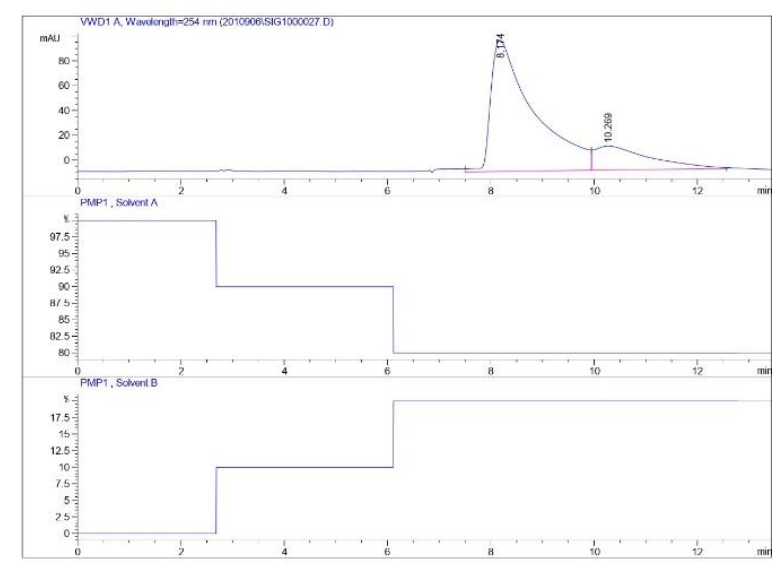<smiles>NC(Cc1c[nH]c2ccc(Br)cc12)C(=O)O</smiles>

Figure 1. HPLC chromatogram of 5-bromo-3-(2R,2S)tryptophan

5-bromo- $d, l$-tryptophan prepared as a concentration of $1 \mathrm{mg} / 1.0 \mathrm{~mL}$ was injected into a reversed-phase HPLC in a portion of $5 \mathrm{uL}$. The flow rate was set at $0.8 \mathrm{~mL} / \mathrm{min}$ for an HPLC column of CHIRALCEL ${ }^{\circledR}$ OD-RH with particle size of $5 \mathrm{um}$ and a column dimension of $4.6 \times 150 \mathrm{~mm}$. Elution was conditioned by using a gradient mode of $\mathrm{H}_{2} \mathrm{O} / \mathrm{EtOH}$ 100:0 $\rightarrow$ 90:10 from 0 to 3 min, $\mathrm{H}_{2} \mathrm{O} / \mathrm{EtOH}$ 90:10 $\rightarrow$ 80:20 from 3 to $6 \mathrm{~min}$, and a constant $\mathrm{H}_{2} \mathrm{O} / \mathrm{EtOH}$ 80:20 from 6 to $20 \mathrm{~min}$. UV detector was set at $\lambda_{\text {abs }}=254$ $\mathrm{nm}$. The two $d, l$ isomers were recorded at $t_{R 1}=8.17 \mathrm{~min}$ and $t_{R 2}=10.27 \mathrm{~min}$, respectively. 
Solvent: cdc13

Tenp. $26.0 \mathrm{C} / 299.1 \mathrm{x}$

INOVA-500 "Varian-NMR

Relax. delay 1.000 sec

Pulse 45.0 degrees

Acq. time $2.046 \mathrm{se}$

Width $7509.6 \mathrm{~Hz}$

128 repetitions

OBSERVE H1, 499

Line broadening $0.5 \mathrm{~Hz}$

FT size 65536

Total time $6 \mathrm{~min}, 37 \mathrm{sec}$
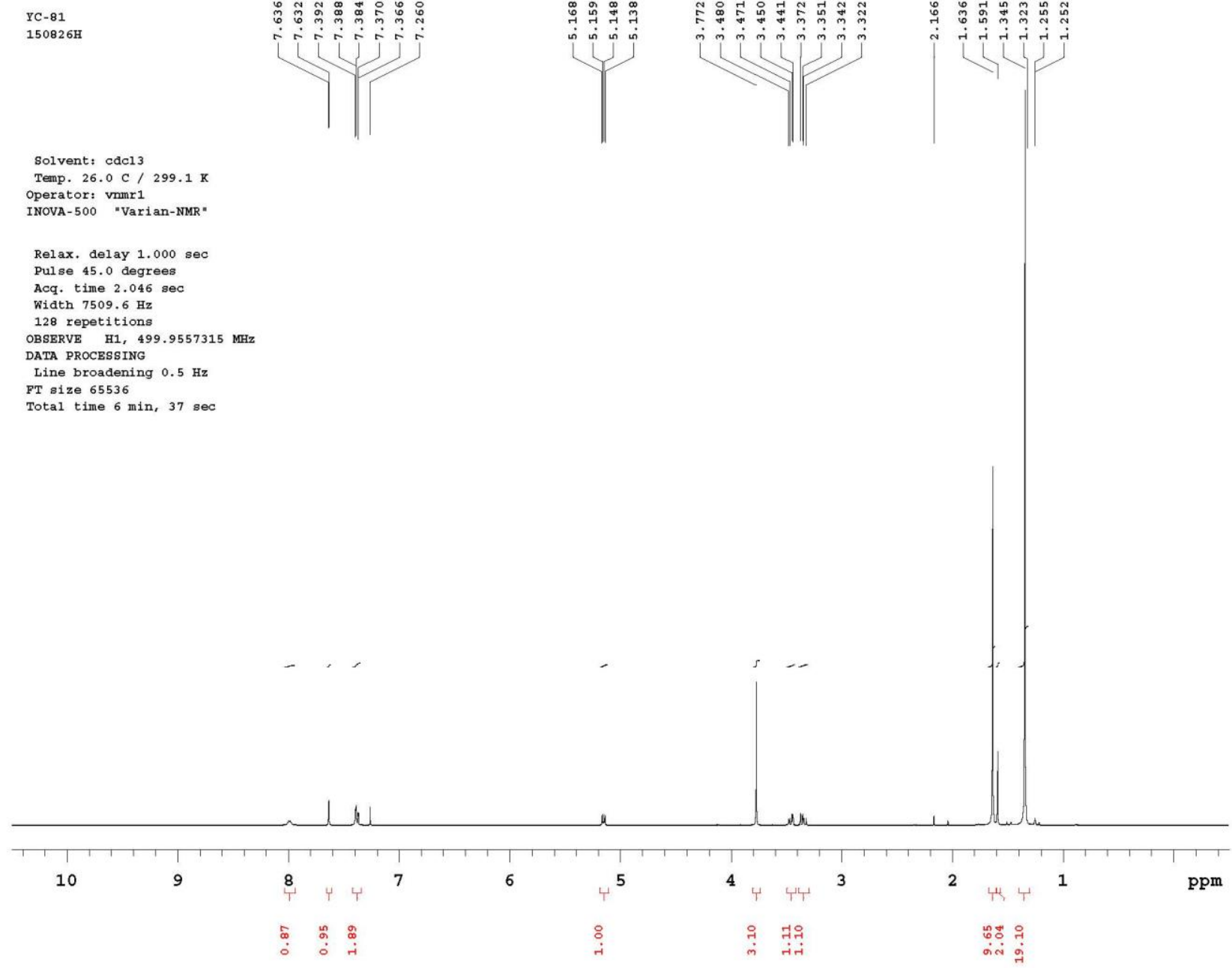<smiles>COC(=O)C(Cc1cn(C(C)(C)C)c2ccc(Br)cc12)NC(=O)OCc1ccccc1</smiles>

${ }^{1} \mathrm{H}-\mathrm{NMR}$ in $\mathrm{CDCl}_{3}$

1,3-(2-N,N)triBoc-5-bromo tryptophan methylester 7 


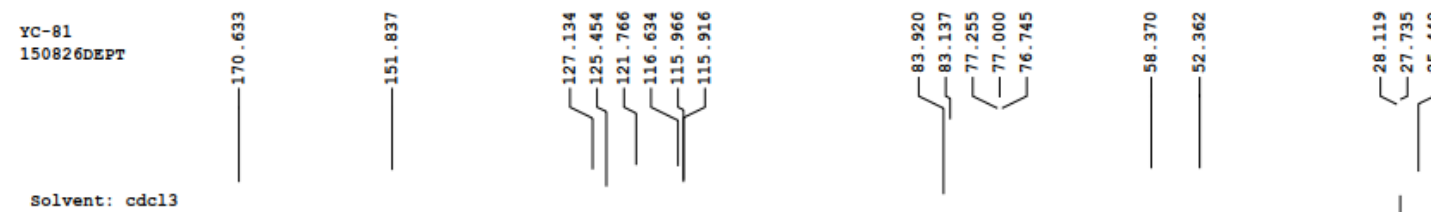

Temp. $26.0 \mathrm{c} / 299.1 \mathrm{~K}$

operator: vnmr1

INOVA-500 "Varian-NMR"

Relax. delay $1.500 \mathrm{sec}$

Pulse 90.0 degrees

Acq. time 1.001 sec

width $28891.3 \mathrm{~Hz}$

BSERVE C13, 125.7139700 MBZ

DEcove c13, 125.7139700 $\mathrm{maz}$

DECOUPLE H1, $499.9582169 \mathrm{MHz}$

on during acquisition

off during delay

WALTZ-16 modula

Line broadening 1.0 Hz

(1)

Total time $1 \mathrm{hr}, 24 \mathrm{~min}, 17 \mathrm{sec}$

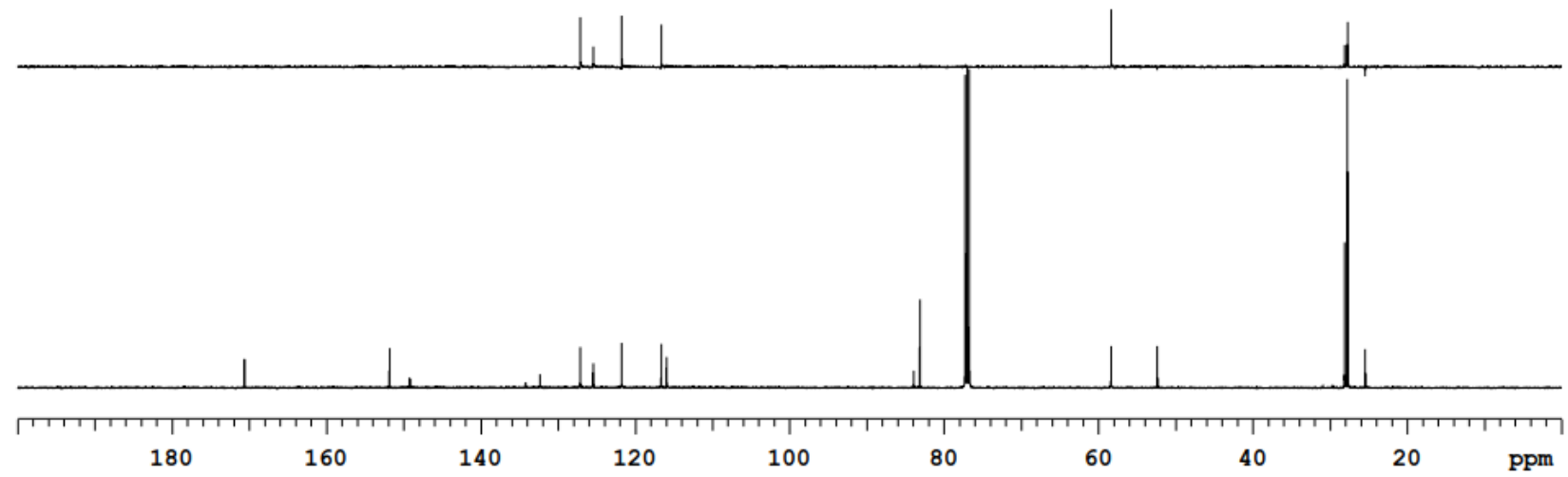

${ }^{13} \mathrm{C}$-DEPT $135 \mathrm{NMR}$ in $\mathrm{CDCl}_{3}$

1,3-(2- $N, N)$ triBoc-5-bromotryptophan methyl ester 7 


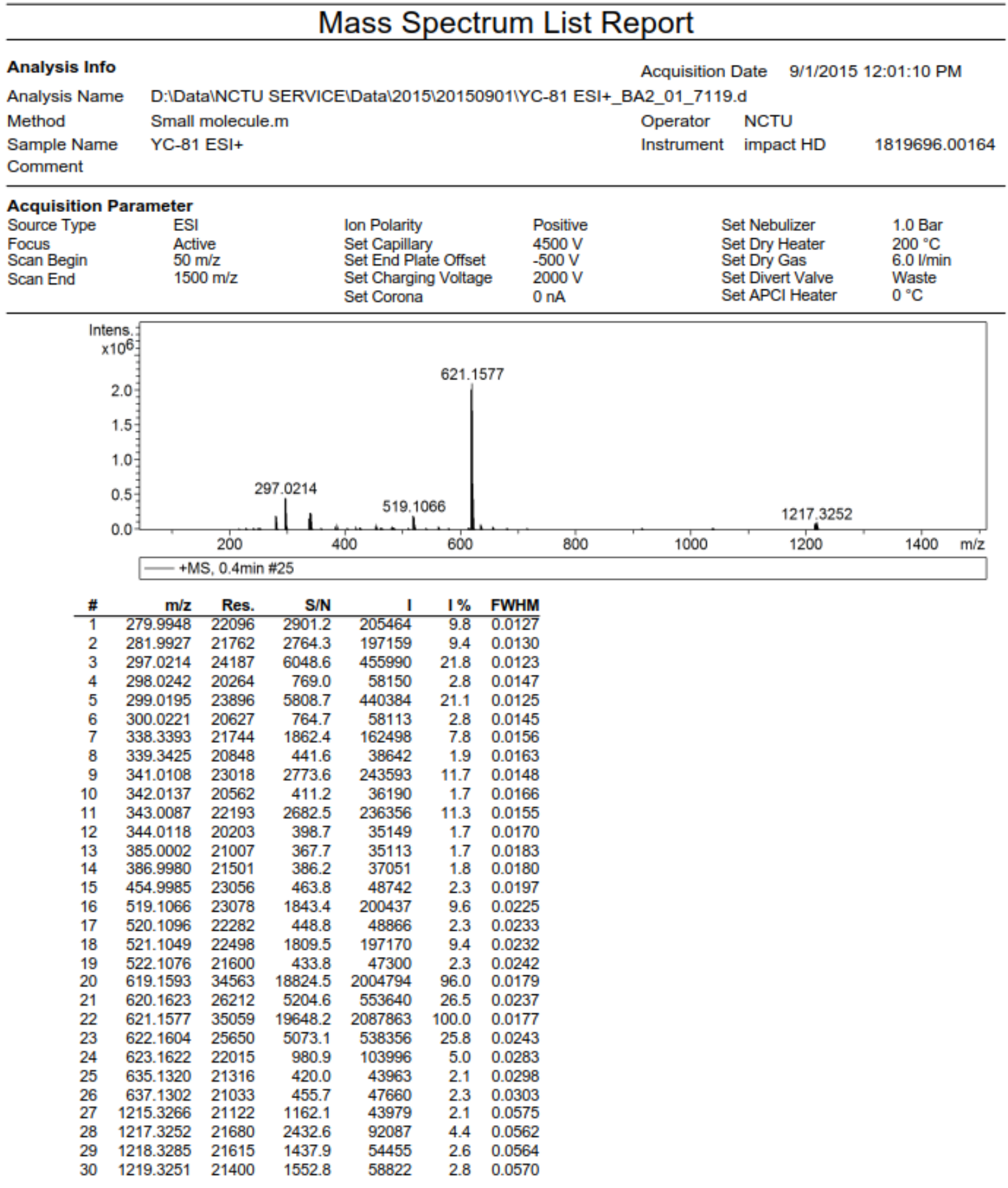

YC-81 ESI+_BA2_01_7119.d

Bruker Compass DataAnalysis $4.1 \quad$ printed: 9/14/2015 10:14:25 AM $\quad$ by: NCTU 1

HR ESI-MS

1,3-(2-N,N)triBoc-5-bromotryptophan methyl ester 7 
Figure 2. Spectra of 1,3-(2-N,N)triBoc-5-bromotryptophan methyl ester 7

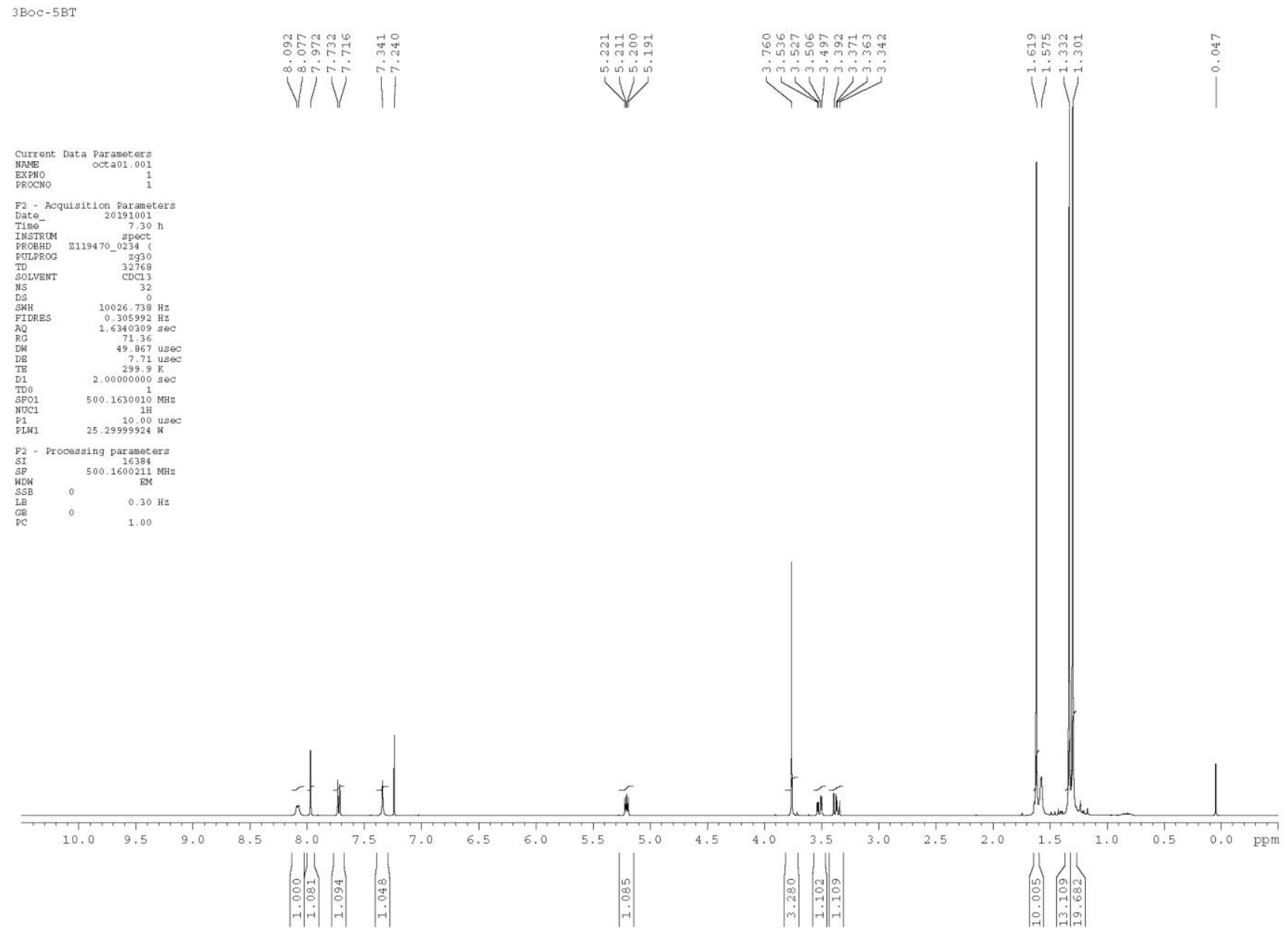

${ }^{1} \mathrm{H}-\mathrm{NMR}$ in $\mathrm{CDCl}_{3}$<smiles></smiles>

TB-5-BT 1 


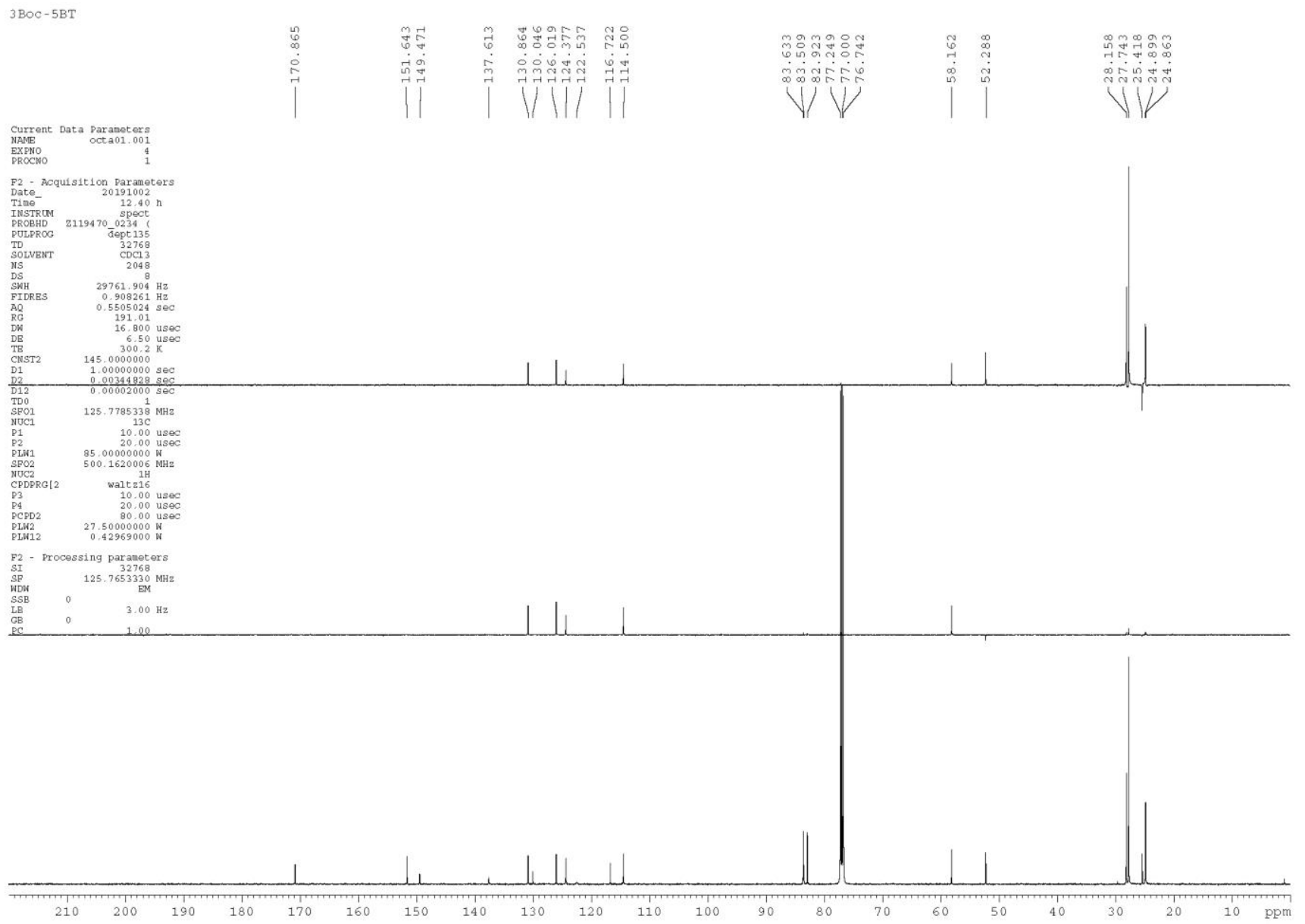

${ }^{13} \mathrm{C}$-DEPT $135 \mathrm{NMR}$ in $\mathrm{CDCl}_{3}$

TB-5-BT 1 
Data:TB-5-BT

Comment:

Description:

Ionization Mode:ESI+

History:Average(MS[1] 0.52..0.55)

Charge number: 1

Tolerance: $250.00[\mathrm{ppm}], 200.00$.. 200... Unsaturation Number:-100.5 .. 200.0 (...

Element: ${ }^{12} \mathrm{C}: 48$.. 48, ${ }^{1} \mathrm{H}: 78$.. 82, ${ }^{14} \mathrm{~N}: 6 . .6,{ }^{23} \mathrm{Na}: 0 . .3,{ }^{16} \mathrm{O}: 32 . .32$

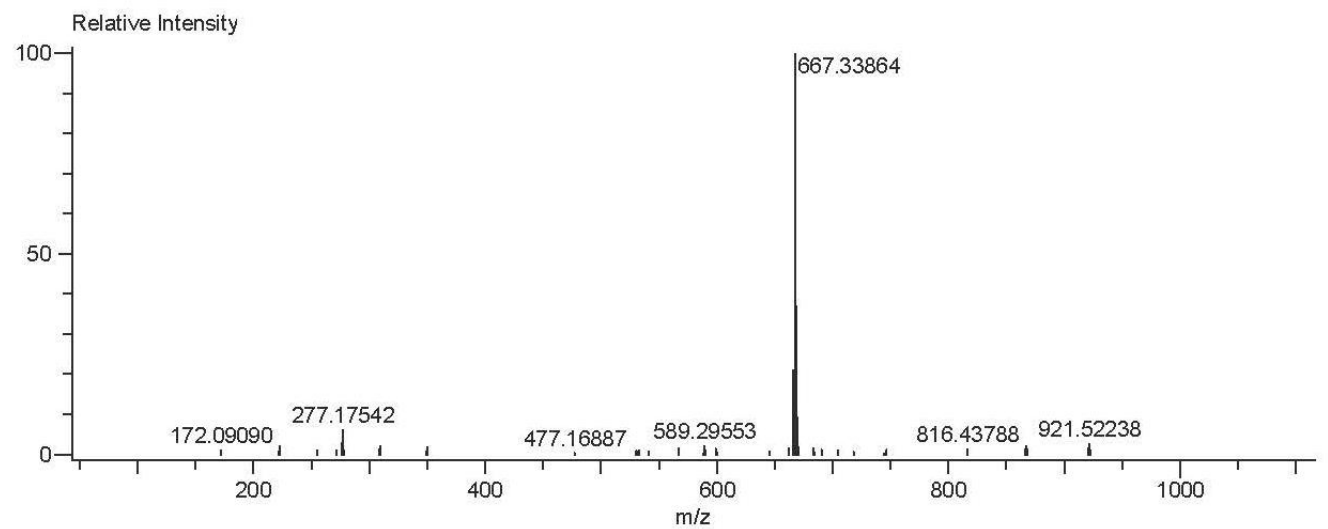

HR ESI-MS

TB-5-BT 1

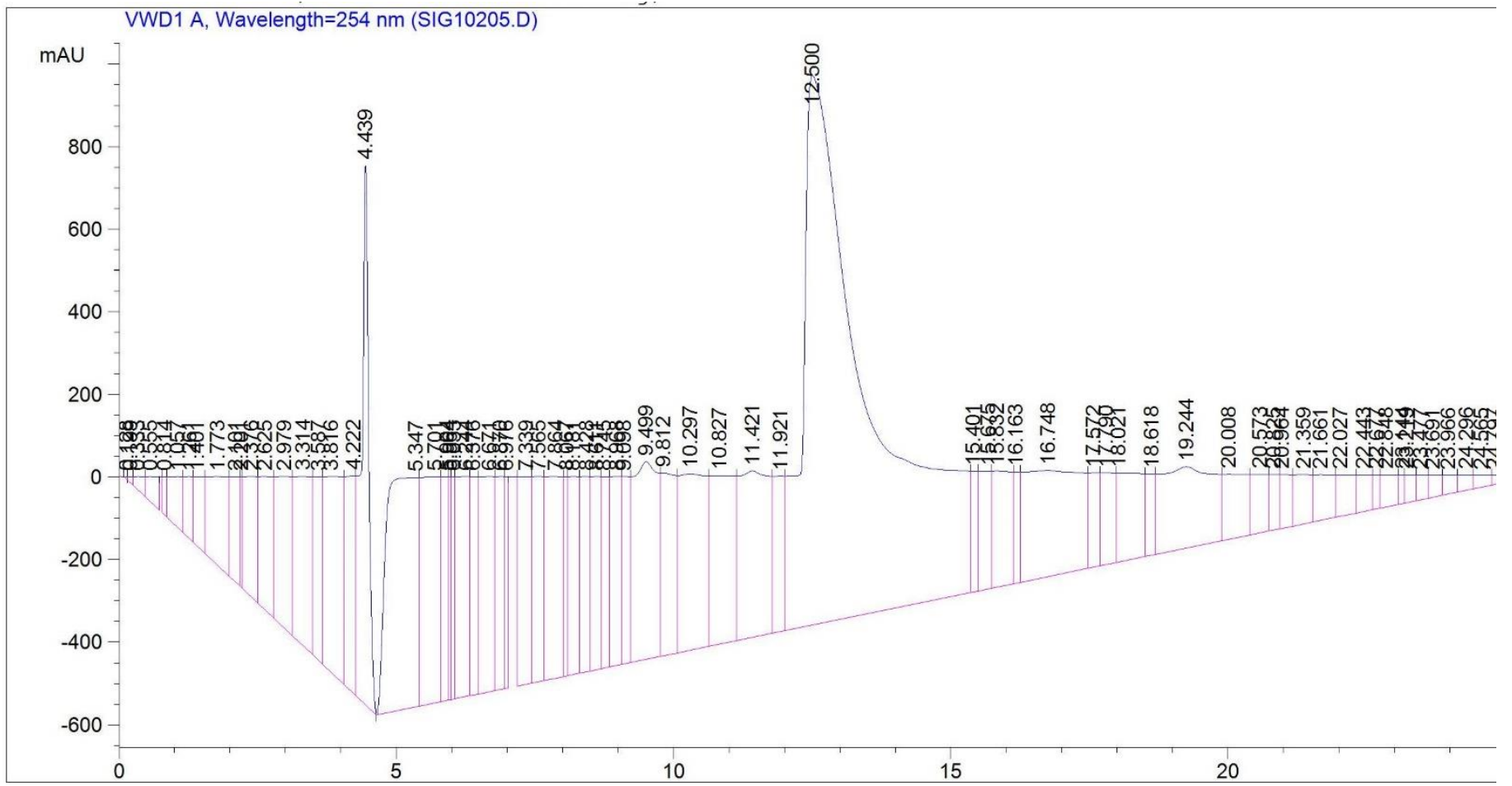

HPLC chromatogram of TB-5-BT 1

EtOAc $/ n$-hexane 1:5, $t_{R}=4.439 \mathrm{~min}$, void solvent; $t_{R}=12.500 \mathrm{~min}$, TB-5-BT 1. Si-90 column HPLC as described in general section.

Figure 3. Spectra and chromatogram of 1,3-(2R,2S)-(2-N,N)triBoc-5-boronopinacoltryptophan methyl ester (TB-5-BT) 1 


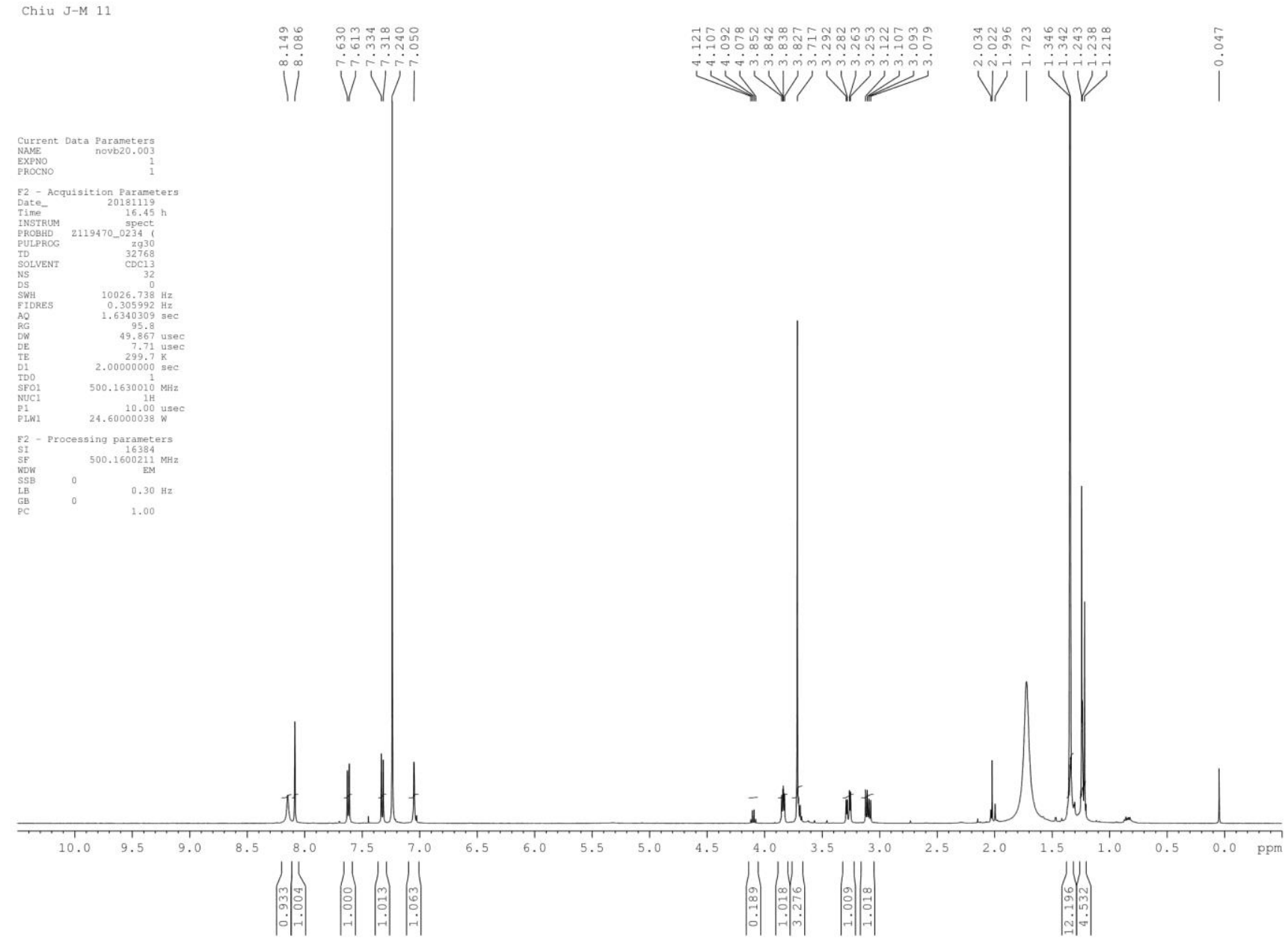

${ }^{1} \mathrm{H}-\mathrm{NMR}$ in $\mathrm{CDCl}_{3}$<smiles>COC(=O)C(N)Cc1c[nH]c2ccc(B3OC(C)(C)C(C)(C)O3)cc12</smiles>

5-BT 4 
Chiu J-M 11
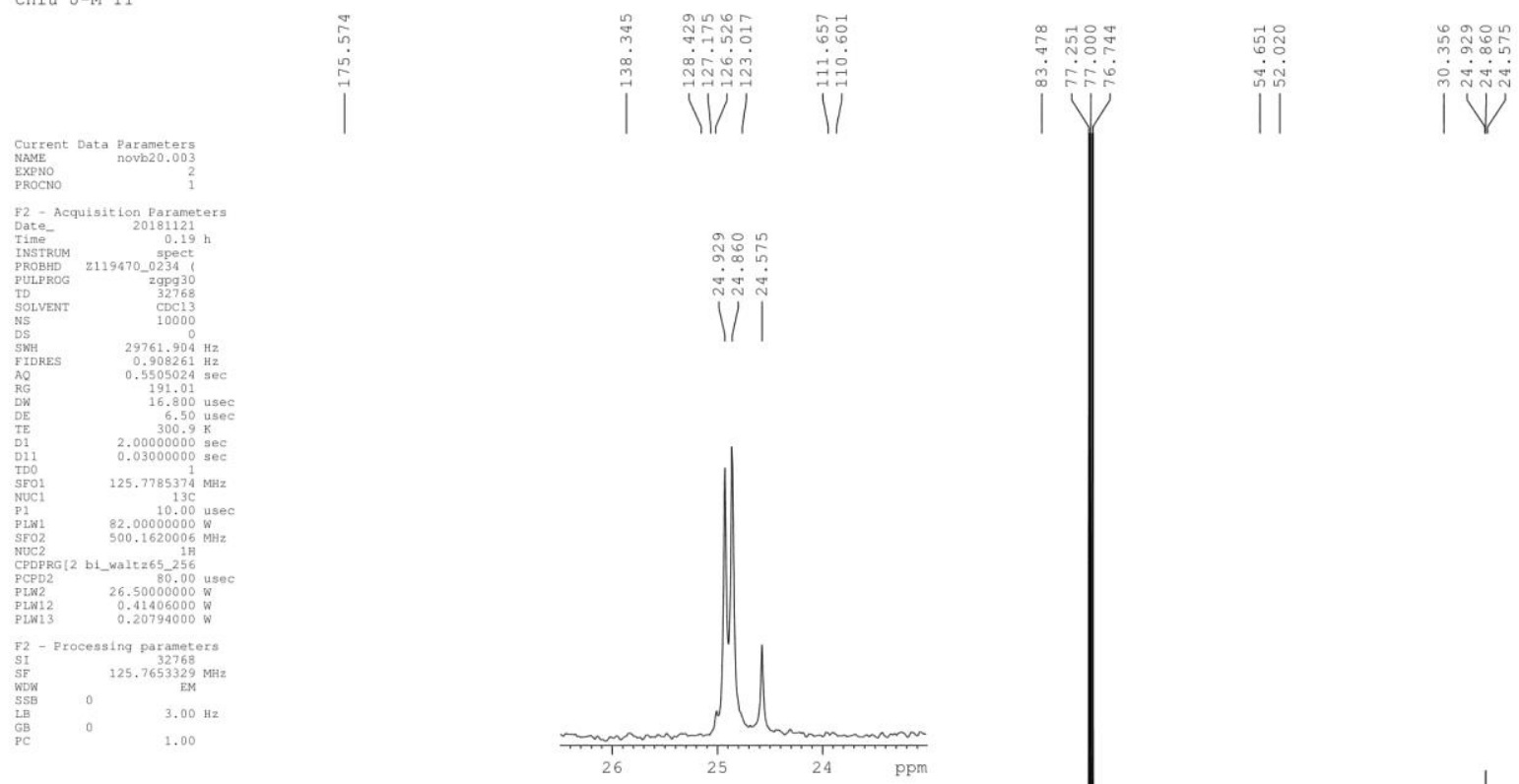

\section{5-BT 4 in $\mathrm{CDCl}_{3}$}




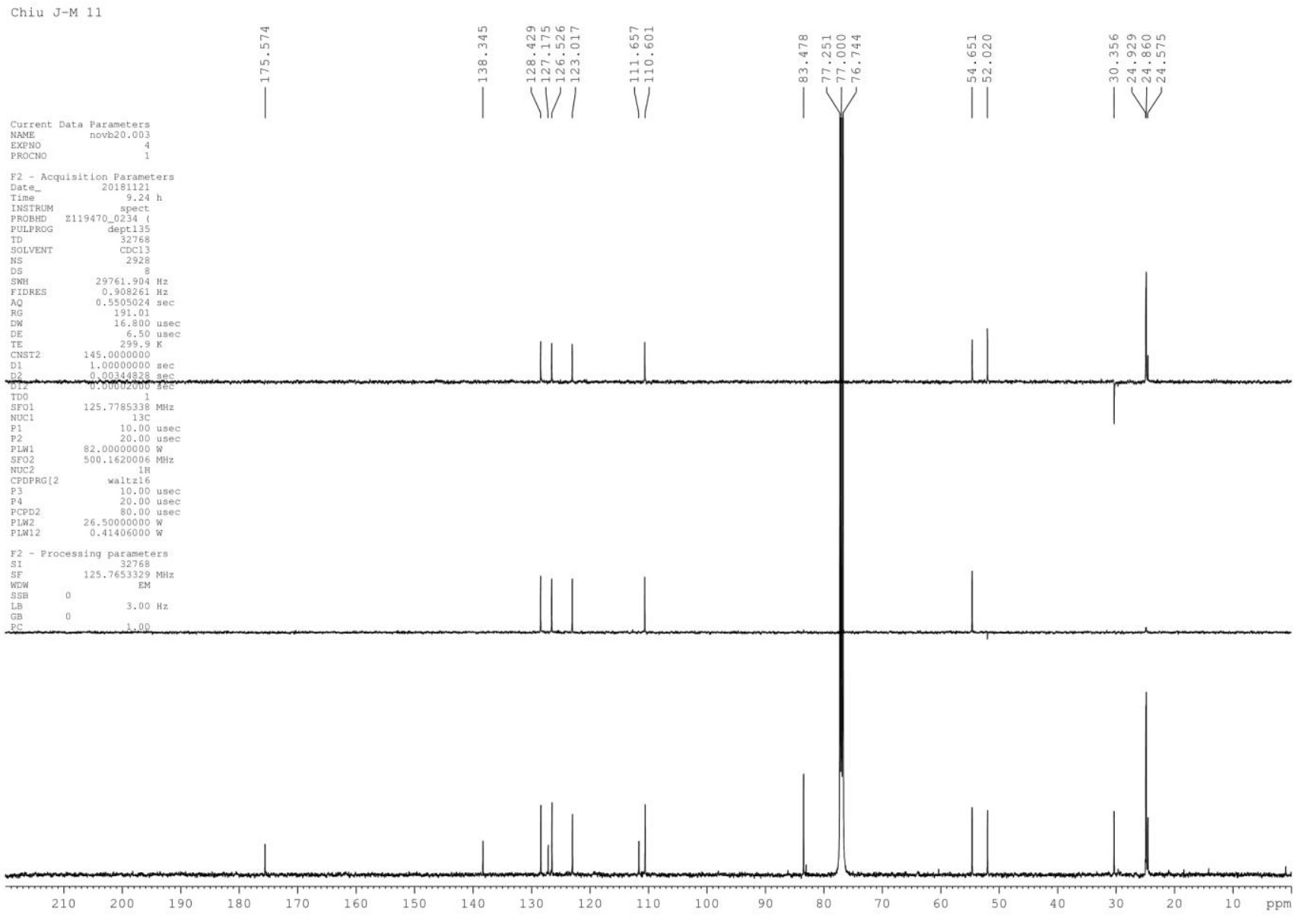

${ }^{13} \mathrm{C}$-DEPT $135 \mathrm{NMR}$ in $\mathrm{CDCl}_{3}$

5-BT 4 


\section{Display Report}
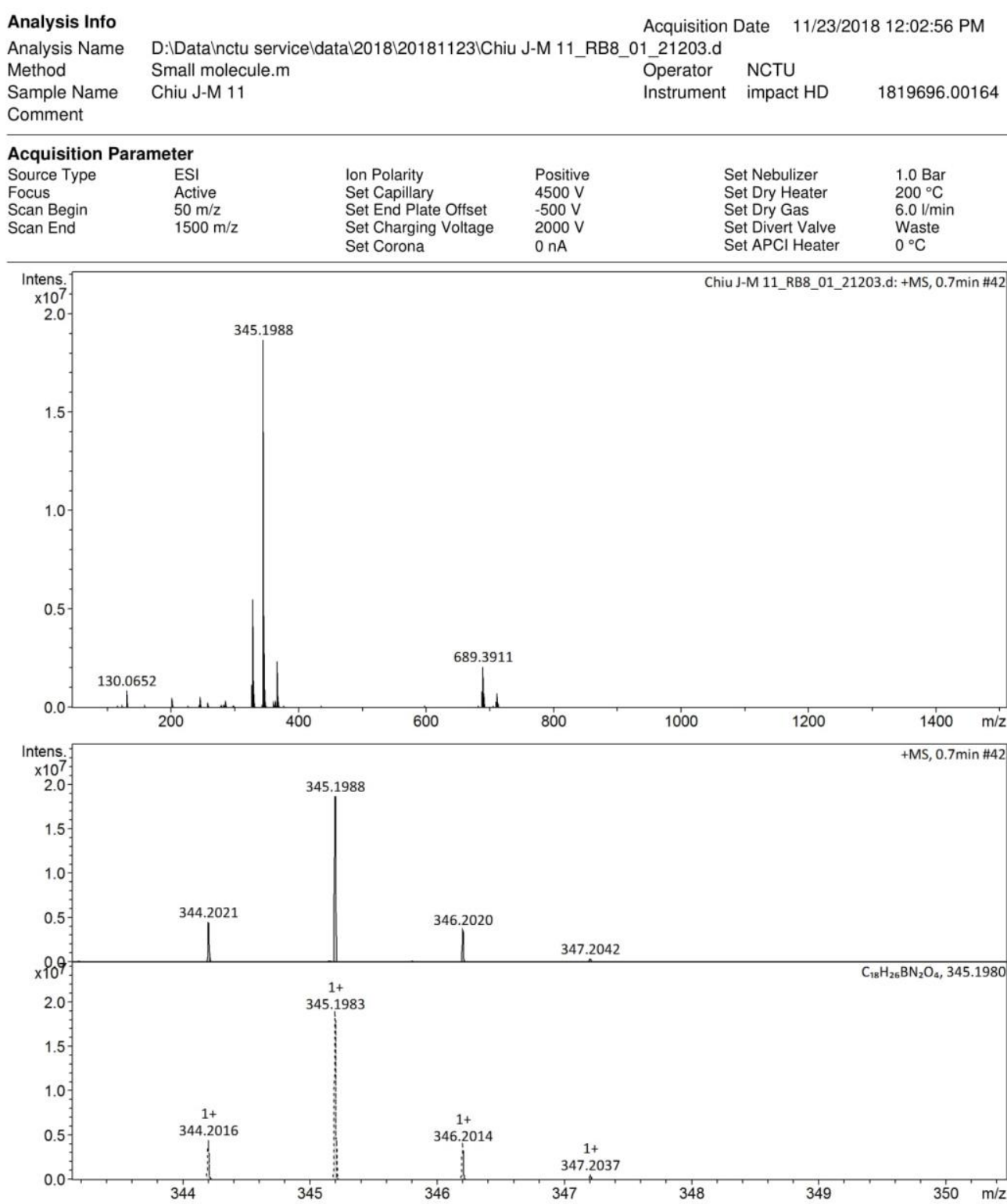

Chiu J-M 11_RB8_01_21203.d 
Figure 4. Spectra of 3-(2R,2S)-5-boronopiacoltryptophan methyl ester (5-BT) 4

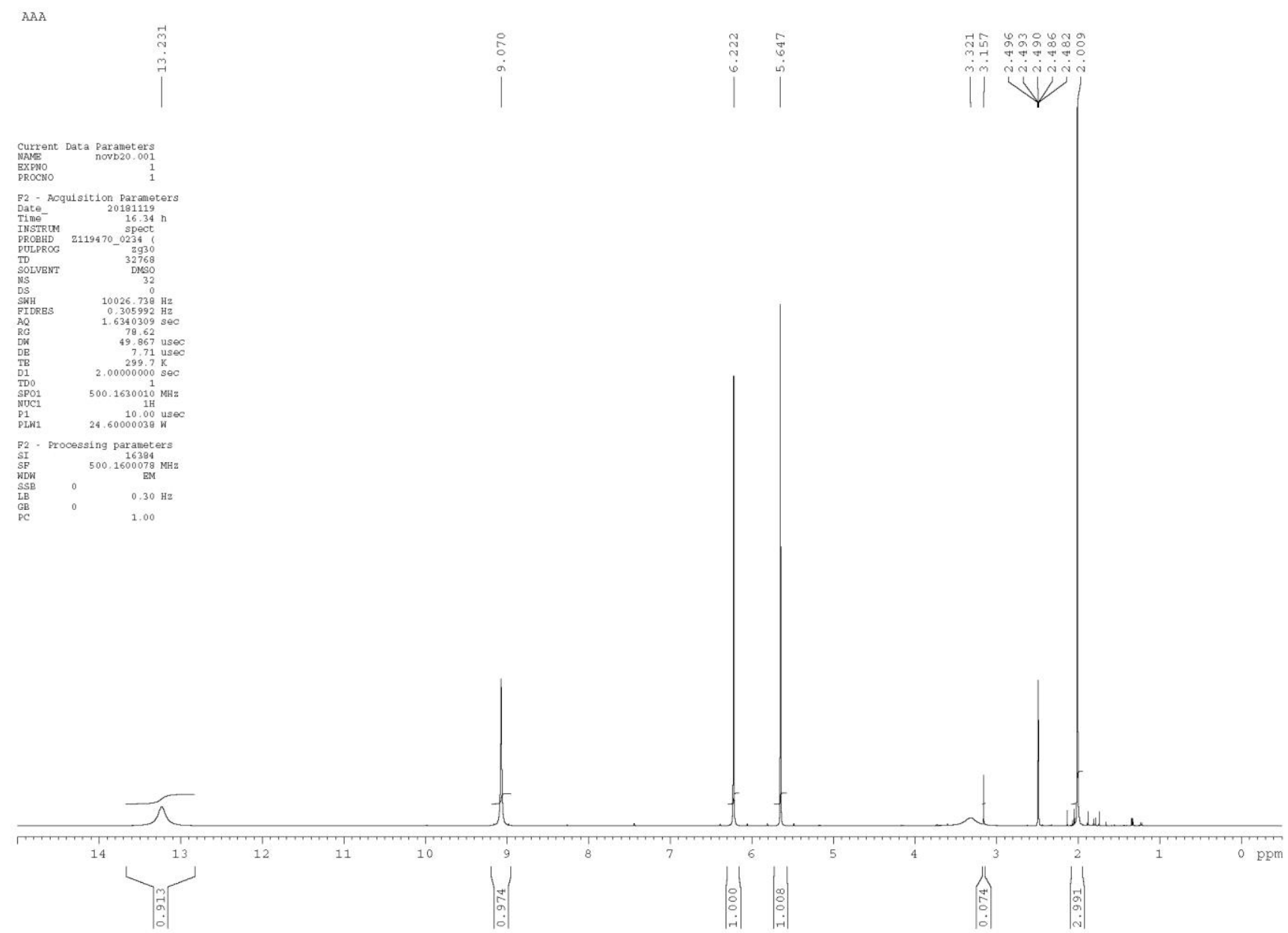

${ }^{1} \mathrm{H}-\mathrm{NMR}$ in DMSO-d 6<smiles>C=C(NC(C)=O)C(=O)O</smiles>

2-(acetylamino)acrylic acid 8 


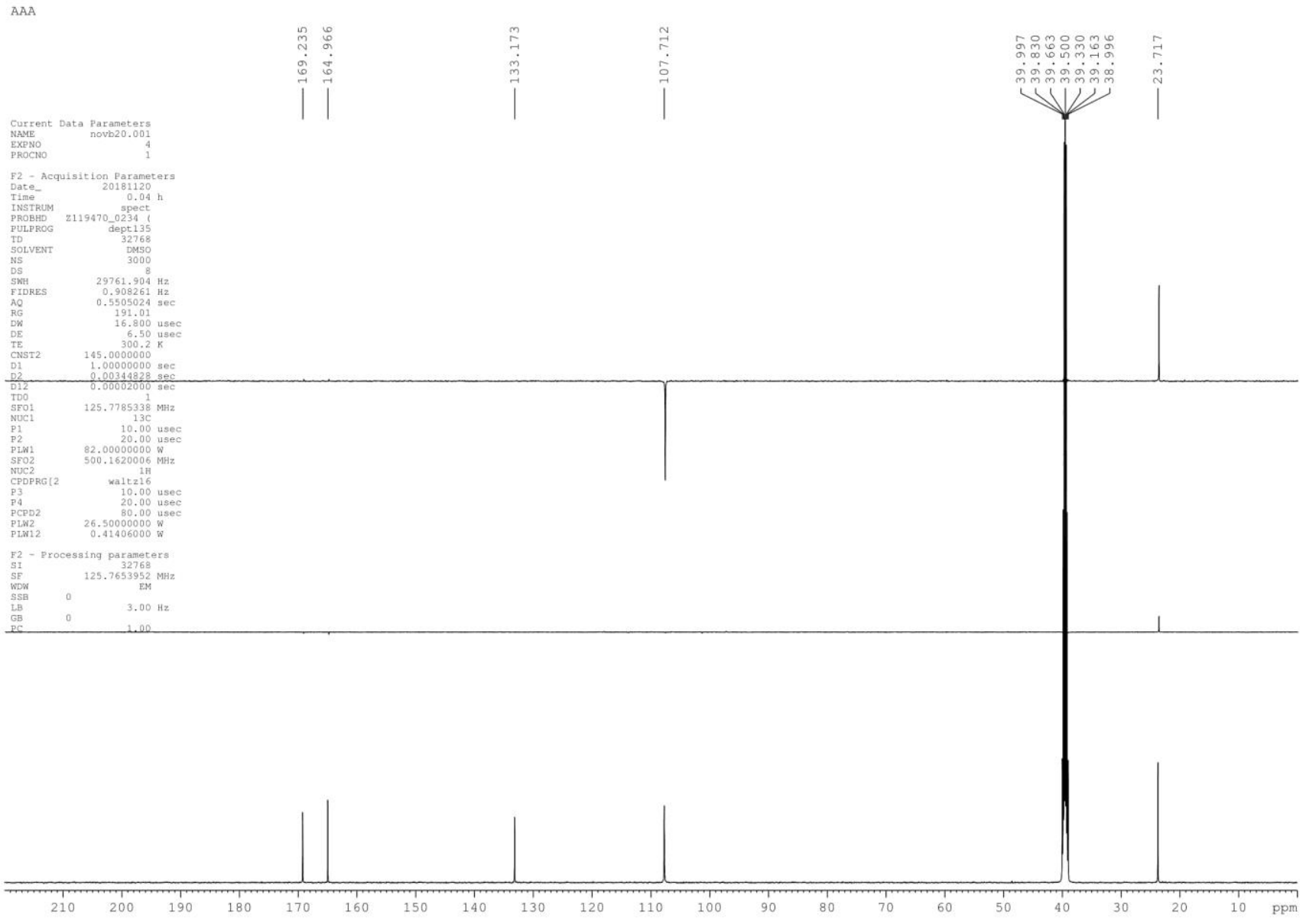

\section{${ }^{13} \mathrm{C}$-DEPT 135 NMR in DMSO-d 6}

2-(acetylamino)acrylic acid 8 


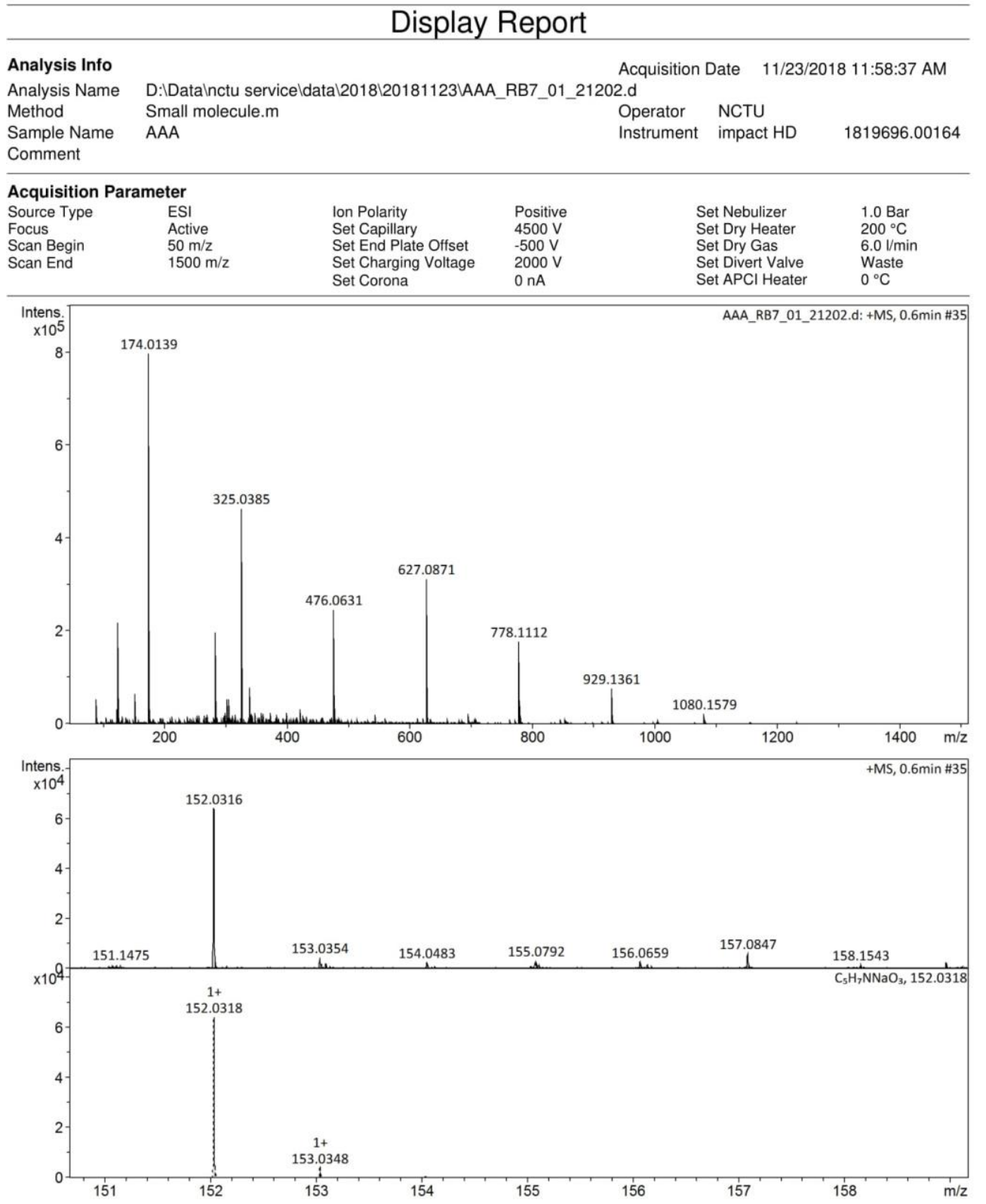

AAA_RB7_01_21202.d

Bruker Compass DataAnalysis 4.1

\section{HR ESI-MS}

2-(acetylamino)acrylic acid 8

Figure 5. Spectra of 2-(acetylamino)acrylic acid 8 
MAA
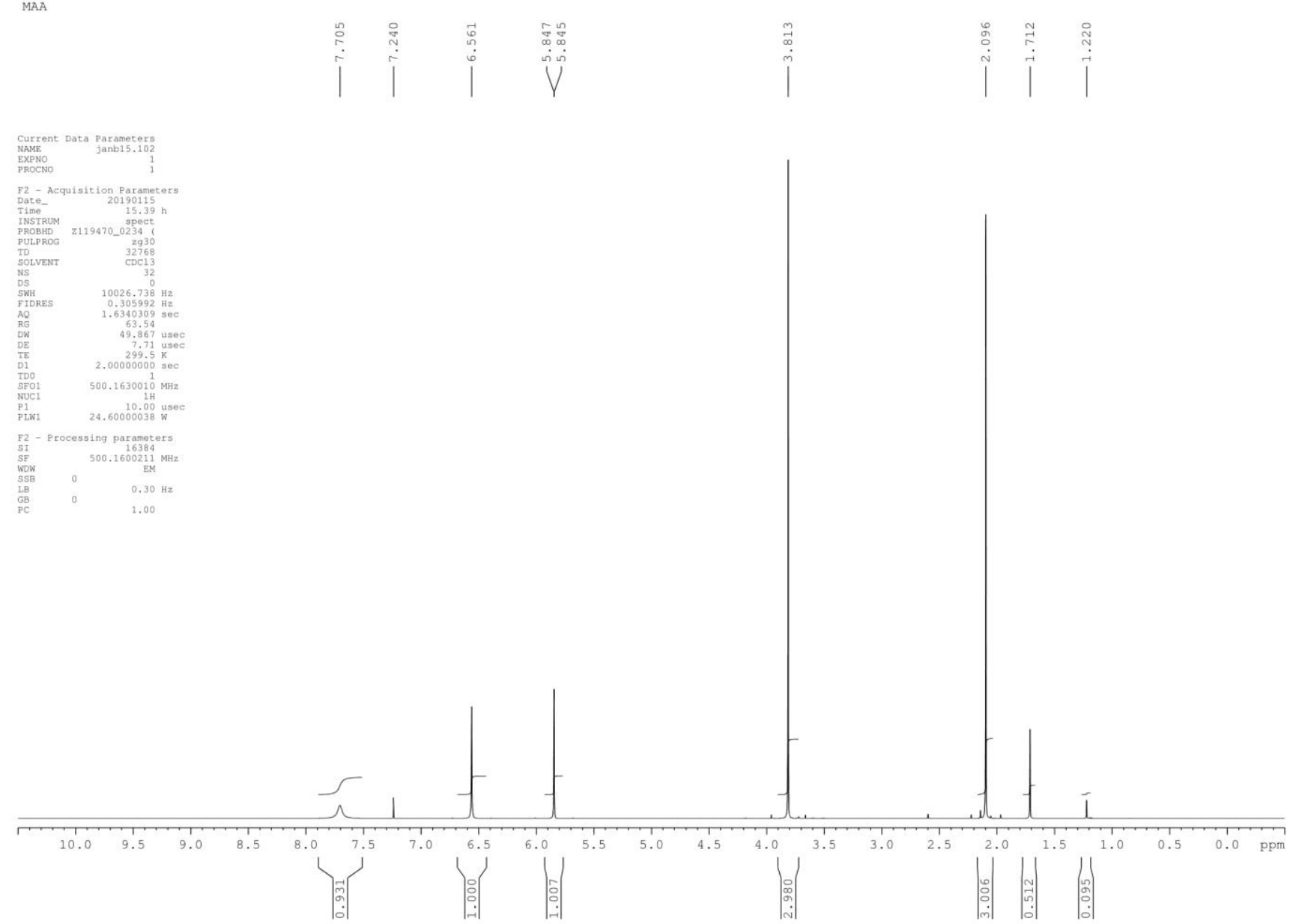

${ }^{1} \mathrm{H}-\mathrm{NMR}$ in $\mathrm{CDCl}_{3}$<smiles>C=C(NC(C)=O)C(=O)OC</smiles>

2-acetamidoacrylate methyl ester 9 

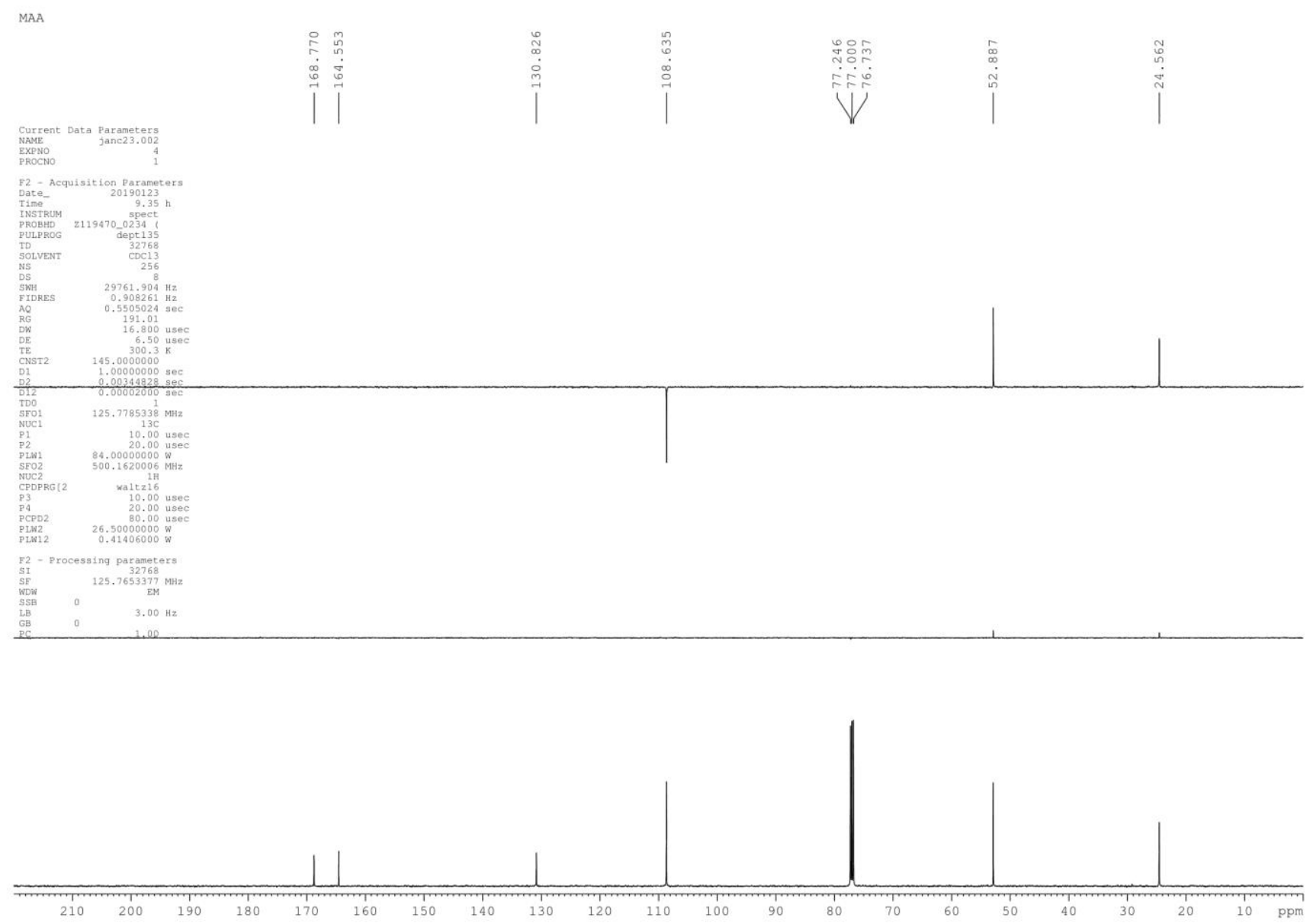

\section{${ }^{13} \mathrm{C}$ DEPT-135 NMR in $\mathrm{CDCl}_{3}$}

2-acetamidoacrylate methyl ester 9 


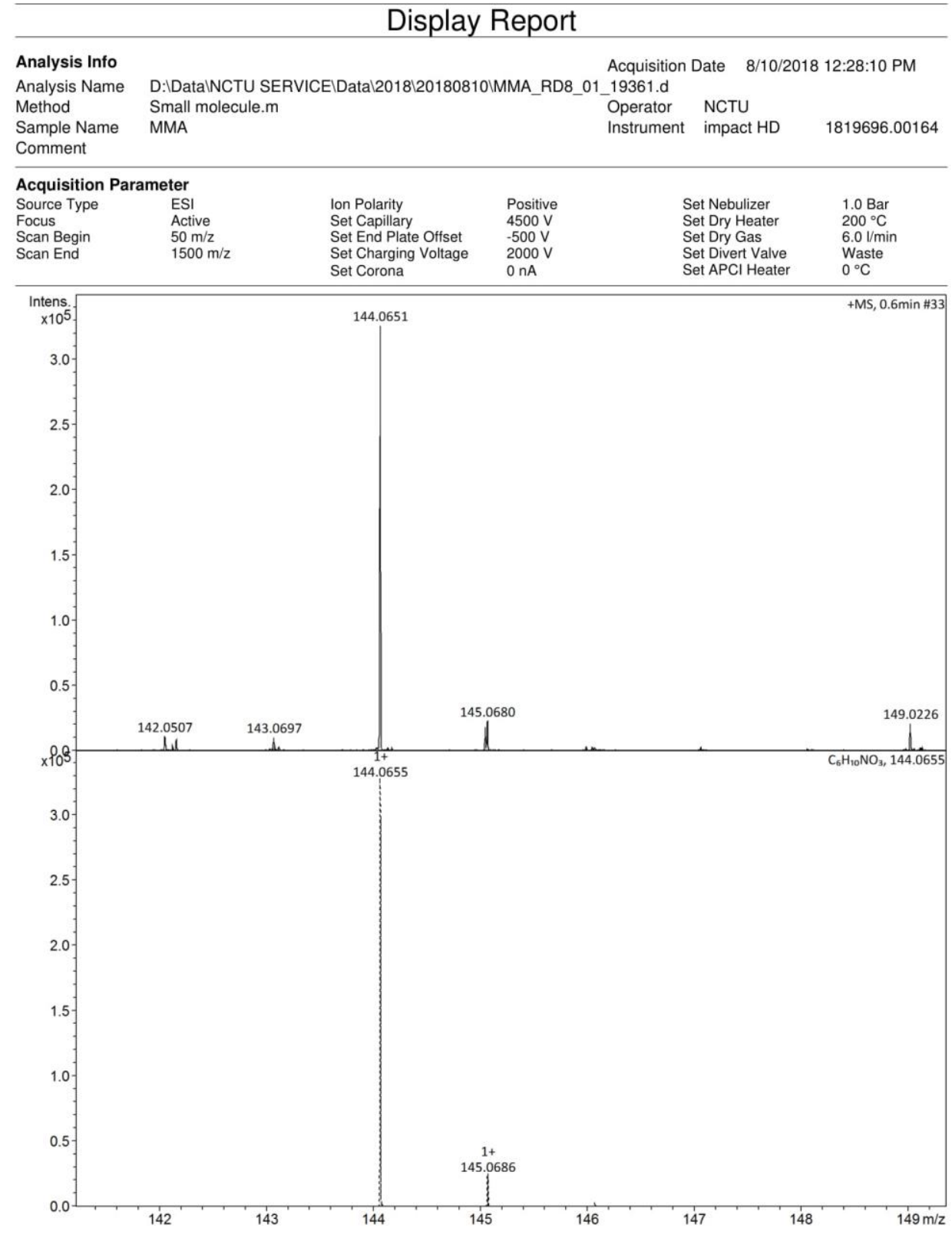

HR ESI-MS

2-acetamidoacrylate methyl ester 9

Figure 6. Spectra of 2-acetamidoacrylate methyl ester 9 


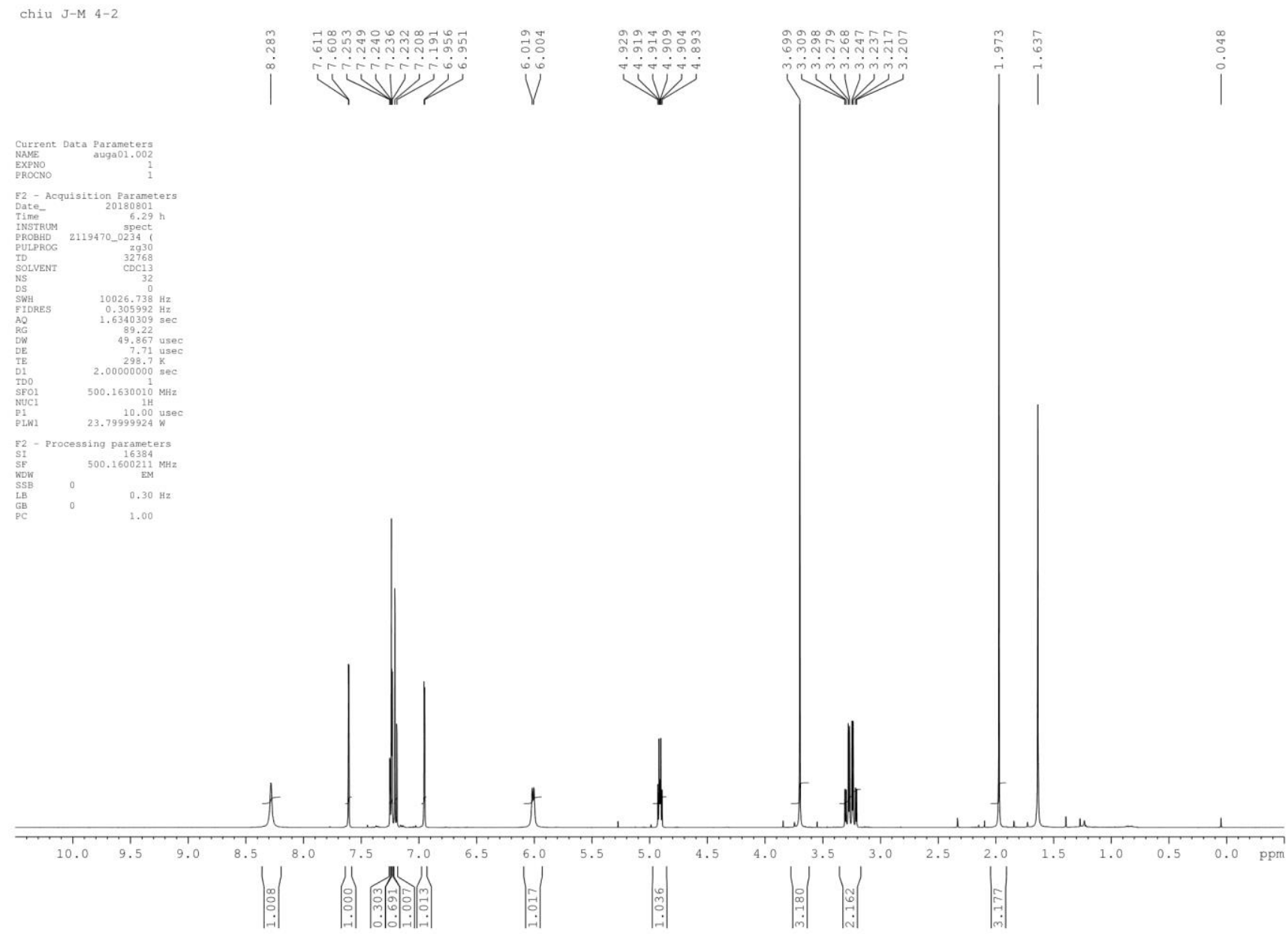

${ }^{1} \mathrm{H}-\mathrm{NMR}$ in $\mathrm{CDCl}_{3}$<smiles>COC(=O)C(Cc1c[nH]c2ccc(Br)cc12)NC(C)=O</smiles>

5-bromo-3-(2R,2S)(2-N-acetyl)tryptophan methyl ester $\mathbf{1 0}$ 


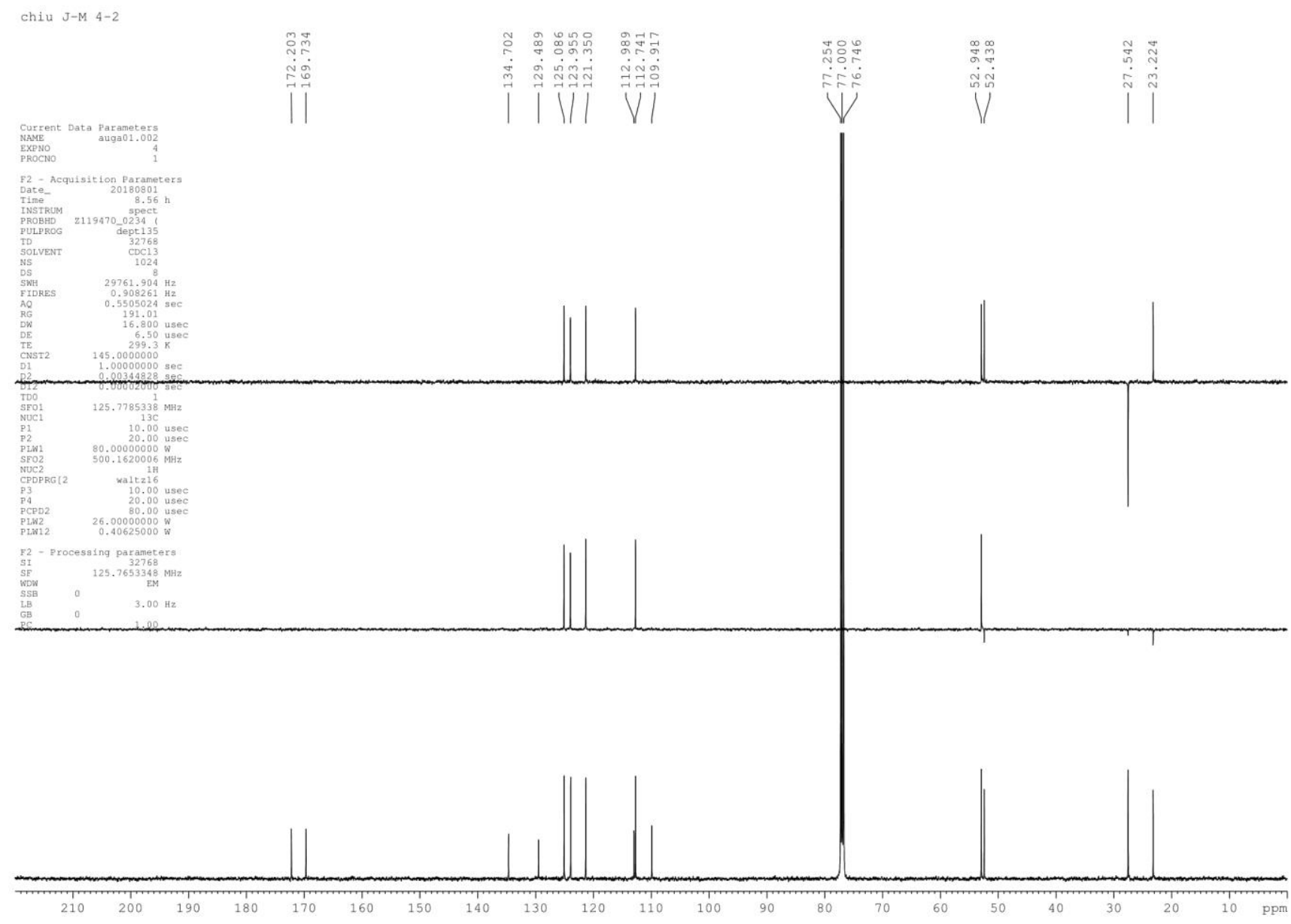

${ }^{13} \mathrm{C}$ DEPT-135 NMR in $\mathrm{CDCl}_{3}$

5-bromo-3-(2R, $2 S)(2-N$-acetyl)tryptophan methyl ester $\mathbf{1 0}$ 


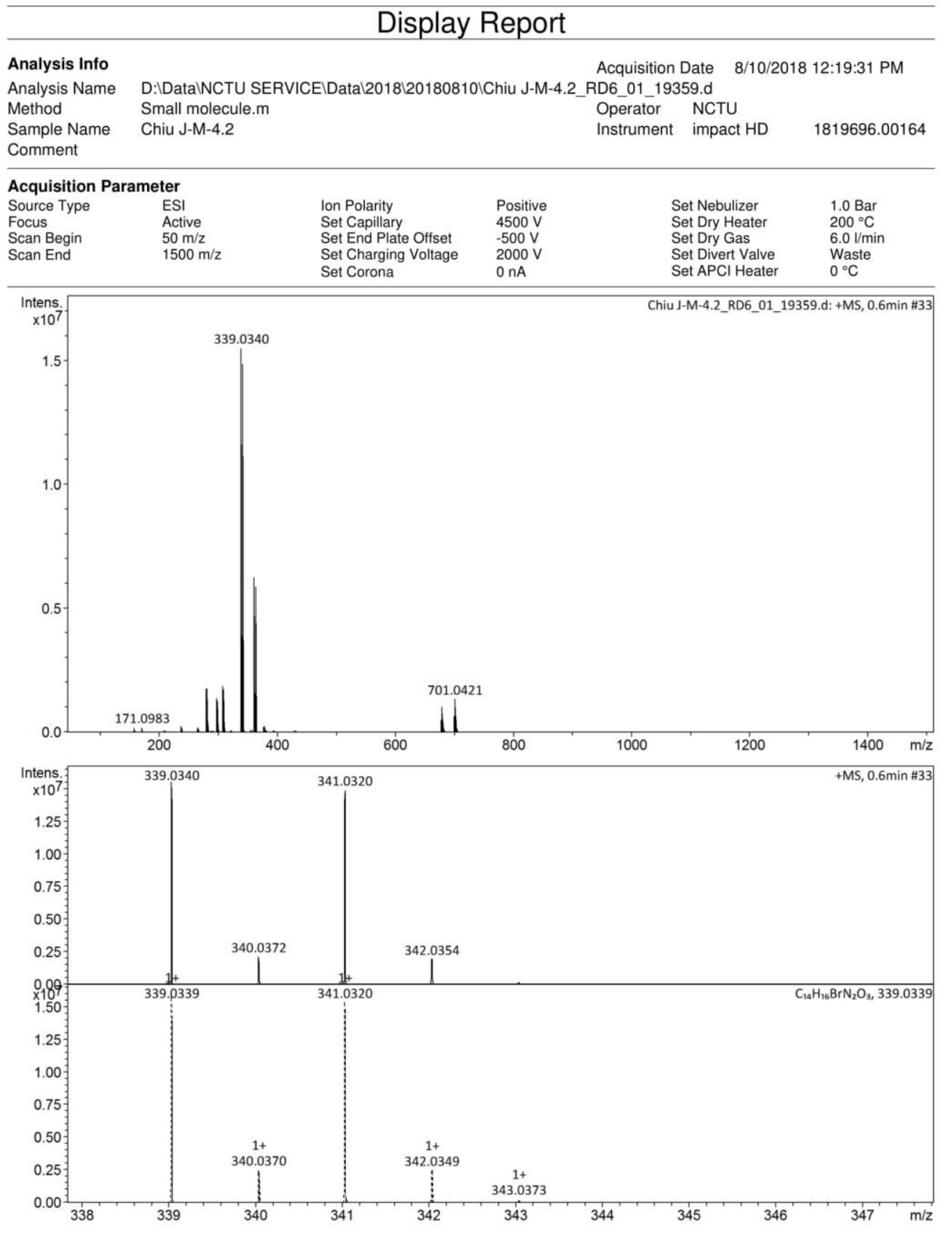

\section{HR ESI-MS}

5-bromo-3-(2R, $2 S)(2-N$-acetyl)tryptophan methyl ester $\mathbf{1 0}$ 


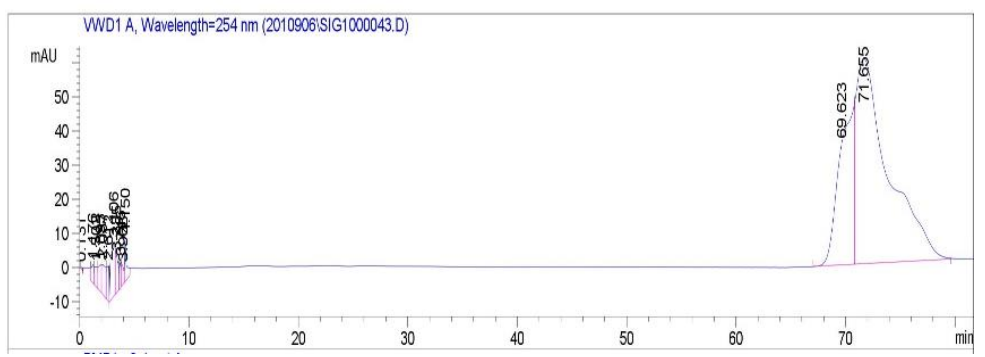

RP-18 Chiral HPLC chromatogram of compound $\mathbf{1 0}$

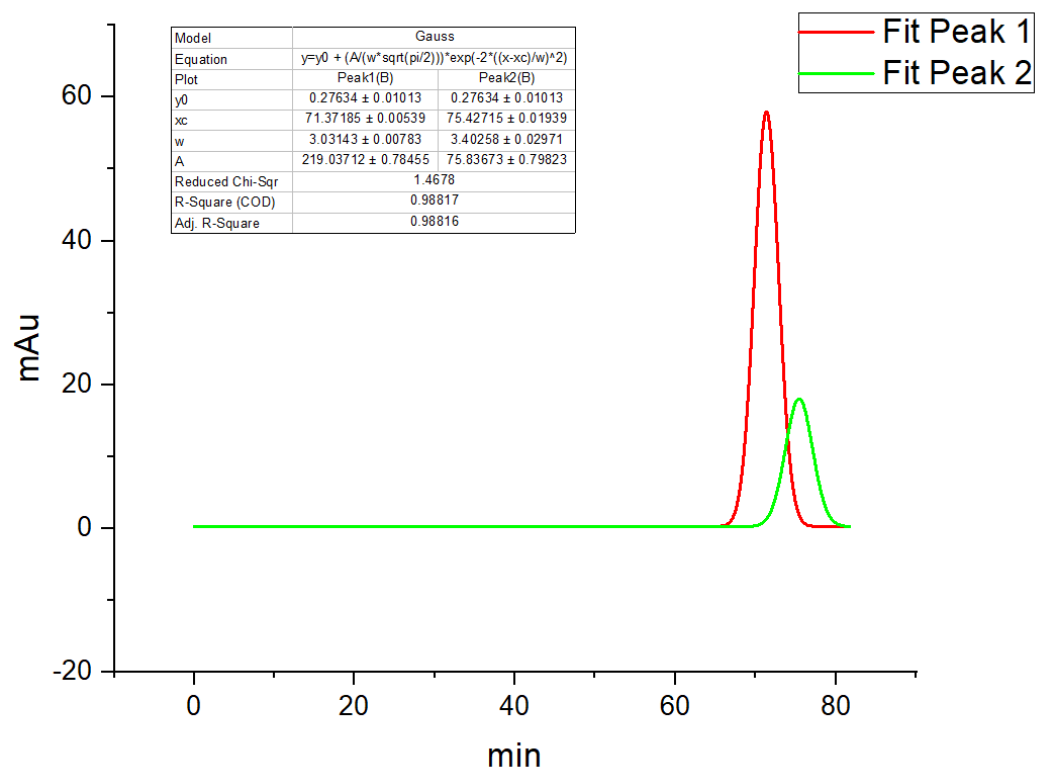

Software Origin-derived two peaks implying two $d, l$ forms

Figure 7. Spectra and chromatogram of 5-bromo-3(2R,2S)-(2-N-acetyl)tryptophan methyl ester 10 


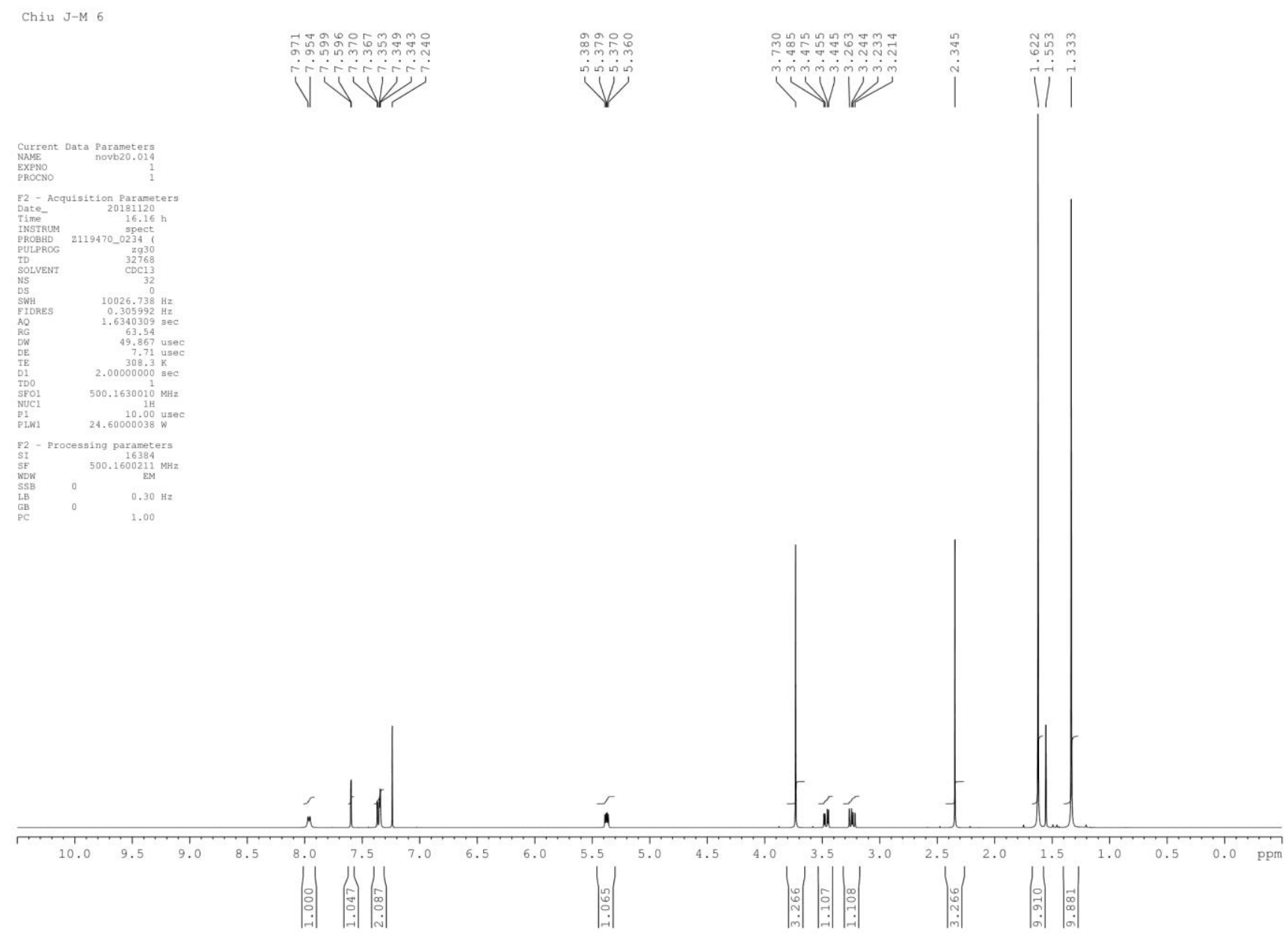

${ }^{1} \mathrm{H}-\mathrm{NMR}$ in $\mathrm{CDCl}_{3}$<smiles>COC(=O)C(Cc1cn(C(=O)OC(C)(C)C)c2ccc(Br)cc12)N(C(C)=O)C(C)=O</smiles>

3-(2R,2S)(2-N-acetyl)-1,N-diBoc-5-bromotryptophan methyl ester 11 


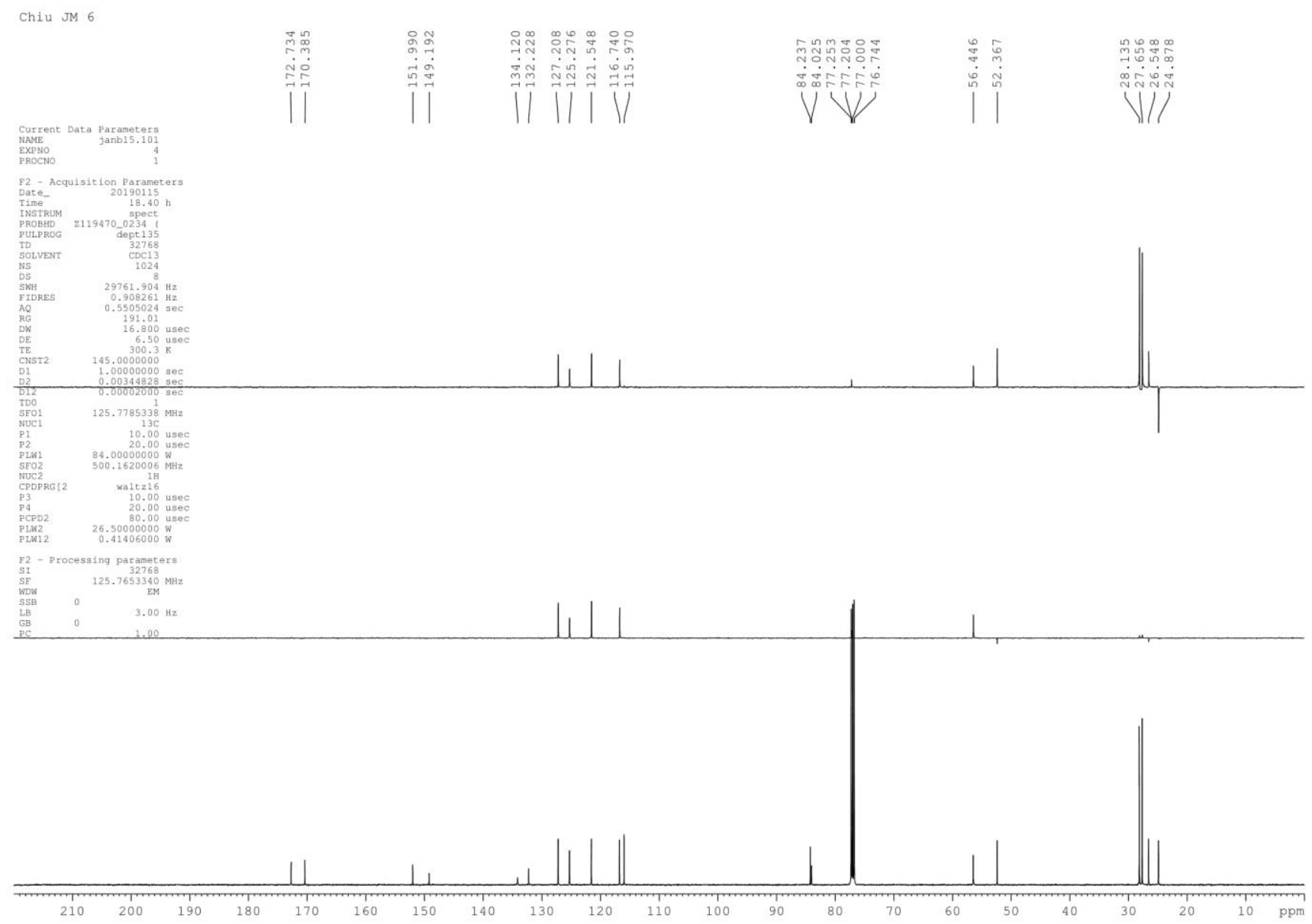

\section{${ }^{13} \mathrm{C}$ DEPT-135 NMR in $\mathrm{CDCl}_{3}$}

3-(2R,2S)(2-N-acetyl)-1,N-diBoc-5-bromotryptophan methyl ester 11 


\section{Display Report}

Analysis Info

Acquisition Date 8/28/2018 10:41:49 AM

Analysis Name D:IDatalnctu serviceldatal2018|20180828|Chiu J-M 6_GD8_01_19681.d

Method Small molecule.m $\quad$ Operato

Instrument impact HD

1819696.00164

Comment

Acquisition Paramete

Source Type ESI

Focus

Scan Begin $\quad 50 \mathrm{~m} / 2$

Scan End $\quad 1500 \mathrm{~m} / 2$

Ion Polarity

Set End Plate Offset

Set Charging Voltage

Set Corona

Positive Set Nebulizer $1.0 \mathrm{Bar}$

$-500 \mathrm{~V}$

Set Dry Heater $\quad 200^{\circ} \mathrm{C}$

Set Dry Gas $\quad 6.01 / \mathrm{min}$

$0 \mathrm{nA}$

Set APCI Heater

Waste
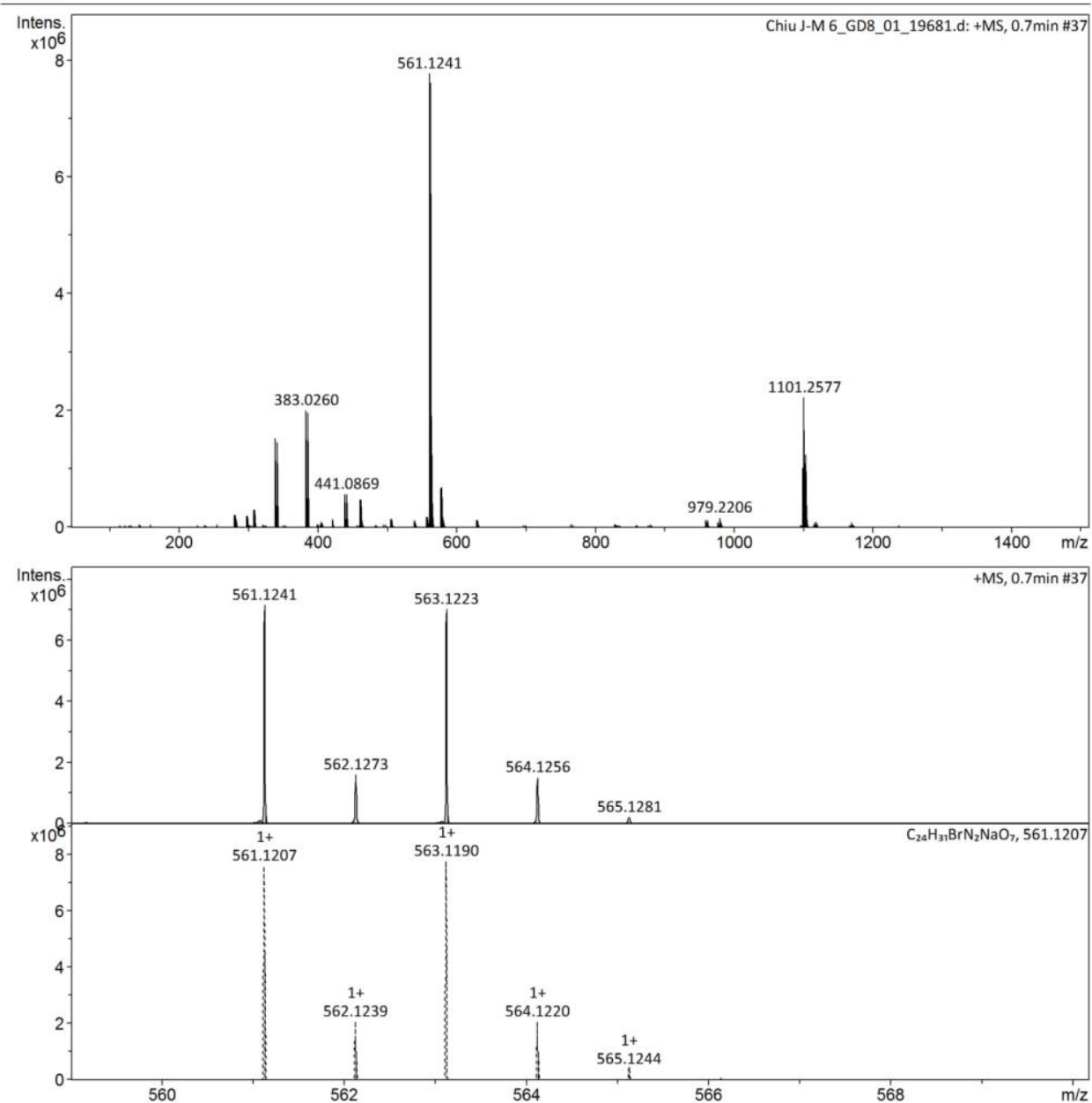


\section{HR ESI-MS}

3-(2R,2S)(2-N-acetyl)-1,N-diBoc-5-bromotryptophan methyl ester 11

Figure 8. Spectra of 3-(2R,2S)(2-N-acetyl)-1,N-diBoc-5-bromotryptophan methyl ester 11

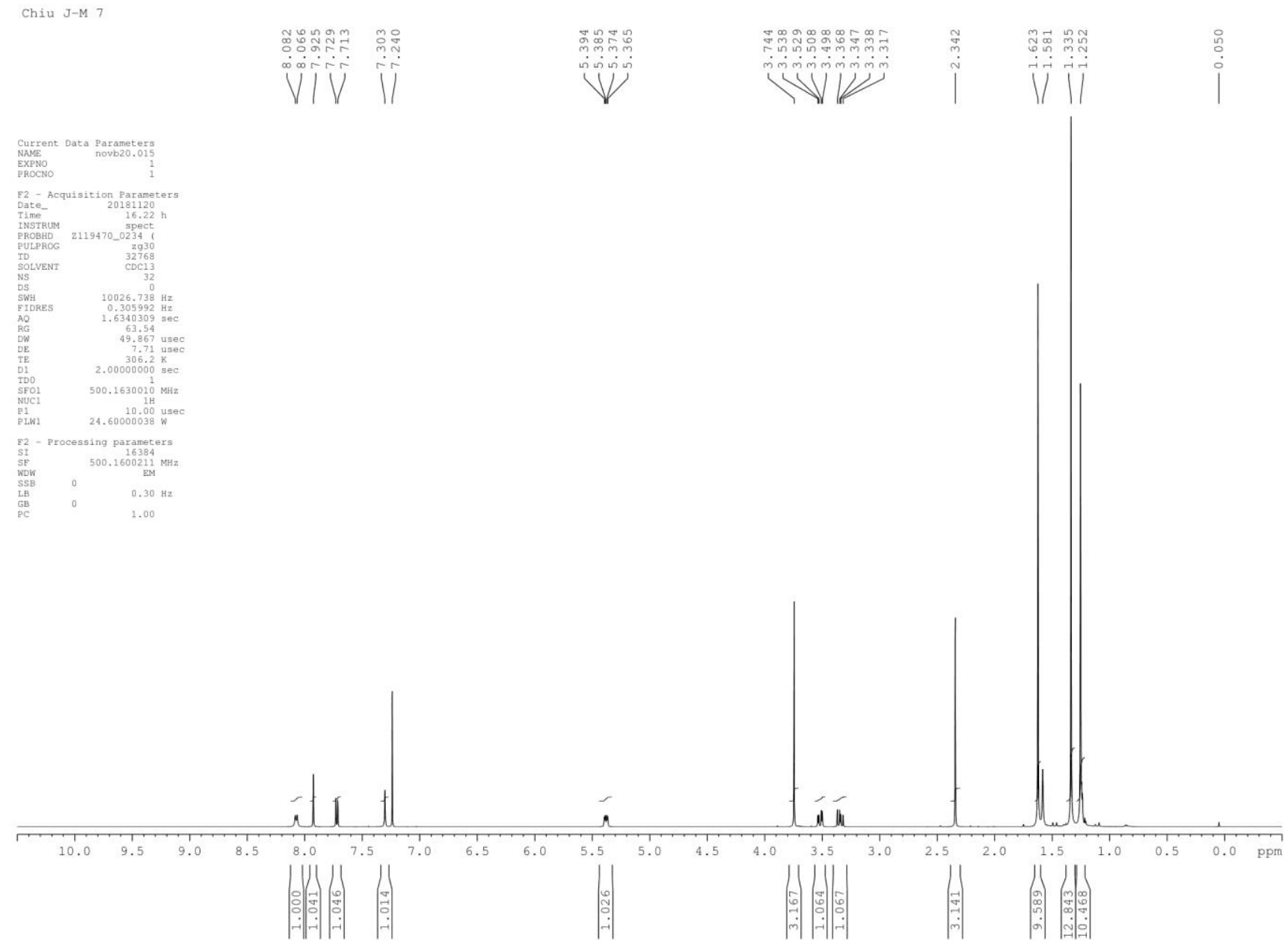

${ }^{1} \mathrm{H}-\mathrm{NMR}$ in $\mathrm{CDCl}_{3}$<smiles>COC(=O)C(Cc1cn(C(=O)OCc2ccccc2)c2ccc(B3OC(C)(C)C(C)(C)O3)cc12)N(C(C)=O)C(C)=O</smiles>

DBA-5-BT 2 


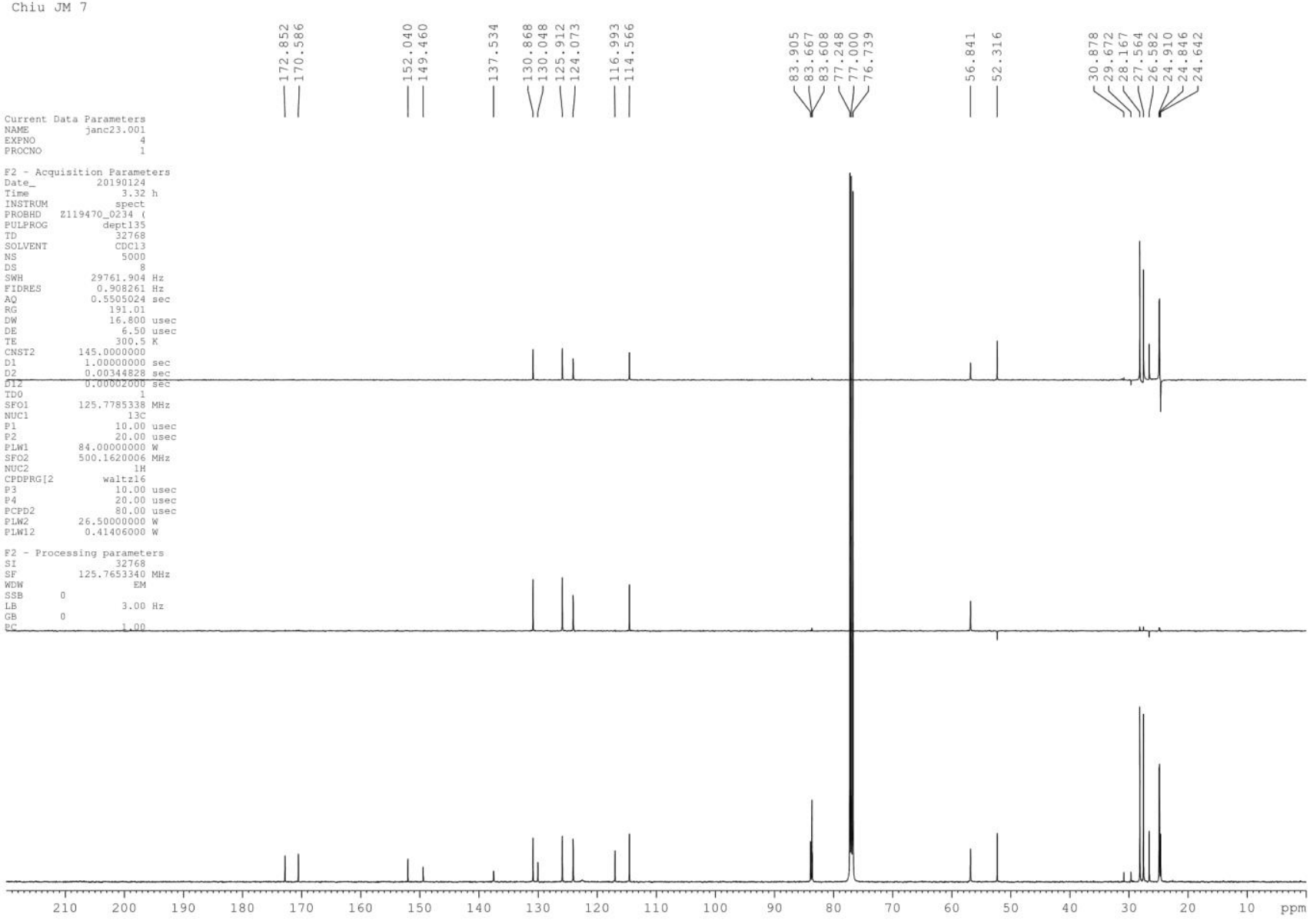

\section{${ }^{13} \mathrm{C}$ DEPT-135 NMR in $\mathrm{CDCl}_{3}$}

DBA-5-BT 2 


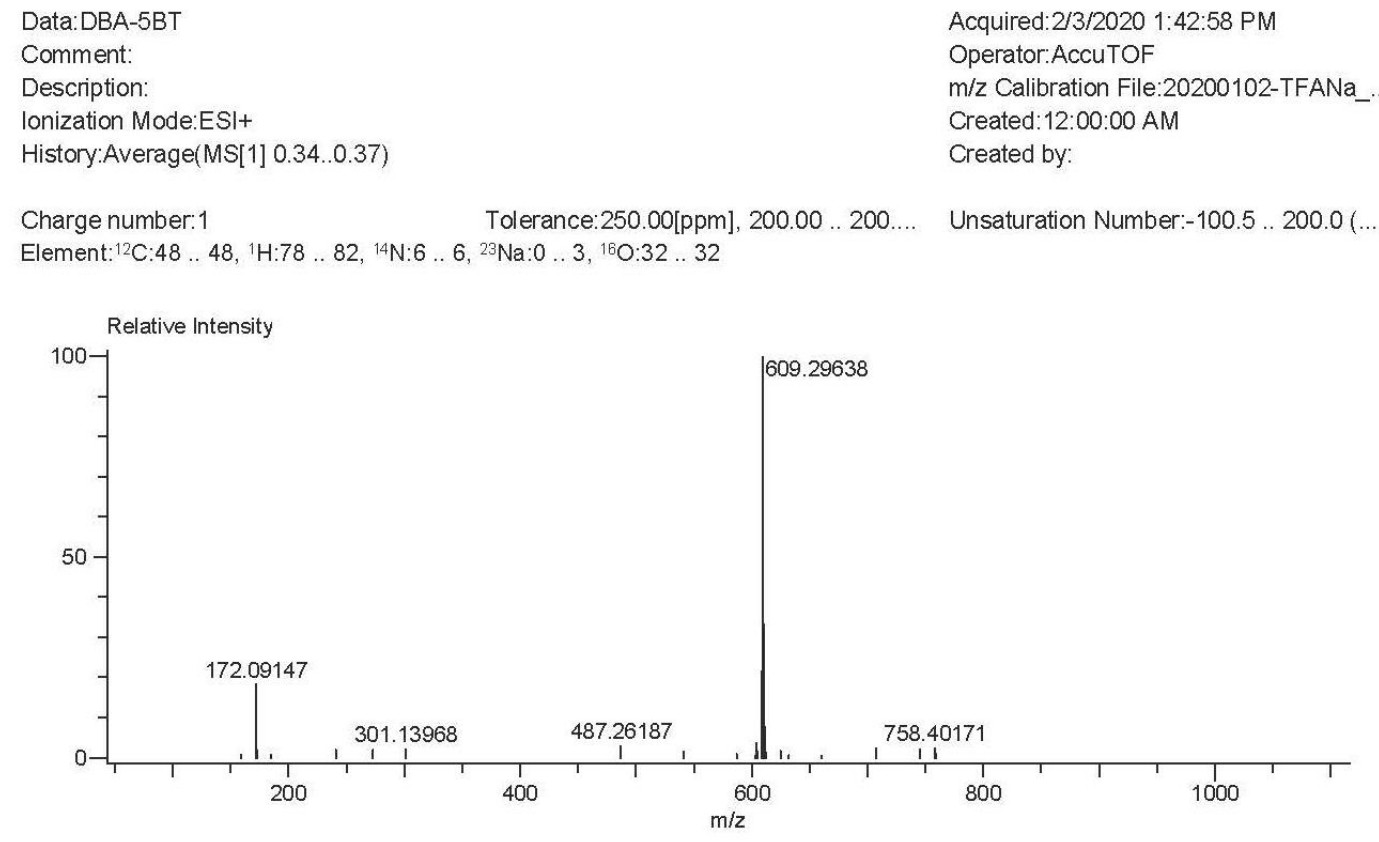

\section{HR ESI-MS}

\section{DBA-5-BT 2}

Figure 9. Spectra of 3-(2R,2S)(2-N-acetyl)-1, $N$-diBoc-5-boronopinacoltryptophan methyl ester (DBA-5-BT) 


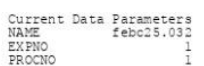

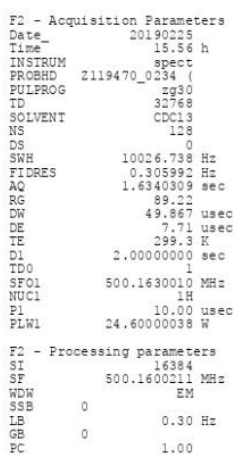

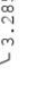

:

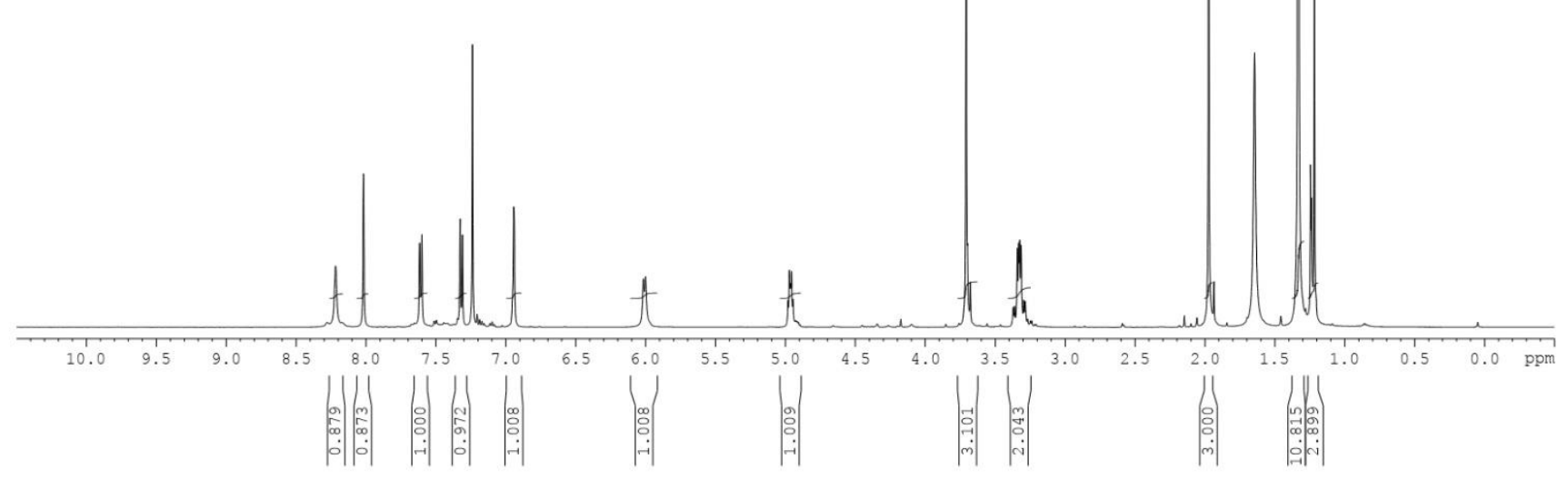

${ }^{1} \mathrm{H}-\mathrm{NMR}$ in $\mathrm{CDCl}_{3}$<smiles>COC(=O)C(Cc1c[nH]c2ccc(B3OC(C)(C)C(C)(C)O3)cc12)NC(C)=O</smiles>

3-(2-N-acetyl)-5-boronopinacoltryptophan methyl ester (NA-5-BT) 3 


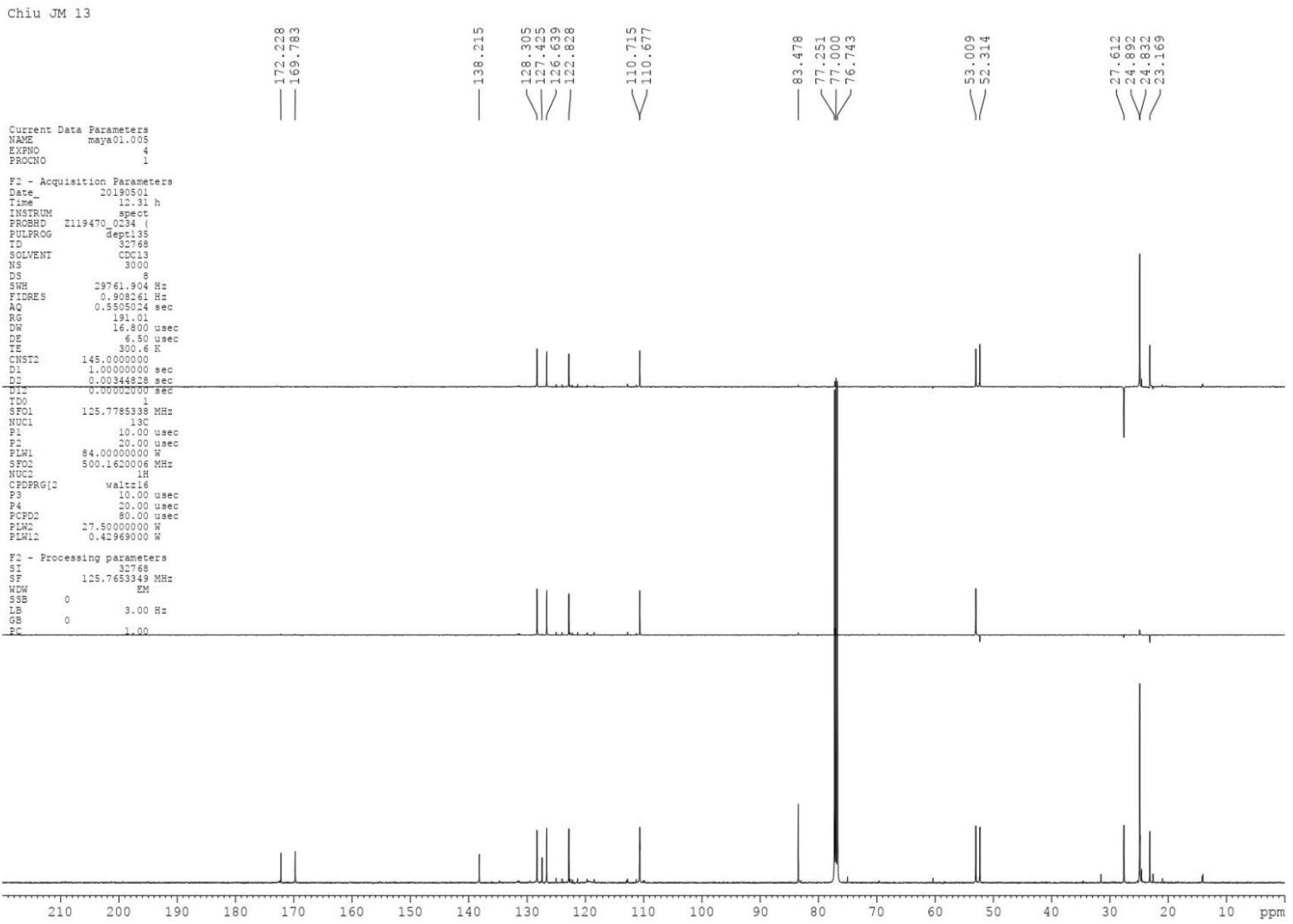

${ }^{13} \mathrm{C}$-DEPT $135 \mathrm{NMR}$ in $\mathrm{CDCl}_{3}$

NA-5-BT 3 
Display Report

Analysis Info

Analysis Name

Method

Sample Name

Comment
Acquisition Date 2/27/2019 11:50:37 AM

D:IDatalNCTU SERVICEIDatal2019|20190227/CHIU JM 13_BB5_01_22501.d

Small molecule.m

CHIU JM 13

Operator NCTU

Instrument impact HD 1819696.00164
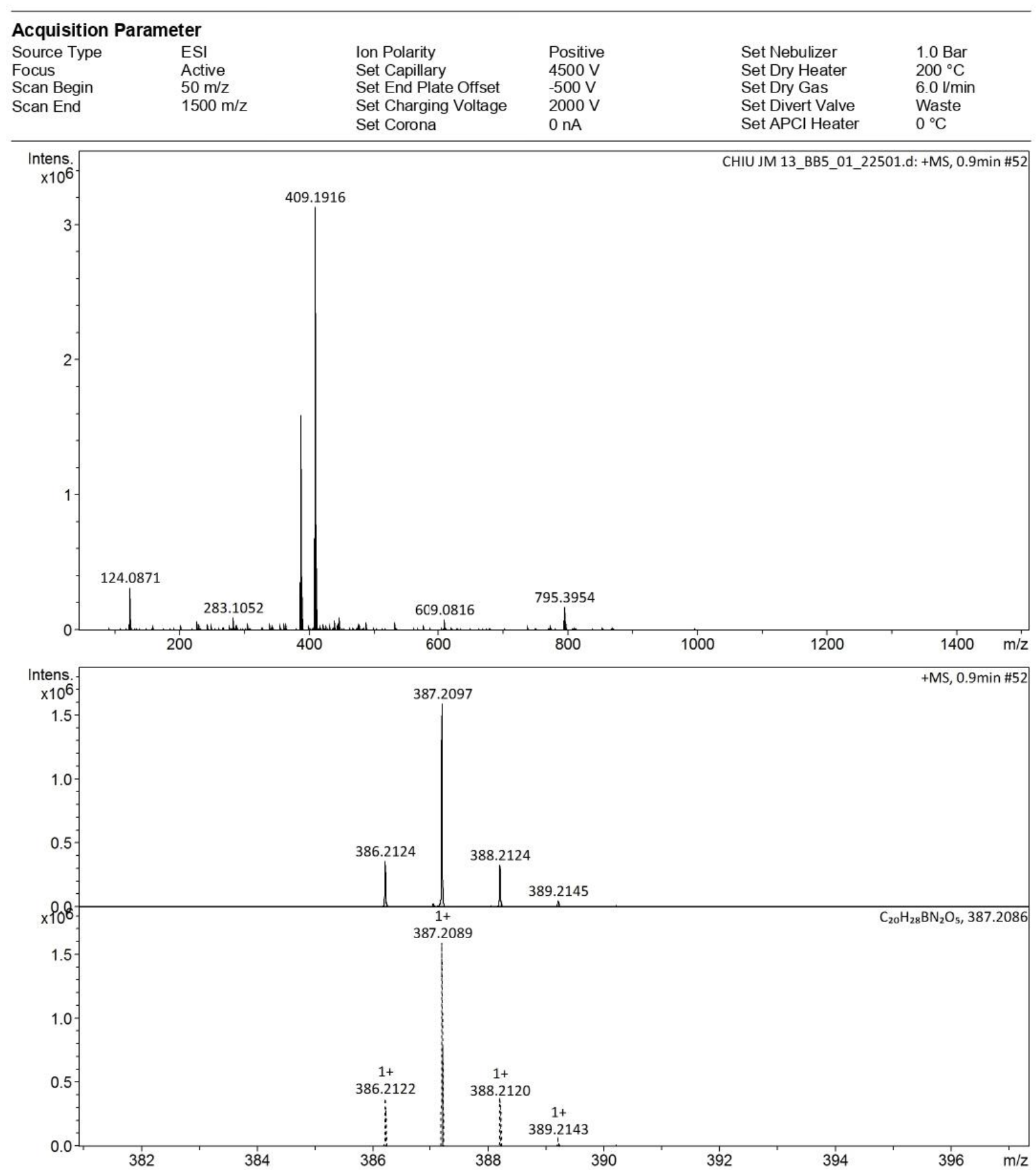

HR ESI-MS

NA-5-BT 3

Figure 10. Spectra of 3-(2- $N$-acetyl)-5-boronopinacoltryptophan methyl ester (NA-5-BT) 3 (NA-5-BT) 3 

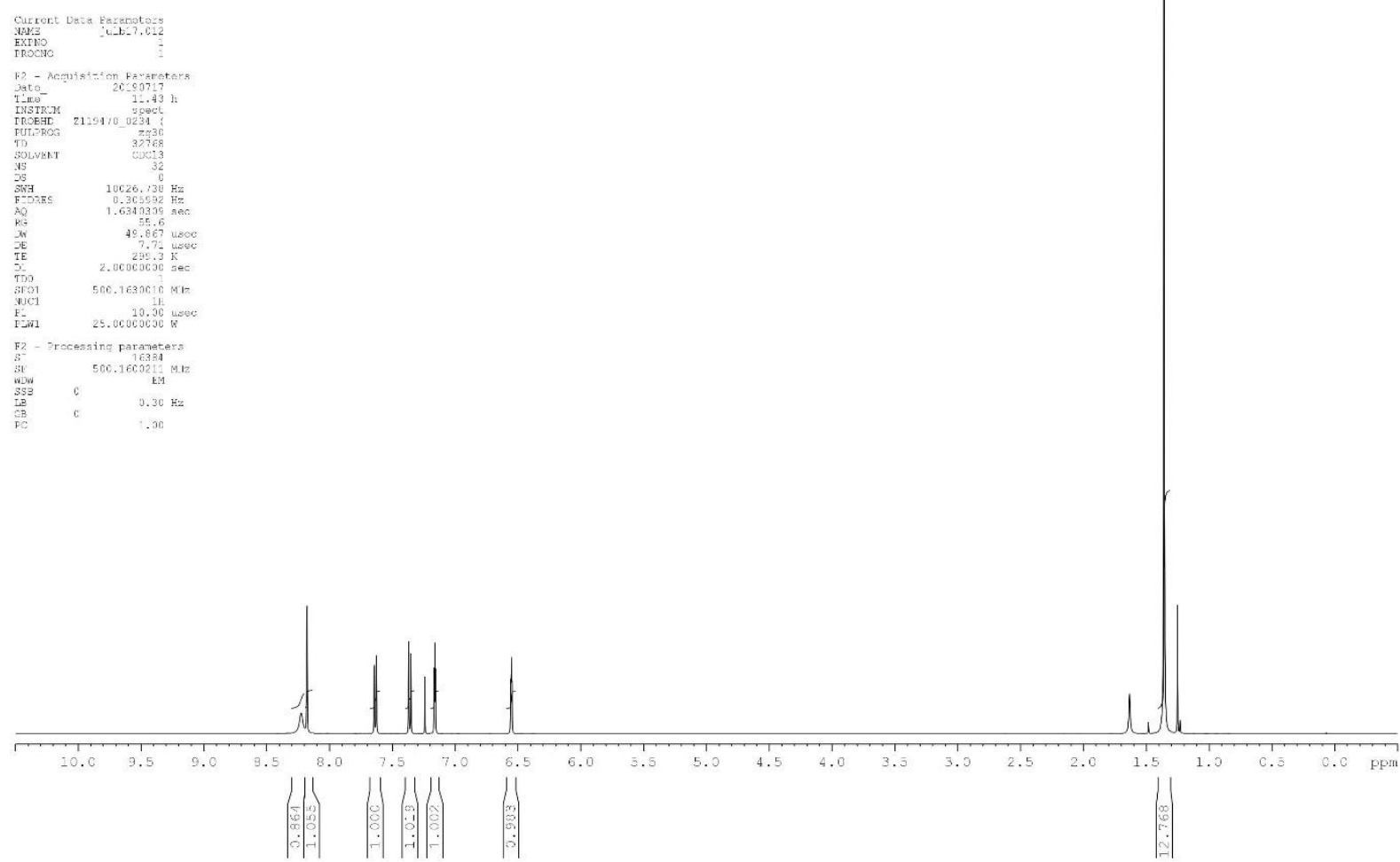<smiles>CC1(C)OB(c2ccc3[nH]ccc3c2)OC1(C)C</smiles>

${ }^{1} \mathrm{H}-\mathrm{NMR}$ in $\mathrm{CDCl}_{3}$

5-Boronopinacol indole 


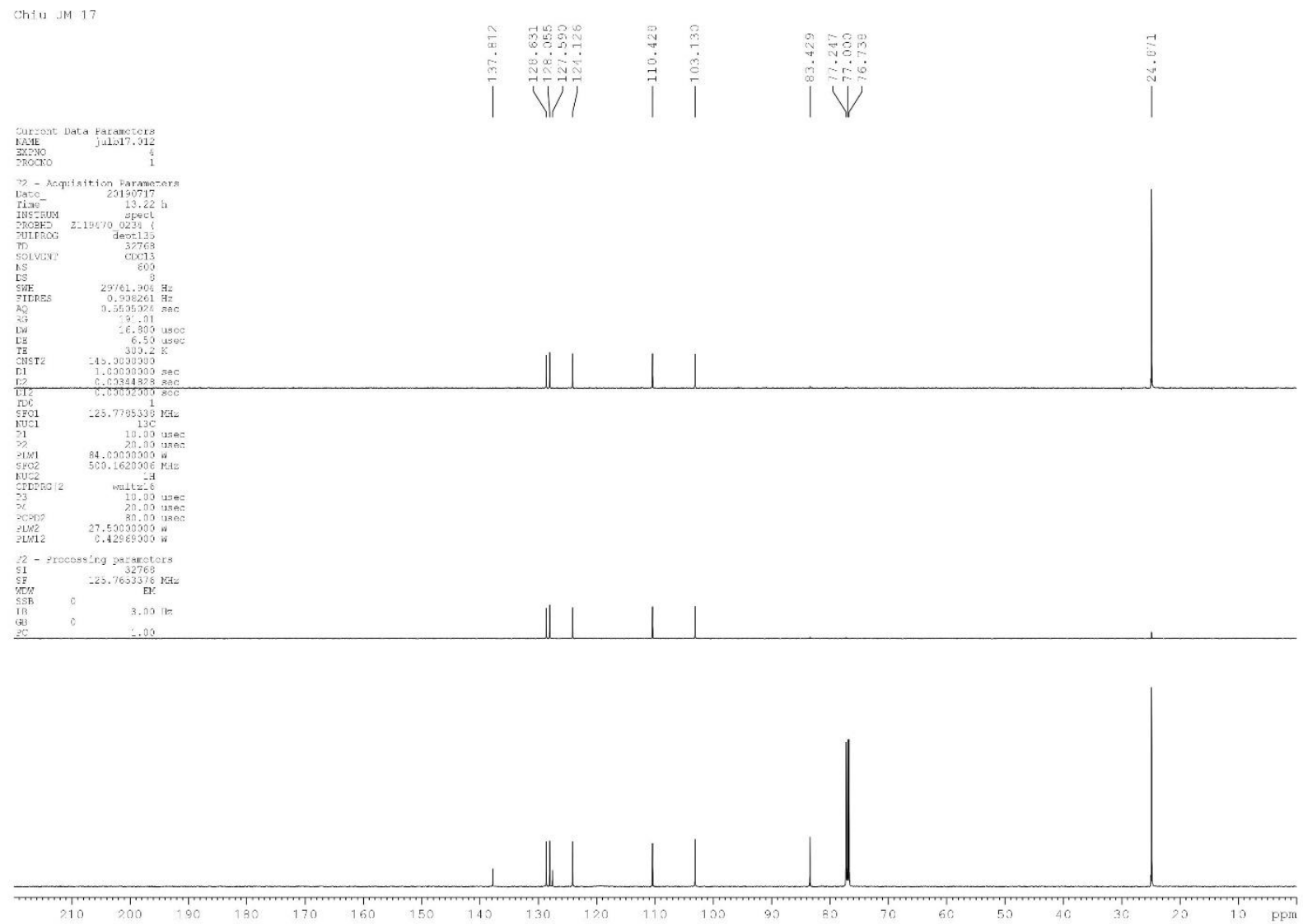

\section{${ }^{13} \mathrm{C}$-DEPT $135 \mathrm{NMR}$ in $\mathrm{CDCl}_{3}$}

5-Boronopinacol indole 


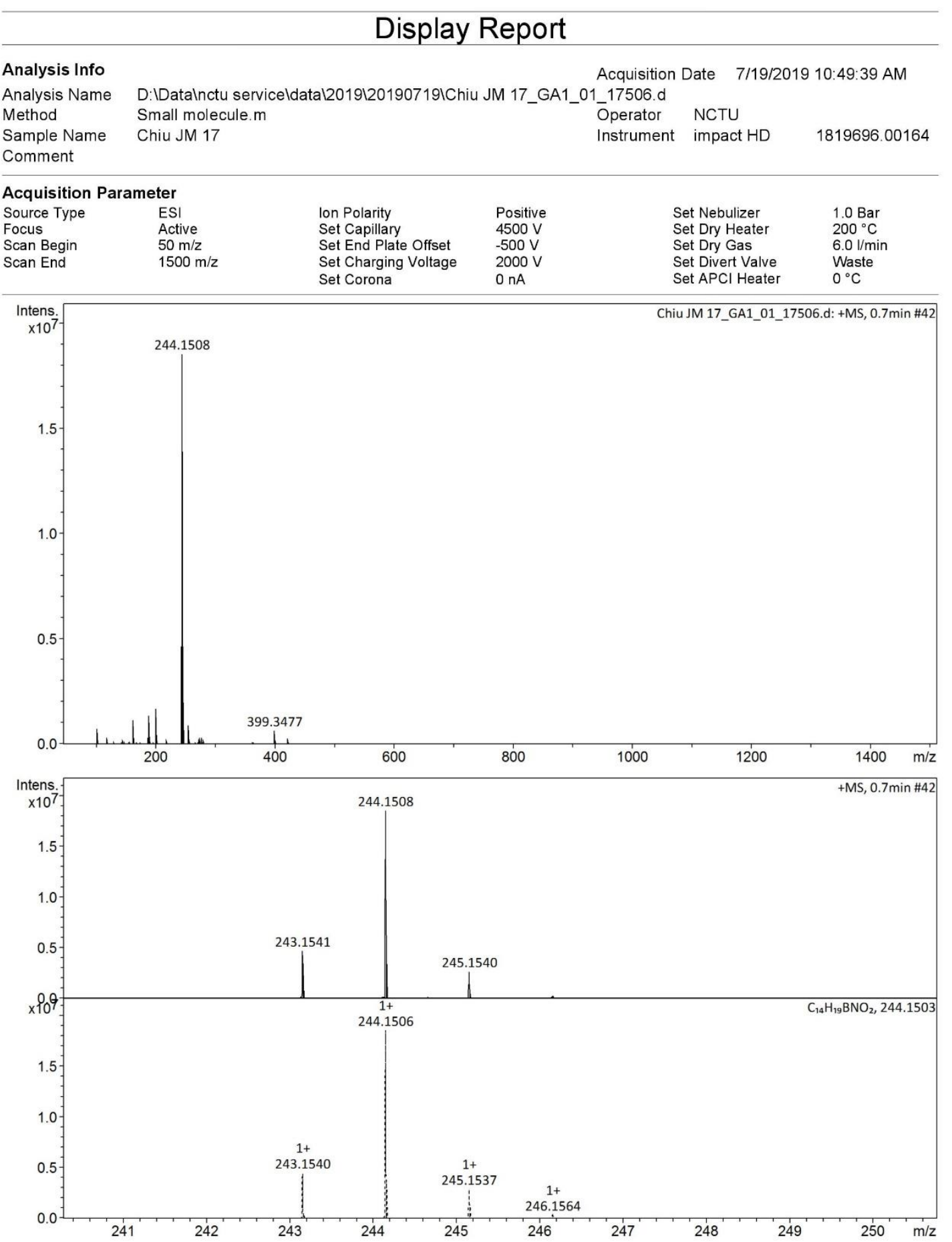

\section{HR ESI-MS}

Figure 11. Spectra of 5-bronopinacol indole 
rIx-

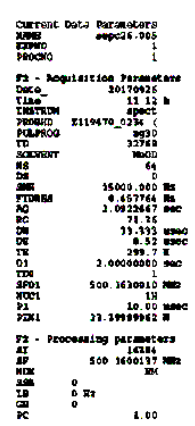

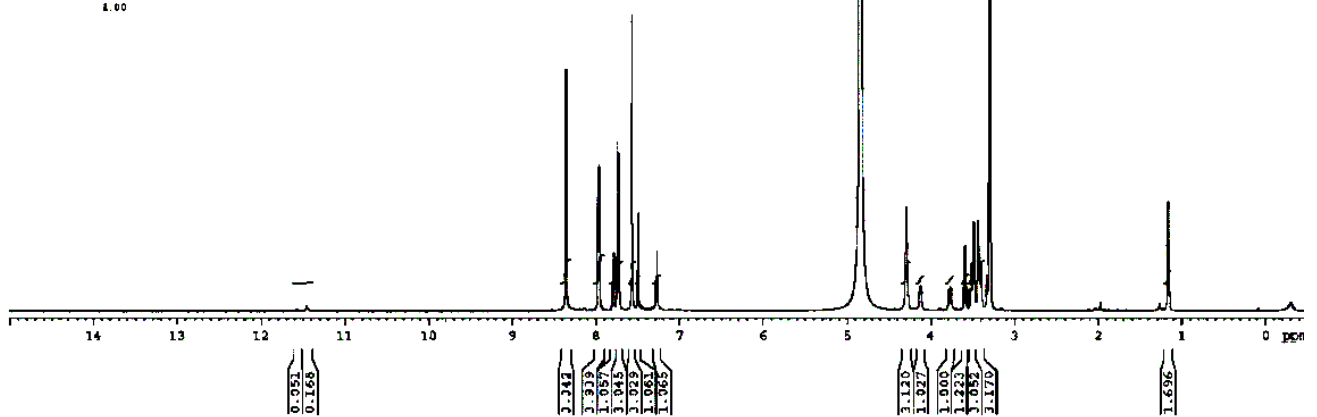<smiles>[R]c1cc([R2])c2c(CC(N)C(=O)O)c[nH]c2c1</smiles>

p- $12 \mathrm{R}_{1}=\mathrm{NO}_{2}, \mathrm{R}_{2}=\mathrm{H}$

o- $12 \mathrm{R}_{1}=\mathrm{H}, \mathrm{R}_{2}=\mathrm{NO}_{2}$

p-12: o-12 = $3: 1$

\section{${ }^{1} \mathrm{H}-\mathrm{NMR}$ in $\mathrm{CD}_{3} \mathrm{OD}$}

6-nitro tryptophan $\boldsymbol{p}-\mathbf{1 2}$ and 4-nitro tryptophan $\boldsymbol{o - 1 2}$ 


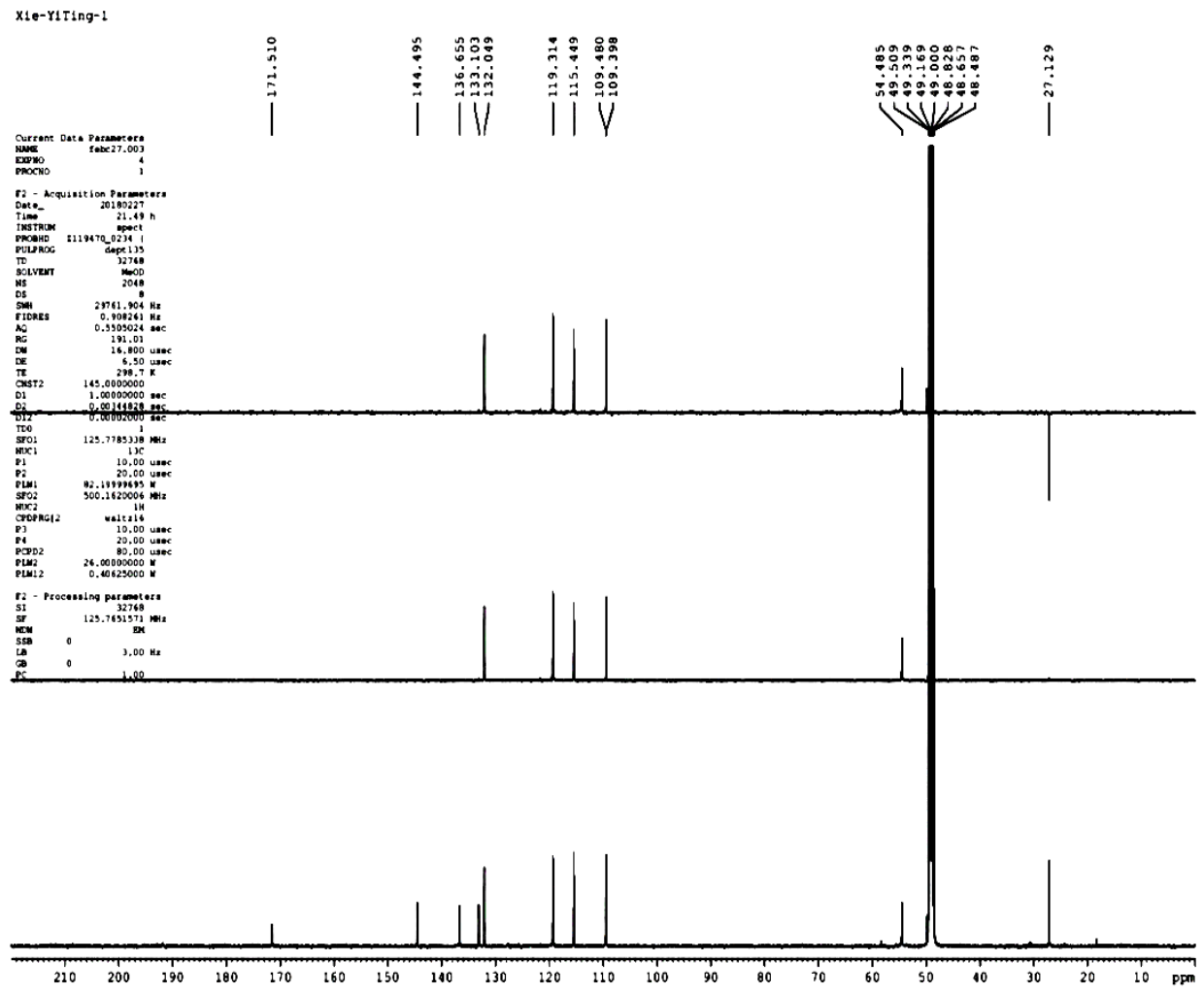

\section{${ }^{13} \mathrm{C}$-DEPT $135 \mathrm{NMR}$ in $\mathrm{CD}_{3} \mathrm{OD}$}

6-nitro tryptophan $\boldsymbol{p}-\mathbf{1 2}$ and 4-nitro tryptophan $\boldsymbol{o - 1 2}$ 
Display Report

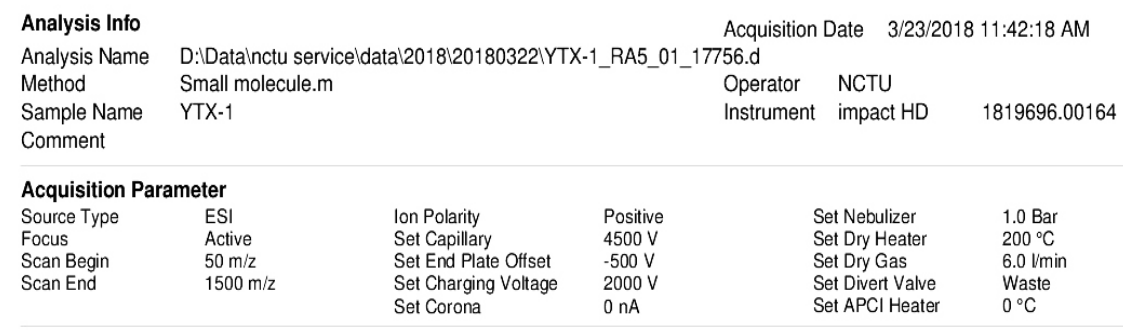
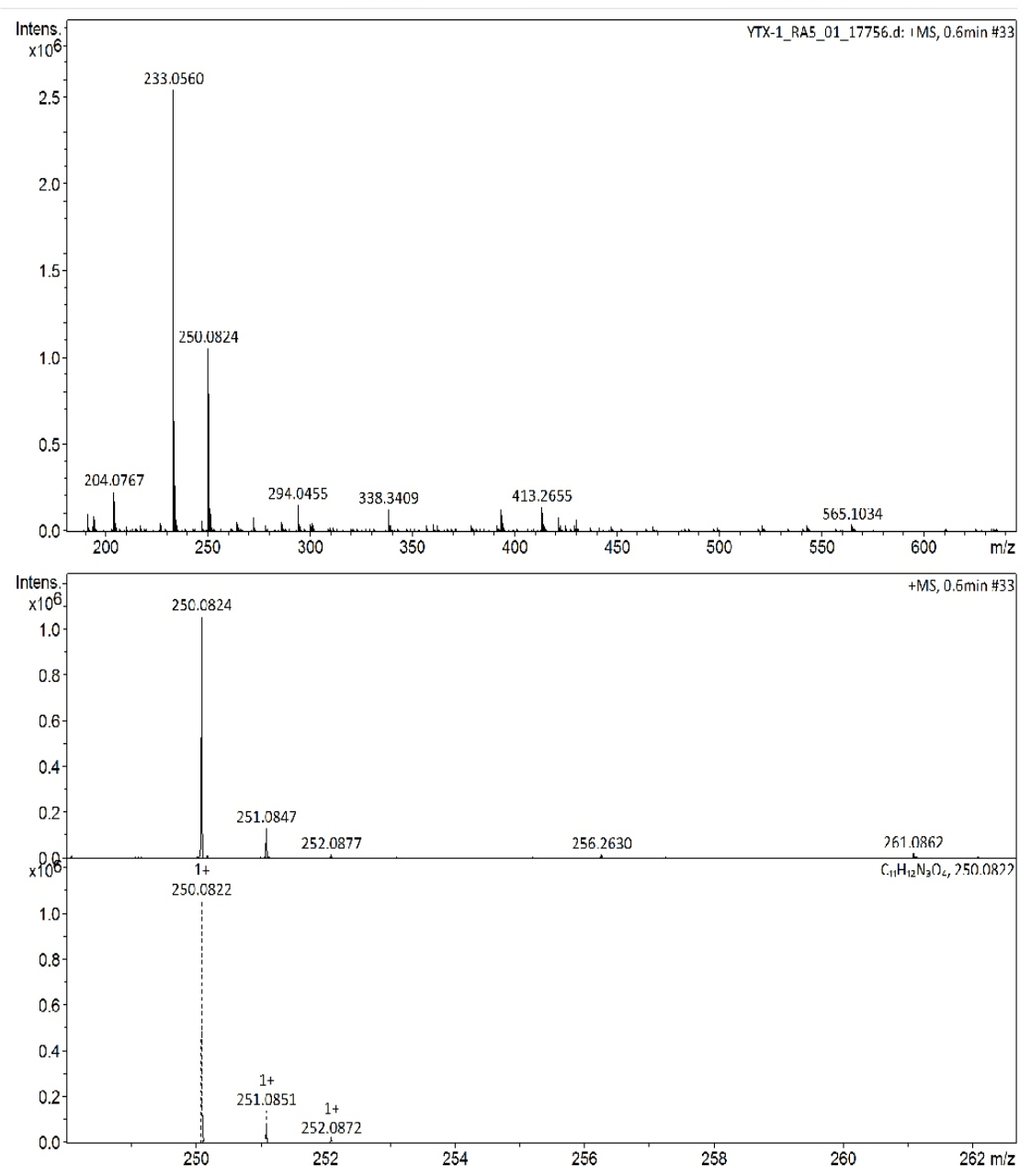

HR ESI-MS

6-nitro tryptophan $\boldsymbol{p - 1 2}$ and 4-nitro tryptophan $\boldsymbol{o - 1 2}$

Figure 12. Spectra of 6-nitro tryptophan $\boldsymbol{p - 1 2}$ and 4-nitro tryptophan $\boldsymbol{o - 1 2}$ 


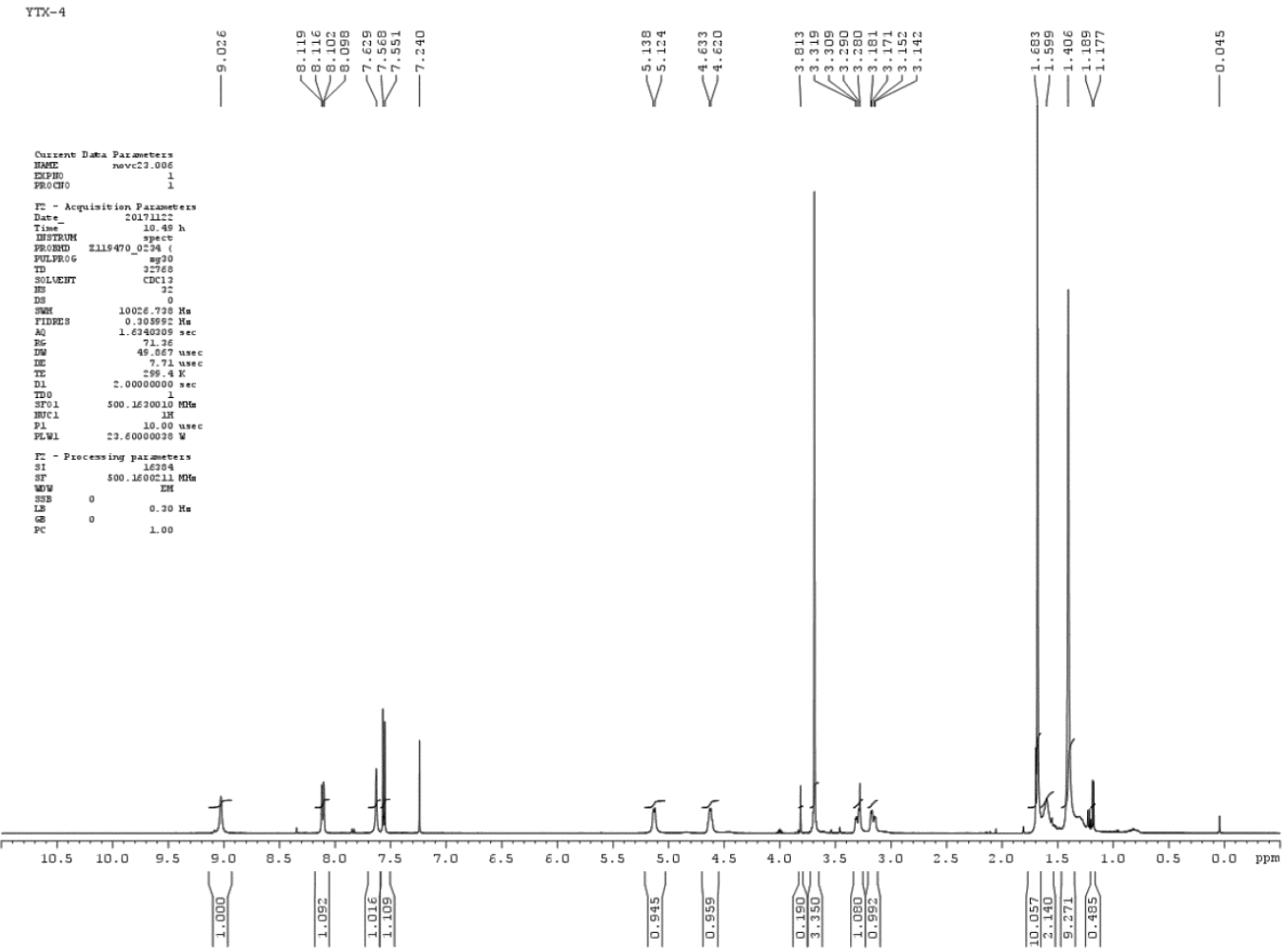

${ }^{1} \mathrm{H}-\mathrm{NMR}$ in $\mathrm{CDCl}_{3}$<smiles>COC(=O)c1ccc([N+](=O)[O-])cc1</smiles>

1,3-(2-N)diBoc-6-nitro tryptophan methyl ester $\boldsymbol{p}$-13 


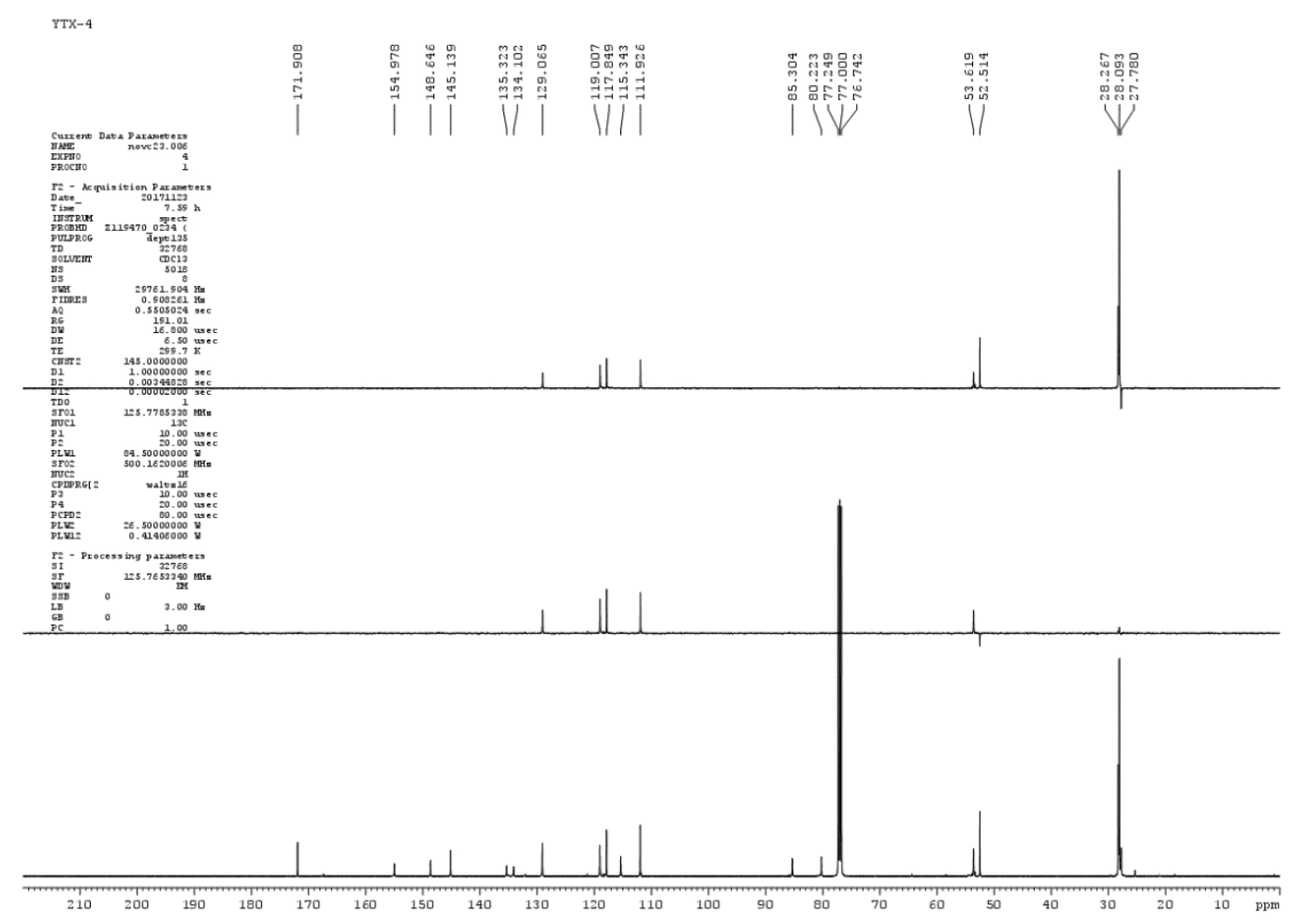

${ }^{13} \mathrm{C}$-DEPT $135 \mathrm{NMR}$ in $\mathrm{CDCl}_{3}$

1,3-(2-N)diBoc-6-nitro tryptophan methyl ester $\boldsymbol{p}-\mathbf{1 3}$ 


\section{Display Report}

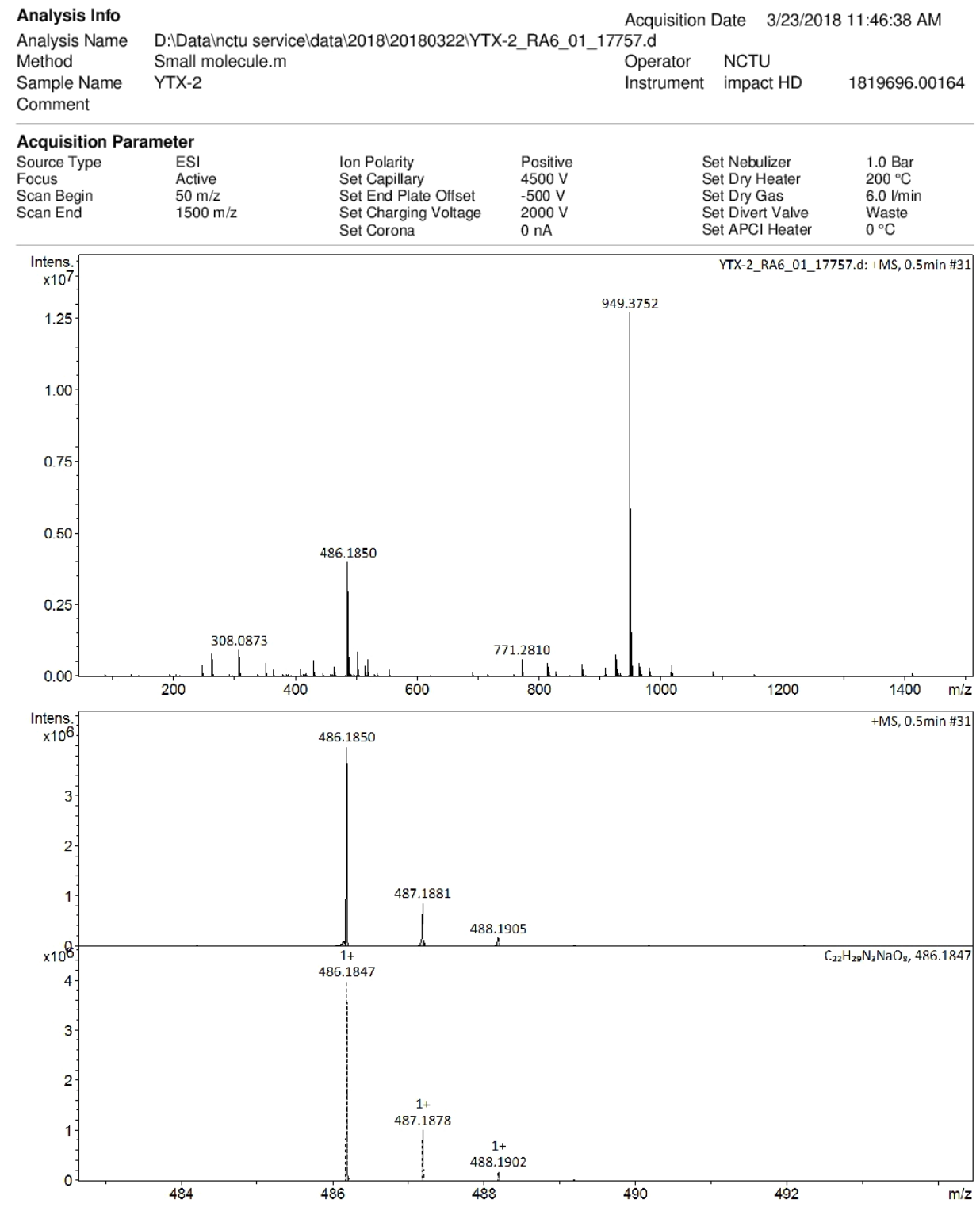

\section{HR ESI-MS}

1,3-(2- $N)$ diBoc-6-nitro tryptophan methyl ester $\boldsymbol{p}$-13 


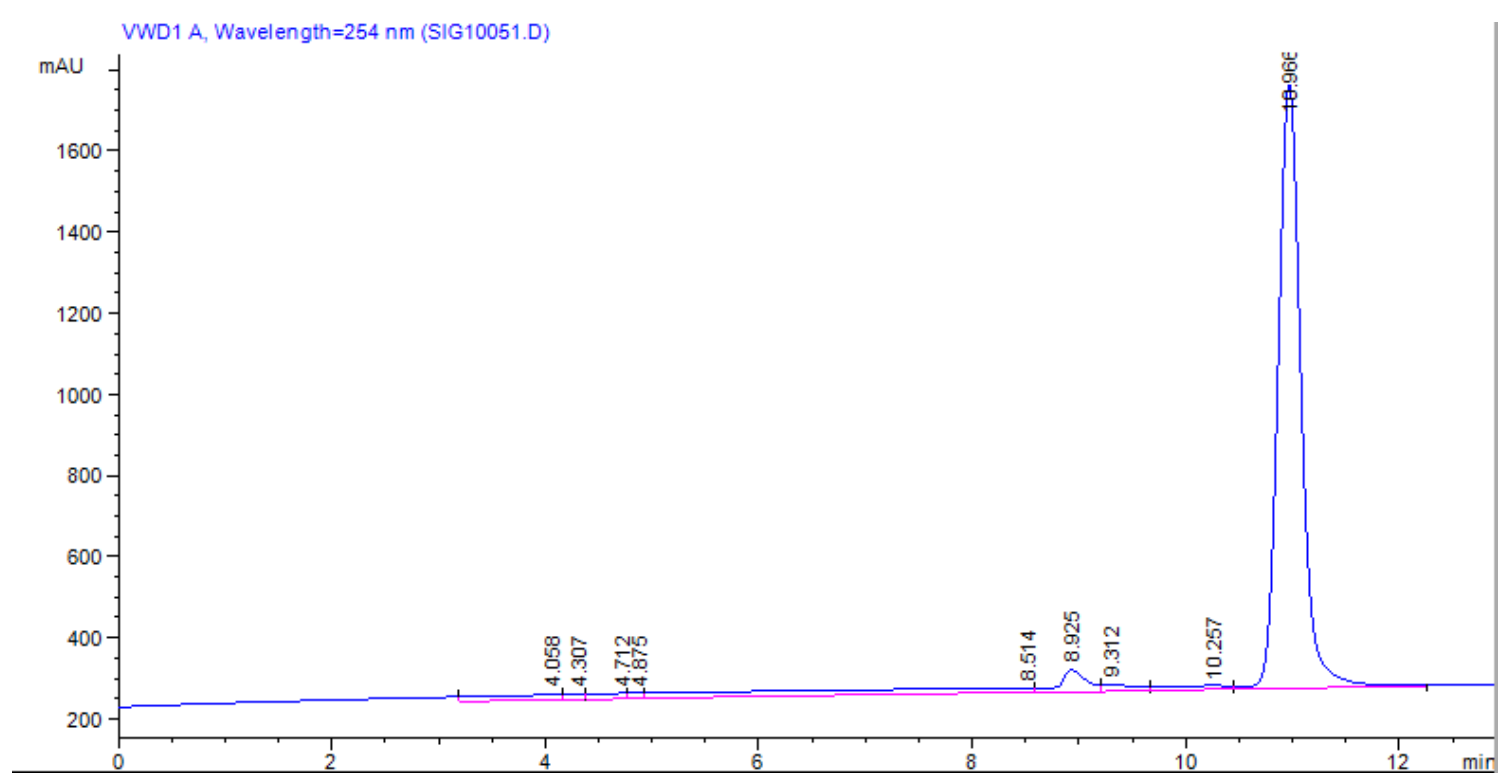

\section{HPLC chromatogram}

1,3-(2-N)diBoc-6-nitro tryptophan methyl ester $\boldsymbol{p}$-13

HPLC column: ZORBAX SIL 5 um $9.4 \times 250$ mm (normal phase), $5 \mu$ L, eluents: EtOAc/n-hexane 2.5:8.5;

flow rate: $3 \mathrm{~mL} / \mathrm{min}, \lambda_{\mathrm{abs}}=254 \mathrm{~nm}$.

Figure 13. Spectra and chromatogram of 1,3-(2-N)diBoc-6-nitro tryptophan methyl ester $\boldsymbol{p}$-13 


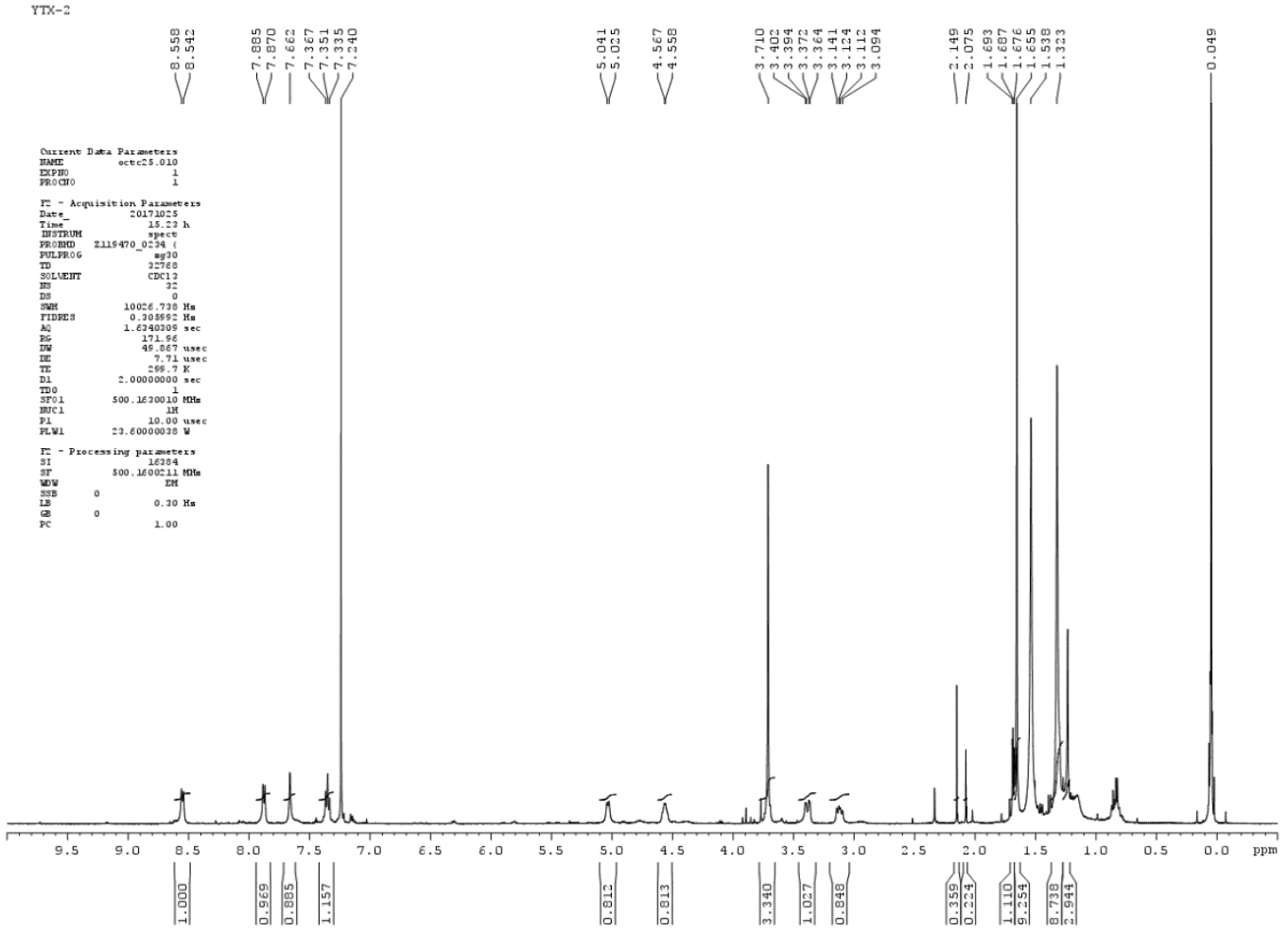

${ }^{1} \mathrm{H}-\mathrm{NMR}$ in $\mathrm{CDCl}_{3}$<smiles>COC(=O)NC(Cc1c[nH]c2cccc([N+](=O)[O-])c12)C(=O)OC</smiles>

1,3-(2-N)diBoc-4-nitro tryptophan methyl ester $\boldsymbol{o}-\mathbf{1 3}$ 


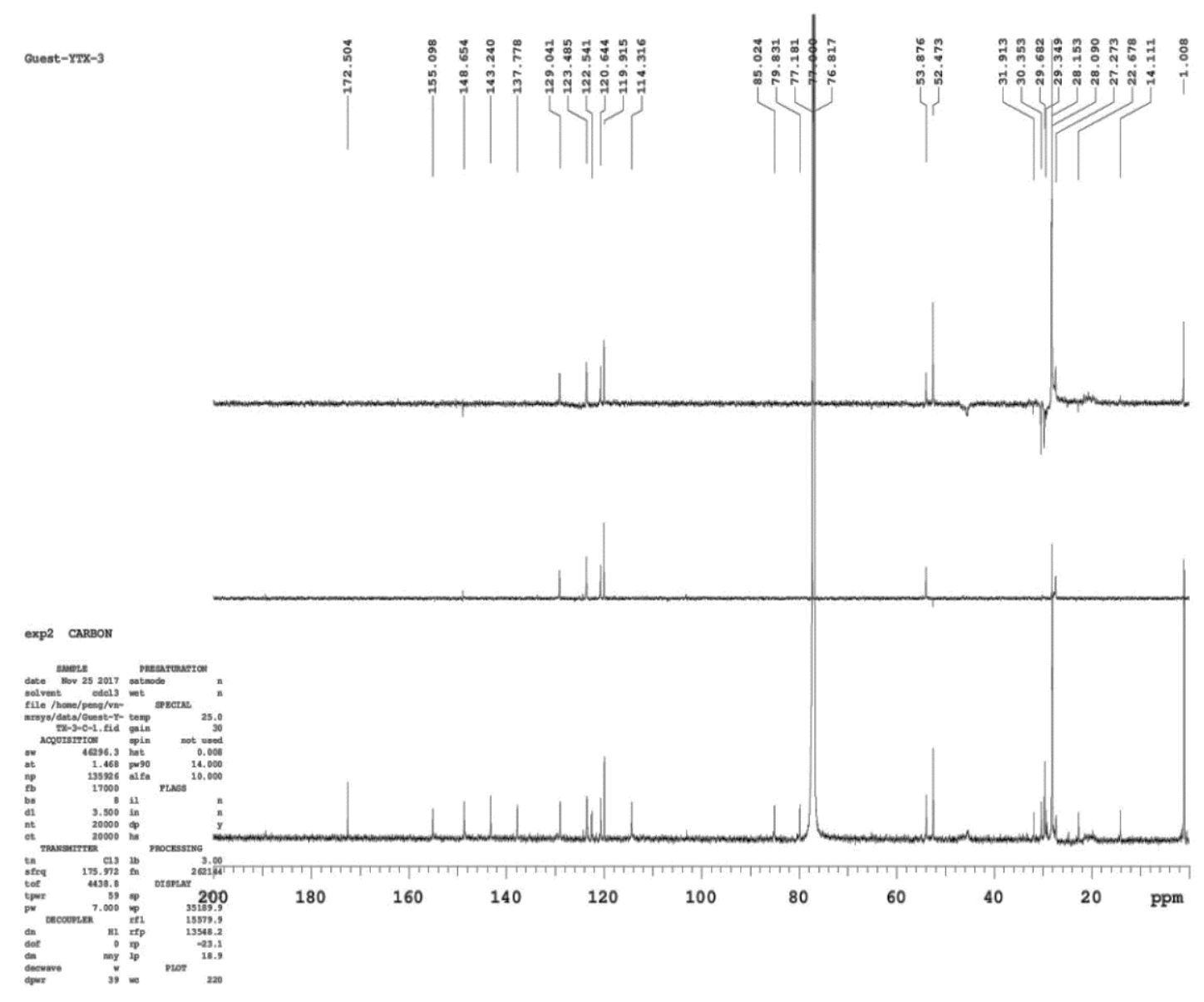

${ }^{13} \mathrm{C}$-DEPT-135 in $\mathrm{CDCl}_{3}$

1,3-(2-N)diBoc-4-nitro tryptophan methyl ester $\boldsymbol{o}-\mathbf{1 3}$ 


\section{Display Report}

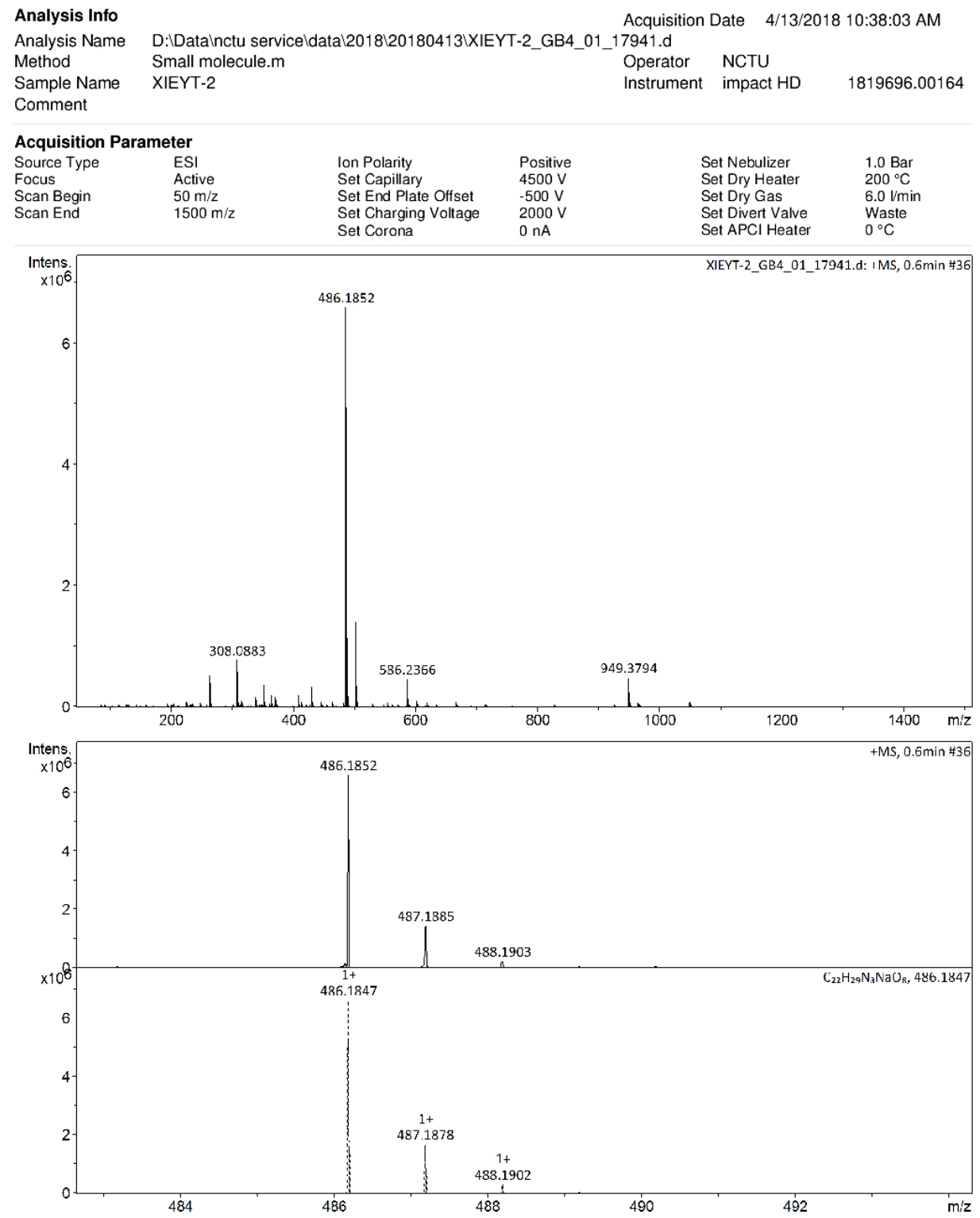

\section{HR ESI-MS}

1,3-(2- $N)$ diBoc-4-nitro tryptophan methyl ester $\boldsymbol{o}-\mathbf{1 3}$ 


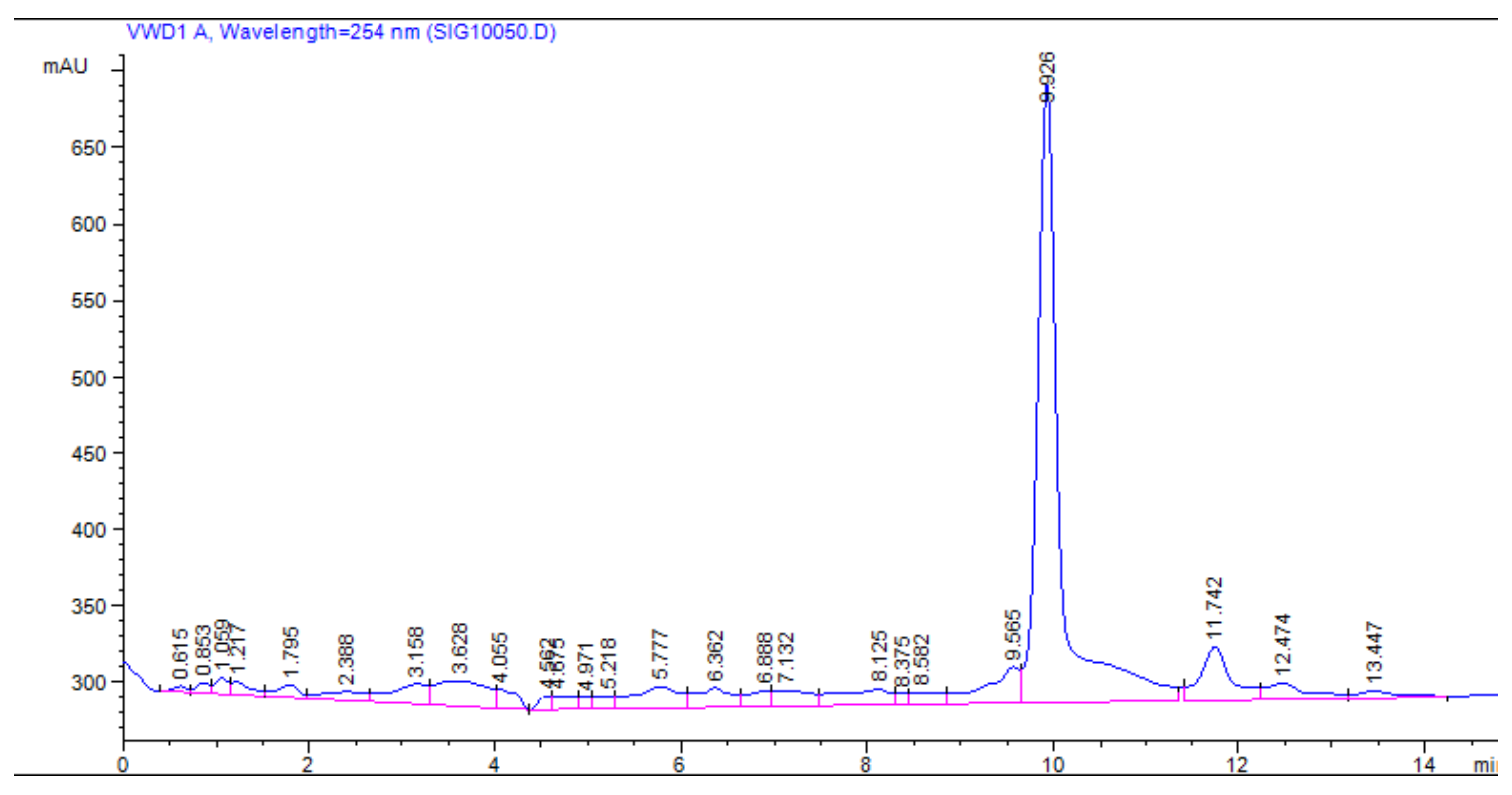

HPLC chromatogram

1,3-(2-N)diBoc-4-nitro tryptophan methyl ester $\boldsymbol{o - 1 3}$

HPLC column: ZORBAX SIL 5 um $9.4 \times 250 \mathrm{~mm}$ (normal phase), injection $5 \mu \mathrm{L}$, eluents: EtOAc/n-hexane 2.5:8.5; flow rate: $3 \mathrm{~mL} / \mathrm{min}, \lambda_{\mathrm{abs}}=254 \mathrm{~nm}$.

Figure 14. Spectra and chromatogram of 1,3-(2-N)diBoc-4-nitro tryptophan methyl ester $\boldsymbol{o}-\mathbf{1 3}$ 


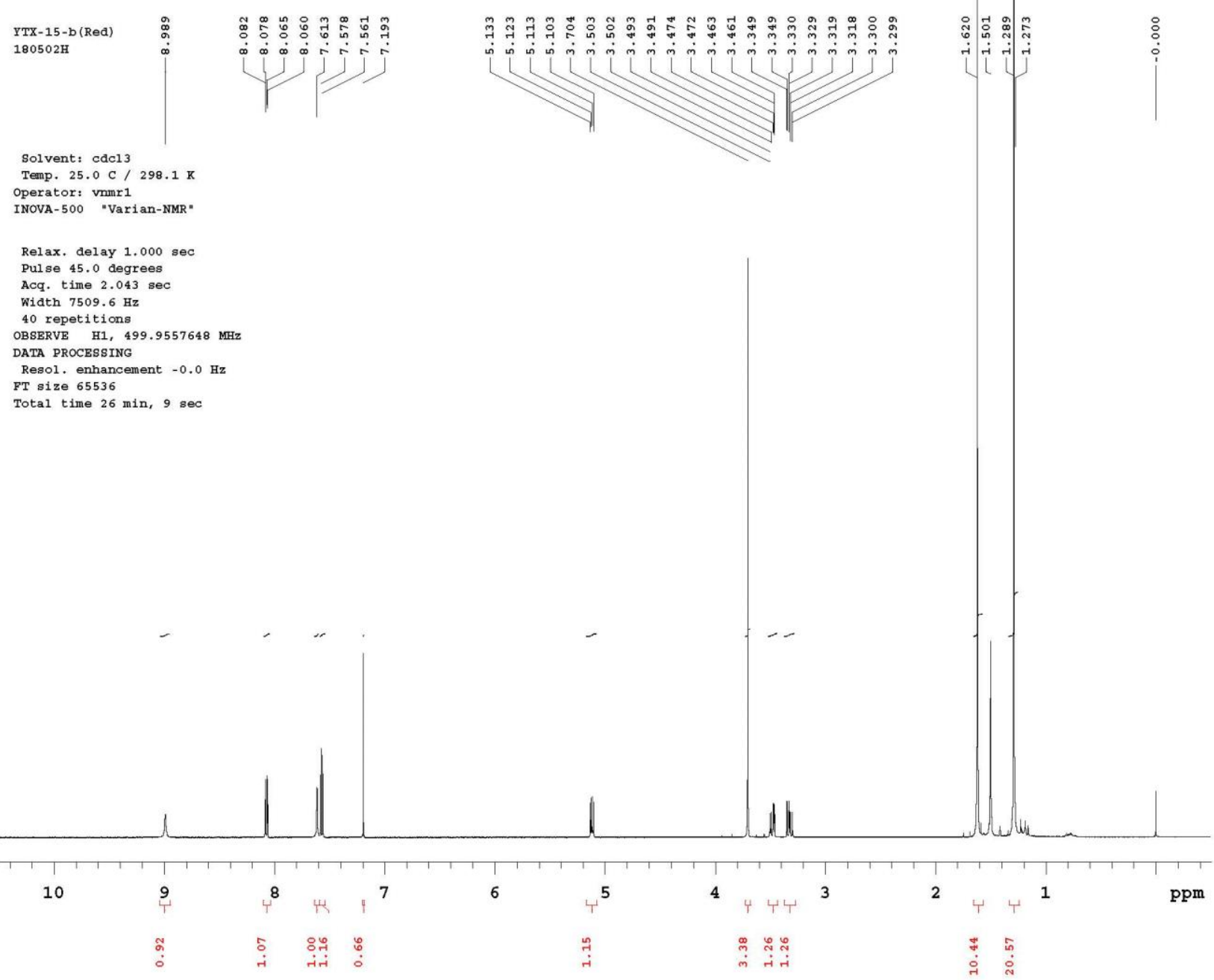

${ }^{1} \mathrm{H}-\mathrm{NMR}$ in $\mathrm{CDCl}_{3}$<smiles>COC(=O)C(Cc1c[nH]c2cc([N+](=O)[O-])ccc12)C(=O)OCc1ccccc1</smiles>

1,3-(2-N,N)triBoc-6-nitro tryptophan methyl ester $\boldsymbol{p}$-14 


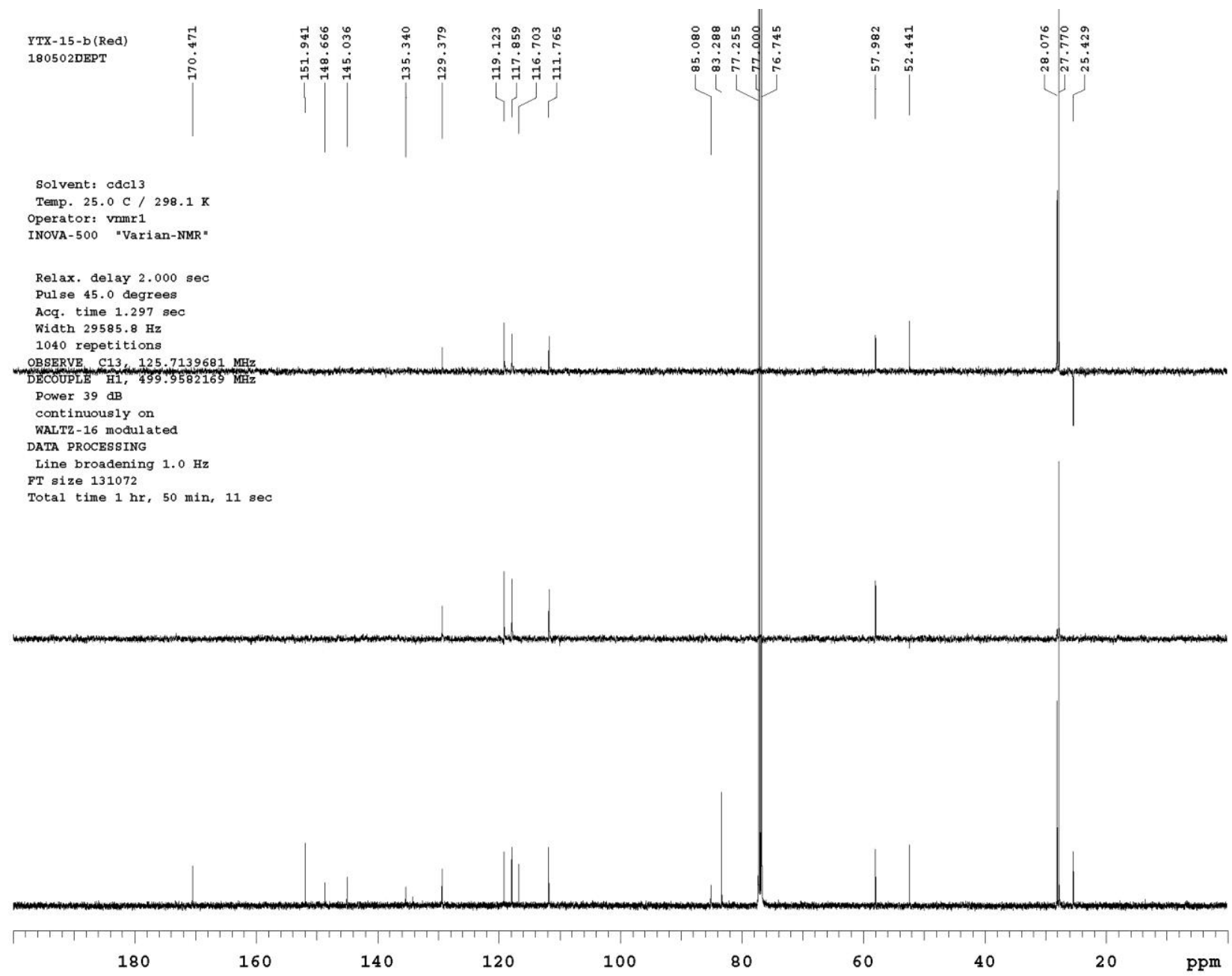

\section{${ }^{13} \mathrm{C}$-DEPT $135 \mathrm{NMR}$ in $\mathrm{CDCl}_{3}$}

1,3-(2-N,N)triBoc-6-nitro tryptophan methyl ester $\boldsymbol{p}$-14 


\section{Display Report}

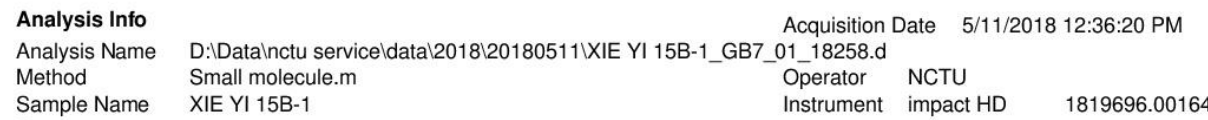
Sample Name
XIE YI 15B-1
Instrument impact $\mathrm{HD}$

\section{Acquisition Parameter}

Source Type ESI

Focus Active

$\begin{array}{ll}\text { Scan Begin } & 50 \mathrm{~m} / \mathrm{z} \\ \text { Scan End } & 1500 \mathrm{~m} / \mathrm{z}\end{array}$

Ion Polarity

Set Nebulizer

Set Dry Heater $\quad 200^{\circ} \mathrm{C}$

$\begin{array}{lll}\text { Set Charging Voltage } & 2000 \mathrm{~V} & \text { Set Divert Valve } \\ \text { Set APCI Heater } & 0^{\circ} \mathrm{C} C\end{array}$

Intens. XIE YI 15B-1_GB7_01_18258.d: IMS, 0.6min \#33
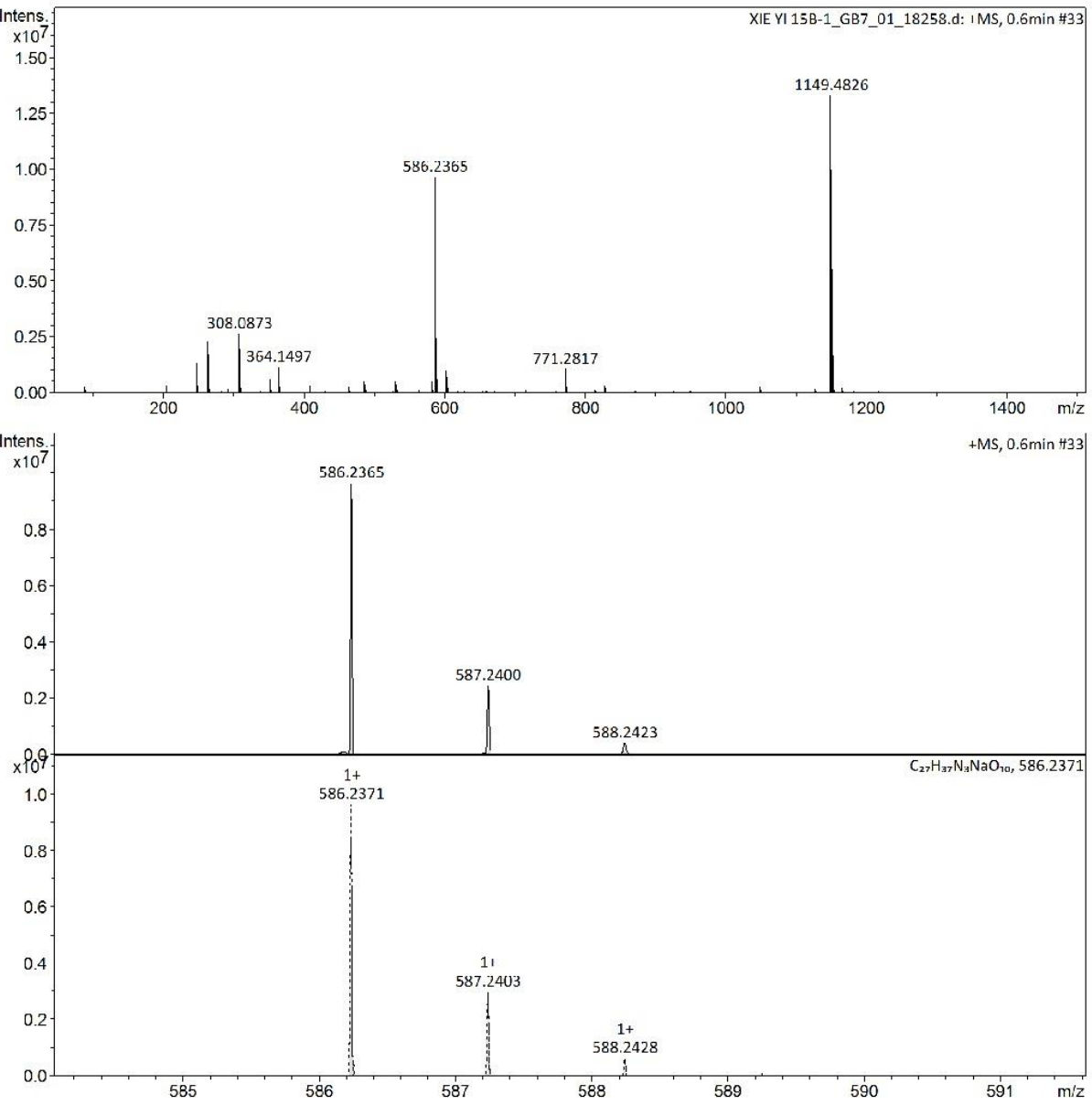

\section{HR ESI-MS}

1,3-(2-N,N)triBoc-6-nitro tryptophan methyl ester $\boldsymbol{p}-\mathbf{1 4}$ 


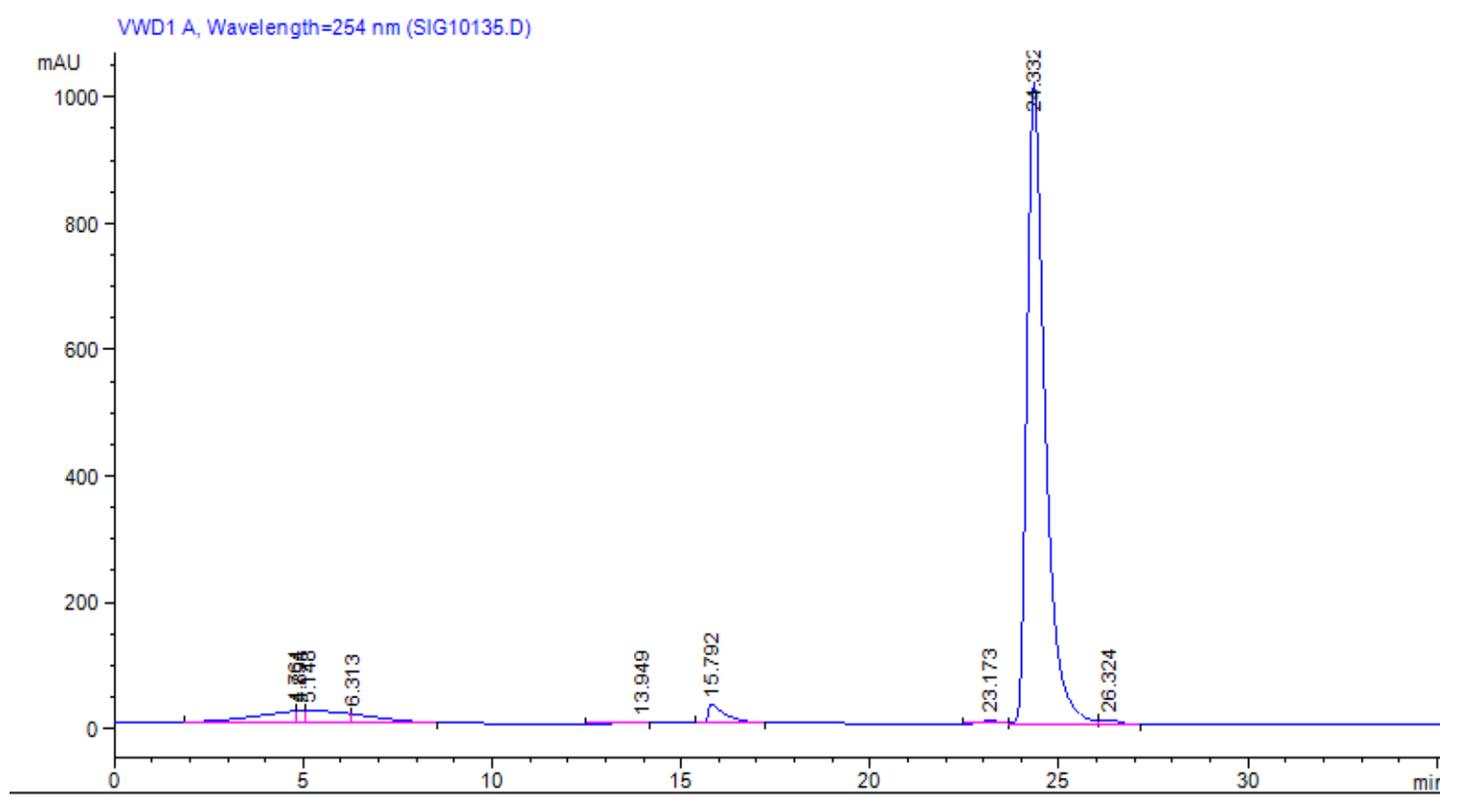

HPLC chromatogram of 1,3-(2-N,N)triBoc-6-nitro tryptophan methyl ester $\boldsymbol{p}$-14

HPLC column: ZORBAX SIL 5 um $9.4 \times 250$ mm (normal phase), injection: $5 \mu \mathrm{L}$, eluents: EtOAc/ $n$ hexane $=1 / 7$; flow rate: $3 \mathrm{~mL} / \mathrm{min}, \lambda_{\mathrm{abs}}=254 \mathrm{~nm}$.

Figure 15. Spectra and chromatogram of 1,3-(2-N,N)triBoc-6-nitro tryptophan methyl ester $\boldsymbol{p}$-14 


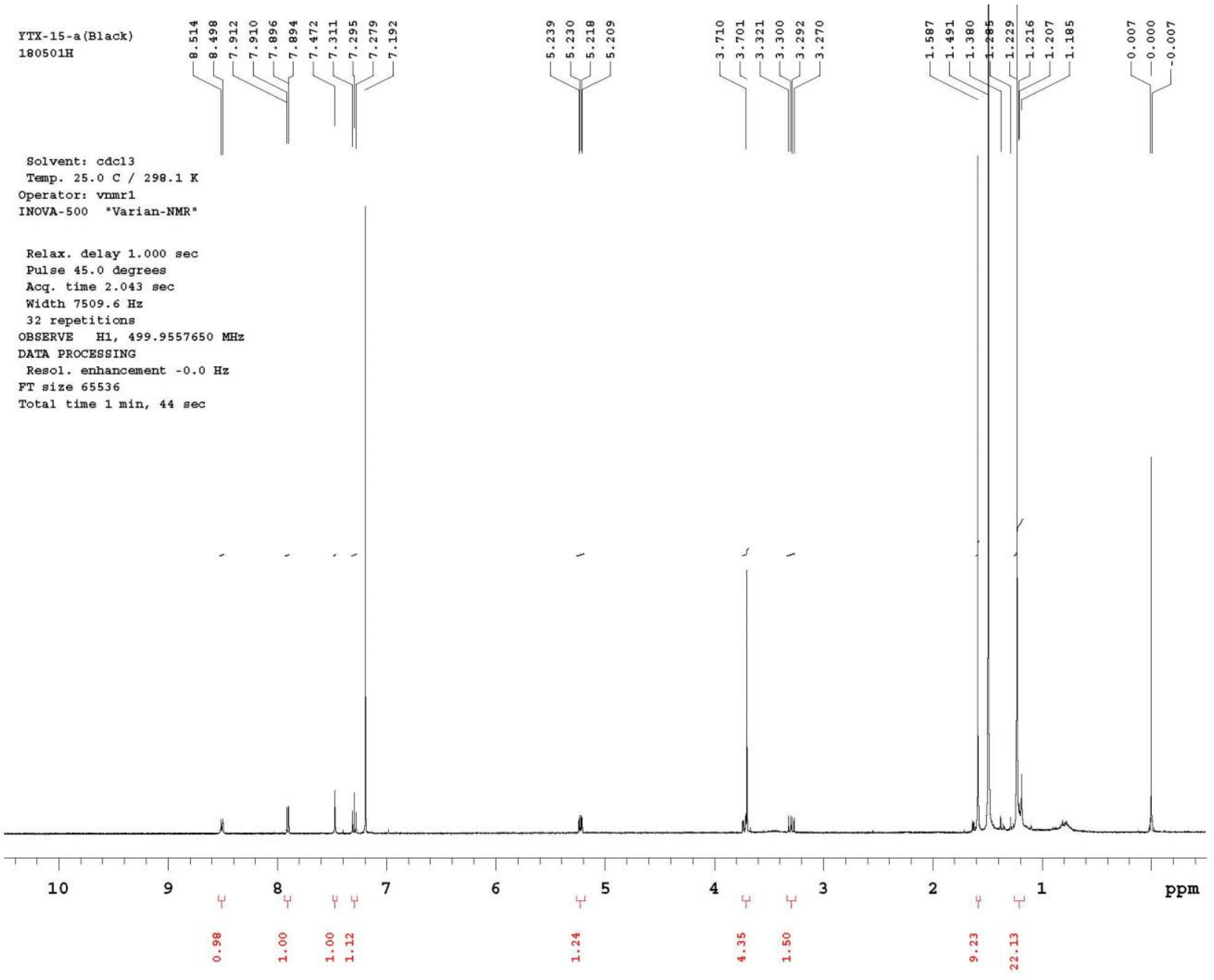

${ }^{1} \mathrm{H}-\mathrm{NMR}$ in $\mathrm{CDCl}_{3}$<smiles>CCOC(=O)OC(Cc1c[nH]c2cccc([N+](=O)[O-])c12)C(=O)OCc1ccccc1</smiles>

1,3-(2-N,N)triBoc-4-nitro tryptophan methyl ester $\boldsymbol{o}-\mathbf{1 4}$ 
YTX-15-a (B1ack)
180501DEPT

Solvent: cdc13

Temp. $25.0 \mathrm{C} / 298.1 \mathrm{~K}$

operator: vnmr

pulse 90.0 degrees

Acq. time $1.001 \mathrm{sec}$

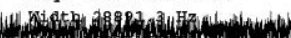

OBSERVE C13, 125.7139716 MH

DECOUPLE H1４99.9582169 MH

Power

on during acquisition

off during delay

DATA PROCESSING

DATA PROCESSING
Line broadening 2.0 He

FT size 65536

Total time $5 \mathrm{hr}, 37 \mathrm{~min}, 22 \mathrm{sec}$

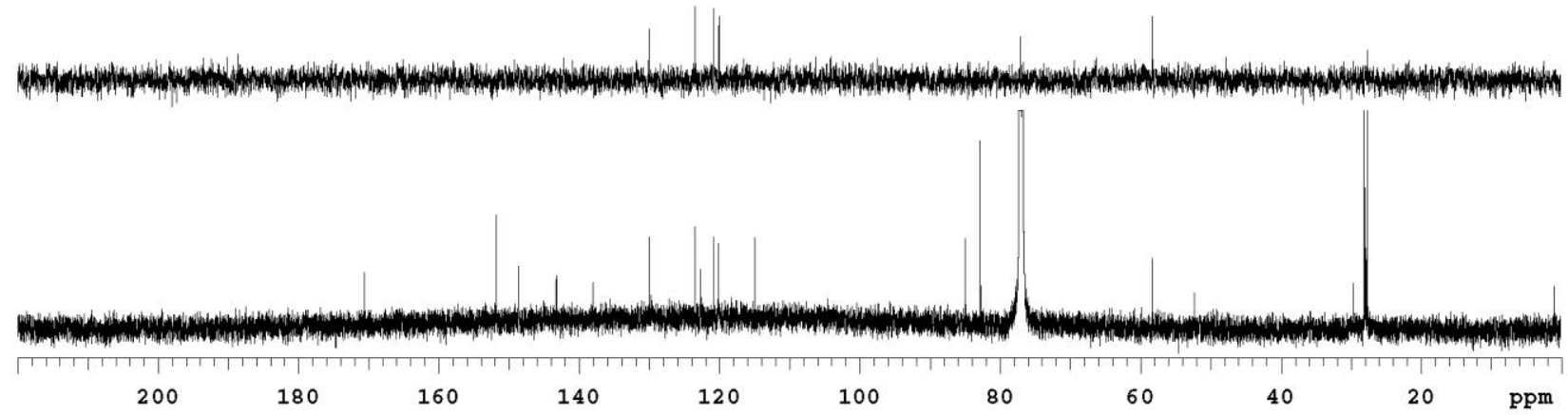

${ }^{13} \mathrm{C}$-DEPT $135 \mathrm{NMR}$ in $\mathrm{CDCl}_{3}$

1,3-(2-N,N)triBoc-6-nitro tryptophan methyl ester $\boldsymbol{o}-14$ 


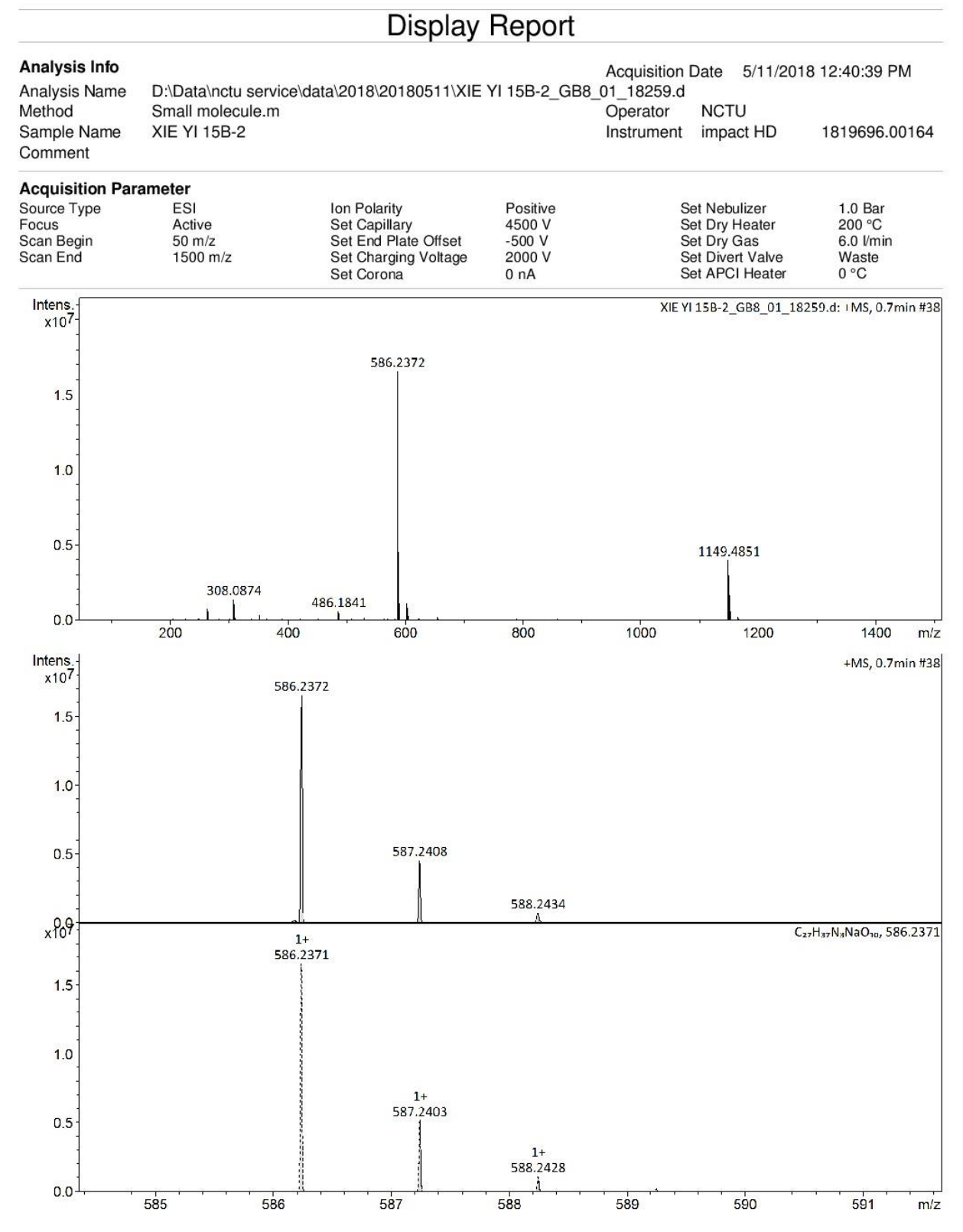

\section{HR ESI-MS}

1,3-(2- $N, N)$ triBoc-4-nitro tryptophan methyl ester $\boldsymbol{o}-14$ 


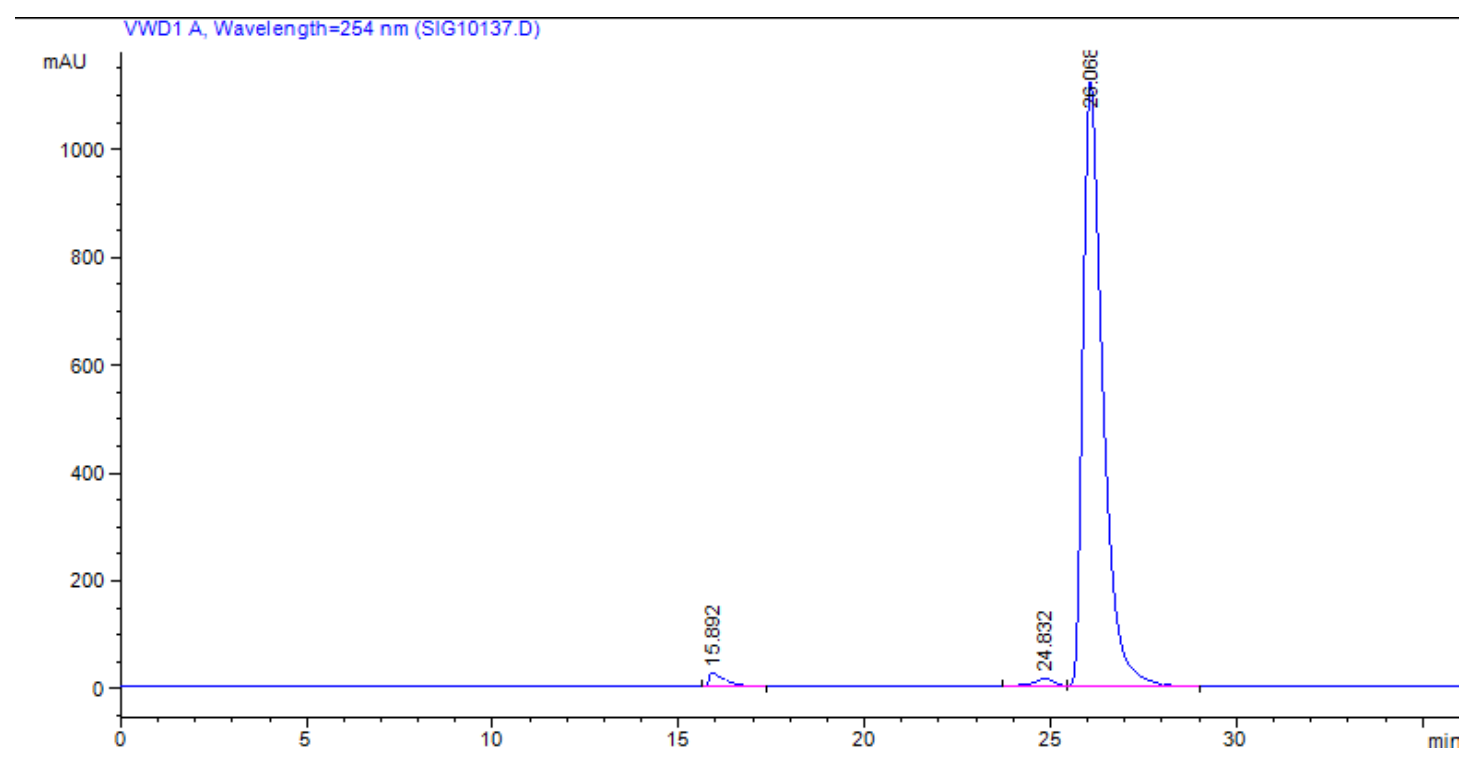

HPLC chromatogram

1,3-(2-N,N)triBoc-4-nitro tryptophan methyl ester $\boldsymbol{o}-\mathbf{1 4}$

HPLC column: ZORBAX SIL 5 um 9.4 x 250 mm (normal phase), injection: $5 \mu \mathrm{L}$, eluents: EtOAc/n-hexane $=1 / 7$; flow rate: $3 \mathrm{~mL} / \mathrm{min}, \lambda_{\mathrm{abs}}=254 \mathrm{~nm}$.

Figure 16. Spectra and chromatogram of 1,3-(2- $N, N)$ triBoc-4-nitro tryptophan methyl ester o-14 

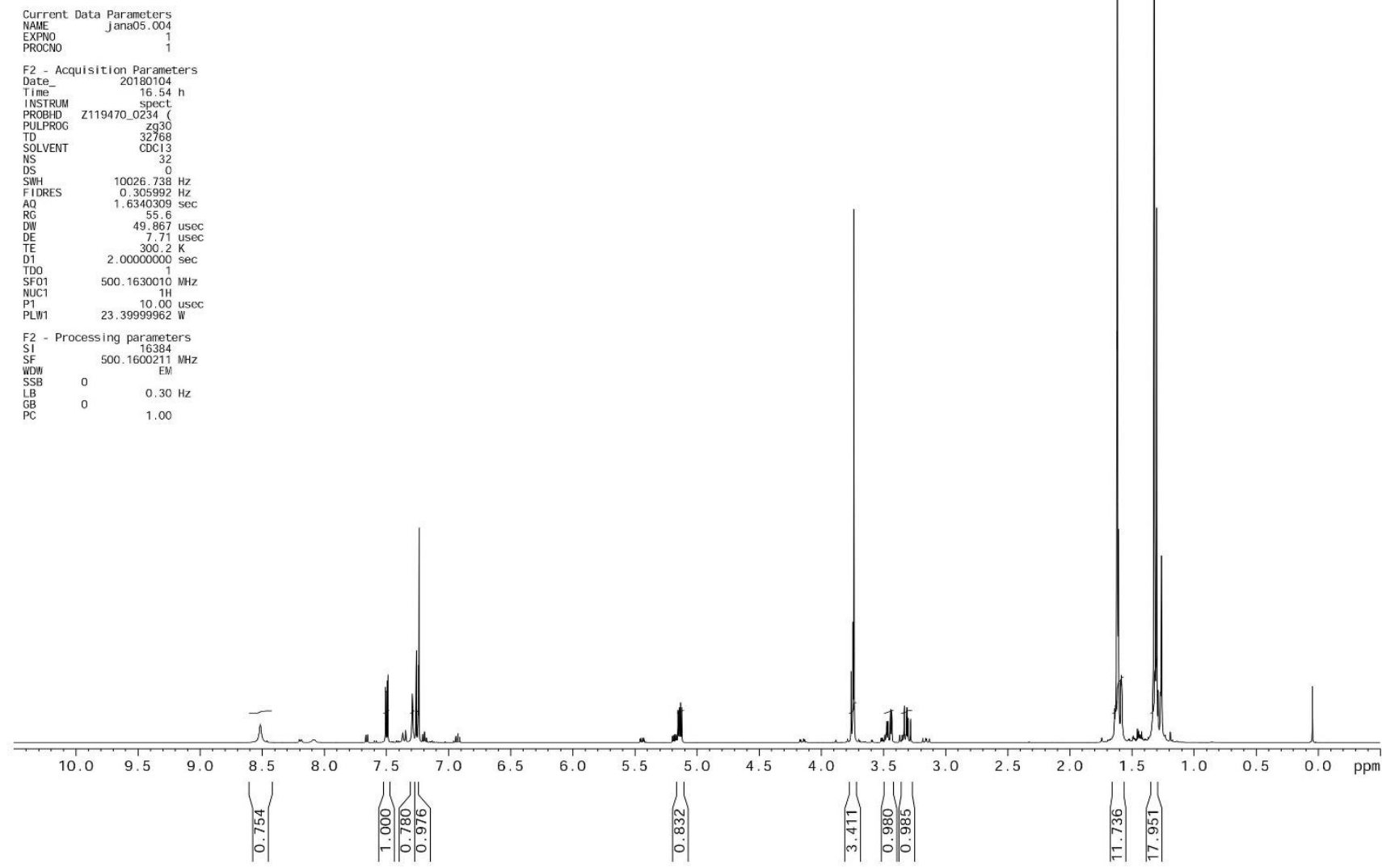

\section{${ }^{1} \mathrm{H}-\mathrm{NMR}$ in $\mathrm{CDCl}_{3}$}<smiles>CC(=O)OC(Cc1c[nH]c2cc(I)ccc12)C(=O)O</smiles><smiles>COC(=O)C(Cc1c[nH]c2cccc(I)c12)C(=O)OC</smiles><smiles>CC(=O)OCC(Cc1c[nH]c2ccccc12)C(=O)O</smiles><smiles>CC(=O)OC(C)(C)C</smiles>

Boc

1,3-(2-N,N)triBoc-6-iodo tryptophan methyl ester $\boldsymbol{p}-\mathbf{1 6}, 1,3-(2-N, N)$ triBoc-4-iodo tryptophan methyl ester $\boldsymbol{o}$ 16 and deiodinated derivative 


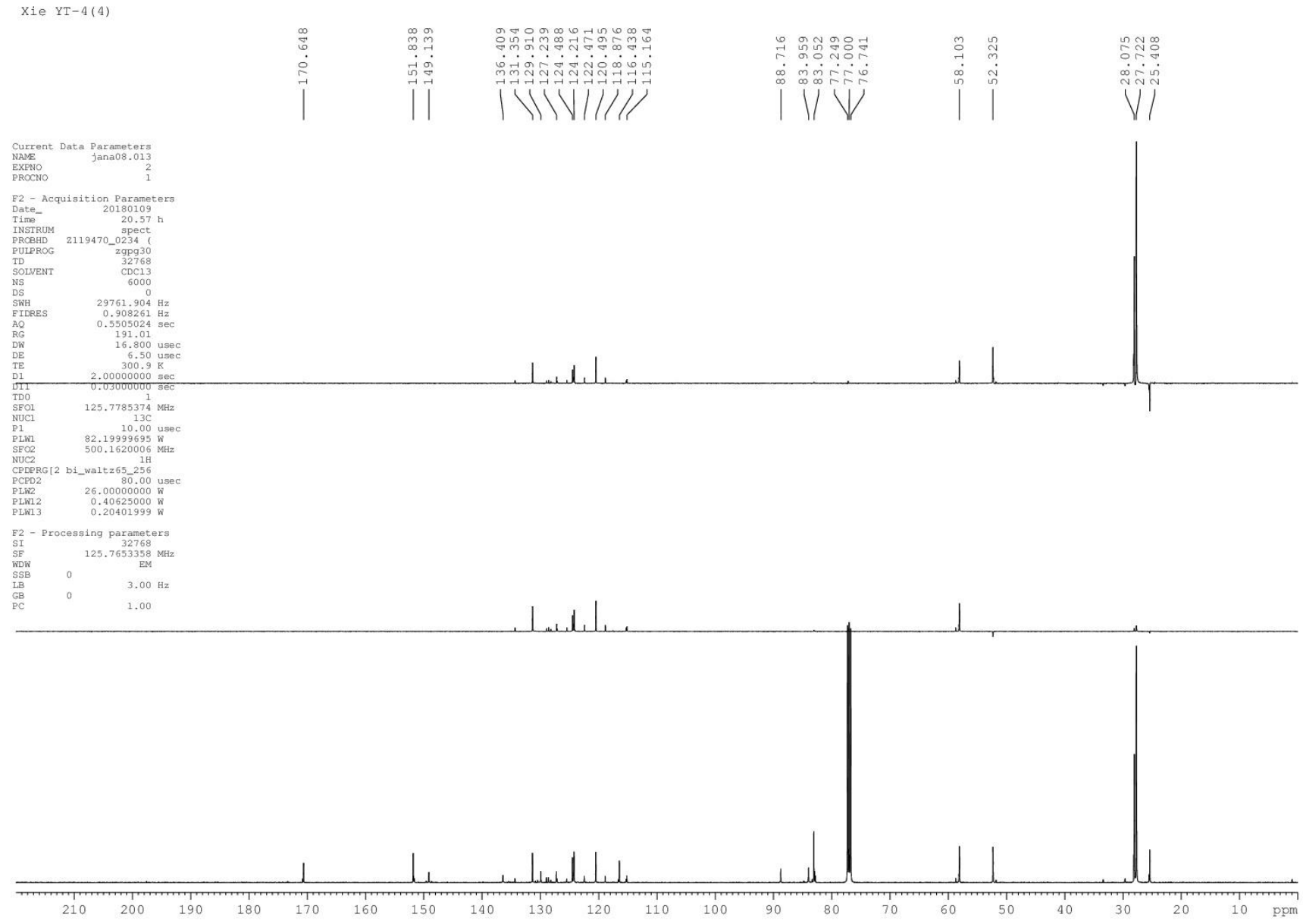

${ }^{13} \mathrm{C}$-DEPT $135 \mathrm{NMR}$ in $\mathrm{CDCl}_{3}$

1,3-(2-N,N)triBoc-6-iodo tryptophan methyl ester $\boldsymbol{p}-\mathbf{1 6}, 1,3-(2-N, N)$ triBoc-4-iodo tryptophan methyl ester $\boldsymbol{o}$ 16 and deiodinated derivative 


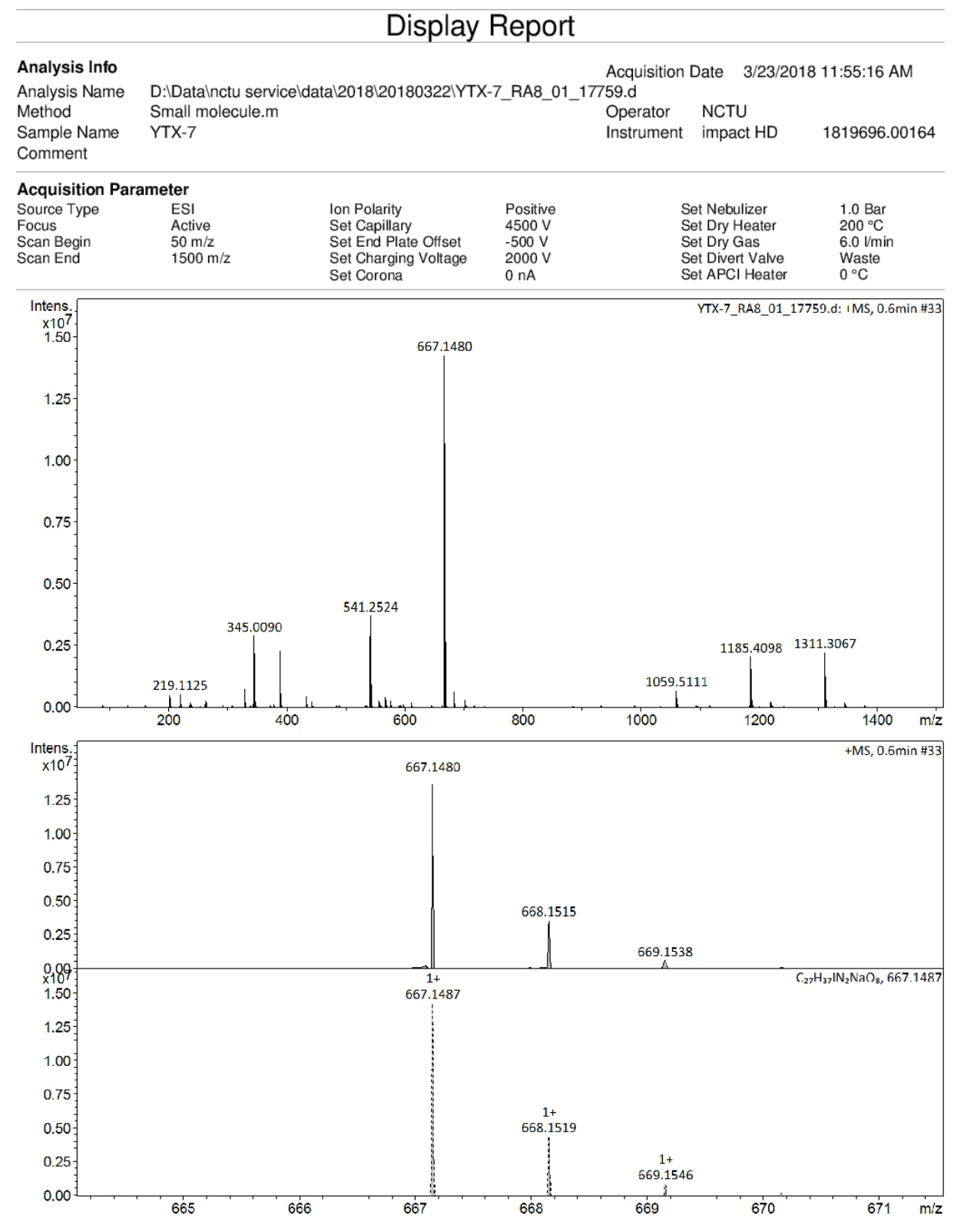

\section{HR ESI-MS}

1,3-(2-N,N)triBoc-6-iodo tryptophan methyl ester $\boldsymbol{p}-\mathbf{1 6}, 1,3-(2-N, N)$ triBoc-4-iodo tryptophan methyl ester $\boldsymbol{o}$ -

16 and deiodinated derivative 


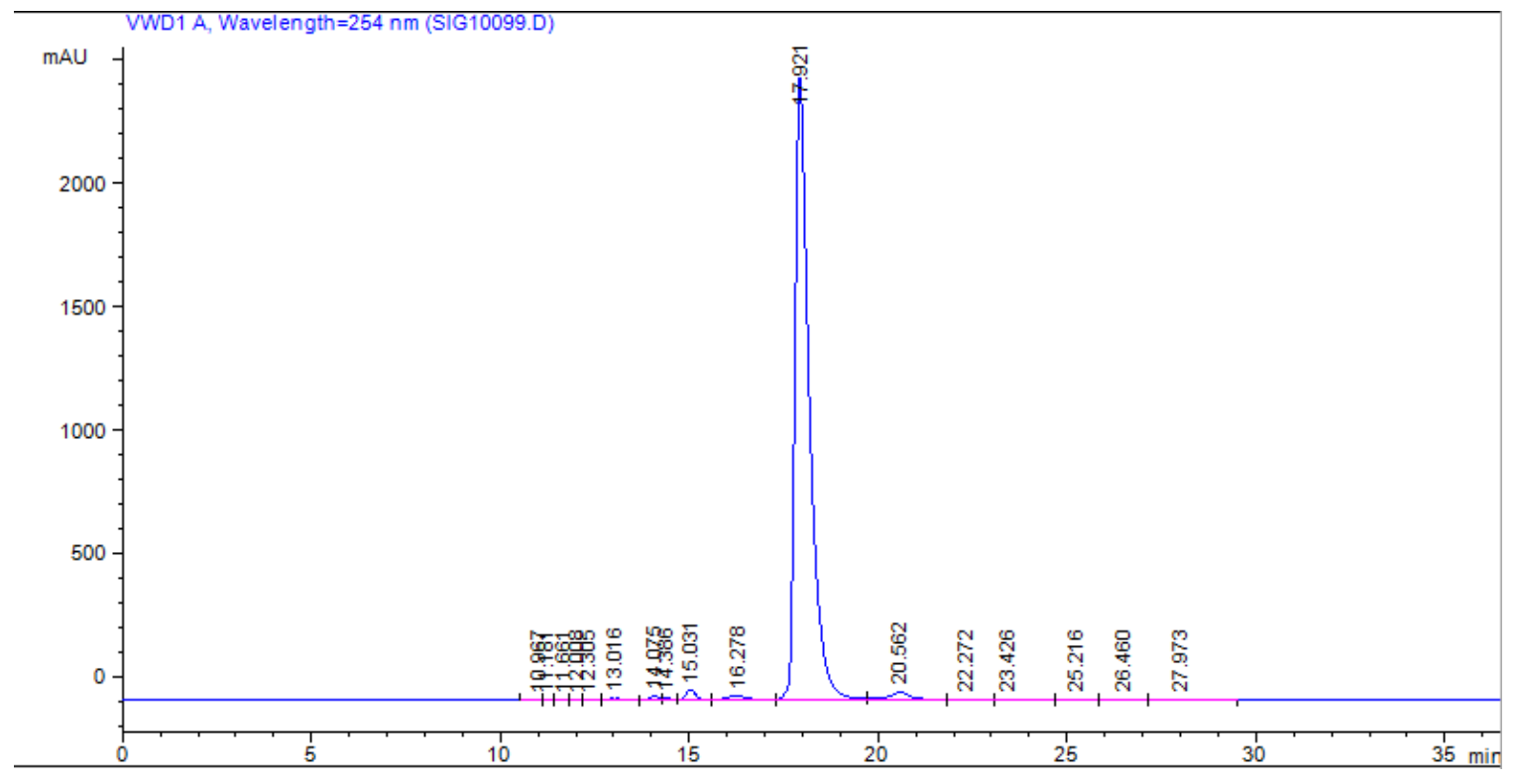

HPLC chromatogram

1,3-(2-N,N)triBoc-6-iodo tryptophan methyl ester $\boldsymbol{p}-\mathbf{1 6}, 1,3-(2-N, N)$ triBoc-4-iodo tryptophan methyl ester $\boldsymbol{o}$ 16 and deiodinated derivative A mixture of $\boldsymbol{p}-\mathbf{1 6}, \boldsymbol{o - 1 6}(19 \mathrm{mg} / 0.6 \mathrm{~mL})$ and deiodinated derivative was injected in portion of $5 \mathrm{uL}$ using eluents of EtOAc/ $n$-hexane 1:8 and a flow rate of $3 \mathrm{~mL} / \mathrm{min}$. HPLC column: ZORBAX SIL 5 um $9.4 \times 250$ $\mathrm{mm}(\mathrm{Si}-90)$ at $\lambda$ of $254 \mathrm{~nm}$.

Figure 17. Spectra and chromatogram of iodotryptophan derivatives $\boldsymbol{p}-\mathbf{1 6}$ and $\boldsymbol{o - 1 6}$ and the deiodo byproduct 


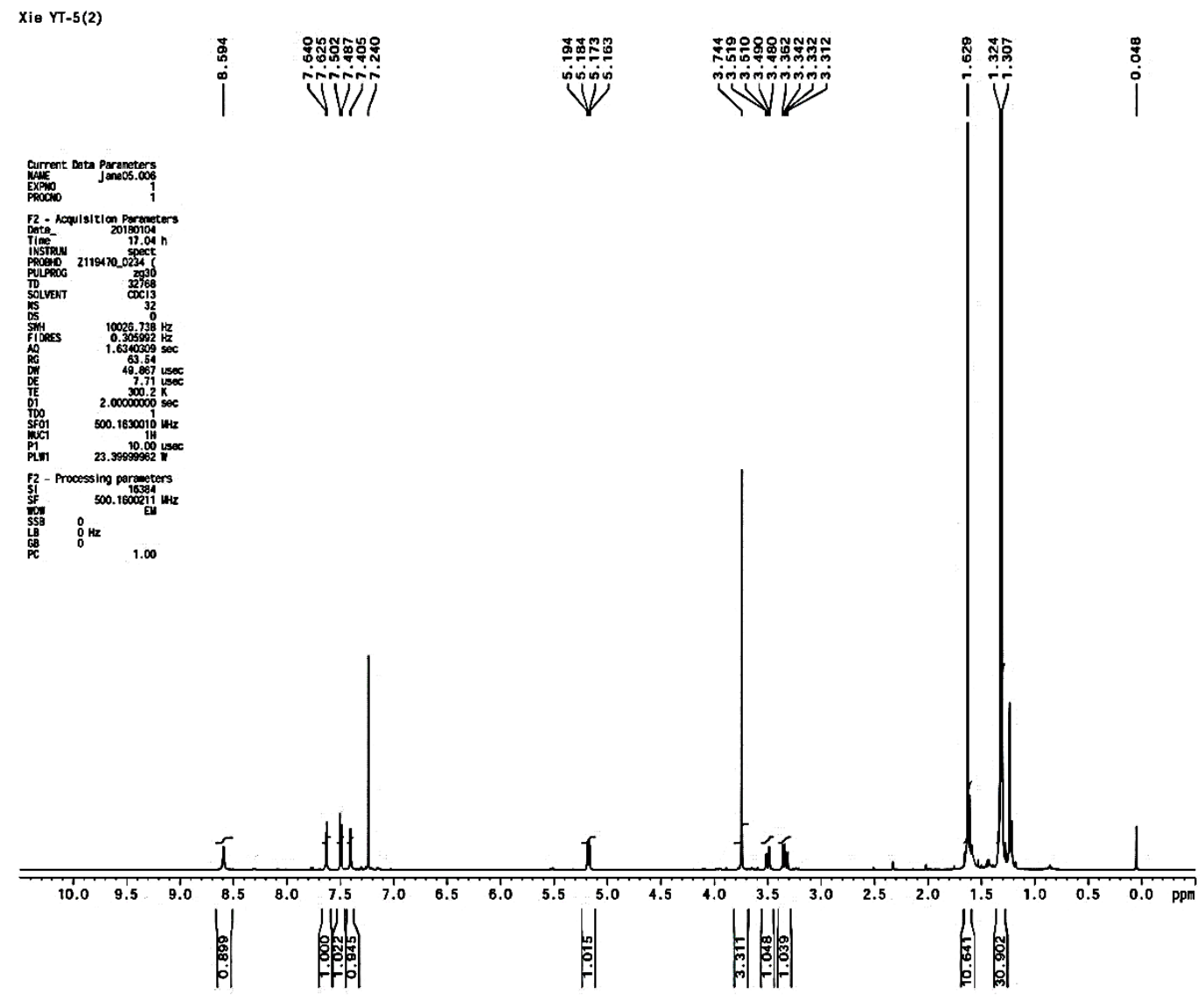

${ }^{1} \mathrm{H}-\mathrm{NMR}$ in $\mathrm{CDCl}_{3}$

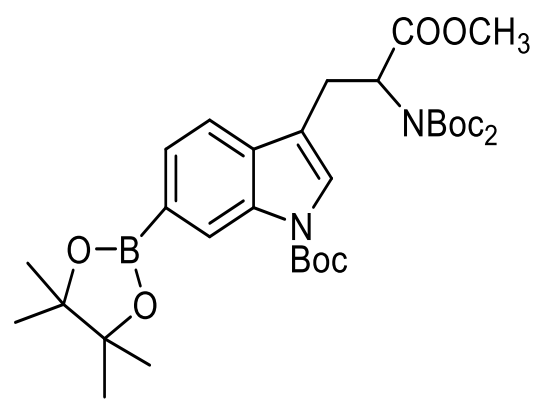

TB-6-BT 5 


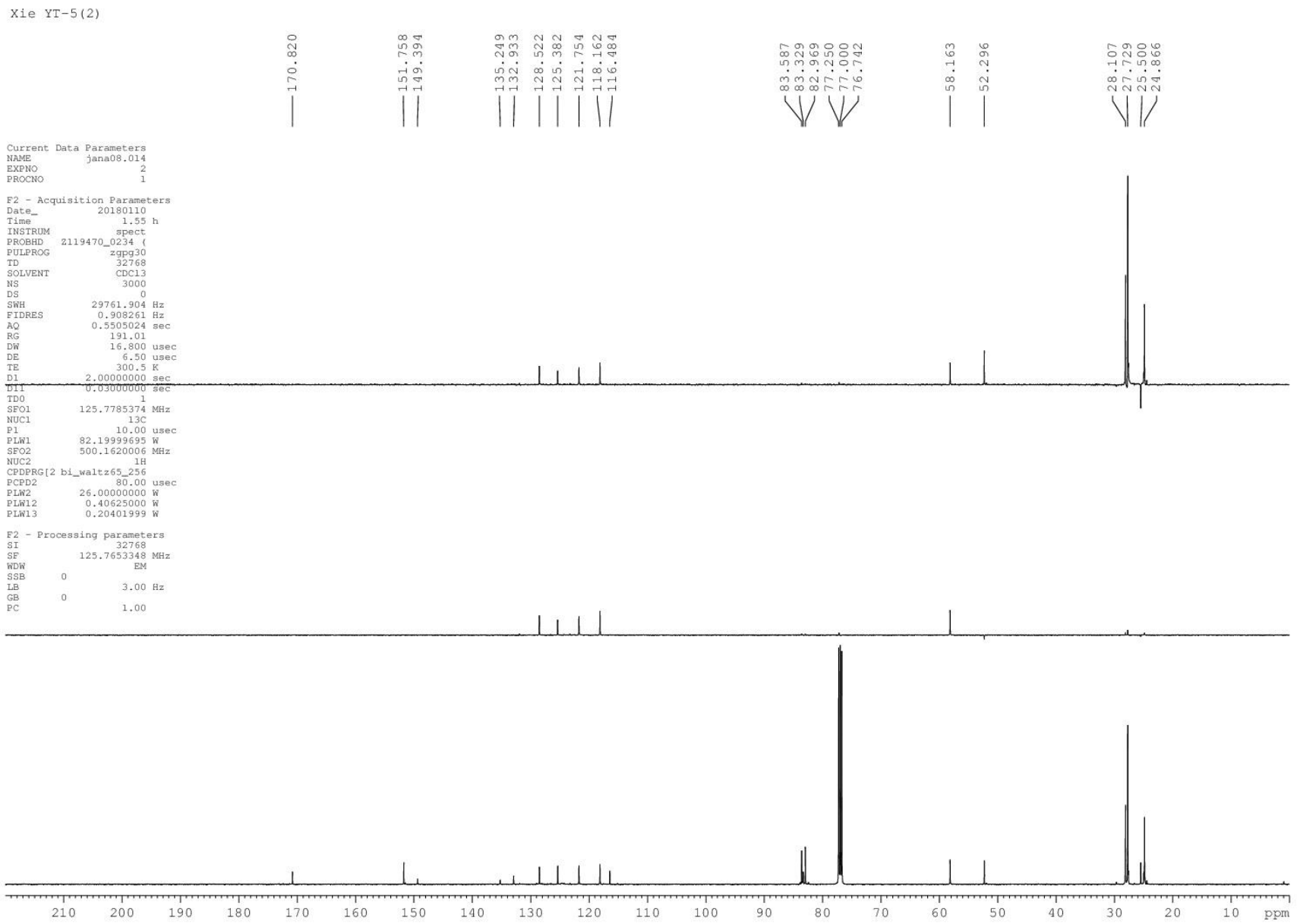

${ }^{13} \mathrm{C}$-DEPT $135 \mathrm{NMR}$ in $\mathrm{CDCl}_{3}$

TB-6BT 5 


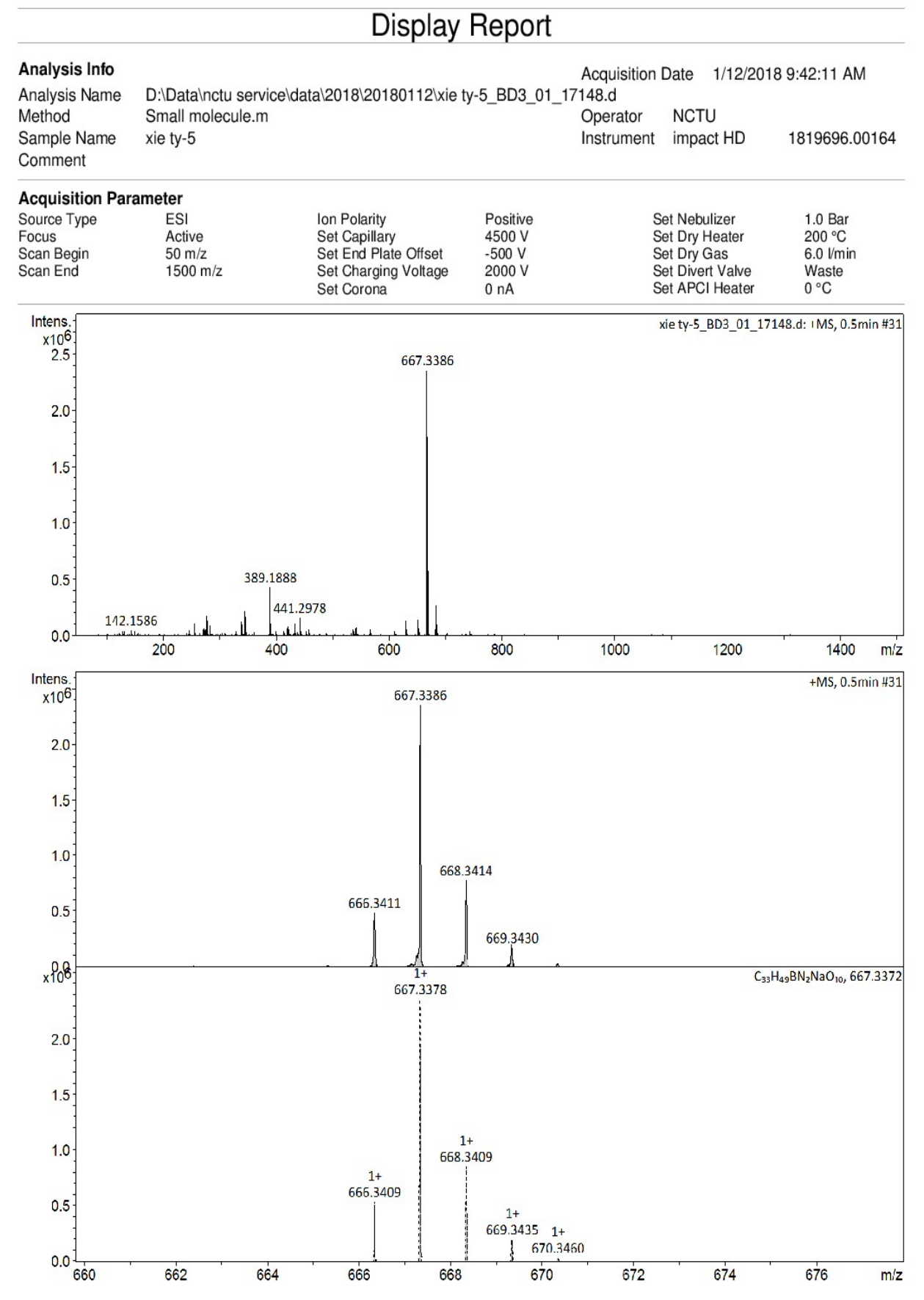

HR ESI - MS

TB-6BT 5

Figure 18. Spectra of 1,3-(2-N,N)triBoc-6-boronopinacol tryptophan methyl ester (TB-6-BT) 5 


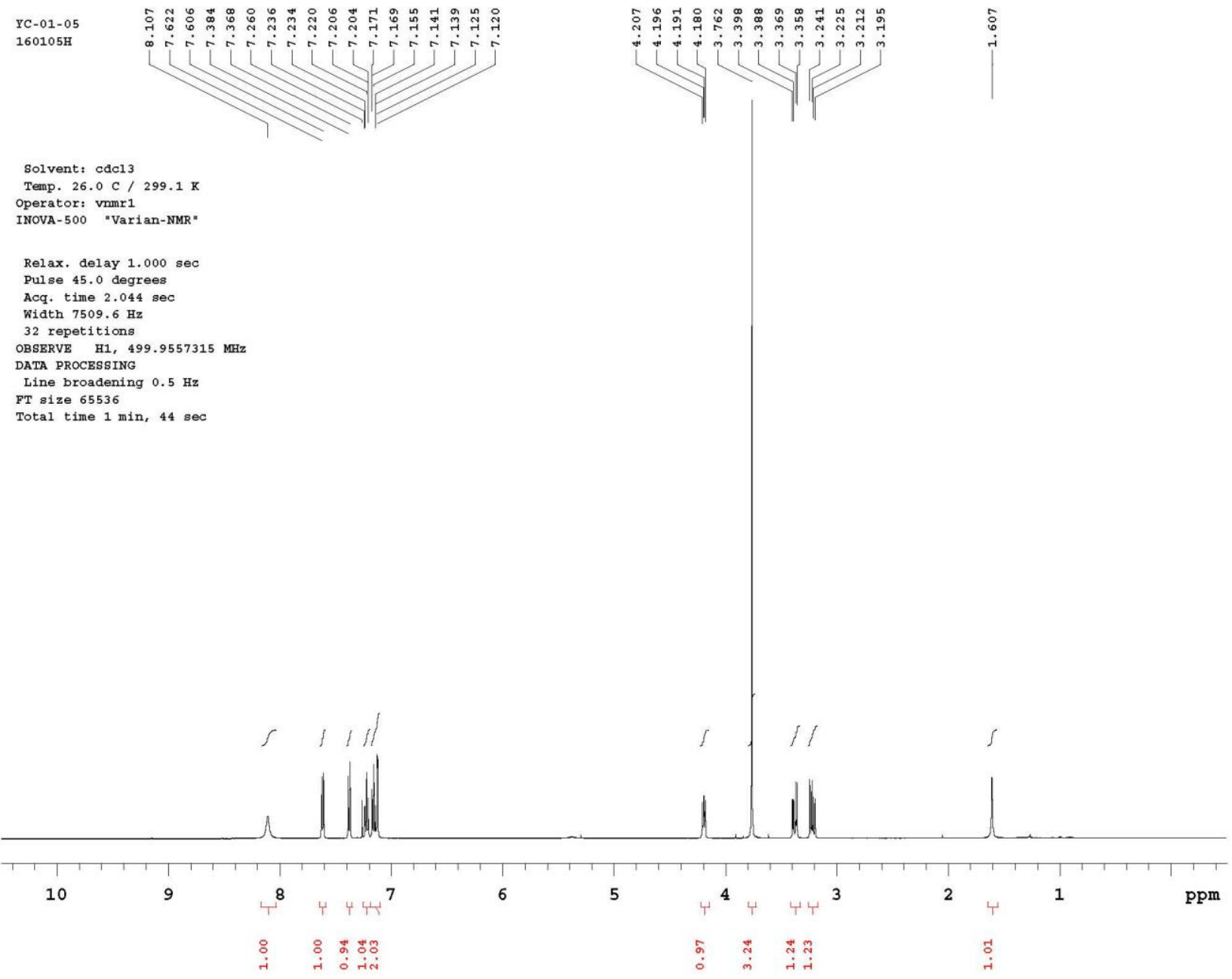

${ }^{1} \mathrm{H}-\mathrm{NMR}$ in $\mathrm{CDCl}_{3}$<smiles>COC(=O)[C@H](N)Cc1c[nH]c2ccccc12</smiles>

3-(2-azidodeamino)tryptophan methyl ester 17 

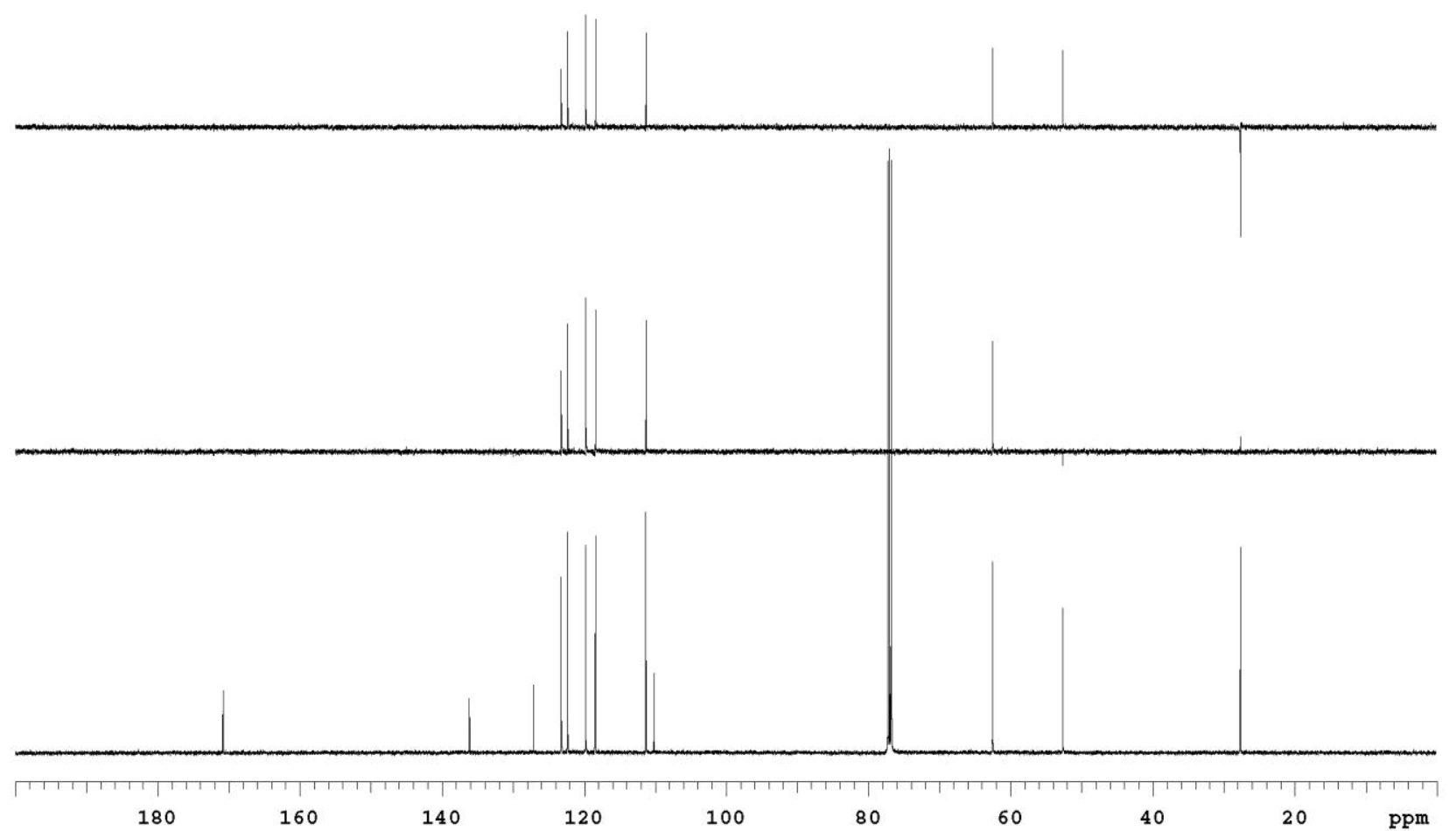

\section{${ }^{13} \mathrm{C}$-DEPT $135 \mathrm{NMR}$ in $\mathrm{CDCl}_{3}$}

3-(2-azidodeamino)tryptophan methyl ester $\mathbf{1 7}$ 


\section{Display Report}
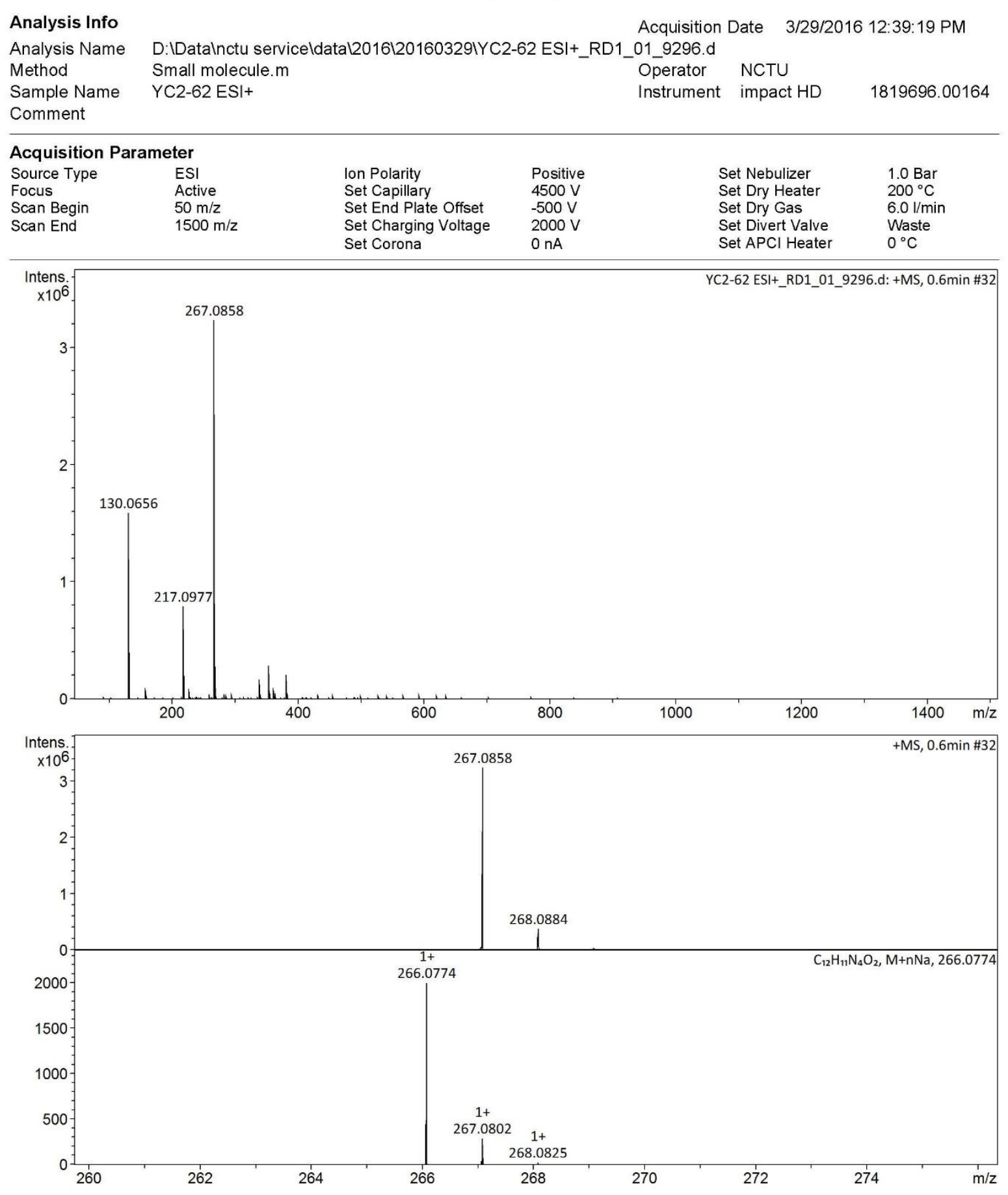

YC2-62 ESI+_RD1_01_9296.d

Bruker Compass DataAnalysis 4.1

\section{HR ESI-MS}

3-(2-azidodeamino)tryptophan methyl ester $\mathbf{1 7}$

Figure 19. Spectra of 3-(2-azidodeamino)tryptophan methyl ester 17 

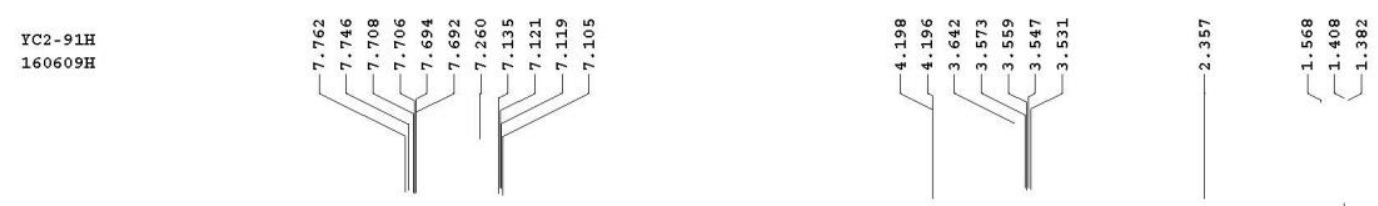

Solvent: cde13

Ambient temperature

operator: vamr 1

File: $160609 \mathrm{H}$

INOVA-500 "Varian-NMR

Relax. delay $1.000 \mathrm{sec}$

Pulse 45.0 degrees

Acq. time $2.046 \mathrm{sec}$

Wiath $7509.6 \mathrm{~Hz}$

32 repetitions

OBSERVE H1, 499.9557318 MHz

DATA PROCESSING

Resol. enhancement $-0.0 \mathrm{~Hz}$

FT size 65536

Total time $1 \mathrm{~min}, 44 \mathrm{sec}$

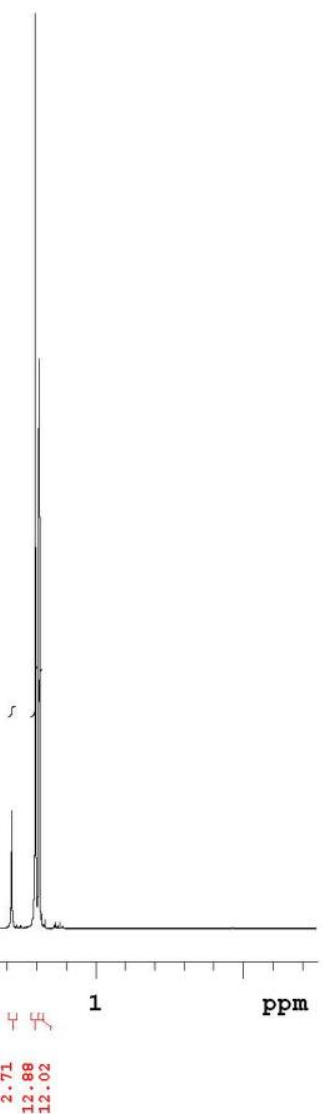

${ }^{1} \mathrm{H}-\mathrm{NMR}$ in $\mathrm{CDCl}_{3}$

AZ-2,7-DBT 6

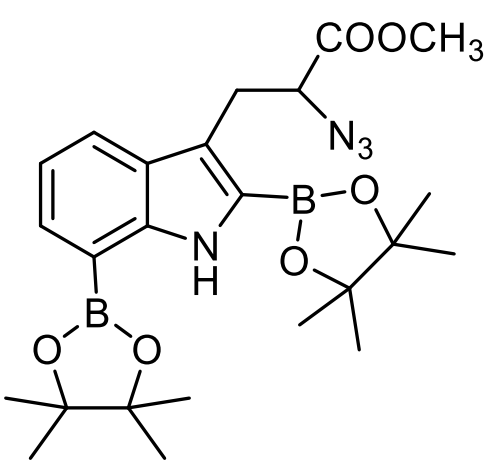




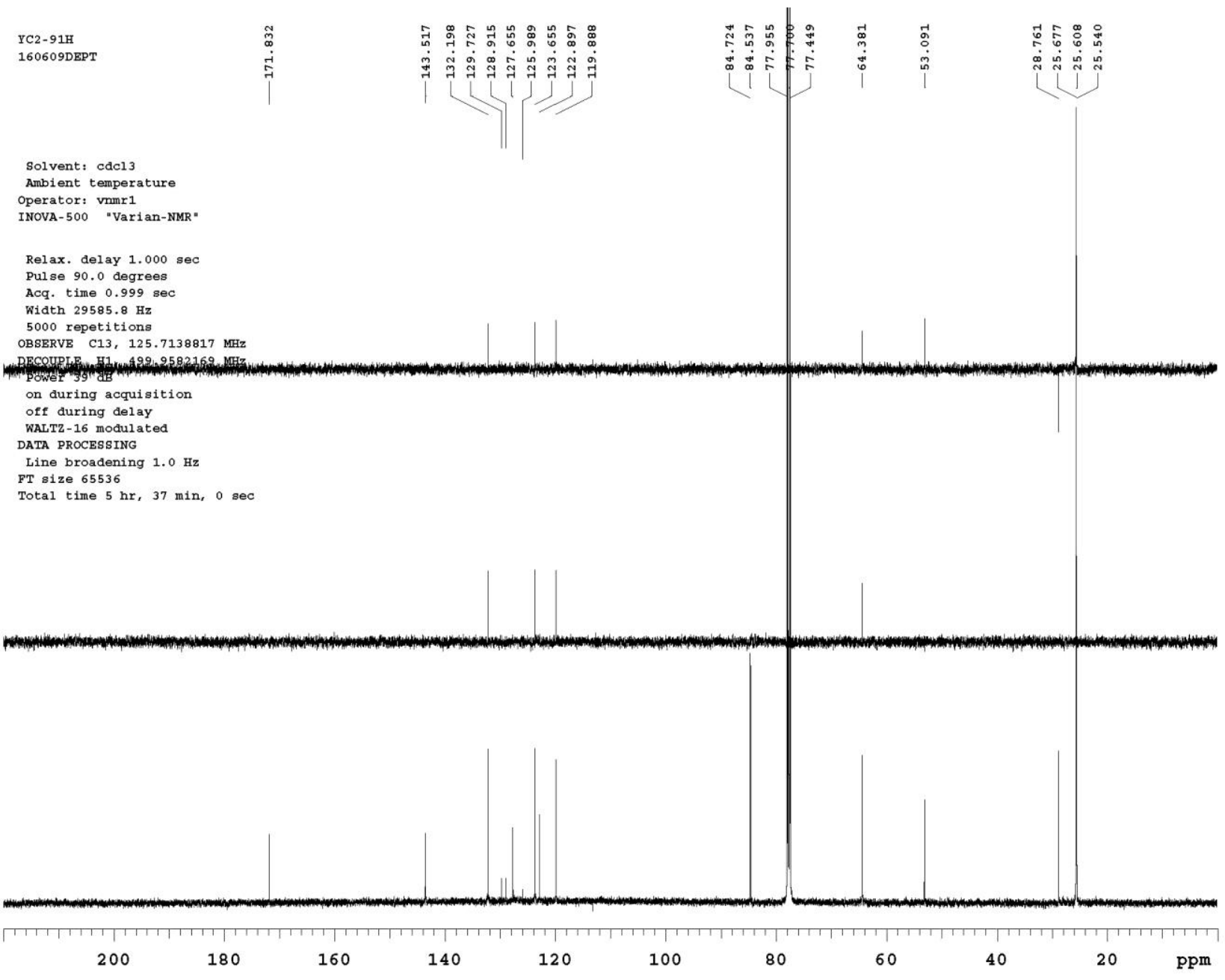

${ }^{13} \mathrm{C}$-DEPT $135 \mathrm{NMR}$ in $\mathrm{CDCl}_{3}$

AZ-2,7-DBT 6 


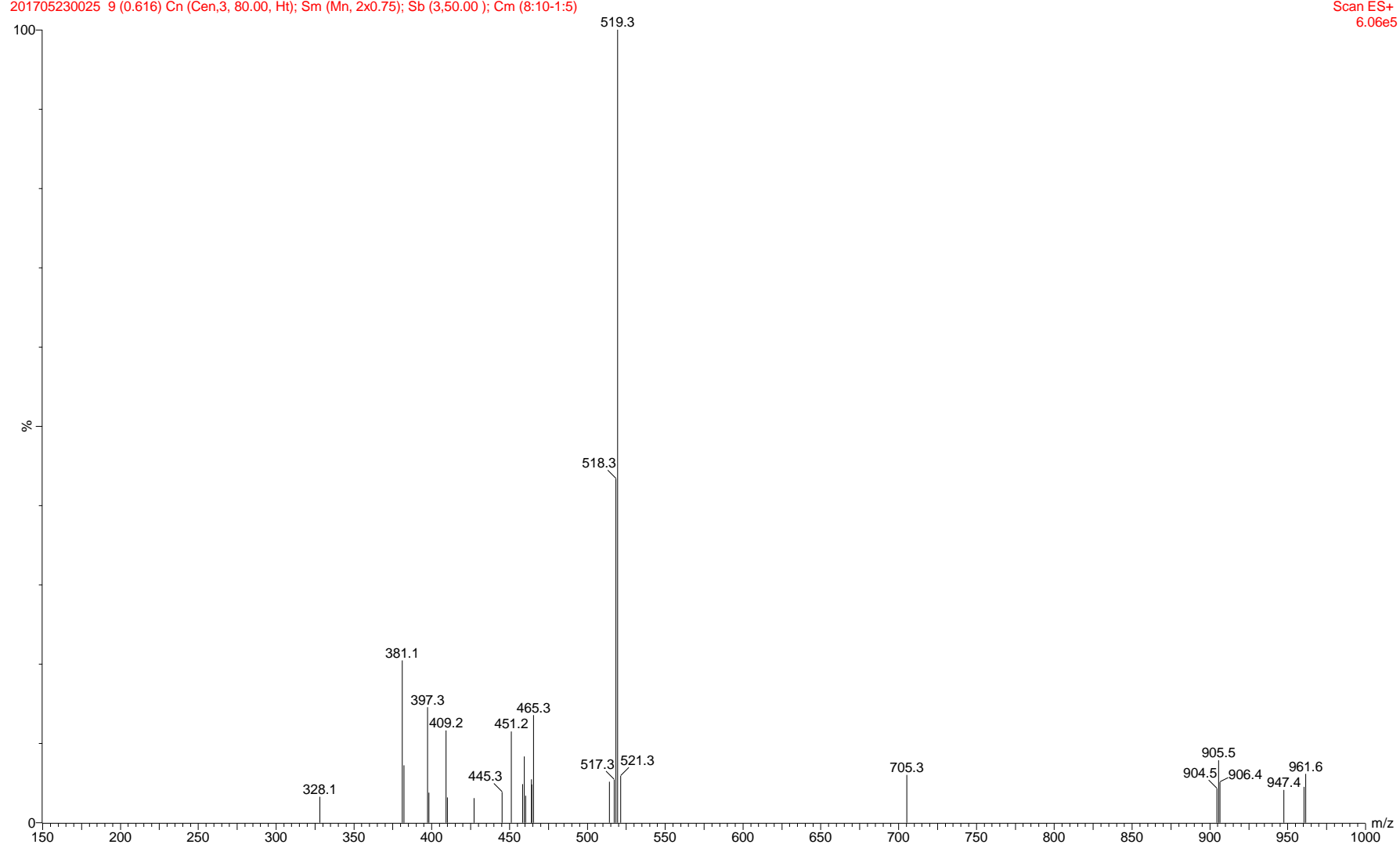

\section{LR ESI-MS}

AZ-2,7-DBT 6 


\section{Display Report}

Analysis Info

Analysis Name

Method

Sample Name

Comment

\section{Acquisition Parameter}

Source Type

Focus

Scan Begin

Scan End

ESI

Active

$1500 \mathrm{~m} / \mathrm{z}$

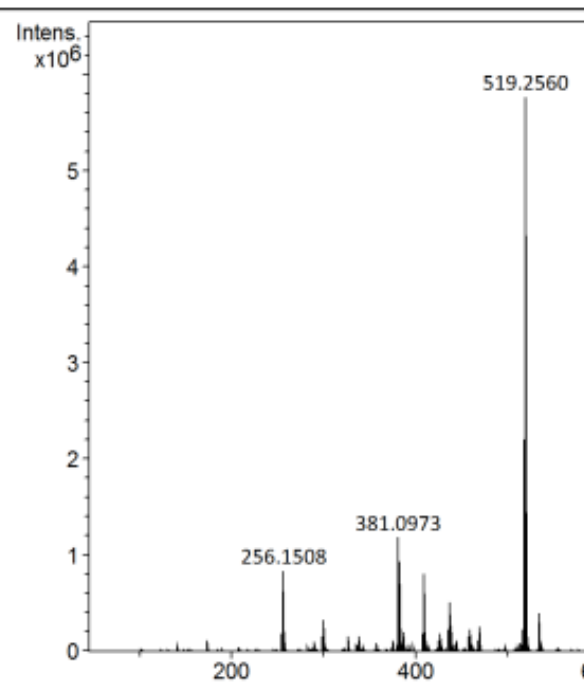

Ion Polarity

$4500 \mathrm{~V}$

Set Find Plate Offset

Set Charging Voltage

$-500 \mathrm{~V}$

$2000 \mathrm{~V}$

$0 \cap A$

Acquisition Date $\quad 5 / 23 / 2017$ 2:54:52 PM

625

Operator NCTU

Instrument impact HD $\quad 1819696.00164$
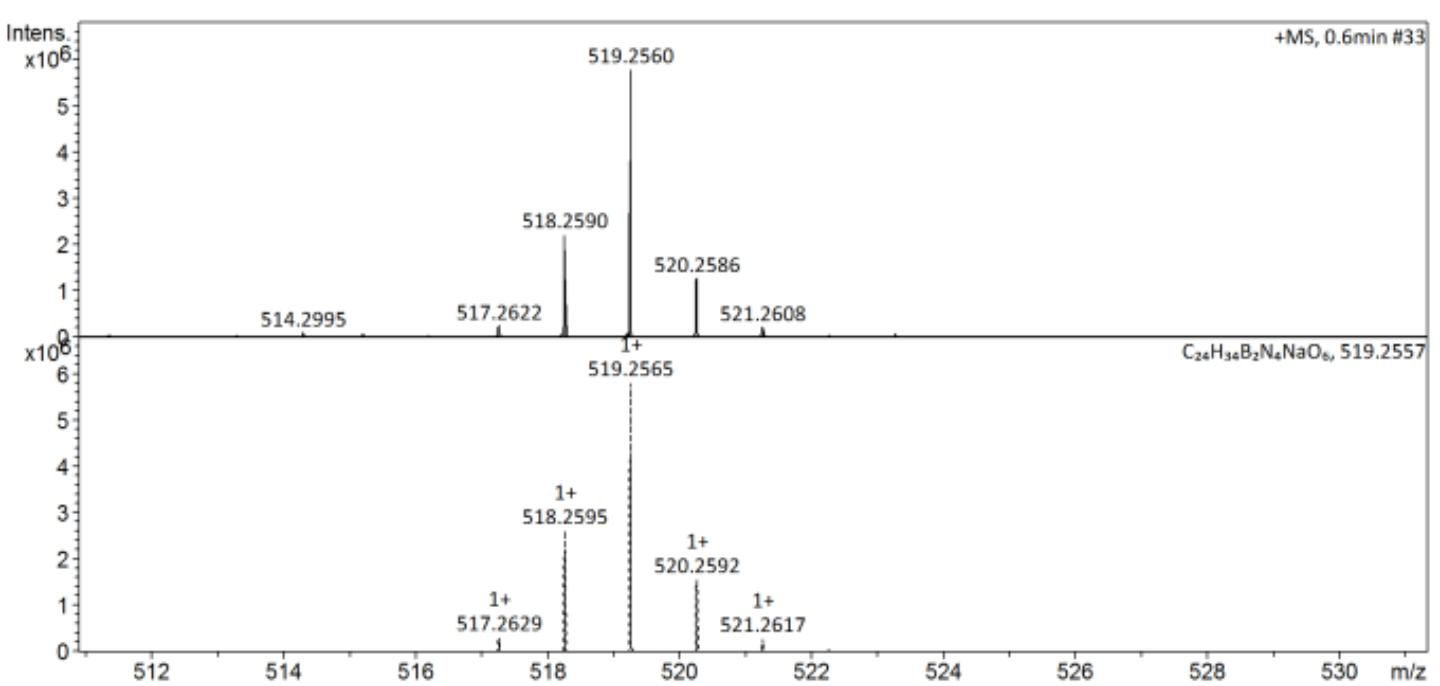

YC4-38_RB1 01_13625.

Bruker Compass DataAnalysis 4.1

\section{HR ESI-MS}

\section{AZ-2,7-DBT 6}

Figure 20. 3-(2-azidodeamino)-2,7-diboronopinacol tryptophan methyl ester (AZ-2,7-DBT) 6 
YC4-40

$170513 \mathrm{H}$

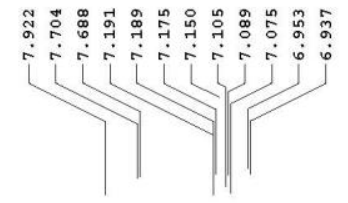

Solvent: c6d6

Ambient temperature

operator: vminr1

File: $170513 \mathrm{H}$

INOVA-500 "Varian-NMR"

Relax. delay $1.000 \mathrm{sec}$

Pulse 45.0 degree

Acq. time $2.046 \mathrm{sec}$

Wiath $7509.6 \mathrm{Bz}$

OBSERVE H1, 499.9557603 MH

OBSERVE H1, 499

DAIA PROCBSSING

Resol. enhancement $-0.0 \mathrm{H}$

Total time $1 \mathrm{~min}, 44 \mathrm{sec}$

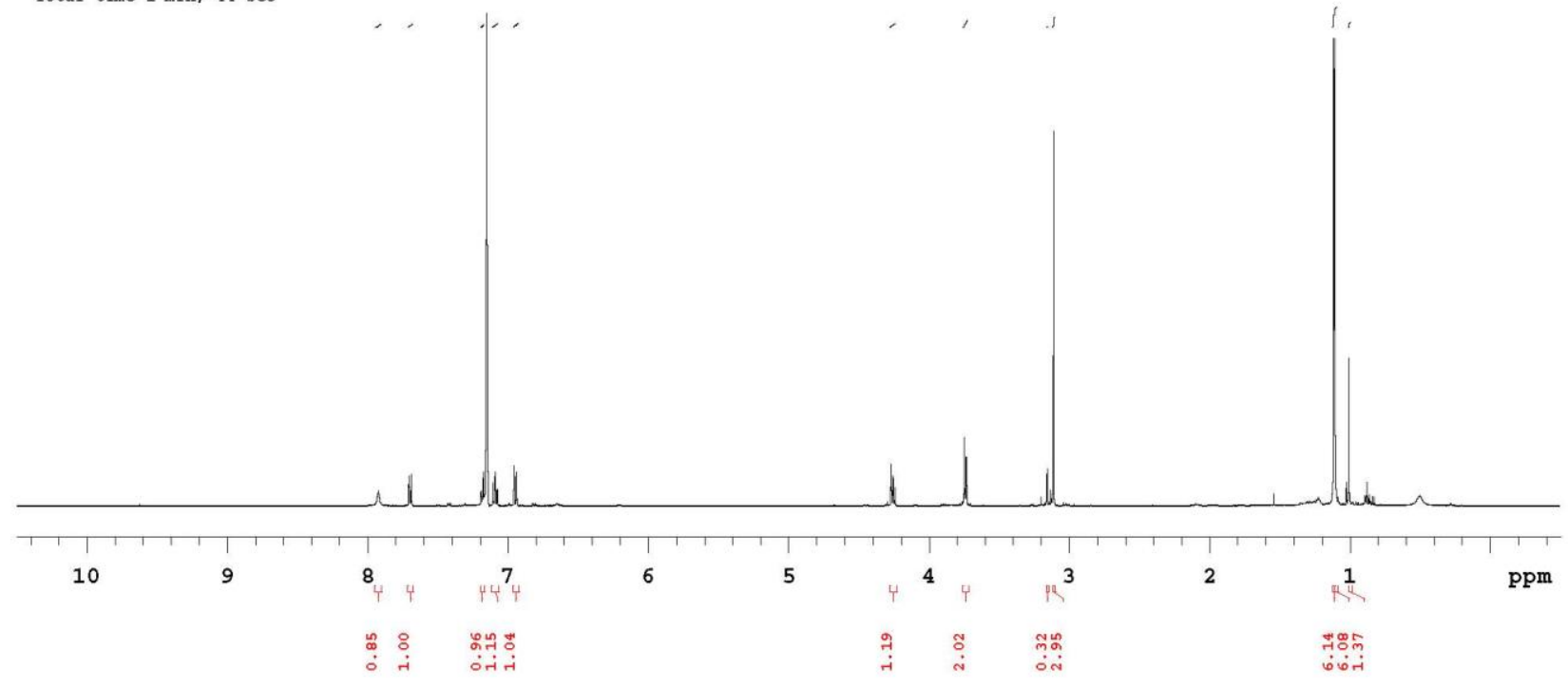

${ }^{1} \mathrm{H}-\mathrm{NMR}$ in $\mathrm{CDCl}_{3}$<smiles>CC(=O)C(Cc1c(B2OC(C)(C)C(C)(C)O2)[nH]c2ccccc12)C(C)=O</smiles>

2-BAT 18 
YC4-40

170513 DEPT

Solvent: c6d6

Ambient temperatur

Operator: vnmr1
INOVA-500 "Varian-NMR"

Relax. delay $1.000 \mathrm{sec}$

Pulse 90.0 degrees

Acq. time $1.001 \mathrm{sec}$

width $30165.9 \mathrm{~Hz}$

5000 repetitions

OBSERVE C13, 125.71

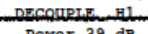

on during acquisitio

off during delay

DaTh

Iine broadening

FT size 65536

列
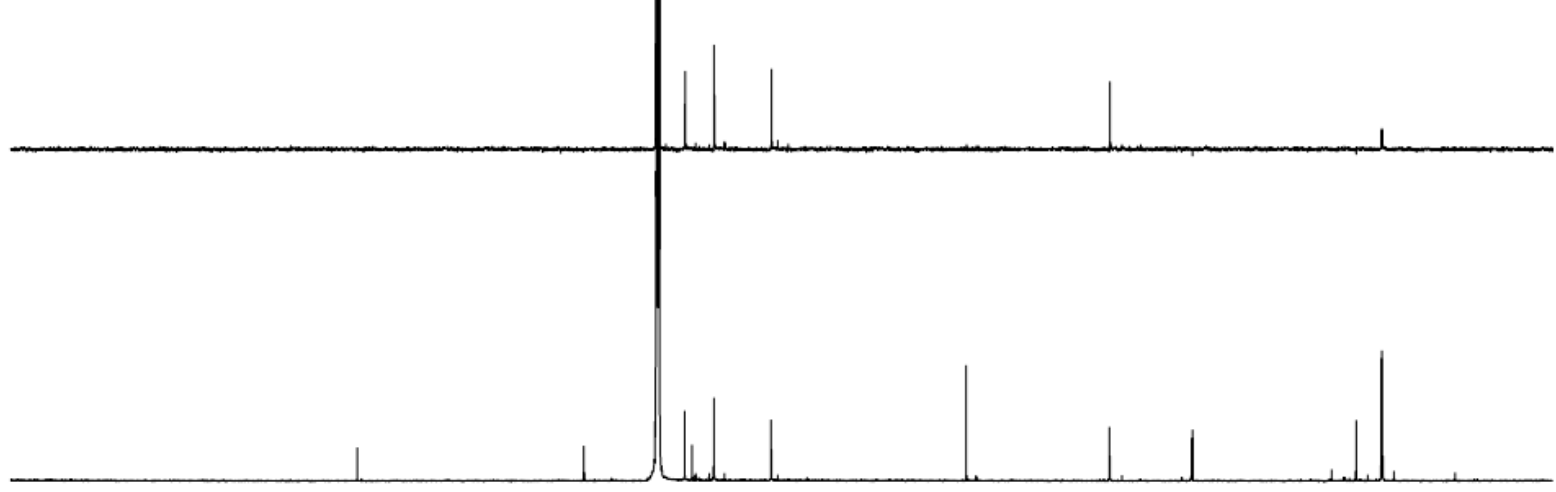

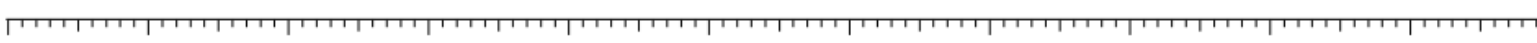

200

180

160

140

120

100

60

40

20

2-BAT 18 


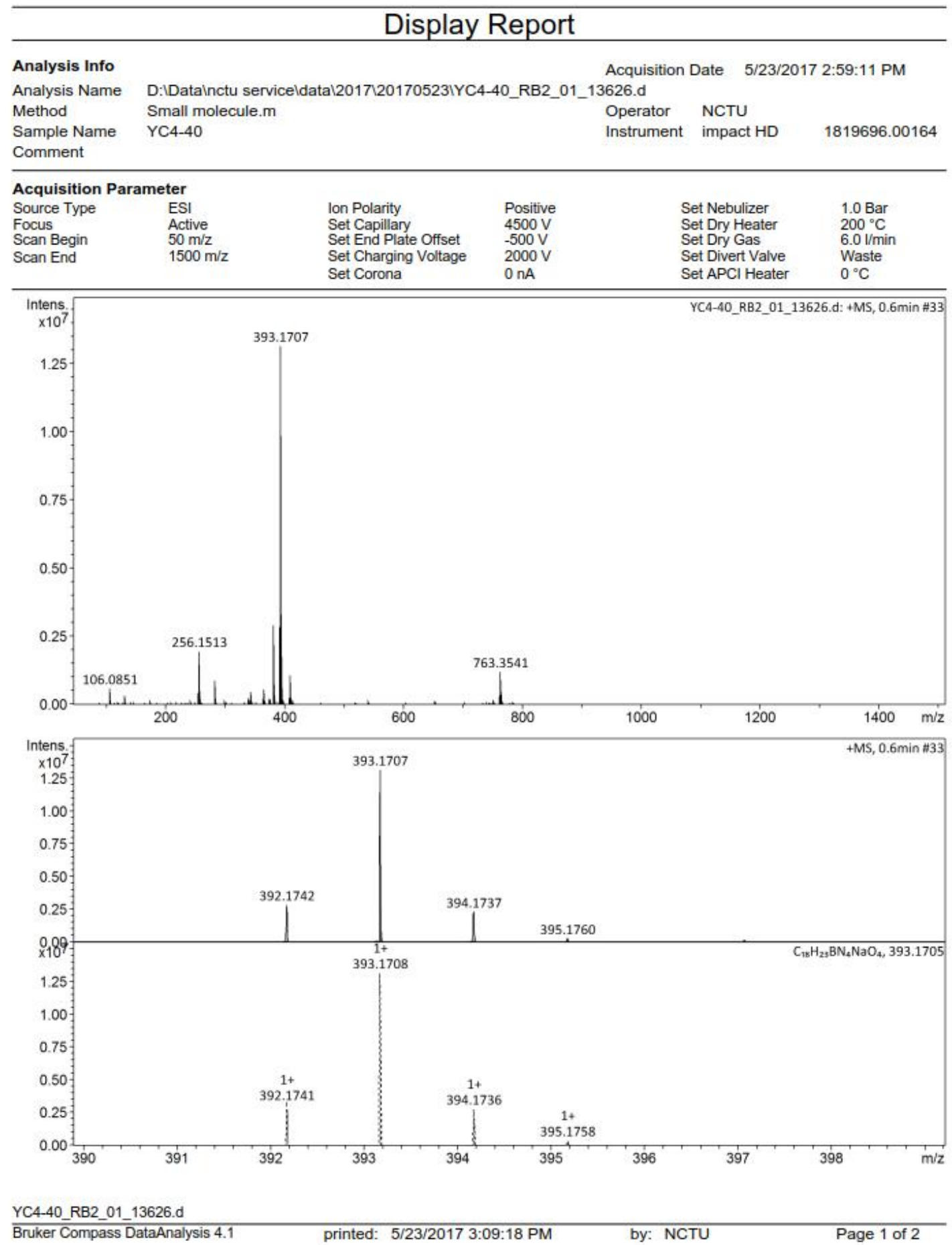

\section{HR ESI-MS}

\section{2-BAT 18}

Figure 21. 3-(2-azidodeamino)-2-boronopinacol tryptophan methyl ester (2-BAT) 18 


\section{Biological data}
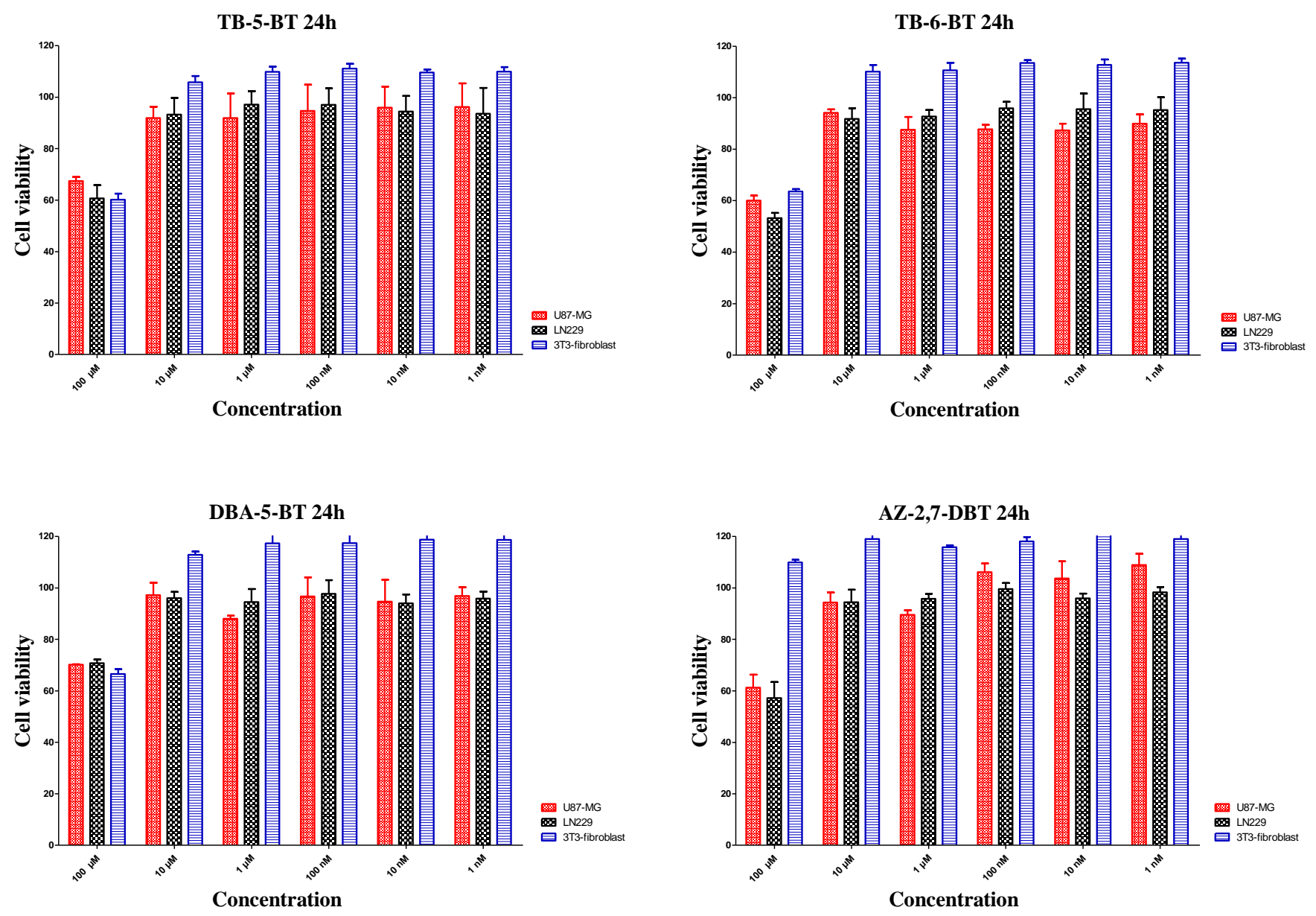

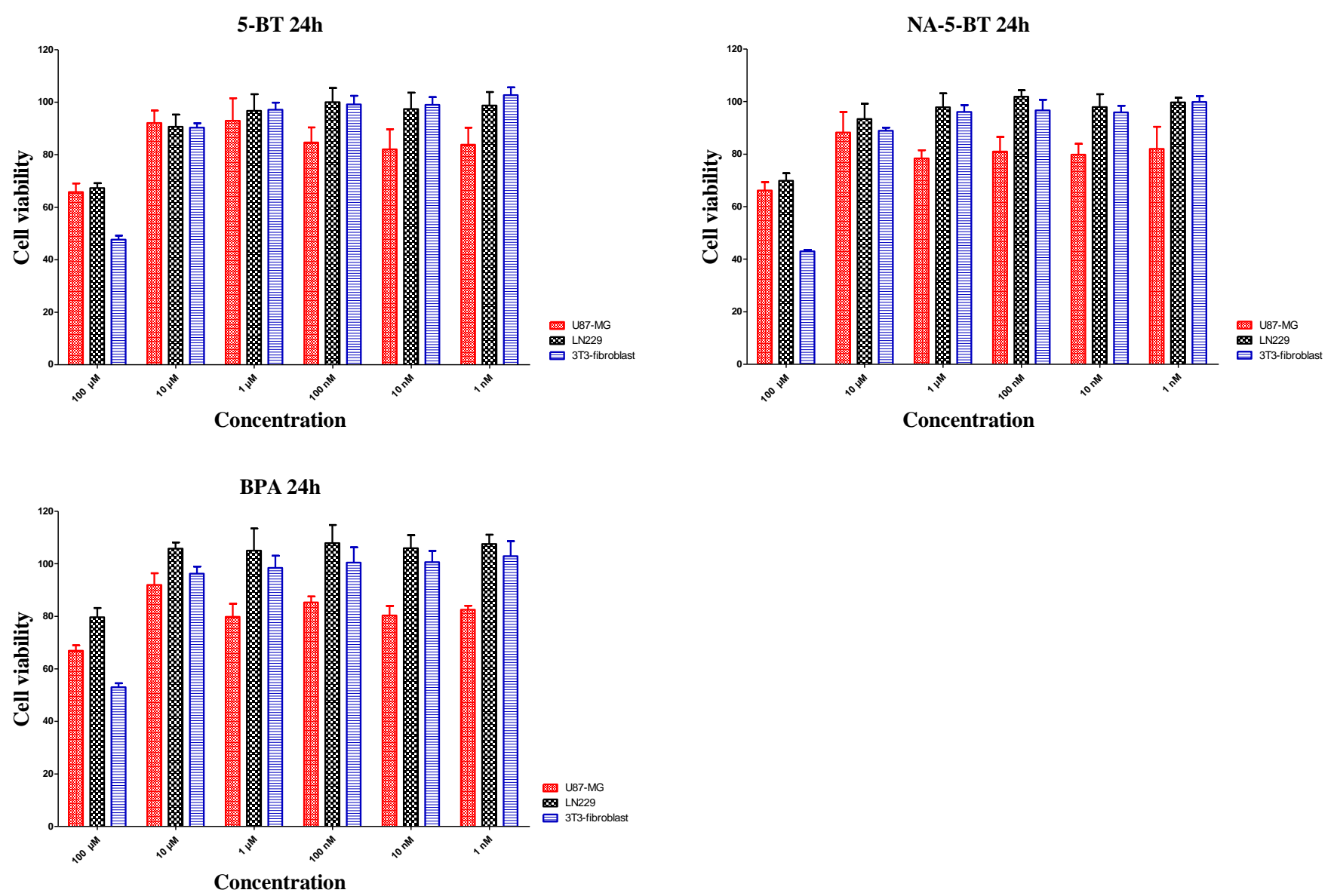

Figure 22. Cytotoxicities of boronopincaol tryptophan derivatives in various concentrations ranging from 1 $\mathrm{nM}$ to $100 \mathrm{uM}$

\section{References}

1. Giglio, B. C.; Fei, H. Y.; Wang, M. Z.; Wang, H.; He, L.; Feng, H. J.; Wu, Z. H.; Lu, H. J.; Li, Z. B., Synthesis of 5- F-18 Fluoro-alpha-methyl Tryptophan: New Trp Based PET Agents. Theranostics 2017, 7 (6), 1524-1530.

2. Kadayifcioglu, N.; Acar, H. Y., Synthesis of poly(2-acetamidoacrylic acid) and its PEGMA block copolymer via ATRP in water. European Polymer Journal 2013, 49 (10), 3366-3376.

3. Dedeoglu, B.; Ugur, I.; Degirmenci, I.; Aviyente, V.; Barcin, B.; Cayli, G.; Acar, H. Y., First RAFT polymerization of captodative 2-acetamidoacrylic acid (AAA) monomer: An experimental and theoretical study. Polymer 2013, 54 (19), 5122-5132.

4. Rothstein, E., Experiments in the Synthesis of Derivatives of Alpha-Aminoacrylic Acid from Serine and N-Substituted Serines. Journal of the Chemical Society 1949, (Aug), 1968-1972.

5. Simons, C.; Hanefeld, U.; Arends, I. W. C. E.; Sheldon, R. A.; Maschmeyer, T., Noncovalent anchoring of asymmetric hydrogenation catalysts on a new mesoporous aluminosilicate: Application and solvent effects. Chemistry-a European Journal 2004, 10 (22), 5829-5835.

6. Gladiali, S.; Pinna, L., Asymmetric Hydroformylation of N-Acyl 1-Aminoacrylic Acid-Derivatives by Rhodium/Chiral Diphosphine Catalysts. Tetrahedron-Asymmetry 1991, 2 (7), 623-632. 
7. Bartolucci, S.; Bartoccini, F.; Righi, M.; Piersanti, G., Direct, Regioselective, and Chemoselective Preparation of Novel Boronated Tryptophans by Friedel-Crafts Alkylation. Organic Letters 2012, 14 (2), 600 603.

8. Billingsley, K. L.; Barder, T. E.; Buchwald, S. L., Palladium-catalyzed borylation of aryl chlorides: Scope, applications, and computational studies. Angewandte Chemie-International Edition 2007, 46 (28), 5359-5363.

9. Tomon Moriya, K. H., A Facile Synthesis of 6-Chloro-D-tryptophan. Bulletin Of the chemical society of japan 1975, 48, 2217-2218.

10. Li, L.; Hu, W. M.; Jia, Y. X., Synthetic studies of cyclic peptides stephanotic acid methyl ester, celogentin C, and moroidin. Tetrahedron 2014, 70 (42), 7753-7762.

11. Alper, P. B.; Hung, S. C.; Wong, C. H., Metal catalyzed diazo transfer for the synthesis of azides from amines. Tetrahedron Letters 1996, 37 (34), 6029-6032.

12. Bencivenni, G.; Lanza, T.; Leardini, R.; Minozzi, M.; Nanni, D.; Spagnolo, P.; Zanardi, G., Iminyl radicals from alpha-azido o-iodoanilides via 1,5-H transfer reactions of aryl radicals: New transformation of alpha-azido acids to decarboxylated nitriles. Journal of Organic Chemistry 2008, 73 (12), 4721-4724. 